
in dynamic supramolecular systems

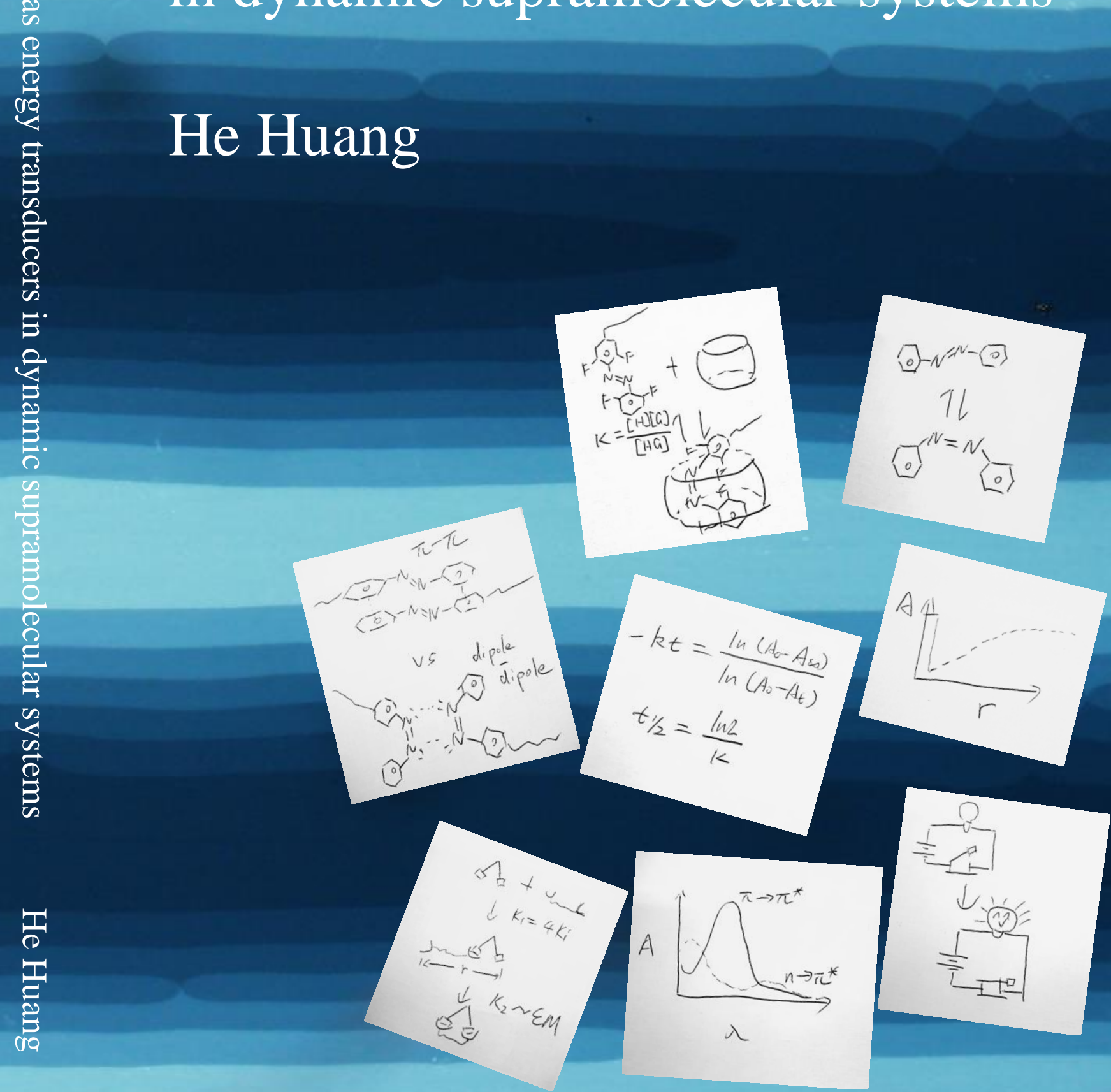




\section{Azobenzenes as energy transducers in dynamic supramolecular systems}

\section{He Huang}


Members of committee:

$\begin{array}{lll}\text { Chairman: } & \text { Prof. dr. H.H.J. ten Kate } & \text { (University of Twente) } \\ \text { Promotor: } & \text { Prof. dr. N. Katsonis } & \text { (University of Twente) } \\ \text { Co-Promotor: } & \text { Prof. dr. J.J.L.M. Cornelissen } & \text { (University of Twente) } \\ \text { Members: } & \text { Prof. dr. W.R. Browne } & \text { (University of Groningen) } \\ & \text { Dr. B. Fleury } & \text { (Sorbonne Université) } \\ & \text { Prof. dr. J.L. Herek } & \text { (University of Twente) } \\ & \text { Prof. dr. J. Huskens } & \text { (University of Twente) }\end{array}$

The research described in this thesis was performed within the group Bio-Inspired and Smart Materials, MESA+ Institute for Nanotechnology, Faculty of Science and Technology (TNW) of the University of Twente. This research was supported by the European Research Council (ERC).

\section{UNIVERSITY OF TWENTE. MESA+ \\ INSTITUTE FOR NANOTECHNOLOGY}

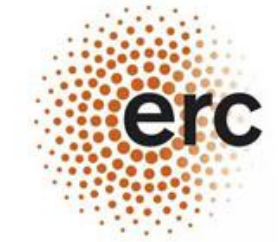

\section{Azobenzenes as energy transducers in dynamic supramolecular systems}

Copyright (C) 2018, He Huang, Enschede, The Netherlands.

All rights reserved. No part of this thesis may be reproduced or transmitted in any form, by any means, electronic or mechanical without prior written permission of the author.

ISBN

DOI:

Cover art:

Printed by:
978-90-365-4496-2

10.3990/1.9789036544962

He Huang

IPS kamp printing - the Netherlands 


\title{
Azobenzenes as energy transducers in dynamic supramolecular systems
}

\author{
DISSERTATION
}

\author{
to obtain \\ the degree of doctor at the University of Twente, \\ on the authority of rector magnificus \\ Prof. dr. T.T.M. Palstra, \\ on account of the decision of the graduation committee, \\ to be publicly defended \\ on Thursday March 8, 2018 at 14.45 h
}

by

He Huang

Born on April 20, 1987

In Liaoning, China 
This dissertation has been approved by:

Promotor: $\quad$ Prof. dr. N. Katsonis

Co-Promotor: Prof. dr. J.J.L.M. Cornelissen 
As heaven maintains vigor through movements, a gentle man should constantly strive for self-perfection.

As earth's condition is receptive devotion,

a gentle man should hold the outer world with broad mind.

----the Book of Changes

To my family 


\section{Table of Content}

Chapter 1 General introduction

Chapter 2 Bi-stable azobenzenes as photo-responsive elements 8 for supramolecular chemistry

2.1 Introduction 9

2.2 Fluorinated azobenzenes 12

2.3 Methoxy azobenzenes 24

2.4 Azoheteroarenes 28

2.5 Hydrazone and azobenzene- $\mathrm{BF}_{2}$ switches 33

2.6 Cyclic azobenzenes 36

2.7 Perspectives 39

2.8 References

Chapter 3 Long-lived supramolecular helices promoted by fluorinated photo-switches

3.1 Introduction 46

3.2 Results and discussion 48

3.3 Conclusions 55

3.4 Acknowledgements 56

3.5 Supporting information 56 
Chapter 4 Competitive inclusion of molecular photo-switches in 73 host cavities

$\begin{array}{ll}4.1 \text { Introduction } & 74\end{array}$

$\begin{array}{ll}4.2 \text { Results and discussion } & 76\end{array}$

$\begin{array}{ll}4.3 \text { Conclusions } & 84\end{array}$

$\begin{array}{ll}4.4 \text { Acknowledgements } & 84\end{array}$

$\begin{array}{ll}4.5 \text { Supporting information } & 84\end{array}$

$\begin{array}{ll}4.6 \text { References } & 95\end{array}$

Chapter 5 Host-guest complexation mediated by ortho-

fluorinated azobenzenes in supramolecular nanoparticles

5.1 Introduction $\quad 98$

$\begin{array}{ll}5.2 \text { Results and discussion } & 102\end{array}$

$\begin{array}{ll}5.3 \text { Conclusions } & 112\end{array}$

5.4 Acknowledgements 113

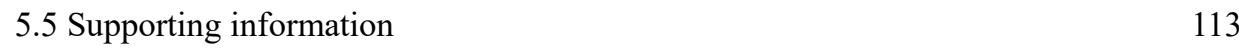

$\begin{array}{ll}\text { 5.6 References } & 121\end{array}$

Chapter 6 Light-induced self-assembly of nanoplatelets into $\quad 124$ supra-particular ribbons

6.1 Introduction 
$\begin{array}{ll}6.3 \text { Conclusions } & 136\end{array}$

$\begin{array}{ll}\text { 6.4 Acknowledgments } & 137\end{array}$

$\begin{array}{ll}\text { 6.5 Supporting information } & 137\end{array}$

$\begin{array}{ll}\text { 6.6 References } & 159\end{array}$

$\begin{array}{ll}\text { Summary } & 162\end{array}$

$\begin{array}{ll}\text { Samenvatting } & 165\end{array}$

$\begin{array}{ll}\text { Acknowledgements } & 168\end{array}$

$\begin{array}{ll}\text { About the Author } & 173\end{array}$ 


\section{Chapter 1}

General introduction 
In the past thirty years, molecular switches, motors, and machines have been introduced in dynamic systems, and involved as building blocks in a range of stimuli-responsive materials ${ }^{[1-7]}$. Among these stimuli, including redox potential, $\mathrm{pH}$, temperature or chemical stimuli, we choose to focus on light as input signal and energy source, in view of its potential towards non-invasive control of molecular photo-switches, with the possibility to achieve spatio-temporal precision ${ }^{[8-10]}$.

For the most widely used molecular photo-switches, there are two main pathways of photo-isomerization: double bond $\mathrm{E} / \mathrm{Z}$ isomerization, or ring opening/closing processes ${ }^{[7,11]}$.
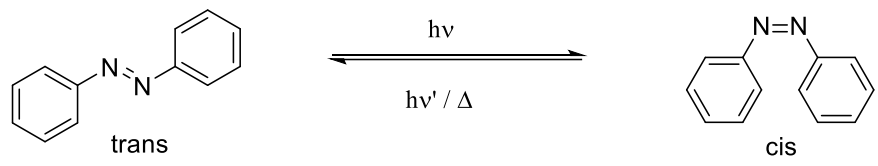

Figure 1.1. Photo-isomerization of azobenzene.

Azobenzenes are arguably the most well-known switches from the $\mathrm{E} / \mathrm{Z}$ isomerization type. An azobenzene can be switched from the planar trans-form to the bent cis- form through photo-isomerization of its $\mathrm{N}=\mathrm{N}$ double bond. Yhe reverse conversion proceeds under visible light irradiation or thermal relaxation ${ }^{[12,13]}$. The trans- azobenzene isomer is typically $10-12$ kcal mol${ }^{-1}$ more stable than its cis- form, so that the trans- isomer is the favored at equilibrium in the dark ${ }^{[14]}$ [Figure 1.1]. 


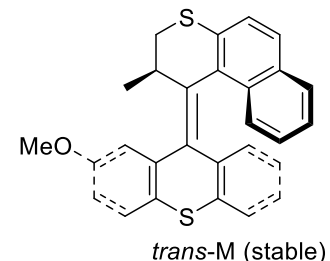<smiles>COc1ccc2c(c1)C(=C1c3ccccc3SC[C@H]1C)c1c(ccc3ccccc13)SC2</smiles>

trans-P (unstable)
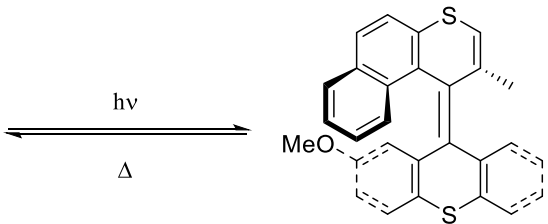

cis-P (unstable)

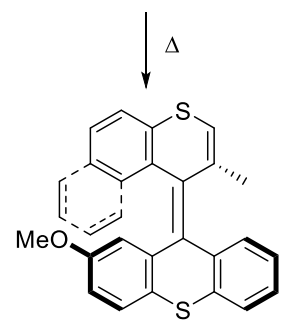

cis-M (stable)

Figure 1.2. Unidirectional rotation of a molecular motor.

Molecular motors can also undergo $\mathrm{C}=\mathrm{C}$ bond isomerization under irradiation with $\mathrm{UV}$ light, resulting in unidirectional full $360^{\circ}$ rotation through two stable $\mathrm{M}$ forms and two lessstable $\mathrm{P}$ forms. The direction of rotation is governed by the stereogenic center of the molecule ${ }^{[15,16]}[$ Figure 1.2].
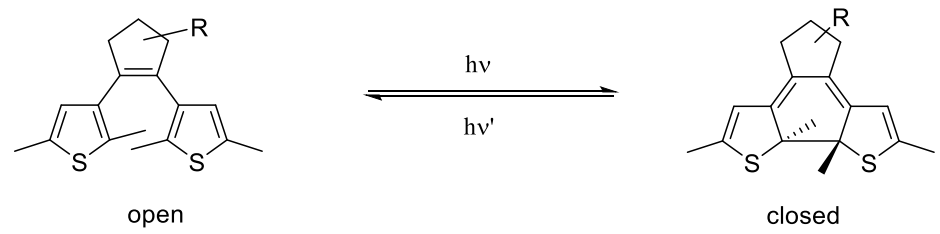

Figure 1.3. Photo-isomerization of diarylethene.

Diarylethene is a typical ring opening/closure type switch ${ }^{[17-19]}$. The $\mathrm{E} / \mathrm{Z}$ isomerization of central $\mathrm{C}=\mathrm{C}$ bond is blocked by the rigid backbone of the five-member ring. Instead, a $6 \pi$ electron pericyclic reaction with central $\mathrm{C}=\mathrm{C}$ bond and two aromatic substituents can be 
induced by UV light irradiation, resulting in photo-switching from the colorless open form to a colored closed form with a continuously $\pi$-electron conjugation system. The reverse conversion proceeds under irradiation with visible light [Figure 1.3].
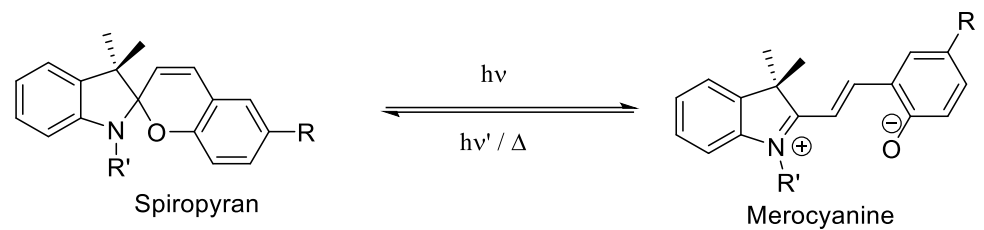

Figure 1.4. Photo-isomerization of spiropyran.

In spiropyrans, the switching between neutral spiropyran form and zwitterionic merocyanine form is also a ring-opening/closure process ${ }^{[20]}$. The photo-isomerization begins with UV-activated $\mathrm{C}_{\text {spiro }}-\mathrm{O}$ bond cleavage of the twisted spiropyran form, yields cismerocyanine ${ }^{[21-23]}$, followed by the central $\mathrm{C}-\mathrm{C}$ bond rotation ${ }^{[24]}$, results in the transmerocyanine form with a planar $\pi$-electron conjugation system ${ }^{[25,26]}$ [Figure 1.4].

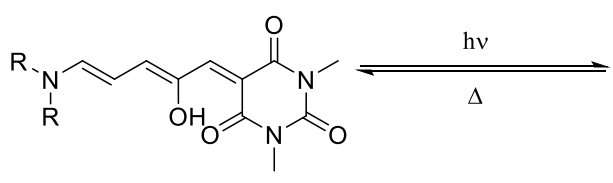

open

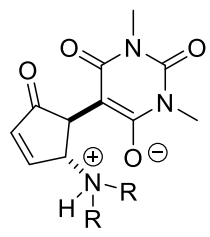

closed

Figure 1.5. Photo-isomerization of Donor-Acceptor Stenhouse Adducts.

Donor-Acceptor Stenhouse Adducts (DASAs) type switches were designed and synthesized by Hawker and co-workers. DASAs can be switched by visible light between a colored extended hydrophobic form and a colorless zwitterionic compact hydrophilic form ${ }^{[27}$, 
${ }^{28]}$. Both $\mathrm{E} / \mathrm{Z}$ isomerization and pericyclic reaction take place during the switching process. Under photo-illumination, firstly the enol $\mathrm{C}=\mathrm{C}$ double bond undergoes a $\mathrm{Z} / \mathrm{E}$ isomerization, then a thermal $4 \pi$ electrocyclization occurs on the $\pi$-electro conjugation system, yielding the ring-closed isomer ${ }^{[29,30]}$ [Figure 1.5].

In this thesis, we focus on azobenzenes as building blocks for dynamic supramolecular assemblies. As mentioned above, the trans- azobenzene is thermally more stable than its cis isomer, hence the lack of cis-state stability limits the applications of azobenzenes, because the encoded cis-state information is lost. In chapter 2, we review cis-stable azobenzenes with different molecular design strategies, and their use as building blocks in supramolecular materials for kinetic control and visible-light controlled switching.

In chapter 3, we combine ortho-fluorination of the azobenzene with axial chirality, and use the resulting molecules as chiral dopants in liquid crystals, to achieve long-lived helical organizations.

In chapter 4, we investigate the host-guest chemistry of fluorinated azobenzene with two commonly used host cavities $\beta$-cyclodextrin and cucurbit[8]uril, and in chapter 5 , we involve fluorinated azobenzene in multivalent binding systems, with the aim to achieve supramolecular nanoparticles with bi-stable assembly and dissociation states.

In chapter 6, we use azobenzene switches as photo-responsive ligands for CdSe nanoplatelets. By switching of azobenzene, the dipole-dipole interaction of cis- azobenzene induced face-to-face self-assembly of the nano-platelets into supra-particular ribbons. 


\section{References}

[1] B. L. Feringa, W. R. Browne, Molecular Switches. Second Edition. Wiley-VCH: Weinheim, 2011.

[2] W. R. Browne, B. L. Feringa, Nature Nanotech., 2006, 1, 25.

[3] E. R. Kay, D. A. Leigh, Angew. Chem. Int. Ed., 2015, 54, 10080.

[4] D. Dulic, S. J. van der Molen, T. Kudernac, H. T. Jonkman, J. J. D. de Jong, T. N. Bowden, J. van Esch, B. L. Feringa, B. J. van Wees, Phys, Rev. Lett., 2003, 91, 207402.

[5] T. Kudernac, N. Katsonis, W. R. Browne, B. L. Feringa, J. Mater. Chem., 2009, 19, 7168.

[6] J. J. D. de Jong, L. N. Lucas, R. M. Kellogg, J. H. van Esch, B. L. Feringa, Science, 2004, 304, 278.

[7] W. Szymanski, J. M. Beierle, H. A. V. Kistemaker, W. A. Velema, B. L. Feringa, Chem. Rev., 2013, 113, 6114.

[8] B. L. Feringa, Angew. Chem. Int. Ed., 2017, 56, 11060.

[9] W. R. Browne, B. L. Feringa, Nat. Nanotechnol., 2006, 1, 25.

[10] M-M. Russew, S. Hecht, Adv. Mater., 2010, 22, 3348-3360.

[11] X. Guo, J. Zhou, M. A. Siegler, A. E. Bragg, H. E. Katz, Angew. Chem. Int. Ed., 2015, 54,4782 .

[12] G. S. Hartley, Nature, 1937, 140, 281.

[13] Y. Hirshberg, Comp. Rend. Aca. Sci., 1950, 231, 903.

[14] A. A. Beharry, G. A. Woolley, Chem. Soc. Rev., 2011, 40, 4422.

[15] N. Koumura, R. W. J. Zijlstra, R. A. Van Delden, N. Harada, B. L. Feringa, Nature, 1999, $401,120$.

[16] N. Koumura, E. M. Geertsema, M. B. van Gelder, A. Meetsma, B. L. Feringa, J. Am. Chem. Soc., 2002, 124, 5037.

[17] M. Irie, Chem. Rev., 2000, 100, 1685.

[18] M. Irie, T. Fukaminato, K. Matsuda, S. Kobatake. Chem. Rev., 2014, 114, 12174.

[19] J. Zhang, Q. Zou, H. Tian, Adv. Mater., 2013, 25, 378.

[20] R. Klajn, Chem. Soc. Rev., 2014, 43, 148.

[21] S. A. Krysanov, M.V. Alfimov, Chem. Phys. Lett., 1982, 91, 77.

[22] J. T. C. Wojtyk, A. Wasey, P. M. Kazmaier, S. Hoz, E. Buncel, J. Phys. Chem. A, 2000, 104, 9046.

[23] Z. Y. Tian, W. W. Wu, W. Wan, A. D. Q. Li, J. Am. Chem. Soc., 2011, 133, 16092.

[24] B. S. Lukyanov, M. B. Lukyanova, Chem. Heterocycl. Compd., 2005, 41, 281. 
[25] J. Henzl, M. Mehlhorn, H. Gawronski, K. H. Rieder, K. Morgenstern, Angew. Chem. Int. Ed., 2006, 45, 603 .

[26] W. Fuss, C. Kosmidis, W. E. Schmid, S. A. Trushin, Angew. Chem. Int. Ed., 2004, 43, 4178.

[27] S. Helmy, F. A. Leibfarth, S. Oh, J. E. Poelma, C. J. Hawker, J. R. de Alaniz, J. Am. Chem. Soc., 2014, 136, 8169.

[28] J. R. Hemmer, S. O. Poelma, N. Treat, Z. A. Page, N. D. Dolinski, Y. J. Diaz, W. Tomlinson, K. D. Clark, J. P Hoopers, C. Hawker, J. R. de Alaniz, J. Am. Chem. Soc., 2016, $138,13960$.

[29] M. M. Lerch, S. J. Wezenberg, W. Szymanski, B. L. Feringa, J. Am. Chem. Soc., 2016, $138,6344$.

[30] M. Di Donato, M. M. Lerch, A. Lapini, A. D. Laurent, A. Iagatti, L. Bussotti, S. P. Ihrig, M. Medved, D. Jacquemin, W. Szymanski, W. J. Buma, P. Foggi, B. L. Feringa, J. Am. Chem. Soc., 2017, 139, 15596. 


\section{Chapter 2}

\section{Bi-stable azobenzenes as photo-responsive elements for supramolecular chemistry}

Azobenzenes are molecular photo-switches that are widely used as photo-responsive building blocks for dynamic and responsive materials. However, while the thermal relaxation of the cis-isomer provides spontaneous reversibility that can be useful for specific applications, in other situations, the lack of thermal stability remains a limitation in the function and performance of functional materials, and in particular, a continuously energy input is required to sustain the photo-generated state. In this chapter, we review different design strategies to develop azobenzenes with long-lived cis-isomers, and we discuss their performance as photo-responsive building blocks in supramolecular systems. 


\subsection{Introduction}

We define smart materials as materials that respond to a specific stimulus by changing a chemical or physical property and thus generating new functions, e.g. reactivity for certain chemical reactions $^{[1]}$, geometric conformation and mechanic motion ${ }^{[2,3]}$, electric properties ${ }^{[4,}$ ${ }^{5]}$ or optical properties ${ }^{[6,7]}$. Supramolecular chemistry is a useful tool to develop such materials, by building highly ordered functional architectures from small molecular building blocks through non-covalent interactions. Compared to covalent chemical bonds, with a typical energy ranging from $150 \mathrm{~kJ} \mathrm{~mol}^{-1}$ to $450 \mathrm{~kJ} \mathrm{~mol}^{-1}$ for a single bond, the non-covalent interactions are weaker, e.g. the energy for $\pi-\pi$ stacking lies between $0-50 \mathrm{~kJ} \mathrm{~mol}^{-1}$, and van der Waals interactions are associated with an energy that is below $5 \mathrm{~kJ} \mathrm{~mol}^{-1}{ }^{[8]}$. The weak interaction allows a relative low energy barrier for the material to generate responsiveness to the stimulus as well as low energy input requirements, meanwhile the side-reaction are minimized in the absence of covalent bond formation.

We focus on light as a stimulus, as a clean and precisely controllable input source that provides both command and energy to the supramolecular system, and wherein the desired function or properties are generated without chemical waste ${ }^{[9]}$. The receptor of light is a molecular photo-switch, which is defined as a molecule that undergoes reversible transformation by absorption of light between two forms, A and B, having different absorption spectra ${ }^{[10]}$. The transformation process is called photo-isomerization, or photoswitching ${ }^{[11]}$. In a supramolecular system incorporating molecular switches in its design, the input energy from light illumination is firstly converted into chemical energy, induces changes on the structure of the molecular switch itself, and consequently on its physical and 
chemical properties as well, which is followed by the interaction and alignment between the switch and its neighbor molecules, and is further transmitted to the whole system ${ }^{[12,13]}$.

In this thesis, we focus on azobenzenes as archetypal building blocks for dynamic supramolecular systems ${ }^{[14]}$, in view of their easy synthesis and functionalization ${ }^{[15]}$, their typically high extinction coefficient, and their quantum yields ${ }^{[16]}$. The simplest azobenzene 1 is shown Figure 2.1a. The distance between $\mathrm{C} 4$ and C4' in trans- form is about $9 \AA$ and in cis- form about $6 \AA$. Besides the change on geometric conformation, the molecular dipole moment also changes from $0 \mathrm{D}$ for the trans- form to $\sim 3 \mathrm{D}$ for the cis- form. The transazobenzene isomer is typically $10-12 \mathrm{kcal} \mathrm{mol}^{-1}$ more stable than the cis- form, consequently the trans- isomer is favored in the dark ${ }^{[17]}$. The reverse isomerization proceeds under irradiation with visible light, or through a thermal relaxation process. On the UV-Vis absorption spectra of the trans-form, the main absorbance band appears around $\lambda_{\max }=320$ $\mathrm{nm}$, corresponding to $\pi-\pi^{*}$ orbital transition and a weaker absorbance band appears around $\lambda_{\max }=440 \mathrm{~nm}$, corresponding $\mathrm{n}-\pi^{*}$ transition. For the cis-azobenzene, the $\mathrm{n}-\pi^{*}$ transition band normally also lies around $440 \mathrm{~nm}$, which is stronger than the trans- form, another two absorption bands are found at $\lambda_{\max }=280 \mathrm{~nm}$ and $\lambda_{\max }=250 \mathrm{~nm}^{[17]}$ [Figure 2.1b].

a)

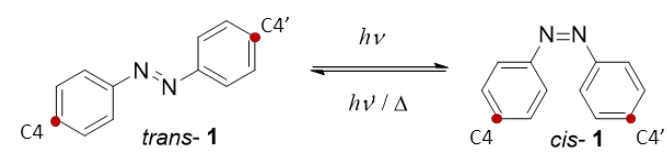

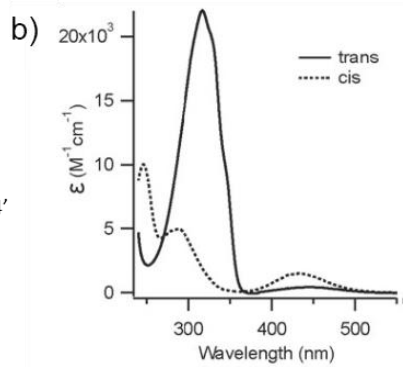

Figure 2.1. a) Structure and photo-isomerization of azobenzene 1. b) UV-Vis absorption spectrum of trans- $\mathbf{1}$ and cis- $\mathbf{1}$ in ethanol. Adapted from Ref [17] with permission of The Royal Society of Chemistry. 
Moreover, its photo-isomerization causes large changes in both the geometry and the dipole moment of the switch, thereby modifying its interaction with neighbor molecules, e.g. another azobenzene ${ }^{[18,19]}$, a host cavity ${ }^{[20]}$, surrounding bulk molecules ${ }^{[16,21-23]}$ or solvent molecules ${ }^{[24-26]}$, through non-covalent interactions and further transfer the isomerization across multiple length scales ${ }^{[13]}$.

As mentioned above, the trans- azobenzene isomer is thermally more stable than its cisform. The lack of thermal stability of the cis-form remains a limitation in some cases, typically when the cis-form switches back, the information encoded by any UV-input will be lost within several hours ${ }^{[27]}$, maintenance of the UV-encoded information requires continuous energy input ${ }^{[28,29]}$. Chemists have thus tried to develop thermally bi-stable azobenzenes. Hereafter we will show several examples of bi-stable azobenzene derivatives and how their original properties can be used in supramolecular chemistry.

\subsection{Fluorinated azobenzenes}

One strategy to design a bi-stable azobenzene, consists in functionalizing it with either electron-donating or withdrawing groups, to modify the electron density on the $c i s-\mathrm{N}=\mathrm{N}$ bond as well as the molecular orbital energy level, which could stabilize the cis-form or raise the activation energy barrier of the cis-to-trans thermal relaxation. 

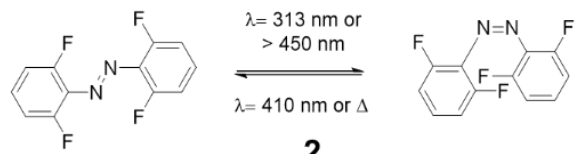

2

$\mathrm{t}_{1 / 2}=700$ days in DMSO

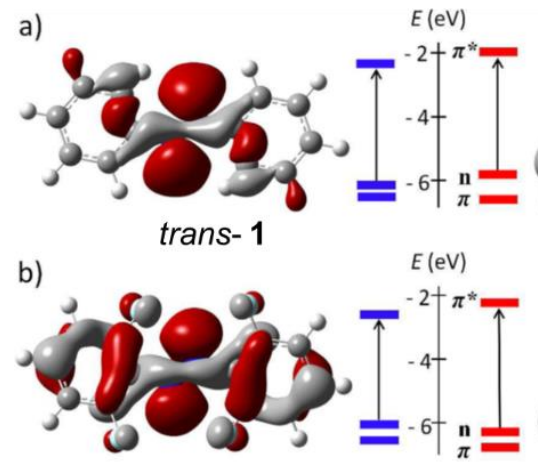

trans- 2

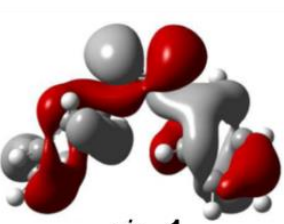

cis- 1

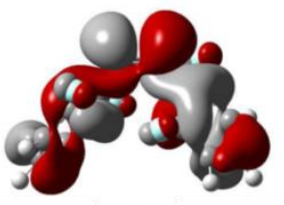

cis- 2

Figure 2.2. Molecular structure of ortho-fluorinated azobenzene 2 and energetic diagram of the $\pi, n$, and $\pi^{*}$ orbitals of a) simple azobenzene $\mathbf{1}$ and b) fluorinated derivative $\mathbf{2}$. The graph also shows a representation of the n-orbitals calculated at the B3LYP/6-31G(d) level of theory (arrows highlight $\mathrm{n} \rightarrow \pi^{*}$ transitions). Adapted with permission from Ref [30]. Copyright 2012 American Chemical Society.

Hecht, Brouwer and co-workers have shown that the cis- form of ortho-fluorinated azobenzene $(\mathrm{F}-\mathrm{Azo})$ can reach a half-live time $\mathrm{t}_{1 / 2} \sim 700$ days $^{[30]}$. In this fluorinated molecule, the electron density on the $\mathrm{N}=\mathrm{N}$ double bond was reduced by the fluorine atoms on the orthoposition, thus the $c i s$-isomer was stabilized by reducing the n-orbital energy level [Figure 2.2]. Interestingly, an earlier study suggested, that strong electron withdrawing group $-\mathrm{NO}_{2}$ on ortho- or para- position of the $\mathrm{N}=\mathrm{N}$ double bond decreases the activation energy barrier and leads to shorter half-live times for the thermal relaxation, wherein the transition state with the linear N-N-C unit was stabilized by the mesomeric effect from the electron withdrawing group as $\pi$-electron acceptors ${ }^{[27]}$. In contrast to $\pi$ acceptor $-\mathrm{NO}_{2}$ group, the fluorine atom performs inductive electro withdrawing effect instead of-mesomeric effect, 
thus the transition state of the isomerization will not be stabilized by the F-atom, resulting in a stable cis- isomer and longer half-life time.
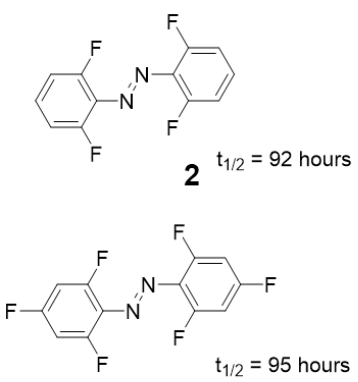

3

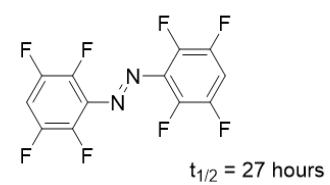

4
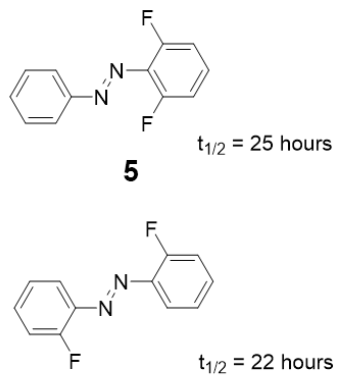

6

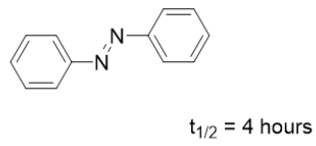

1

Figure 2.3. Fluorinated azobenzenes 2-6 and their half-life times of cis-to-trans relaxation measured in $\mathrm{MeCN}=$ at $60^{\circ} \mathrm{C}$, compared to those of non-fluorinated molecule $\mathbf{1}$.

Compounds with fluorine substitutions in various of positions and amounts were

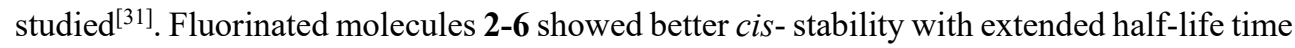
than non-modified molecule 1 [Figure 2.3]. Noticeably, the ortho-meta-octa-fluorinated azobenzene 4 is less stable than the ortho-tetra-fluorinated molecule 2, which suggested that the meta- $\sigma$-electron withdrawing group contributes negatively to the thermal stability of the cis-azobenzene. As suggested by quantum chemical calculations, the fluorine atoms on metaposition have almost no frontier orbital coefficients compare to ortho- and para-positions. On the other hand, considering the dipole moment difference between the transition state and cis- isomer, which is described as $\left(\mu^{\ddagger}\right)^{2}-(\mu(c i s))^{2}[27]$, where the $\mu^{\ddagger}$ and $\mu($ cis $)$ are the dipole moment of the transition state and cis- isomer respectively. The dipole moment difference 
for $\mathbf{2}$ is $29.8 \mathrm{D}^{2}$ and for $\mathbf{3}$ is $11.6 \mathrm{D}^{2}$, meanwhile for $\mathbf{4}$ is only $3.2 \mathrm{D}^{2}$. The difference on dipole moment influences could be also a reason for the lack of thermal stability for molecule 4 .

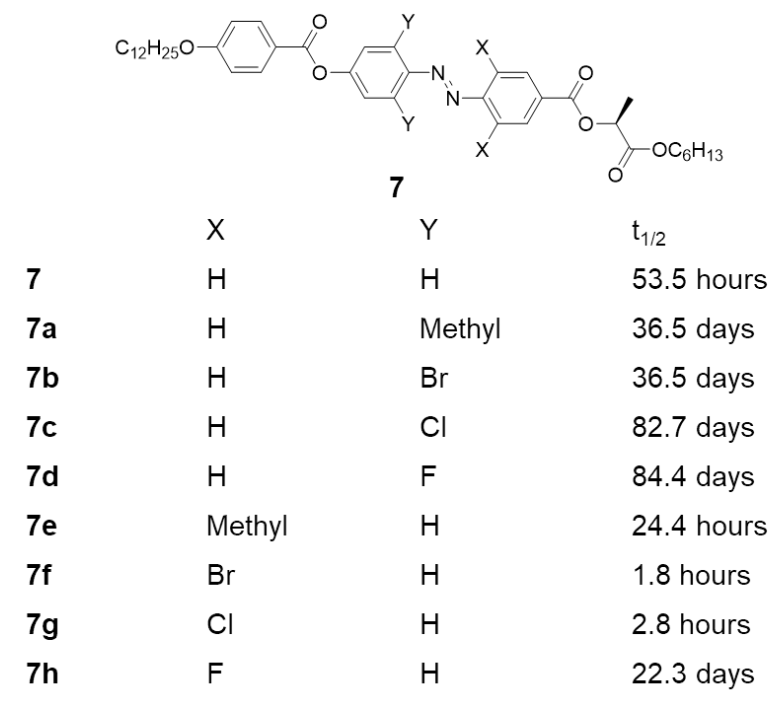

Figure 2.4. Push-pull azobenzenes 7 and $\mathbf{7 a - 7 h}$, half life time measured in $\mathrm{CDCl}_{3}$ solution at $20^{\circ} \mathrm{C}$.

Push-pull type azobenzenes typically display faster thermal relaxation rate ${ }^{[32,33]}$. Cigl et $a l$. reported a series of di-substituted azobenzenes on the para- position on one side of a pushpull type molecule ${ }^{[34]}$. Compared to the non-substituted parent molecule 7, all the molecules with substituents from "push" side (7a, $\mathbf{7 b}, \mathbf{7 c}, \mathbf{7 d})$ showed higher thermal stability. For the "pull" side functionalized switches $(\mathbf{7 e}, \mathbf{7 f}, \mathbf{7 g}, 7 \mathbf{h})$, all molecules showed decreased thermal stability than their counter part, only the fluorinated molecule $\mathbf{7 h}$ retained a moderate stability with $t_{1 / 2} \sim 22.3$ days, which is probably the result of a combined effect of positive mesomeric effect and negative inductive effect of the fluorine atoms ${ }^{[35]}$ [Figure 2.4]. 


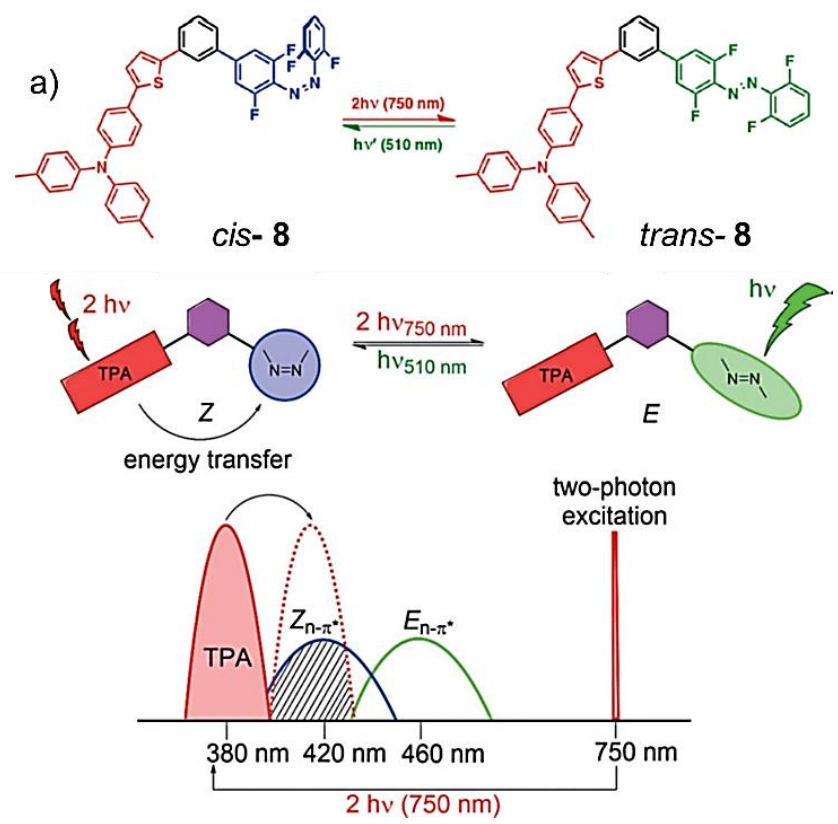

b)
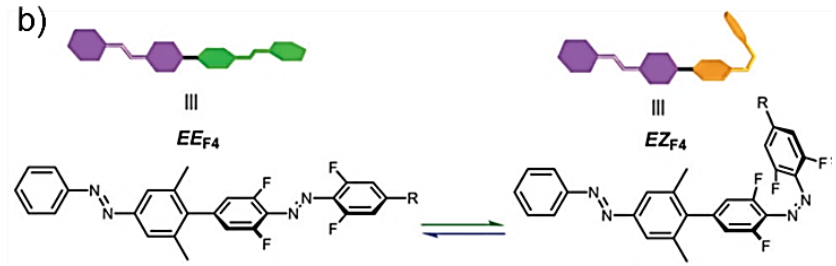

9a $\mathbf{R}=\mathbf{H}$
9b $\mathrm{R}=$ COOEt
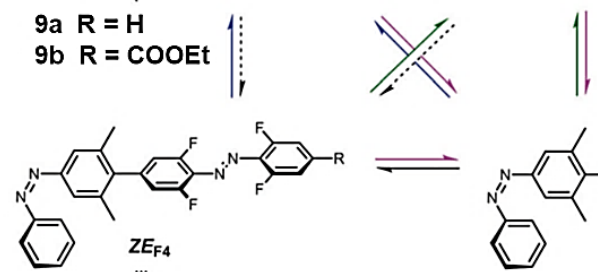

III
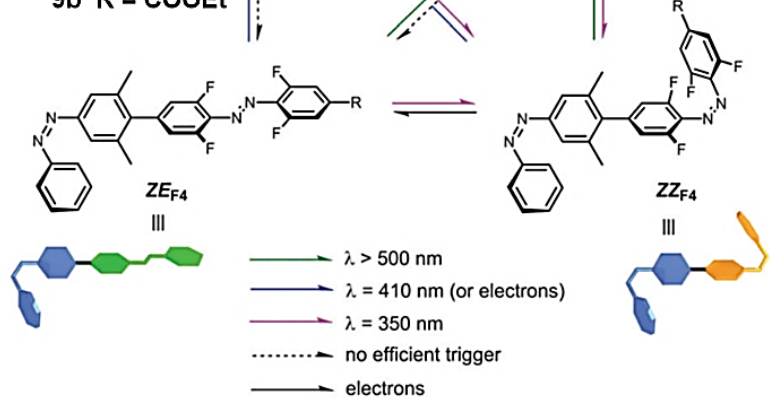

Figure 2.5. a) Structure and two-photon switching mechanism of two-photon antenna conjugated FAzo 8. Adapted with permission from Ref [36]. Copyright 2015 Wiley-VCH. b) Structure and 4-state switching process of the orthogonal molecular switches 9a and 9b. Adapted from Ref [37] with permission of The Royal Society of Chemistry. 
Fluorinated azobenzene derivatives were further introduced in a variety of molecular and supramolecular systems. In molecule 8, F-Azo was covalently bridged to a two-photon antenna group through a $\pi$-electron conjugation system, thereby to achieve a fully visible light addressable bi-stable switch ${ }^{[36]}$. The trans-to-cis photo-conversion of $\mathbf{8}$ can be triggered by green light $(\lambda=510 \mathrm{~nm})$. For the reverse conversion, the two-photon antenna could absorb two near-infrared photons $(\lambda=750 \mathrm{~nm})$ and transfer its high energetic exciton $(\lambda=380 \mathrm{~nm})$ to the cis-azobenzene moiety, resulting in cis-to-trans conversion [Figure 2.5a]. By covalently bridging fluorinated azobenzene with non-fluorinated normal azobenzene, orthogonal molecular switches $\mathbf{9 a}$ and $\mathbf{9 b}$ was synthesized ${ }^{[37]}$. The $9 \mathbf{a}$ and $\mathbf{9 b}$ could be addressed in $\mathrm{EE}_{\mathrm{F} 4}, \mathrm{EZ}_{\mathrm{F} 4}$ or $\mathrm{ZZ}_{\mathrm{F} 4}$ form by blue $(\lambda=410 \mathrm{~nm})$, green $(\lambda>500 \mathrm{~nm})$ or $\mathrm{UV}(\lambda=$ $350 \mathrm{~nm}$ ) light respectively, and for $\mathbf{9 b}$, wherein the F-Azo moiety was functionalized with an ester group, $\mathrm{ZE}_{\mathrm{F} 4}$ form could also be achieved by electro-catalytic isomerization. The two azobenzene moieties show different thermal stability, the normal azobenzene has a thermal half-life for tens of hours, and the $\mathrm{t}_{1 / 2}$ for F-Azo is up to 353 days [Figure 2.5b].

In thin crystals, azobenzenes can generate photomechanical effects ${ }^{[38-41]}$. Barrett and coworkers reported first permanent photomechanical operation on the shape of azobenzene crystal with $c i s$ - stable perhalogenated molecule $\mathbf{1 0}$ and $\mathbf{1 1}^{[42]}$. The thermal stable cis- isomers (half-life ca. 2 months) could form crystal shape changing through a solid state cis-to-trans isomerization $^{[43]}$. Under irradiation at $\lambda=457 \mathrm{~nm}$, the thin needle crystal of cis-10 or $\mathbf{1 1}$ was bended away from light source and transferred into thermal stable trans- state cyclic or zigzag shape polycrystalline [Figure 2.6]. 
a)

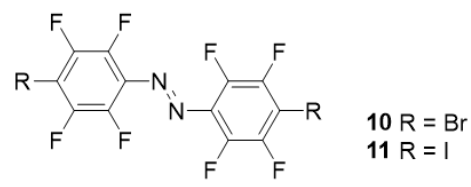

b)

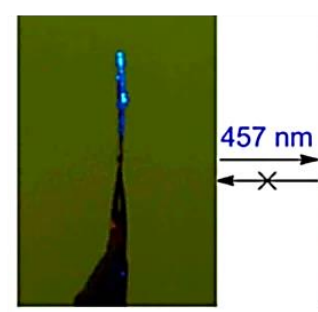

Cis-crystal

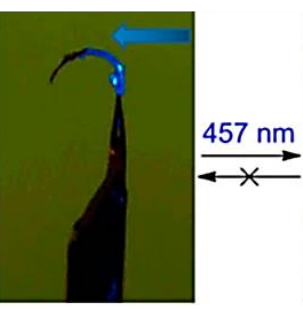

Trans-polycrystal

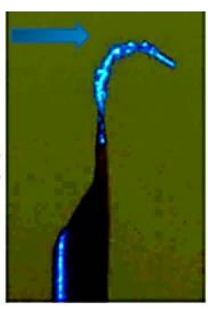

c)

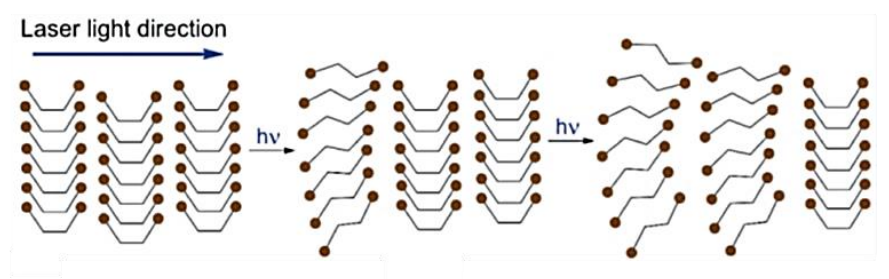

Figure 2.6. a) Molecular structure of 10 and 11. b) Irreversible bending of a thin crystal of cis-10 by $457 \mathrm{~nm}$ light, with the arrow at the top of the figures indicating the direction of irradiation. c) Tentative model of the photomechanical bending of cis-10 crystal as cis- molecules progressively isomerize into trans-10 upon light irradiation. Adapted with permission from Ref [42]. Copyright 2013 American Chemical Society.

Azobenzenes can be used as switchable dopants in liquid crystals ${ }^{[21-23]}$ : as the rod-like trans-azobenzenes are well-aligned with the surrounding nematic environments, the bentlike cis-molecules disturb this molecular alignment significantly. In liquid crystal polymer networks $^{[44-49]}$, the switching of the dopant disrupts the liquid crystal order, and thus the switching can be transferred up to the macroscopic scale, resulting in shape transformation of the material ${ }^{[50-53]}$. 
a)

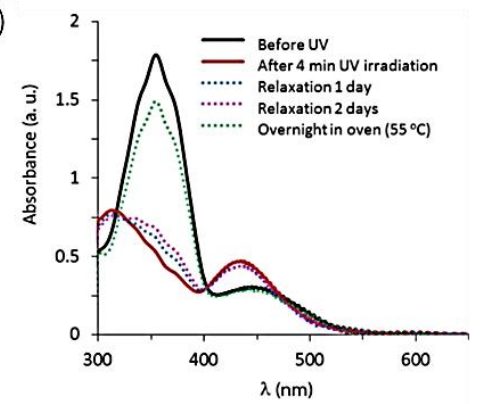

c)
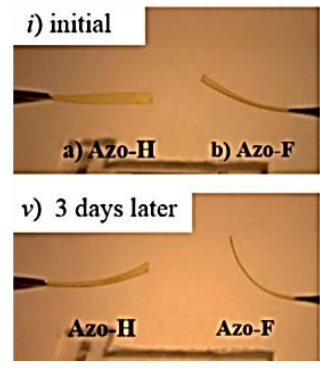
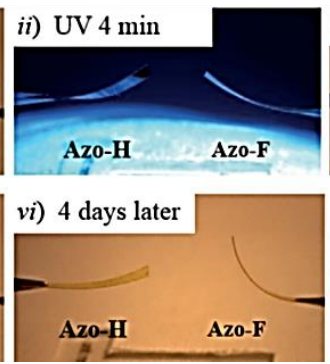

b)

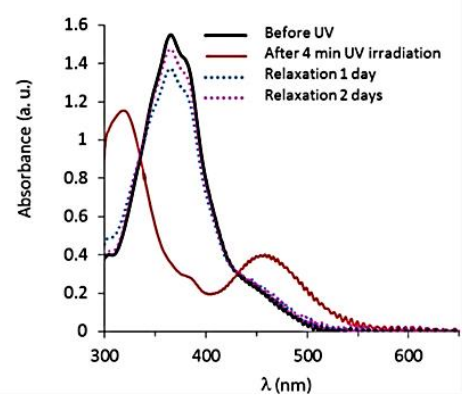

iv) $1 \mathrm{~h}$ later

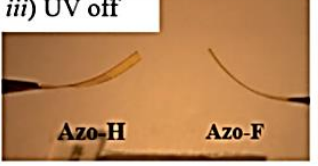

vii) 5 days later

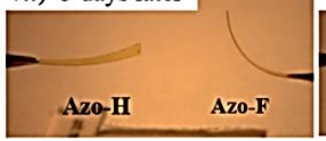

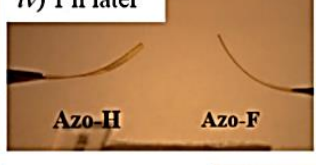

viii) 8 days later

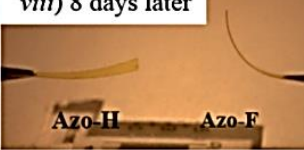

Figure 2.7. Photoswitching ( $\lambda=365 \mathrm{~nm})$ and thermal relaxation of a) normal azobenzene and $\mathrm{b})$ fluorinated azobenzene incorporated covalently into thin films of liquid crystal polymers. c) Irradiation and thermal relaxation of ribbons cut from films containing normal azobenzene and fluorinated azobenzene respectively. Adapted with permission from Ref [56]. Copyright 2016 Wiley-VCH.

For example, Katsonis and co-workers have used azobenzenes to actuate liquid crystal polymer ribbons ${ }^{[54,55]}$. Due to the lack of the cis- azobenzene thermal stability, the UV induced helices relaxed back to the initial state within several hours. Later on, a fluorinated derivative was employed as an alternative to its traditional counterpart. Due to the thermal stability of the cis-F-Azo isomer, the UV-induced shape transformation of the liquid crystal polymer was retained for at least 8 days $^{[56]}$ [Figure 2.7]. A similar strategy was applied by Schenning and co-workers but with different outcomes ${ }^{[57]}$. An identical property of the FAzo is, since the F-atom decreased the n-orbital energy of the cis- form, the $\mathrm{n}-\pi^{*}$ transition band of the cis-isomer is well-split from its trans- isomer, which allows the F-Azo to undergo 
trans-to-cis isomerization by green light and blue light for the reverse way ${ }^{[30,31]}$. Under sunlight illumination, chaotic oscillation of a liquid crystal elastomer was performed, wherein the F-azo dopants absorbed green and blue light in the same time, and photoisomerization occurred in both directions continuously. In contrary, a reference sample with non-fluorinated azobenzene dopant showed no responsibility to sunlight ${ }^{[50]}$.

Chiral azobenzenes were also used to introduced chirality and photo-responsiveness in nematic liquid crystals ${ }^{[21-23,58,59]}$. The propensity of the chiral dopants, to induce a twist in a given host is characterized by their helical twisting power (HTP), defined as HTP = $(p \times c \times e e)^{-1}$, where $p$ is the pitch and corresponds to a full $360^{\circ}$ rotation of the molecules along the helical axis, $c$ is the concentration of the dopant in $\mathrm{wt} \%$ and $e e$ is the enantiomeric excess of the dopant. Commonly, the azobenzene with axial chirality promises both higher HTP value in the ground state as well as a large variation through photo-isomerization ${ }^{[60-62]}$. As discussed in details in Chapter 3, we have designed and synthesized a new class of chiral azobenzene 12, which combined two strategies: axial chirality and fluorination on orthoposition $^{[63]}$. When used as dynamic dopant in liquid crystals, this molecule could provide sufficient HTP value and variation under photo-irradiation, while the thermal stability of the photo-induced cholesteric helix was significantly increased compare to the liquid crystal doped with its non-fluorinated counterpart 13 ${ }^{[60,61]}$ [Figure 2.8]. 
<smiles>[R]Oc1cc([X])c(N=Nc2ccc3ccccc3c2-c2c(/N=N/c3c([X])cc(O[R])cc3[X])ccc3ccccc23)c([X])c1</smiles>

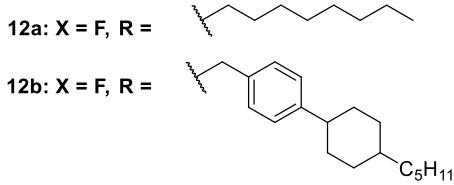

13a: $X=H, R=$ 13b: $X=H, R=$

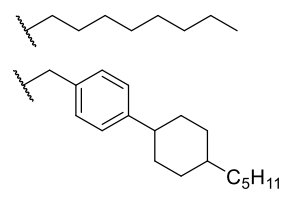

Dopant
$\begin{aligned} & 12 a \\ & 12 b \\ & 13 a \\ & 13 b\end{aligned}$

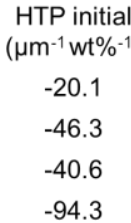

HTP at PSS $\left(\mu \mathrm{m}^{-1} \mathrm{wt} \%^{-1}\right)$

$-8.1$

$-19.0$

$-2.7$

$-23.4$

Figure 2.8. Molecular structure and photo chemical performance of chiral azobenzenes $\mathbf{1 2}$ and $\mathbf{1 3}$ used as dopants in the nematic liquid crystal E7. All values are recorded at $22{ }^{\circ} \mathrm{C}$.

Cholesteric liquid crystals are broadly employed as optical display devices ${ }^{[64,65]}$ thanks to their selective light reflection according to Bragg's law ${ }^{[66]}$. Various dynamic chiral dopants were synthesized to provide a wide range tunable reflection, that covers the full visible range ${ }^{[59-61]}$. A main limitation remained for these systems is that, the optically addressed images have to be one visible color to serve as background, wherein a certain color close to the background is not feasible. Thus, a cholesteric liquid crystal display system is required with extended tuning period up to near-infrared region and covering full visible spectrum at the same time. Qin et al. designed molecule 14, which contains two normal azobenzene units connected to an axially chiral center and two non-chiral F-Azo units ${ }^{[67]}$. This molecule can be addressed in three different states as (trans, trans, trans, trans), (cis, trans, trans, cis) and (cis, cis, cis, cis) with irradiation of blue $(\lambda=470 \mathrm{~nm})$, green $(\lambda=530 \mathrm{~nm})$ and UV light $(\lambda$ $=365 \mathrm{~nm}$ ) respectively. Noteworthy that the green light at $530 \mathrm{~nm}$ could trigger the cis-to- 
trans isomerization of the azobenzene part and trans-to-cis isomerization of the F-Azo part in parallel. When used as dopant in the nematic liquid crystal host E7, the resulting cholesteric liquid crystal could perform full-visible range selective reflection including three primary color under $470 \mathrm{~nm}$ and $530 \mathrm{~nm}$ photo irradiation. On the other hand, under $530 \mathrm{~nm}$ and $365 \mathrm{~nm}$ photo-irradiation, near-infrared region up to $1430 \mathrm{~nm}$ reflection could also be performed by the same sample. This mechanism suggested this cholesteric liquid crystal system could create images with three primary Red-Green-Blue color with a black background as a new strategy for phototunable Red-Green-Blue-Black display[Figure 2.9].

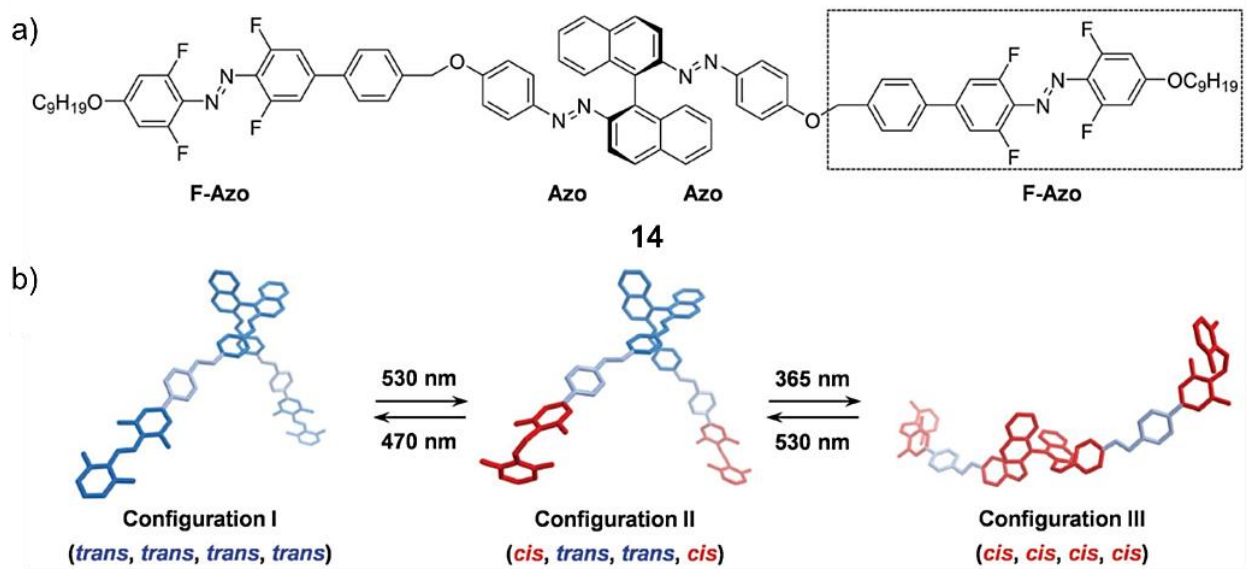

Figure 2.9. a) Chemical structure of molecule 14. b) Schematic illustration of the tristate photoisomerization of molecule 14. Trans and cis isomers are in blue and red. Hydrogen atoms and end groups $-\mathrm{OC}_{9} \mathrm{H}_{19}$ are omitted for clarity. Adapted with permission from Ref [67]. Copyright 2017 Wiley$\mathrm{VCH}$.

Host-guest chemistry is another useful tool in construction of highly ordered functional architectures through molecular recognition and specific non-covalent binding. Azobenzene is widely used as a guest molecule to form supramolecular complexes with macrocyclic host cavities $^{[20]}$. Normal azobenzenes form complexes with $\alpha$ or $\beta$-cyclodextrin $(\alpha$ or $\beta$-CD) in 
their trans- form. After photo-isomerization, the cis- isomer does not fit the CD cavity and it is thus released ${ }^{[68-76]}$. With another widely employed macrocyclic host cucurbit[8]uril $(\mathrm{CB}[8])^{[77]}$, trans- azobenzene forms a ternary complex with methyl viologen as co-guest, this complex will be dissociated under the trans-to-cis isomerization ${ }^{[78,79]}$. Normally the cisazobenzene will not form stable complex with $\mathrm{CB}[8]$, but only with additional neighbor cation as stabilizer ${ }^{[80,81]}$.
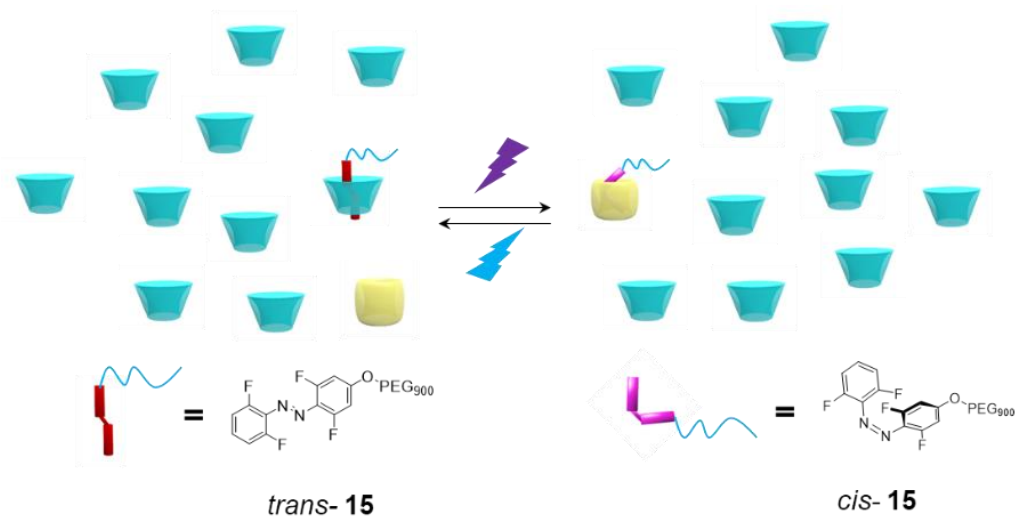

Figure 2.10. Schematic representation of the competitive host-guest inclusion of F-Azo 15 in different host cavities. Adapted from Ref [83].

Weng and co-workers firstly reported the F-Azo host-guest complexation with $\mathrm{CD}^{[82]}$. Quite different from the normal azobenzene, neither the trans- and cis- F-Azo could form host-guest complex with $\alpha$-CD, meanwhile both the trans- and cis- F-Azo could be included into the $\beta$-CD cavity, the binding affinity of cis-F-Azo is even higher than its trans- form. We studied the host-guest behaviour of an asymmetric mono-functionalized molecule $\mathbf{1 5}$ with both $\beta-\mathrm{CD}$ and $\mathrm{CB}[8]^{[83]}$. We found that i) the F-Azo with mono-functionalization will bind with $\beta$-CD from the secondary face in both trans- and cis- form, ii) the trans-F-Azo does not form any complex with $\mathrm{CB}[8]$ either with or without methyl viologen, while the cis-F-Azo 
forms a stable complex, iii) in a F-Azo, CD, CB tri-component system, it undergoes a competitive inclusion[Figure 2.10]. This will be further discussed in Chapters 4 and Chapter 5.

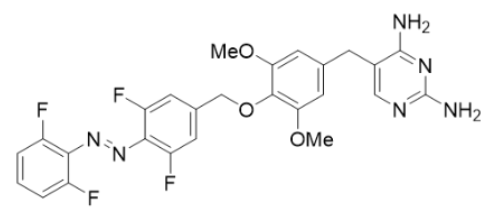

trans- 16

less active $\mathrm{MIC}_{50}=10 \mu \mathrm{M}$

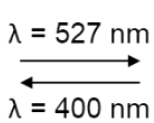

$\lambda=400 \mathrm{~nm}$

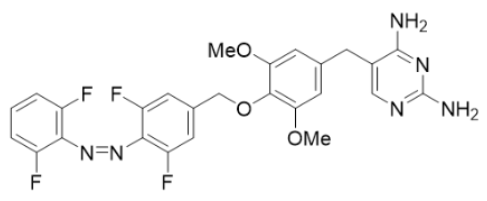

cis- 16

active

$\mathrm{MIC}_{50}=5 \mu \mathrm{M}$

Figure 2.11. Visible light driven photo-isomerization and tunable activity of antibiotic $\mathbf{1 6 .}$

Feringa and co-workers developed photo pharmacology system, whereas the antibiotic was conjugated to azobenzene to achieve photo-controlled bio-activity ${ }^{[84,85]}$. In the initial trans- state, the antibiotic showed low activity against bacteria, and got activated by UV triggered trans-to-cis isomerization. However, the cell toxicity ${ }^{[86]}$ and short tissue penetration $\operatorname{depth}^{[87,88]}$ of UV light limited the application of the azobenzene-antibiotic system. Hence, F-Azo was applied to the photo-responsive antibiotics to achieve visible light activation ${ }^{[89]}$. In compound 16, F-Azo was covalently bridged to antibiotic trimethoprim ${ }^{[90,}{ }^{91]}$. The compound 16 underwent green light $(\lambda=527 \mathrm{~nm})$ triggered trans-to-cis isomerization and performed increased activity against $E$. coli (minimum inhibitory concentration $\mathrm{MIC}_{50}$ from $10 \mu \mathrm{M}$ to $5 \mu \mathrm{M})$, and the reverse conversion was induced by violet light $(\lambda=400 \mathrm{~nm})$. When cis- 16 was kept in dark, no thermal relaxation was monitored after 24 hours, which indicated that the cis-to-trans thermal relaxation happens on much slower timescales. [Figure 2.11] 


\subsection{Methoxy azobenzenes}

Functionalizing the ortho or the para position of the $\mathrm{N}=\mathrm{N}$ group with an electron donating group usually increases the rate of cis-to-trans thermal relaxation of azobenzenes ${ }^{[92,}{ }^{93]}$. Woolley and co-workers designed and synthesized electron donating group functionalized ortho-methoxy azobenzene(m-Azo) $17^{[33,94]}$. The initial idea was to cause red shift of the absorption band and to achieve a fully visible light addressable molecular switch, which is more compatible in bio-environments. Surprisingly, besides the visible controllable switching behaviour, the m-Azo showed also an increased cis-form thermal stability. Compare to its parent molecule $\mathbf{1 8}$, cis-17 has a significantly increased thermal half-life (53 hours vs $12 \mathrm{~min}$ ) in aqueous solution [Figure 2.12]. Unlike the ortho-fluorination, which influence the electron density of $\mathrm{N}=\mathrm{N}$ bond directly through molecular orbital, the methoxy group destabilizes the n-orbital of Z-isomer through space by repulsive interactions between the $\mathrm{O}$ and $\mathrm{N}$ lone pair electrons ${ }^{[30]}$.
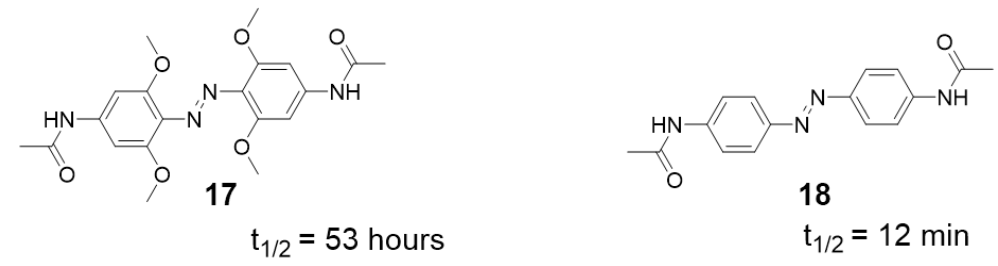

Figure 2.12. m-Azo 17 and its reference molecule 18. The thermal half-life was determined in aqueous solution. 
a)

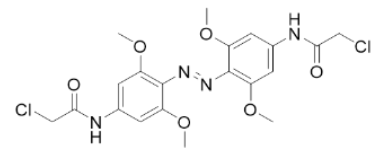

19

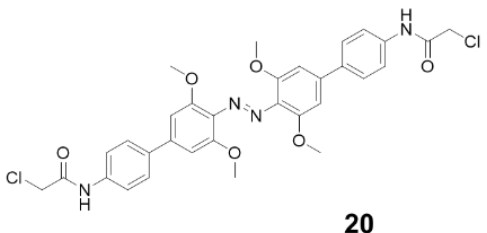

20

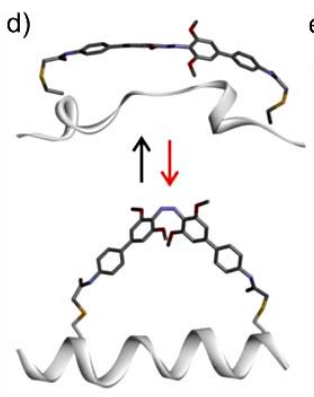

d)

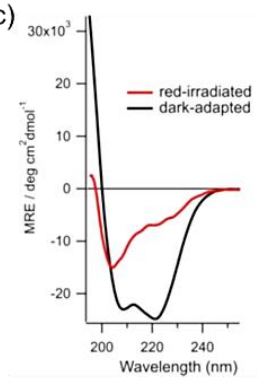

$t_{1 / 2}=6$ hours e) $30 \times 10^{3}$

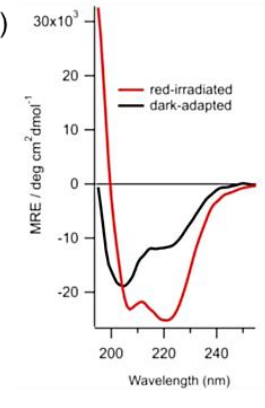

$t_{1 / 2}=45$ hours

Figure 2.13. a) Molecular structure of m-Azo 19 and 20. b) Model of the FK11 peptide cross-linked with 19 in trans- and cis- state. c) CD spectra of 19-cross-linkined FK11 indicate a red-light-driven decrease in helicity. d) Model of the FK11 peptide cross-linked with 20 in trans- and cis- state. e) CD spectra of 20-cross-linkined FK11 indicate a red-light-driven increase in helicity. Half-life time was measured at $37{ }^{\circ} \mathrm{C}$. Adapted with permission from Ref [95]. Copyright 2013 American Chemical Society.

Then the m-Azo was applied to control the conformation of bio-macromolecules. Compound 19 was cross-linking to a peptide sequence FK11 by connecting side chain -SH group of cysteines on 5- and 16- position, hence to control its helical folding behaviour by tuning the distance between these two cysteines via m-Azo photo-isomerization ${ }^{[95]}{ }^{[\text {Figure }}$ 2.13a]. According to the circular dichroism (CD) spectra, the helix content of 19-cross-linked FK11 was decreased under red-light $(\lambda=635 \mathrm{~nm})$ irradiation, which suggested that the peptide helical conformation with trans- 19 was disturbed by trans-to-cis isomerization [Figure 2.13b, 2.13c]. For the compound 20 with extended rigid backbone, the CD spectra showed an increased helicity of $\mathbf{2 0}$-cross-linked FK11 after trans-to-cis isomerization, which 
suggested that the trans-to-cis isomerization drove 20-cross-linked FK11 from coil like conformation to a highly ordered helical structure [Figure 2.13d, 2.13e]. Interestingly, the cis- 20 showed much longer thermal half-life time against cis- 19 when cross-linked to FK11 (45 hours vs 6 hours at $37^{\circ} \mathrm{C}$ ), probably the peptide helical structure also played an important role to stabilize the cis- m-Azo isomers.
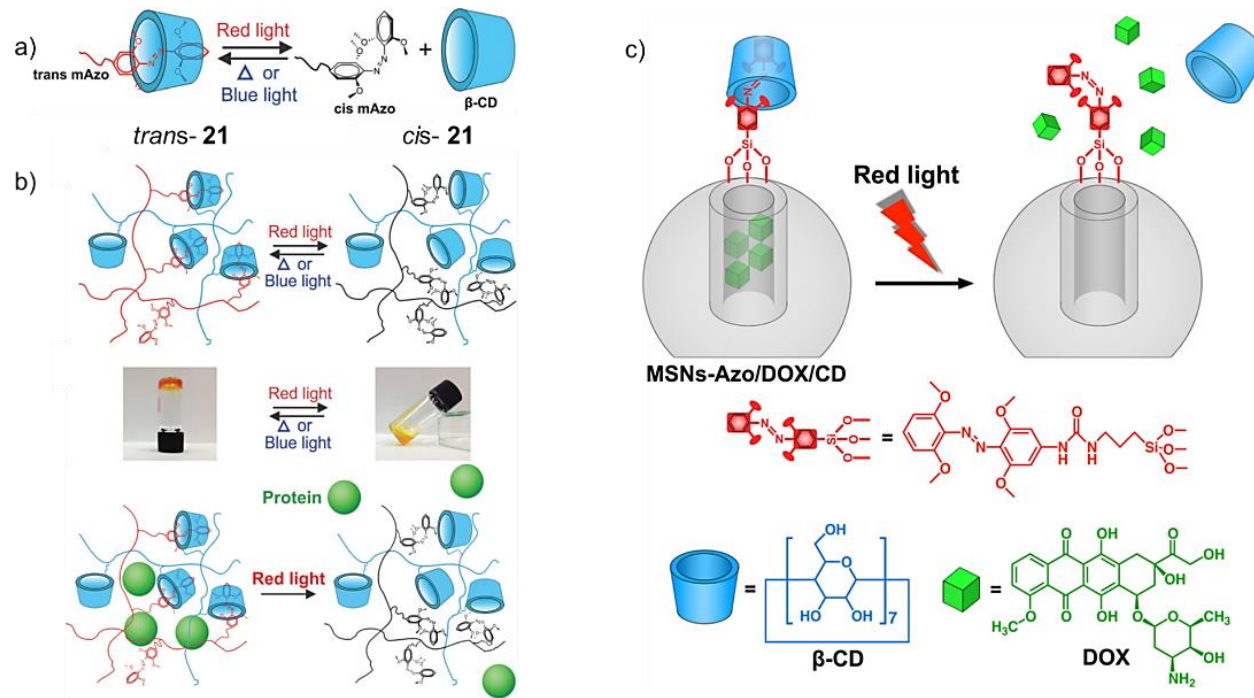

Figure 2.14. a) Schematic representation of host-guest interaction between $m-A z o$ and $\beta-C D$. b) Protein cargo release system based gel formation by m-Azo and $\beta-\mathrm{CD}$ interaction. Adapted from Ref [96]. Published by The Royal Society of Chemistry. c) Cargo release system based on host-guest pair encapped porous structures. Adapted with permission from Ref [97]. Copyright 2016 American Chemical Society.

The m-Azo was also applied to host-guest chemistry. Hindered by the methoxy group, the m-Azo cannot form any complex with $\alpha-C D$, the trans-m-Azo 21 could form host-guest complex with $\beta$-CD in its trans-form from the secondary face, under red-light illumination, the photo-isomerization causes decomplexation of the host-guest pair ${ }^{[96]}$ [Figure 2.14a]. This host-guest pair is applied to photo-responsive reversible sol-gel formation. In the trans- form 
of the m-Azo, gel was formed with host-guest pair as crosslinker, when irradiated with red light, the cis- m-Azo was released from $\beta$-CD cavity, results in solution state, protein as cargo can be released under photo-control by this sol-gel system [Fig 2.14b]. In another cargo release system, a valve function was achieved by grafting m-Azo to silica nanoparticles with mesoporous $^{[97]}$. In the trans-state, the pores were blocked by the host-guest pair with the presence of $\beta$-CD. Under red light illumination the host-guest pair was broken, thereby the pores were opened to release the cargo [Figure $\mathbf{2 . 1 4 c}$ ].

a)
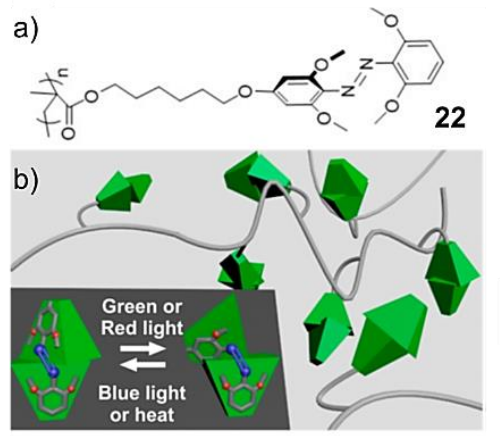

c)
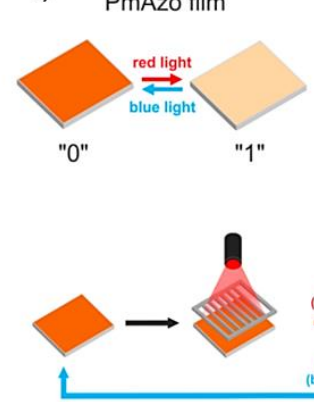

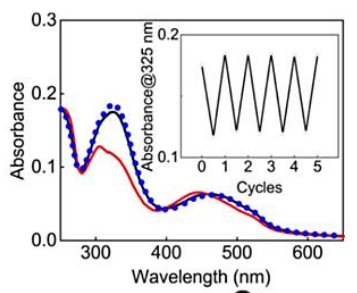

$\stackrel{\begin{array}{c}\text { Write } \\ \text { (red light) }\end{array}}{\longrightarrow}$

Figure 2.15. a) Molecular structure of polymerized m-Azo 22. b) Illustrated photo-isomerization of 22 in polymer. c) Writing and erasing function of the polymer film. Adapted with permission from Ref [98]. Copyright 2016 American Chemical Society.

Another interesting property of the m-Azo is, due to the space hinder effect of the methoxy group, the trans- m-Azo has a twisted geometry form instead of planer, hence there is no intermolecular $\pi-\pi$ stacking effects of this molecule in solid state ${ }^{[33,94]}$. A rewriteable photopatterning system was built based on this knowledge with m-Azo 22 as photoreceptor $^{[98]}$. Information could be written and stored as patterned images with red-light illumination, and reversibly erasable with blue light. Remarkably, it was found that a printed pattern was remained visible after 32 weeks stored in the dark, which is much longer than 
the half-life of a normal cis- m-Azo molecule ${ }^{[33]}$. To understand this phenomenon better, a pure cis- film was stored in the dark. Within 5 days, the $\pi-\pi^{*}$ absorption band was found as gradually increased, which indicates the thermal relaxation occurred, however the $n-\pi^{*}$ band did not overlap with the trans- form before irradiation or blue light erased sample and showed different color than the non-printed pattern. The different spectra resulted this long-term optical display and information storage [Figure 2.15].

\subsection{Azoheteroarenes}

Another modification strategy on azobenzene is to replace one or both of the phenyl rings by hetero aromatic rings, resulting in azoheteroarenes. In the past decades, the azoheteroarenes caught lot of interest, cause the cis-to-trans thermal relaxation can be controlled in a wide range from ultra-fast to very slow by varying hetero rings. In the pioneer report of molecule $\mathbf{2 3}$, one of the phenyl ring was replaced by pyrrole ring, showed normally low thermal stability with fast half-live times $(<1 \mathrm{~min})^{[99,100]}$. Velasco and co-workers developed arylazobenzothiazole $\mathbf{2 4}$, which performs extreme fast thermal relaxation at $\mu$ s level ${ }^{[101,102]}$. This kind of molecule with ultra-fast relaxation could lead to another type of application, which is opposite to our concept for this chapter: whenever the input of light source was removed, the cis- state will be transferred to trans- state immediately without any delay, which could be potentially applied into e.g. photo sensor or photo-information transfer devices. First cis- stable azoheteroarene sample is arylazoimidazole $\mathbf{2 5}^{[103]}$, which were developed by Herges and co-workers, this molecule is characterized by $t_{1 / 2} \sim 528$ hours. 
Fuchter and co-workers designed arylazopyrazoles ${ }^{[104]}$, the longest half-live time was observed from the molecule $\mathbf{2 6}$ with $\mathrm{N}=\mathrm{N}$ double bond connected to 4-position of a pyrazole ring, as about 1000 days in DMSO solutions, and also almost quantitative trans-to-cis conversion ratio. Systematic study was reported by Calbo et al. ${ }^{[105]}$, a series of azoheteroaryl photoswitches with one phenyl ring and one 5-membered hetero ring were scoped to investigate two parameters of the photo-switching process: photo-switching efficiency and cis- form thermal stability. The results suggested that cis- form conformation plays important roles on both the switching efficiency and thermal stability. However, these two requirements worked as a competition: a twisted cis- conformation is favored for more complete isomerization ratio, and for a higher thermal stability it requires a T-shape cis- state conformation, where the $c i s$-form is stabilized by $\mathrm{H}-\pi$ electron interaction. For example, the azopyrazol 26 has the longest thermal half-life among all samples for about 1000 days, with over $98 \%$ yield of trans-to-cis conversion ratio, meanwhile cis-to-trans conversion ratio is only about 70\%. In comparison, the methylated AAP (m-AAP) 27 performs a thermal halflife for only about 10 days, but the photo-switching from both directions could reach a ratio for over $98 \%$. A balance between the switching ratio and cis- form stability was achieved on the molecule 28, which could be switched almost quantitatively from trans-to-cis form (> $98 \%)$, and reverse way with green light $(\lambda=532 \mathrm{~nm})$ irradiation $(97 \%)$, half-live time was observed as 74 days at room temperature [Figure 2.16]. 

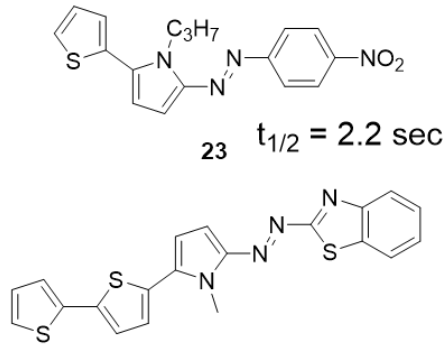

$24 t_{1 / 2}=70 \mu \mathrm{sec}$

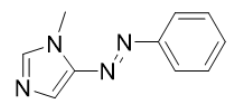

$t_{1 / 2}=528$ hours

25
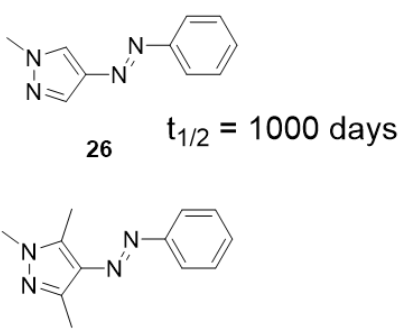

$27 \quad t_{1 / 2}=10$ days

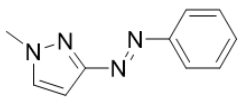

$t_{1 / 2}=74$ days

28

Figure 2.16. Azoheteroarene with various of thermal relaxation rate from ultra-fast to very slow.

Unlike the fluorinated azobenzene, which shows almost no binding selectivity to $\beta$-CD at trans- and cis- form ${ }^{[82,83]}$, the m-AAP performed similar host-guest binding behaviour to normal azobenzene, which could form host-guest complex with $\beta$-CD in its trans- form, and after photo-isomerization, the $c i s$-isomer will be released from the cavity ${ }^{[106]}$. To study the host-guest binding mechanism, m-AAP 29 and $\mathbf{3 0}$ are synthesized with different side functionalization, due to the steric effect of methyl groups, the trans-m-AAP is always combined with its phenyl ring into the secondary face of $\beta$-CD [Figure 2.17a]. With m-AAP dimer as crosslinker, the photo-responsive reversible aggregation and dispersion behaviour was achieved with cyclodextrin vesicles [Figure 2.17b] or CD functionalized gold nanoparticles [Figure 2.17c] 
a)
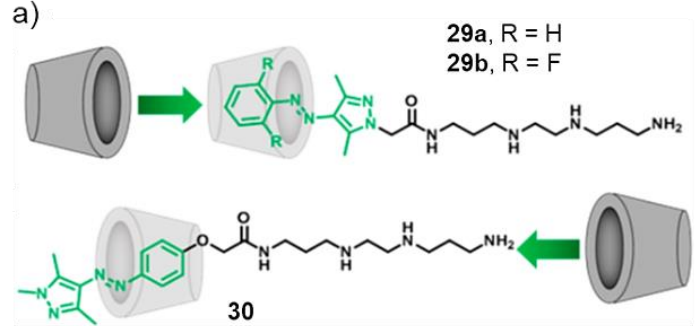

b)
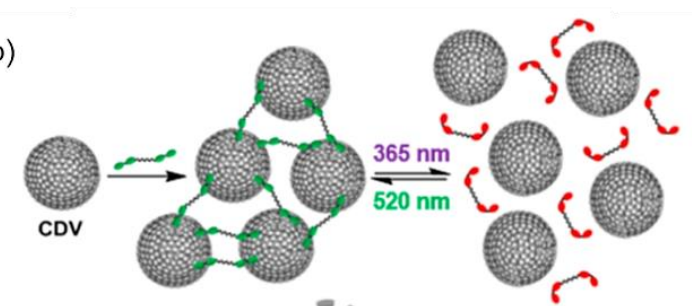

c)

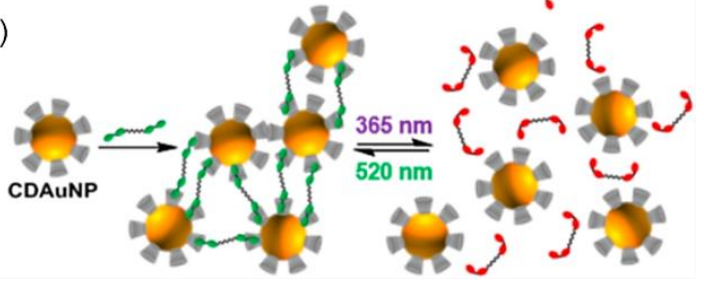

Figure 2.17. a) Predicted binding mechanism for m-AAP 29a, 29b and 30; b) Schematic representation of photo-controlled aggregation and representation on $\mathrm{CD}$ vesicles and c) $\mathrm{CD}$ functionalized gold nanoparticles. Adapted with permission from Ref [106]. Copyright 2016 American Chemical Society.

Sagebiel et al. applied this photo-controlled crosslinking strategy based on host-guest interaction between m-AAP and $\beta-C D$ to multiple length scale ${ }^{[107]}$. An ABA type Janus micro silica particle with a diameter of $6.65 \mu \mathrm{m}$ was functionalized with m-AAP containing polymers on its " $A$ " side. $\beta$-CD functionalized magnetic $\mathrm{Fe}_{3} \mathrm{O}_{4}$ nanoparticles with a diameter of $10 \mathrm{~nm}$ was employed as magnetic glue in aqueous solution. In the initial state, the Janus particles could be joined together to form worm-like oligomers by host-guest interactions between the m-AAP on the silica microparticle and $\beta-\mathrm{CD}$ on the $\mathrm{Fe}_{3} \mathrm{O}_{4}$ nanoparticles. The oligomers perform regulated motion under external magnetic field. Additionally, residuals of 
none-crosslinked Janus particles with $\mathrm{CD}-\mathrm{Fe}_{3} \mathrm{O}_{4}$ attached can also be observed, and these particles were self-rotated under the external magnetic field. When the m-AAP was switched to $c i s$-form, the host-guest pair as well as the worm-like oligomers of the silica particles were disassembled, meanwhile the magnetic responsibility on silica particles was also lost [Figure

\subsection{8].}

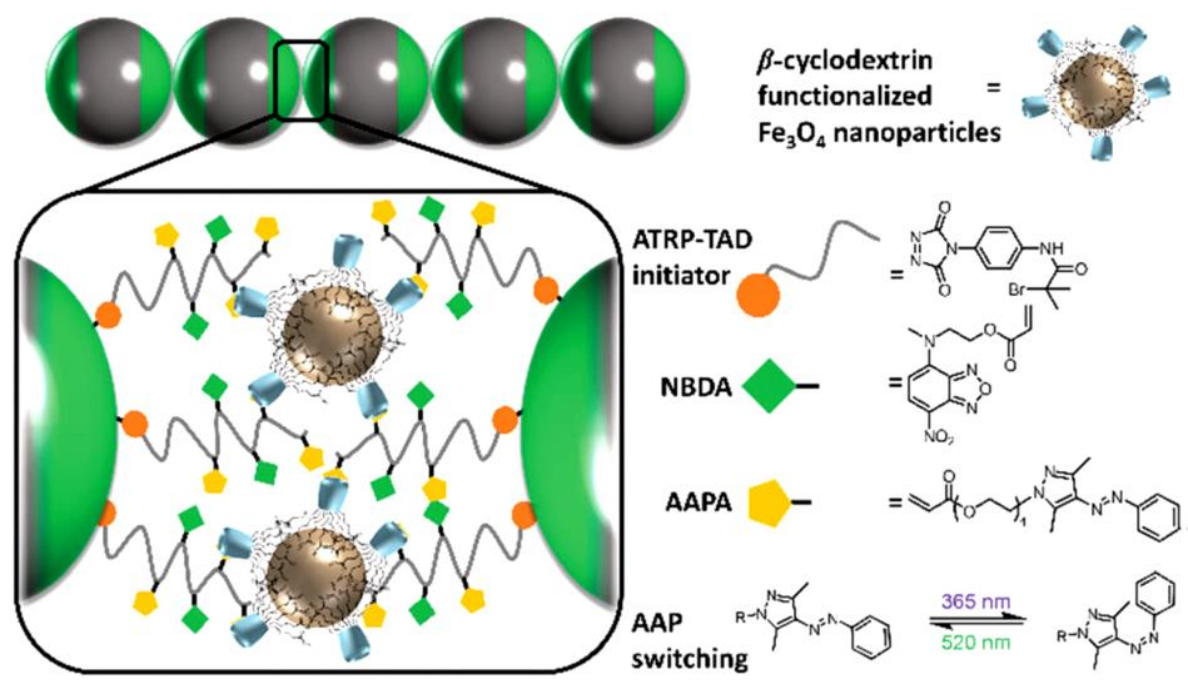

Figure 2.18. Dual responsive self-assembly of silica Janus microparticles functionalized with m-AAP containing polymers and $\beta$-CD functionalized $\mathrm{Fe}_{3} \mathrm{O}_{4}$ nanoparticles. Adapted from Ref [107] with permission of The Royal Society of Chemistry.

Another supramolecular system based on m-AAP and $\beta$-CD interactions was presented by Möller et al. ${ }^{[108]}$. Upconversion nanoparticles $\mathrm{LiYF}_{4}$, which absorbs near-infrared photons

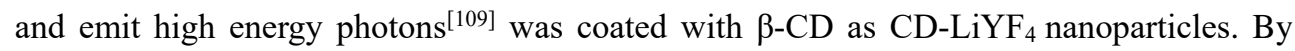
adding m-AAP into the system, host-guest pair was formed on the CD-LiYF 4 surface. When irradiate with near-infrared light $(\lambda=980 \mathrm{~nm})$, the $\mathrm{LiYF}_{4}$ emit $350 \mathrm{~nm}$ photons. The emitting photon drove the photo-isomerization of the m-AAP, led to decomplexation of the host-guest 
pair. With m-AAP dimer as crosslinker, this $\mathrm{CD}-\mathrm{LiYF}_{4}$ nanoparticles could perform lightcontrolled aggregation and dispersion due to the host-guest complexation and dissociation [Figure 2.19]. Since the irradiation condition is changed from UV light to near-infrared light, this system shows better compatibility in a biological environment.

a)

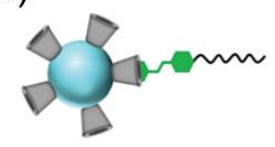

b)

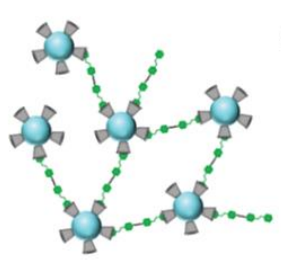

$980 \mathrm{~nm} \quad 350 \mathrm{~nm}$
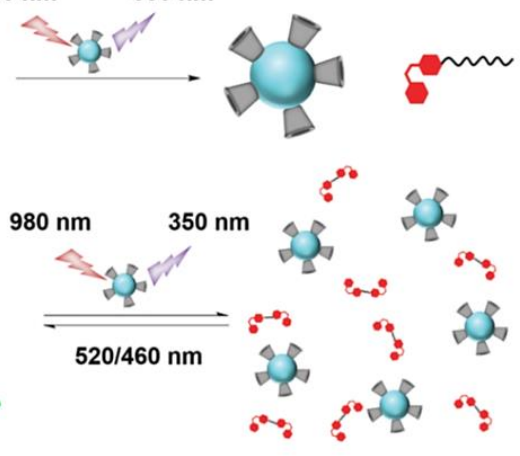

Figure 2.19. a) Host-guest interaction of $m-A A P$ in the $C D$ cavity on upconversion nanoparticle surface and release after irradiation at $\lambda=980 \mathrm{~nm}$. b) Light-controlled aggregation and dispersion with m-AAP dimer as cross-linker. Adapted from Ref [108] with permission of The Royal Society of Chemistry.

\subsection{Hydrazone and azobenzene- $\mathrm{BF}_{2}$ switches}

Courtot et al. described reversible photo-switching behaviour of a family of 1,2,3tricarbonyl-2-arylhydrazones, where the $\mathrm{N}-\mathrm{H}$ on hydrazone moiety as proton donor could form intramolecular H-bond with different acceptor carbonyl groups selectively under different wavelength light exposures ${ }^{[110-112]}$. Inspired by the tricarbonyl-arylhydrazones, Aprahamian and co-workers designed hydrazone switches, that undergo trans-/cisisomerization under $\mathrm{pH}$ control ${ }^{[113-115]}$. Later on, a light switchable azobenzene-BF2 (Azo- 
$\mathrm{BF}_{2}$ ) compound was developed ${ }^{[116]}$, which can be activated by visible light to undergo trans/cis- isomerization ${ }^{[117]}$ [Figure 2.20].

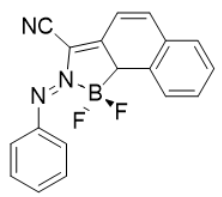

trans-31

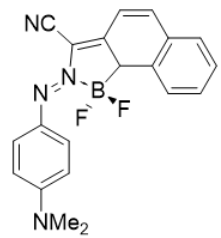

trans-32

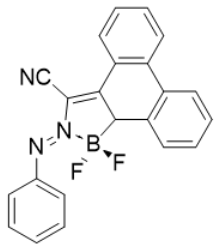

trans-33

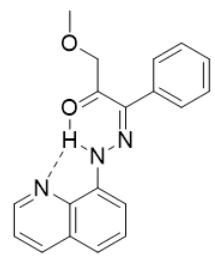

cis-34

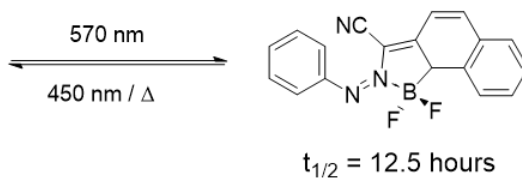

cis-31
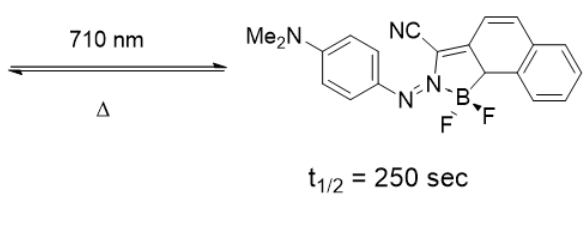

$t_{1 / 2}=250 \mathrm{sec}$

cis-32
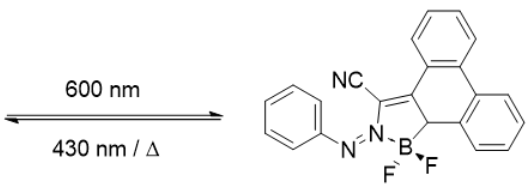

aggregation dependent thermal relaxation

cis-33
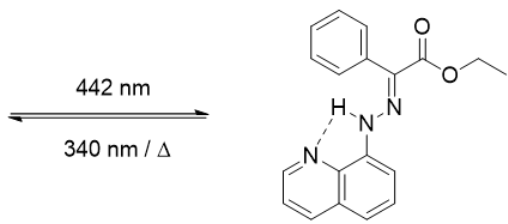

$t_{1 / 2}=2700$ years

trans-30

Figure 2.20. Azo- $\mathrm{BF}_{2}$ type switches and photo-active hydrazone switch.

To get verified switching light wavelengths, different of substituents were studied. Not surprisingly, the substituents electronic effect on the phenyl rings played an important role on the switching condition, meanwhile the thermal stability of the Azo-BF2 compounds was 
influenced $^{[118]}$. Comparing compound $\mathbf{3 1}$ and 32, the non-substituted molecule $\mathbf{3 1}$ can be switched by $\lambda=570 \mathrm{~nm}$ light from trans- form to $c i s$-form, the reverse conversion was triggered by $\lambda=450 \mathrm{~nm}$ light or trough thermal condition with a half-life time for 12.5 hours, while the electron donating group $-\mathrm{NMe}_{2}$ on the para-position increased the antibonding $\left(\pi^{*}\right)$ orbital of compound $\mathbf{3 2}$ and decreased effective bond order of the $\mathrm{N}=\mathrm{N}$ bond, resulting in lower energy barrier for isomerization from both directions, performed as a significantly redshifted switching wavelength $(\lambda=710 \mathrm{~nm})$ and a much faster thermal relaxation with $\mathrm{t}_{1 / 2}=$ $250 \sec ^{[119,120]}$.

The cis- to-trans isomerization of azobenzene is typically known as a first-order reaction, i.e. the rate of thermal relaxation is independent from concentration. Interestingly, in a recent study, the quinolinyl ring of the Azo- $\mathrm{BF}_{2}$ switch was expanded into a phenanthridinyl one, yielding molecule $\mathbf{3 3}^{[121]}$. The half-life time of cis- $\mathbf{3 3}$ at low concentration $\left(10^{-5} \mathrm{M}\right)$ was hundreds of seconds, when concentration was increased to $10^{-4} \mathrm{M}$, the half-life increased to 27 hours, and further at $10^{-3} \mathrm{M}$ solution, the half-life increased to 195 hours. Through ${ }^{1} \mathrm{H}$ NMR measurement, dynamic light scattering analysis and X-ray crystallographic analysis, it was found that the Azo-BF2 compound aggregates in solution due to the increased $\pi-\pi$ stacking effect through the phenanthridinyl core, wherein the switching kinetic was influenced. In diluted solutions, the compounds behave like free molecules, when the concentration increased, the molecular degree of freedom was limited by the aggregations, resulting in higher the concentration, slower the thermal relaxation.

Qian et al. reported a photo-responsive hydrazone molecule $\mathbf{3 4}^{[122]}$. Due to the cis- state intramolecular H-bond and steric effect between the rotor phenyl ring and N-proton in the 
trans- configuration, the cis- $\mathbf{3 4}$ is $2.74 \mathrm{kcal} \mathrm{mol}^{-1}$ more stable than its trans-form. Under irradiation with blue light $(\lambda=442 \mathrm{~nm})$, the cis- $\mathbf{3 4}$ can be switched to trans-form, the reverse conversion was triggered either by $\lambda=340 \mathrm{~nm}$ irradiation, or through an extreme slow process. The energy barrier of the trans-to-cis isomerization was determined to be $32.8 \pm 0.6$ kcal mol ${ }^{-1}$ by temperature dependency experiments over $90{ }^{\circ} \mathrm{C}$ in toluene solution, which indicated to an extraordinary long half life time ( 2700 years $)$ at $25^{\circ} \mathrm{C}$ according to Arrhenius' equation. Even in a DMSO solution, with lower $\Delta \mathrm{H}$ and larger negative $\Delta \mathrm{S}$, which results in disruption of intramolecular H-bond and more solvent reorganization, it still shows a halflife time of $\sim 265$ years.

\subsection{Cyclic azobenzenes}

In cyclic molecules, the motion of functional groups is limited by the intramolecular tension from rigid structure. In the cyclic azobenzene dimers, the isomerization undergoes through three states: (trans-, trans-), (trans-, cis-) and (cis-, cis-). At its (cis-, cis-) state, the thermal relaxation is hindered by the rigid cyclic structure, whereas the (cis-, cis-) to (trans-, cis-) step is normally much slower than the (trans-, cis-) to (trans-, trans-) step. Rau and coworkers developed azophane molecules, where two-azobenzene moieties were connected by $-\mathrm{CH}_{2}-\mathrm{S}-\mathrm{CH}_{2}$ - or $-\mathrm{CH}_{2}-\mathrm{CR}_{2}-\mathrm{CH}_{2}$ - bridges on the para- position to the $\mathrm{N}=\mathrm{N}$ bond to form cyclic dimers 35a and 35 $\mathbf{b}^{[123]}$. Both 35a and 35b showed a half-life time about 400 days in the (cis-, cis-) form. The kinetic of (cis-, cis-) to (trans-, cis-) relaxation was about 200 times slower than the (trans-, cis-) to (trans-, trans-) conversion. Yamaoki and co-workers reported xanthene-based cyclic azobenzene $\mathbf{3 6}^{[124]}$, which could form Hinge-like motion on molecular 
level during the switching process between the (trans-, trans-) state and (cis-, cis-) state. With a very rigid structure, the (cis-, $\left.c i s^{-}\right)$to (trans-, $\left.c i s^{-}\right)$step was very slow with a half-life time about 4.46 years, which was around 1000000 times slower than the (trans-, cis-) to (trans-, trans-) step with a half-life time for $1.89 \min [$ Figure 2.21].

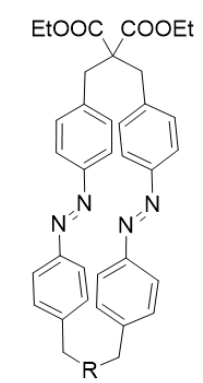

35a: $R=S$

35b: $\mathrm{R}=\mathrm{C}(\mathrm{COOEt})_{2}$

for both molecules

$\mathrm{t}_{1 / 2}=400$ days

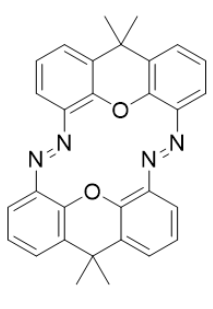

$36 t_{1 / 2}=4.46$ years

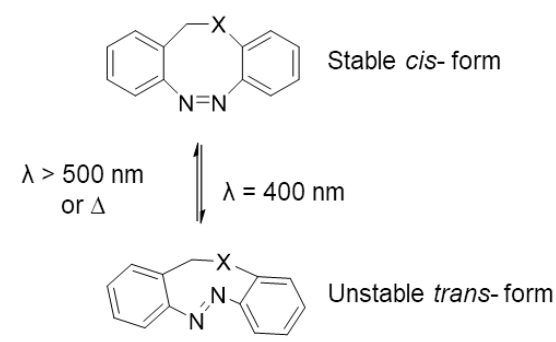

37a $\mathrm{X}=\mathrm{CH}_{2} \quad \mathrm{t}_{1 / 2}=4.5$ hours

$37 \mathrm{~b} \quad \mathrm{X}=0 \quad \mathrm{t}_{1 / 2}=89 \mathrm{sec}$

37c $X=S \quad t_{1 / 2}=3.5$ days

Figure 2.19. Cyclic azobenzenes $35 \mathbf{a}-35 \mathbf{b}, 36$ and $37 \mathbf{a}-37 \mathbf{c}$.

Another type of molecules that applied the intramolecular rigidity is diazocine 37, which was reported by Herges and co-workers ${ }^{[125,126]}$. The carbon atoms on 2- and 2'- position to the $\mathrm{N}=\mathrm{N}$ bond were connected covalently by a $-\mathrm{X}-\mathrm{CH}_{2}$ - bridge $\left(\mathrm{X}=\mathrm{CH}_{2}, \mathrm{O}\right.$ or $\left.\mathrm{S}\right)$. Due to the intramolecular tension, the $\mathbf{3 7}$ performed $c i s$-form as stable conformation, which is about 6 $\mathrm{kJ} \mathrm{mol}^{-1}$ more stable than the trans- form. The cis-to-trans conversion can be triggered by violet light at $400 \mathrm{~nm}$, and the reverse conversion undergoes with an input light wavelength $\lambda>500 \mathrm{~nm}$ or under thermal condition. The half-life of trans-to-cis isomerization was 4.5 hours for $\mathbf{3 7 a}, 89 \mathrm{sec}$ for $\mathbf{3 7 b}$ and 3.5 days for $\mathbf{3 7 c}$. Considering the size of atoms, $\mathrm{O}<\mathrm{C}<$ $\mathrm{S}$, the 37b may contain highest intramolecular tension with highest thermal relaxation rate, while the 37c had the lowest tension and showed the slowest thermal relaxation[Figure 2.21]. 
a)<smiles>O=C(CCl)Nc1ccc2c(c1)CCc1cc(NC(=O)CCl)ccc1N=N2</smiles>

$38 \mathrm{a}$<smiles>CC(=O)Nc1ccc2c(c1)CCc1cc(NC(C)=O)ccc1N=N2</smiles>

$38 \mathrm{~b}$ b)

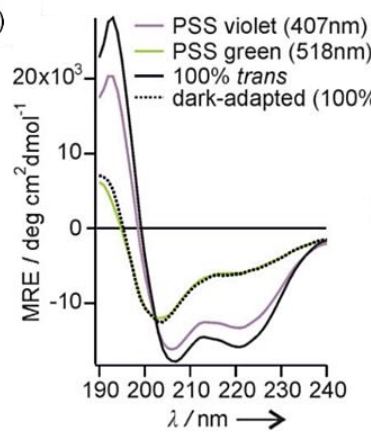

c)

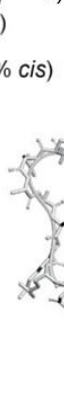

)

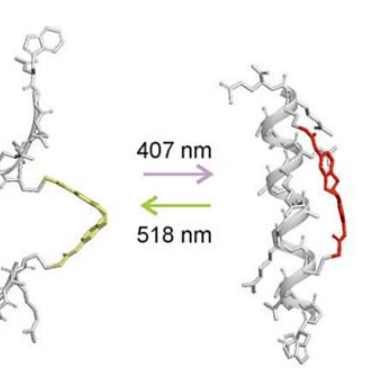

Figure 2.22. a) Chemical structure of bridged diazocine 38a and $\mathbf{3 8 b}$. b) CD spectra of $\mathbf{3 8 a}$ cross-linked FK-11. c) Models showing 38a cross-linked FK-11 in cis (left) and trans (right) conformations. Adapted with permission from Ref [127]. Copyright 2012 Wiley-VCH.

The bridged diazocine was also applied to control the peptide helical folding. Samanta et al. reported diazocine compound $\mathbf{3 8 \mathbf { a }}$ and reference molecule $\mathbf{3 8 b}, \mathbf{3 8 a}$ was applied to peptide FK11 ${ }^{[95,127]}$ [Figure 2.22a]. Both 38a and 38b performed cis- isomer as the stable form, cisto-trans isomerization occurred upon violet light irradiation $(\lambda=407 \mathrm{~nm})$, and reversely upon green light irradiation $(\lambda=518 \mathrm{~nm})$. In CD spectra of the 38a cross-linked FK11, an increased helicity after cis-to-trans isomerization was observed. Which suggested a coil like conformation of FK-11 with cis- 38a and helical conformation with trans- 38a [Figure 2.22b, 2.22c]. Noteworthy, the $\mathbf{3 8 b}$ in solution showed faster trans-to-cis thermal relaxation than 38a in conjugation with peptide ( 4.8 hours vs 8.3 hours at $\left.20^{\circ} \mathrm{C}\right)$, which suggested that helical conformation of the peptide was perhaps thermally more stable than its coil like conformation, and the trans- 38a was stabilized by the peptide helicity. This result is also in agreement with above mentioned FK11 folding experiments with compound 19 and $\mathbf{2 0}^{[127]}$. 


\subsection{Perspectives}

We have reviewed several strategies that allow designing azobenzene switches with a stable cis-isomer. Studying bi-stable azobenzenes will allow gaining insights into the switching mechanism i.e. the interplay between kinetic and thermal dynamic parameters during the photo-isomerization process. Practically, they will contribute to addressing challenges e.g. compatibility with biological condition, liquid crystal phase or solid/liquid/gas/colloidal interfaces. Overcoming these challenges is expected to contribute to the development of versatile photo-responsive materials and devices.

\subsection{References}

[1] H. Zhao, S. Sen, T. Udayabhaskararao, M. Sawczyk, K. Kučanda, D. Manna, P. K. Kundu, J-W. Lee, P. Král, R. Klajn, Nature Nanotech., 2016, 11, 82.

[2] T. H. Ware, M. E. McConney, J. J. Wie, V. P. Tondiglia, T. J. White, Science, 2015, 347, 982.

[3] T. Kudernac, N. Ruangsupapichat, M. Parschau, B.Maciá, N. Katsonis, S. R. Harutyunyan, K-H. Ernst, B. L. Feringa, Nature, 2011, 479, 208.

[4] N. Katsonis, T. Kudernac, M. Walko, S. J. van den Molen, B. J. van Wees, B. L. Feringa, Adv. Mater. 2006, 18, 1397.

[5] C. Jia, A. Migliore, N. Xin, S. Huang, J. Wang, Q. Yang, S. Wang, H. Chen, D. Wang, B. Feng, Z. Liu, G. Zhang, D-H. Qu, H. Tian, M. A. Ratner, H. Q. Xu, A. Nitzan, X. Guo, Science, 2016, 352, 1443.

[6] S. J. Asshoff, S. Iamsaard, A. Bosco, J. J. L. M. Cornelissen, B. L. Feringa, N. Katsonis, Chem. Commun., 2013, 49, 4256.

[7] L. Wang, Q. Li, Adv. Funct. Mater, 2016, 26, 10.

[8] J. W. Steed, D. R. Turner, K Wallace, Core Concepts in Supramolecular Chemistry and Nanochemistry, John Wiley \& Sons, Ltd, 2007. 
[9] M-M. Russew, S. Hecht, Adv. Mater, 2010, 22, 3348.

[10] H. Bouas-Laurent, H. Dürr, Pure Appl. Chem. 2001, 73, 639.

[11] B. L. Feringa, W. R. Browne, Molecular Switches, Second Edition, Wiley-VCH: Weinheim, 2011.

[12] S. Yagai, A. Kitamura, Chem. Soc. Rev, 2008, 37, 1520.

[13] J. M. Abendroth, O. S. Bushuyev, P. S. Weiss, C. J. Barrett, ACS Nano, 2015, 9, 7746.

[14] M. Baroncini, G. Bergamini, Chem. Rec., 2017, 17, 700.

[15] E. Merino, Chem. Soc. Rev., 2011, 40, 3835.

[16] W. Szymanski, J. M. Beierle, H. A. V. Kistemaker, W. A. Velema, B. L. Feringa, Chem. Rev., 2013, 113, 6114.

[17] A. A. Beharry, G. A. Woolley, Chem. Soc. Rev, 2011, 40, 4422.

[18] J. Mamiya, K. Kanie, T. Hiyama, T. Ikeda, T. Kato, Chem. Commun., 2002, 38, 1870.

[19] W. A. Velema, M. C. A. Stuart, W. Szymanski, B. L. Feringa, Chem. Commun., 2013, 49, 5001.

[20] D. H. Qu, Q. C. Wang, Q. W. Zhang, X. Ma, H. Tian, Chem. Rev., 2015, 115, 7543.

[21] R. Eelkema, Liquid Crystal, 2011, 38, 1641.

[22] Y. Wang, Q. Li, Adv. Mater., 2012, 24, 1926.

[23] N. Katsonis, E. Lacaze, A. Ferrarini, J. Mater. Chem., 2012, 22, 7088.

[24] R. Klajn, J. F. Stoddart, B. A. Gryzbowski, Chem. Soc. Rev., 2010, 39, 2003.

[25] X. Yao, T. Li, J. Wang, X. Ma, H. Tian, Adv. Optical Mater, 2016, 4, 1322.

[26] M. Gerth, I. K. Voets, Chem. Commun., 2017, 53, 4414.

[27] J. Dokić, M. Gothe, J. Wirth, M. V. Peters, J. Schwarz, S. Hecht, P. Saalfrank. J. Phys. Chem. A, 2009, 113, 6763.

[28] E. Mattia, S. Otto, Nat. Nanotech., 2015, 10, 111.

[29]S. A. P. van Rossum, M. Tena-Solsona, J. H. van Esch, R. Eelkema, J. Boekhoven, Chem. Soc. Rev., 2017, 46, 5519.

[30] D. Bléger, J. Schwarz, A. M. Brouwer, S. Hecht, J. Am. Chem. Soc., 2012, 134, 20597.

[31] C. Knie, M. Utecht, F. Zhao, H. Kulla, S. Kovalenko, A. M. Brouwer, P. Saalfrank, S. Hecht, D. Bléger, Chem. Eur. J., 2014, 20, 16492.

[32] J. Garcia-Amoros, D. Velasco, Beistein J. Org. Chem., 2012, 8, 1003.

[33] M. Dong, A. Babalhavaeji, S. Samanta, A. A. Beharry, G. A. Wooley, Acc. Chem. Res., 2015, 48, 2662.

[34] M. Cigl, A. Bubnov, M. Kašpar, F. Hampl, V. Hamplová, O. Pacherová, J. Svoboda, J. Mater. Chem., C, 2016, 4, 5326. 
[35] C. Hansch, A. Leo, R. W. Taft, Chem. Rev., 1991, 91, 165.

[36] J. Morene, M. Gerecke, L. Grubert, S. A. Kovalenko, S. Hecht. Angew. Chem. Int. Ed., 2016, 55, 1544 .

[37] F. Zhao, L. Grubert, S. Hecht, D. Bléger, Chem. Commun., 2017, 53, 3323.

[38] Z. Mahimwalla, K. G. Yager, J. Mamiya, A. Shishido, A. Priimagi, C. J. Barret, Poluym. Bull., 2012, 69, 967.

[39] L. Yu, M. Nakano, T. Ikeda, Nature, 2003, 425, 145.

[40] Y T. Ikeda, M. Nakano, Y. L. Yu, O. Tsutsumi, A. Kanazawa, Adv. Mater., 2003, 15, 201.

[41] O. M. Tanchak, C. J. Barrett, Macromolecules, 2005, 38, 10566.

[42] O. S. Bushuyev, A. Tomberg, T. Friščić, C. J. Barrett, J. Am. Chem. Soc., 2013, 135, 12556.

[43] M. Tsuda, K. Kuratani, Bull. Chem. Chem. Soc. Jpn., 1964, 37, 1284.

[44] P. G. de Gennes, Phys. Lett., 1969, A28, 725.

[45] P. G. de Gennes, C. R. Acad. Sci. B, 1975, 281, 101.

[46] P. G. de Gennes, Angew. Chem. Int. Ed. Engl., 1992, 31, 842.

[47] H. Finkelmann, J. H. Koch, G. Rehage, Macromol. Rapid Commun., 1981, 2, 317.

[48] J. Küpfer, H. Finkelmann, Macromol. Rapid Commun., 1991, 12, 717.

[49] D. Corbett, M. Warner, Liquid Crystals, 2009, 36, 1263.

[50] C. L. van Oosten, K. D. Harris, C. W. M. Bastiaansen, D. J. Broer, Eur. Phys. J. E, 2007, $23,329$.

[51] C. L. van Oosten, C. W. M. Bastiaansen, D. J. Broer, Nature Mater., 2009, 8, 677.

[52] K. M. Lee, M. L. Smith, H. Koerner, N. Tabiryan, R. A. Vaia, T. J. Bunning, T. J. White, Adv. Funct. Mater., 2011, 21, 2913.

[53] H. Koerner, T. J. White, N. V. Tabiryan, T. J. Bunning, R. A. Vaia, Mater. Today, 2008, 11,34 .

[54] S. Iamsaard, S. J. Aßhoff, B Matt, T. Kudernac, J. J. L. M. Cornelissen, S. P. Fletcher, N. Katsonis, Nature Chem., 2014, 6, 229.

[55] S. Iamsaard, E. Villemin, F. Lancia, S. J. Aßhoff, S. P. Fletcher, N. Katsonis, Nat. Protoc., 2016, 11,1788 .

[56] S. Iamsaard, E. Anger, S. J. Asshoff, A. Depauw, S. Fletcher, N. Katsonis, Angew. Chem. Int. Ed., 2016, 55, 9908 .

[57] K. Kumar, C. Knie, D. Bléger, M. A. Peletier, H. Friedrich, S. Hecht, D. J. Broer, M. G. Debije, A. P. H. J. Schenning, Nat. Commun., 2016, 7, 11975.

[58] R. Eelkema, B. L. Feringa, Org. Biomol. Chem., 2006, 4, 3729. 
[59] H. K. Bisoyi, Q. Li, Acc. Chem. Res., 2014, 47, 3184.

[60] Q. Li, L. Green, N. Venkataraman, I. Shiyanovskaya, A. Khan, A. Urbas, J. W. Doane, J. Am. Chem. Soc., 2007, 129, 12908.

[61] J. Ma, Y. Li, T. White, A. Urbas, Q. Li, Chem. Commun., 2010, 46, 3463.

[62] L. Green, Y. Li, T. White, A. Urbas, T. Bunning, Q. Li. Org. Biomol. Chem., 2009, 7 , 3930.

[63] H. Huang, T. Orlova, B. Matt, N. Katsonis, Macromol. Rapid Commun., 2017, 1700387.

[64] M. Brehmer, J. Lub, P. van de Witte, Adv. Mater., 1998, 10, 1438.

[65] Q. Li, Y. Li, J. Ma, D-K. Yang, T. J. White, T. J. Bunning, Adv. Mater., 2011, 23, 5069.

[66] D. J. Broer, J. Lub, G. N. Mol, Nature, 1995, 378, 467.

[67] L. Qin, W. Gu, J. Wei, Y. Yu, Adv. Mater., 2017, 1704941.

[68] A. Harada, Y. Takashima, M. Nakahata, Acc. Chem. Res., 2014, 47, 2128.

[69] H. M. D. Bandara, S. C. Burdette, Chem. Soc. Rev., 2012, 41, 1809.

[70] A. Ueno, K. Takahashi, T. Osa, J. Chem. Soc. Chem. Comm., 1980, 17, 837.

[71] Y. Liu, C. Yu, H. Jin, B. Jiang, X. Zhu, Y. Zhou, Z. Lu, D. Yan, J. Am. Chem. Soc., 2013, $135,4765$.

[72] P. Wan, Y. Wang, Y. Jiang, H. Xu, X. Zhang, Adv. Mater., 2009, 21, 4362.

[73] O. Roling, L. Stricker, J. Voskuhl, S. Lamping, B. J. Ravoo, Chem. Commun., 2016, 52, 1964.

[74] G. Yu, K. Jie, F. Huang, Chem. Rev., 2015, 115, 7240.

[75] A. Samanta, M. C. A. Stuart, B. J. Ravoo, J. Am. Chem. Soc., 2012, 134, 19909.

[76] H. Yamaguchi, Y. Kobayashi, R. Kobayashi, Y. Takashima, A. Hashidzume, A. Harada, Nat. Commun., 2012, 3, 603.

[77] J. Lagona, P. Mukhopadhayay, S. Chakrabarti, L. Isaacs, Angew. Chem. Int. Ed., 2005, $44,4844$.

[78] F. Tian, D. Jiao, F. Biedermann, O. A. Scherman, Nat. Commun., 2012, 3, 1207.

[79] C.Stoffelen, J. Voskuhl, P. Jonkheijm, J. Huskens, Angew. Chem. Int. Ed., 2014, 53, 3400. [80] J. del Barrio, P. N. Horton, D. Lairez, G. O. Lloyd, C. Toprakcioglu, O. A. Scherman, J. Am. Chem. Soc., 2013, 135, 11760.

[81] H. B. Cheng, Y. M. Zhang, C. Xu, Y. Liu, Sci, Rep., 2014, 4, 4210.

[82] L. Zhang, H. Zhang, F. Gao, H. Peng, Y. Ruan, Y. Xu, W. Weng. RSC Adv, 2015, 5, 12007.

[83] H. Huang, A. Juan, N. Katsonis, J. Huskens, Tetrahedron, 2017, 73, 4913.

[84] W. A. Velema, J. P. van der Berg, M. J. Hansen, W, Szymanski, A. J. M. Driessen, B. L. 
Feringa, Nature Chem., 2013, 5, 924.

[85] M. M. Lerch, M. J. Hansen, G. M. van Dam, W. Szymanski, B. L. Feringa, Angew. Chem. Int. Ed., 2016, 55, 10978.

[86] Y. Matsumura, H. N. Ananthaswamy, Toxicol. Appl. Pharmacol., 2004, 195, 298.

[87] K. Kalka, H. Merk, H. Mukhtar, J. Am. Acad. Dermatol., 2000, 42, 389.

[88] R. Weissleder, Nat. Biotechnol., 2001, 19, 316.

[89] M. Wegener, M. J. Hansen, A. J. M. Driessen, W. Szymanski, B. L. Feringa, J. Am. Chem. Soc., 2017, 139, 17979.

[90] G. H. Hitchings, G. B. Elion, H. van der Werff, E. A. Falco, J. Biol. Chem., 1948, 174, 765 .

[91] B. Roth, E. A. Falco, G. H. Hitchings, S. R. M. Bushby, J. Med. Pharm. Chem., 1962, $5,1103$.

[92] N. Nishimura, T. Sueyoshi, H. Yamanaka, E. Imai, S. Yamamoto, S. Hasegawa, Bull. Chem. Soc. Jpn., 1976, 49, 1381.

[93] N. Nishimura, T. Tanaka, M. Asano, Y. Sueishi, J. Chem. Soc. Perkin Trans. 2, 1986, 1839-1845.

[94] A. A. Beharry, O. Sadovski, G. A. Woolley, J. Am. Chem. Soc., 2011, 133, 19684.

[95] S. Samanta, A. A. Beharry, O. Sadovski, T. M. McCormick, A. Babalhavaeji, V. Tropepe, G. A. Woolley, J. Am. Chem. Soc., 2013, 135, 9777.

[96] D. Wang, M. Wagner, H-J. Butt, S. Wu, Soft Matter, 2015, 11, 7656.

[97] D. Wang, S. Wu, Langmuir, 2016, 32, 632.

[98] P. Weis, D. Wang, S. Wu, Macromol., 2016, 49, 6368.

[99] P. J. Coelho, L. M. Carvalho, A. M. C. Fonseca, M. M. M. Raposo, Tetrahedron Lett., 2006, 47, 3711 .

[100] P. J. Coelho, L. M. Cavalho, J. C. V. P. Moura, M. M. M. Raposo, Dyes Pigm., 2009, $82,130$.

[101] J. Garcia-Amorós, M. C. R. Castro, P. Coelho, M. M. M. Raposo, D. Velasco, Chem. Commun., 2013, 49, 11427.

[102] J. Garcia-Amorós, A. Bučinskas, M. Reig, S. Nonell, D. Velasco, J. Mater. Chem. C, 2014, $2,474$.

[103] T. Wendler, C. Schütt, C. Näther, R. Herges, J. Org. Chem., 2012, 77, 3284.

[104] C. E. Weston, R. D. Richardson, P. R. Haycock, A. J. P. White, M. J. Fuchter, J. Am. Chem. Soc., 2014, 136, 11878.

[105] J. Calbo, C. E. Weston, A. J. P. White, H. S. Rzepa, J. Contreras-García, M. J. Fuchter, 
J. Am. Chem. Soc., 2017, 139, 1261.

[106] L. Stricker, E. Fritz, M. Peterlechner, N. L. Doltsinis, B. J. Ravoo, J. Am. Chem. Soc., 2016, 138, 4547.

[107] S. Sagebiel, L. Stricker, S. Engel, B. J. Ravoo, Chem. Commun., 2017, 53, 9296.

[108] N. Möller, T. Hellwig, L. Stricker, S. Engel, C. Fallnich, B. J. Ravoo, Chem. Commun., 2017, 53, 240.

[109] F. Azuel, Chem. Rev., 2004, 104, 139; J. A. Capobianco, F. Vetrone, T. D’Alesio, G.

Tessari, A. Speghini, M. Bettinelli, Phys. Chem. Chem. Phys., 2000, 2, 3203.

[110] P. Courtot, J. Le Saint, R. Pichon, Bull. Soc. Chim. Fr. 1975, 2538, 2542.

[111] P. Courtot, R. Pichon, J. Lesaint, Tetrahedron Lett., 1976, 17, 1181.

[112] R. Pchon, J. Le Saint, P. Courtot, Tetrahedron, 1981, 37, 1517.

[113] S. M. Landge, I. Aprahamian, J. Am. Chem. Soc., 2009, 131, 18269.

[114] S. M. Landge, E. Tkatchouk, D. Benítez, D. A. Lanfranchi, M. Elhabiri, W. A. Goddard, III, I. Aprahamian, J. Am. Chem. Soc., 2011, 133, 9812.

[115] I. Aprahamian, Chem. Commun., 2017, 53, 6674.

[116] Y. Yang, X. Su, C. N. Carroll, I. Aprahamian, Chem. Sci., 2012, 3, 610.

[117] Y. Yang, R. P. Hughes, I. Aprahamian, J. Am. Chem. Soc., 2012, 134, 15221.

[118] Y. Yang, R. P. Hughes, I. Aprahamian, J. Am. Chem. Soc., 2014, 136, 13190.

[119] H. M. D. Bandara, S. C. Burdette, Chem. Soc. Rev., 2012, 41, 1809.

[120] A. A. Blevins, G. J. Blanchard, J. Phys. Chem. B, 2004, 108, 4962.

[121] H. Wisn, Y. Y. Wang, D. S. Guo, I. Aprahamian, J. Am. Chem. Soc., 2017, 139, 1037.

[122] H. Qian, S. Pramanik, I. Aprahamian, J. Am. Soc. Chem., 2017, 139, 9140.

[123] D. Röttger, H. Rau, J. Photochem. Photobil., A, 1996, 101, 205.

[124] S. A. Nagamani, Y. Norikane, N. Yamaoki, J. Org. Chem., 2005, 70, 9304.

[125] R. Siewertsen, H. Neumann, B. Buchheim-Stehn, R. Herges, C. Näther, F. Renth, F. Temps, J. Am. Chem. Soc., 2009, 131, 15594.

[126] M. Hammerich, C. Schütt, C. Stähler, P. Lentes, F. Röhricht, R. Höppner. R. Herges, J. Am. Chem. Soc., 2016, 138, 13111.

[127] S. Samanta, C. Qin, A. J. Lough, G. A. Woolley, Angew. Chem. Int. Ed., 2012, 51, 6452. 


\section{Chapter 3}

\section{Long-lived supramolecular helices promoted by fluorinated photo-switches}

Chiral azobenzenes can be used as photo-switchable dopants to control supramolecular helices in liquid crystals. However, the lack of thermal stability of the cis-isomer precludes envisioning the generation of long-lived supramolecular helices with light. Here we demonstrate thermally stable and axially chiral azobenzene switches that can be used as chiral dopants to create supramolecular helices from (achiral) nematic liquid crystals. Their trans-to-cis photo-isomerization leads to a variation of helical twisting power that reaches up to $60 \%$, and the helical superstructure that is engineered with light displays a relaxation time that reaches tens of hours. These results demonstrate that combining ortho-fluorination with axial chirality in molecular photo-switches constitutes an efficient strategy to promote long-lived helical states and further, this approach shows potential to design supramolecular machines that are controlled by light entirely.

This chapter is published in H. Huang, T. Orlova, B. Matt, N. Katsonis, Macromol. Rapid Commun., 2017, 1700387 


\subsection{Introduction}

Molecular photos-switches are dynamic and versatile building blocks for the design of wholly synthetic supramolecular ${ }^{[1]}$ and macromolecular machines ${ }^{[2-3]}$. However, making full use of their potential for the design and synthesis of wholly synthetic soft machines will require the development of strategies by which their photo-isomerization is amplified from the molecular level across length scales, and ultimately up to the macroscopic level, by taking advantage of cooperative effects ${ }^{[4-5]}$. Liquid crystals provide versatile, sensitive and anisotropic supramolecular matrices that respond with collective effects to small changes in their composition and thus they can amplify molecular chirality and motion efficiently into optical or mechanical responses $^{[6]}$. Coupling molecular photo-switches with liquid crystals has allowed developing light-responsive molecular systems with applications ranging from optical memory writing ${ }^{[7]}$ to colourful displays ${ }^{[8]}$. Combined with (helix-based) cholesteric liquid crystals, molecular photo-switches have allowed creating and manipulating chiral topological structures ${ }^{[9-10]}$, changing reflexion colours and polarization ${ }^{[11-13]}$, detecting catalytic reactions ${ }^{[14]}$ and developing soft rotating devices ${ }^{[5]}$. When embedded in liquid crystal networks covalently, molecular photo-switches have mediated complex shape transformations ${ }^{[15-16]}$, and the conversion of light into of work and power by soft polymer machines has been demonstrated also. ${ }^{[17]}$

Light-responsive cholesteric liquid crystals can be prepared by dissolving a small percentage of chiral photo-switches in a nematic (achiral) liquid crystal host ${ }^{[4,18]}$ [Figure 3.1]. The propensity of these photo-switches, used as dopants, to induce a 
twist in a given host is characterized by their helical twisting power (HTP), defined as HTP $=(p \times c \times e e)^{-1}$, where $p$ is the pitch and corresponds to a full $360^{0}$ rotation of the molecules along the helical axis, $c$ is the concentration of the dopant in wt $\%$ and $e e$ is the enantiomeric excess of the dopant. Chiral switches are typically characterised by large twisting powers, for their chirality can be amplified across length scales effectively ${ }^{[6]}$.
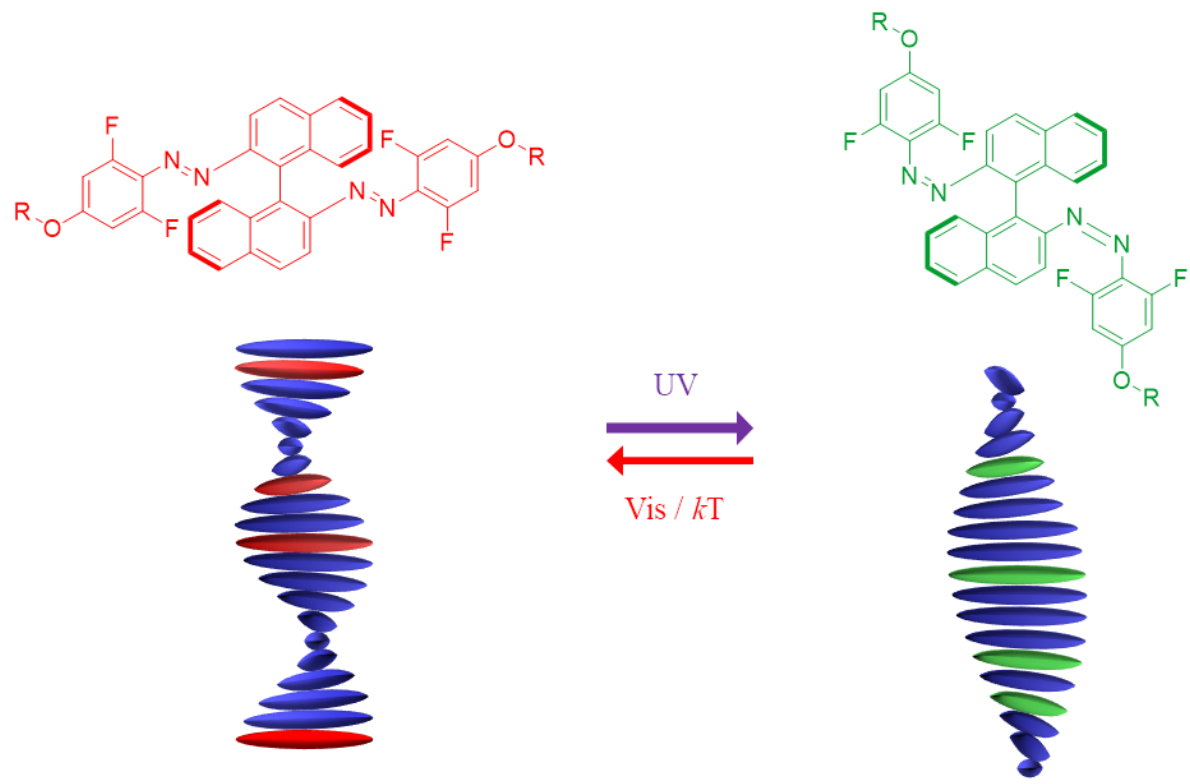

Figure 3.1. A photo-switch with axial chirality allows forming a cholesteric liquid crystal, and controlling its helix-based structure with light.

Azobenzenes are typically used as switchable dopants because their trans/cis photo-isomerization is associated with large changes in both the geometry and the dipole moment of the molecule. While rod-like trans-azobenzenes are compatible with the nematic order, the bent-like cis-molecules disturb this molecular order significantly. Consequently, the helical twisting power of the trans and the cis isomers 
are remarkably different. Moreover, coupling azobenzenes to elements of axial chirality yields both a large helical twisting power in the ground state and a large variation of helical twisting power under irradiation with light, compared to chiral azobenzenes that display point chirality only ${ }^{[11,19-22]}$. Light-driven helix inversion has also been reported, when chiral azobenzene dopants with axial chirality were used as

dopants $^{[23-25]}$. However, the lack of thermal stability of the cis-isomer remains a limitation to reaching the full potential of complex dynamic behaviour in these supramolecular systems.

Fluorinated azobenzenes display larger thermal stability than their classical counterparts, primarily because the repulsion between the nitrogen lone pairs that destabilises the cis-isomer is lifted by electron-withdrawing effect of the fluorine atoms in ortho-position ${ }^{[26-29]}$. Here, we combine the axial chirality of a binaphthyl moiety with ortho-fluorination of an azobenzene moiety, in molecular photo-switches that induce supramolecular helices in liquid crystals in both forms, and thus we demonstrate the photo-engineering of long-lived supramolecular helices in soft matter.

\subsection{Results and discussion}

Molecules 1a and 1b were synthesized from commercially available starting materials. Their photochemistry and performance as dopants were investigated by comparing them to the reference azobenzenes $\mathbf{2} \mathbf{a}^{[19]}$ and $\mathbf{2} \mathbf{b}^{[20,30]}$ in the same conditions [Figure 3.2, Figure S3.1]. The chemical structures of the intermediate and final 
compounds were identified by ${ }^{1} \mathrm{H}$ NMR, ${ }^{13} \mathrm{C}$ NMR and high-resolution mass spectrometry.

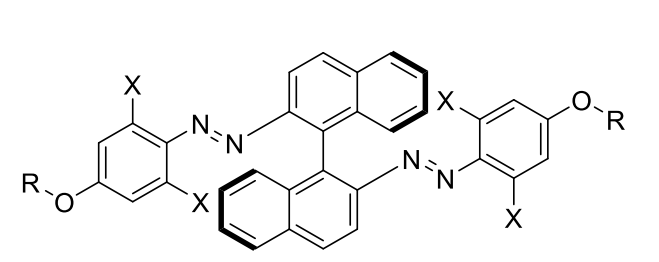

1a: $X=F, R=$<smiles>CCCCCCCCCCCCC(C)C</smiles>

2a: $X=H, R=$<smiles>CCCCCCCCCCCCCCCC(C)C</smiles>

Figure 3.2. Molecular structure of the fluorinated photo-switches used as dopants, and their classical counterpart.

Solutions of 1 and 2 in chloroform were irradiated with UV light $(\lambda=365 \mathrm{~nm})$, which led to trans-to-cis isomerization, as evidenced by a decrease in the $\pi \rightarrow \pi^{*}$ absorption band corresponds to the (trans, trans)-azobenzene chromophore $\left(\lambda_{\max }\right.$ a $_{\mathrm{a}}=$ $\left.348 \mathrm{~nm}, \lambda_{\max 1 \mathrm{~b}}=348 \mathrm{~nm}, \lambda_{\max 2 \mathrm{a}}=358 \mathrm{~nm}, \lambda_{\max 2 \mathrm{~b}}=356 \mathrm{~nm}\right)$ and an increase in the $\mathrm{n} \rightarrow \pi^{*}$ absorption band around $450 \mathrm{~nm}^{[26-27]}$ [Figure S3.2]. Since the dopants incorporates two azo moieties that are decoupled from each other, irradiation with light induces the formation of photo-isomers containing either one or two cis-moieties. The photo-switching sequence is thus (trans, trans $) \rightarrow($ trans, cis $) \rightarrow($ cis, cis $)$ [Figure 3.3]. Once photo-stationary state is reached, $27 \%$ of the azobenzene moieties have switched into the cis-form for $\mathbf{1 a}$, and $40 \%$ for $\mathbf{1 b}$. These photo-stationary ratios 
compare well with the ratios we measured for $\mathbf{2 a}$ and $\mathbf{2 b}$ [Figure S3.3], while remaining moderate. The UV-vis spectra show that the $\mathrm{n} \rightarrow \pi^{*}$ absorption band of $\mathbf{1 a}$ and $\mathbf{1 b}$ do not shift during the photo-isomerization [Figure S3.2], which means the $\mathrm{n} \rightarrow \pi^{*}$ transition bands of the trans- and cis- isomers are not separated, likely due to the combined contribution from ortho-fluorine atoms and the naphthalene group. Due to this similarity in excitation energies, it is not possible to switch dopants $\mathbf{1 a}$ and $\mathbf{1 b}$ in both directions with visible light ${ }^{[27]}$. However, while fluorination does not lift the similarity between the $\mathrm{n} \rightarrow \pi^{*}$ transition of the trans-form and the cis-form, its effect is visible in the blue-shift of the $\pi \rightarrow \pi^{*}$ absorption bands of fluorinated $\mathbf{1 a}$ and $\mathbf{1 b}$, compared to their classical counterparts $\mathbf{2 a}$ and $\mathbf{2 b}(\sim 10 \mathrm{~nm})$. We conclude that an electron-withdrawing effect does take place by partial ortho-fluorination, and this effect is likely to stabilize the cis-form by minimizing the repulsion between lone pairs. We have investigated the cis-to-trans relaxation kinetics to confirm the thermal stabilisation. 


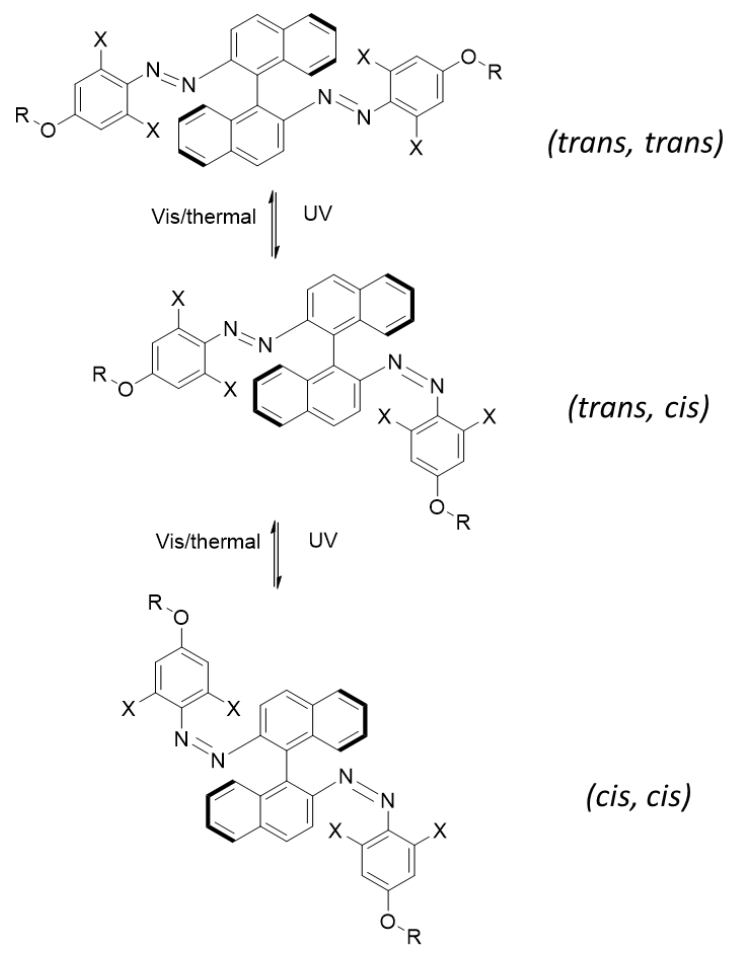

Figure 3.3. Photo-switching sequence for the chiral molecules used as dopants.

Monitoring of the increasing in $\pi \rightarrow \pi^{*}$ absorption band under thermal relaxation shows that, in both molecular switches, the cis-to-trans relaxation follows first order kinetics. As expected from the presence of fluorine atoms, the thermal relaxation of cis-1 is slower than that of cis-2 [Table 3.1, Figure S3.4]. However, both $c i s-1 a$ and cis-1b display only a moderate thermal stability compared to the thermal stability reported for tetra-fluorinated azobenzenes ${ }^{[26-27]}$. This moderate thermal stability likely originates in the electron-donating resonance effect from the naphthyl group, as a counterpoint to the electron-withdrawing inductive effect from the fluorine atoms. 
Table 3.1. Half-life time of the thermal relaxation $t_{1 / 2}$ for the photo-switches in solution, and when used as chiral dopants in liquid crystals.

\begin{tabular}{|c|c|c|c|c|}
\hline Dopant & $\begin{array}{c}\mathrm{t}_{1 / 2}{ }^{[\mathrm{a}][\mathrm{b}]} \text { (hours) } \\
\text { in } \mathrm{CHCl}_{3}\end{array}$ & $\begin{array}{c}\text { Liquid } \\
\text { crystal }\end{array}$ & $\begin{array}{c}\mathrm{t}_{1 / 2}{ }^{[\mathrm{a}][\mathrm{b}]} \text { (hours) } \\
\text { in liquid crystal }\end{array}$ & $\begin{array}{c}\mathrm{t}_{1 / 2}{ }^{[\mathrm{a}][\mathrm{c}]} \text { (hours) } \\
\text { in liquid crystal }\end{array}$ \\
\hline \multirow{2}{*}{$\mathbf{1 a}$} & 10.1 & $\begin{array}{c}\mathrm{E} 7 \\
\text { MLC 6608 }\end{array}$ & $\begin{array}{c}35.6 \\
59.3\end{array}$ & $\begin{array}{l}28.0 \\
44.1\end{array}$ \\
\hline \multirow{2}{*}{$\mathbf{1 b}$} & 4.2 & E7 & 33.6 & 41.0 \\
& MLC 6608 & 67.9 & 93.6 \\
\hline \multirow{2}{*}{$\mathbf{2 a}$} & 1.0 & E7 & 8.5 & 3.7 \\
\hline \multirow{2}{*}{$\mathbf{2 b}$} & 0.6 & $\mathrm{E} 7$ & 5.8 & 7.9 \\
\hline
\end{tabular}

[a] all thermal relaxation half-life time were measured at $22^{\circ} \mathrm{C}$. [b] monitored by changes in UV/Vis absorption spectra. [c] monitored by changes in the cholesteric pitch, as measured from a wedge cell.

Next, the photo switches were investigated in nematic liquid crystals, starting with the commercially available mixture E7 [Figure S3.5]. In this liquid crystalline environment, UV irradiation of all four compounds results in a decrease of the $\pi \rightarrow \pi^{*}$ absorption band. The investigation of the cis-to-trans relaxation [Figure S3.6] reveals that, independently on the presence of fluorine, the half-life time of the cis-form is longer in the liquid crystalline phase than in solution. This stabilization in the liquid crystal likely originates in the combined effect of viscosity, high $\pi$-electron density and decreased molecular degree of freedom in the anisotropic environment of the liquid crystal. The suitability of $\mathbf{1 a}$ and $\mathbf{1 b}$ as chiral dopants was also investigated in MLC 6608, a liquid crystal that is nematic at room temperature with a negative dielectric anisotropy, while E7 displays a positive dielectric anisotropy. Both molecular photo-switches show longer half-life times in MLC 6608 than in E7 [Table 3.1, Figure S3.7 - S3.8]. 

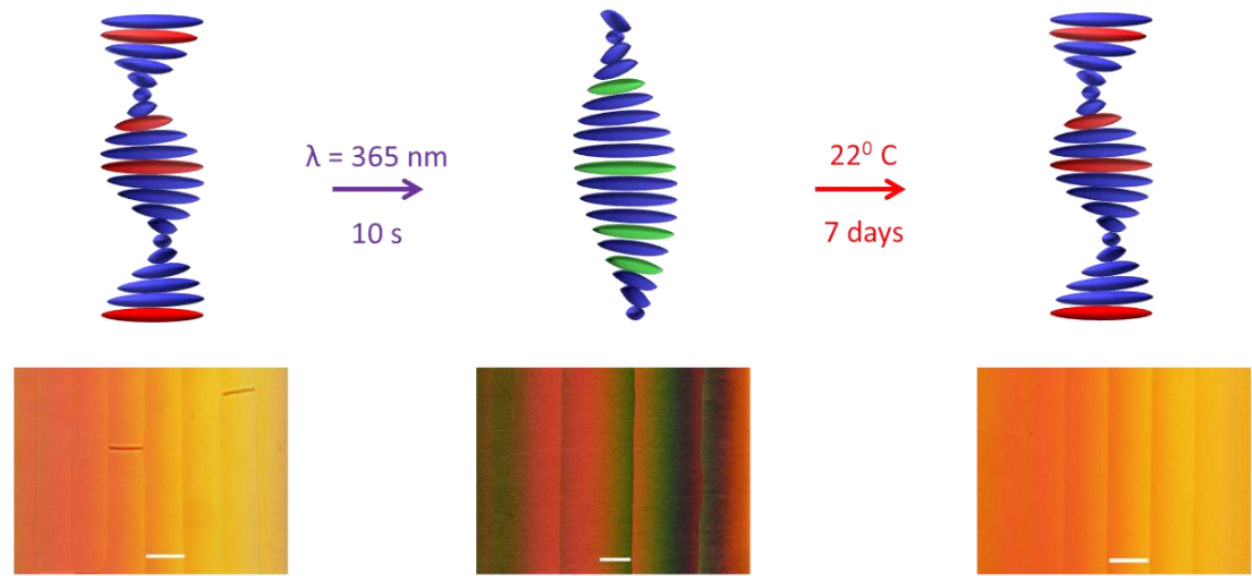

$\mathrm{HTP}=-46.3 \mu \mathrm{m}^{-1}$

$$
\mathrm{HTP}=-19.0 \mu \mathrm{m}^{-1}
$$

HTP $=-39.2 \mu \mathrm{m}^{-1}$

Figure 3.4. UV irradiation and thermal relaxation of the liquid crystal helices induced by dissolving 1 $\mathrm{wt} \%$ of photo-switch $\mathbf{1 b}$ in the nematic liquid crystal E7. (scale bar $=100 \mu \mathrm{m}$, wedge cell $\tan \theta=0.0115$ )

Table 3.2. Helical twisting power (HTP) for azobenzene dopants in the trans form and at the photo-stationary state (PSS).

\begin{tabular}{|c|c|c|c|c|}
\hline Dopant & Liquid crystal host & $\begin{array}{c}\text { HTP initial } \\
\left(\mu \mathrm{m}^{-1} \mathrm{wt} \%^{-1}\right)\end{array}$ & $\begin{array}{c}\text { HTP at PSS } \\
\left(\mu \mathrm{m}^{-1} \mathrm{wt}^{-1}\right)\end{array}$ & $\Delta$ HTP (\%) \\
\hline 1a & E7 & -20.1 & -8.1 & 60 \\
\hline 1b & MLC 6608 & -31.8 & -17.7 & 44 \\
\hline 2a & MLC 6608 & -46.3 & -19.0 & 56 \\
\hline 2b & E7 & -40.6 & -2.7 & 93 \\
\hline
\end{tabular}

The helical twisting power of the dopants was determined by using the Grandjean-Cano 
wedge cell method ${ }^{[31]}$. In all samples, the cholesteric helix was left-handed. In E7, the initial HTP values for the fluorinated dopants $\mathbf{1 a}$ and $\mathbf{1 b}$ are lower than for the corresponding reference molecules $\mathbf{2} \mathbf{a}$ and $\mathbf{2 b}$, probably because the fluorine atoms modify the dipole moment of the switches. On another hand, comparing $\mathbf{1 b}$ with $\mathbf{1 a}$, and $\mathbf{2 b}$ with $\mathbf{2 a}$, it might be that the rigid moiety enhances the transfer of chirality from the switches to the achiral nematic environment. The photo-induced variation of helical twisting power is modest in the presence of the ortho-fluorinated switches [Table 3.2]. However, the thermal relaxation of the light-induced cholesteric helix, from the photo-stationary helix back to the initial helical organization, reveals one order of magnitude slower for $\mathbf{1}$ than for $\mathbf{2}$ [Figure 3.4, Table 3.1, Figure S3.9 - S3.10]. In MLC 6608, 1a and 1b showed similar HTP values and similar amplitudes of variation after photo-isomerization, while the $\mathbf{1 b}$ showed two-times longer half-life time of pitch relaxation [Table 3.2, Figure S3.11 - S3.12].

Noticeably, for switches $\mathbf{1 a}$ and $\mathbf{2 a}$, the half-life time is smaller as measured by supramolecular pitch change, than when it is measured by molecular changes in absorbance. Alternatively, for compounds with extended rigid arms $\mathbf{1 b}$ and $\mathbf{2 b}$, the opposite was observed [Table 3.1]. In principle, the re-adjustment of the cholesteric helix, in response to molecular isomerisation, depend on the elastic properties of the liquid crystal and on the thickness of the sample ${ }^{[32]}$. Here we anticipate that the difference in response of the helices relates to different elastic properties, combined with the effects of liquid crystalline confinement. 


\subsection{Conclusions}

We have designed and synthesized ortho-fluorinated and axially chiral molecular photo-switches that can be used as dopants in liquid crystals, where they induce the formation of self-organized helical superstructures efficiently. The trans-to-cis photoisomerization of these molecules is accompanied by a variation of helical twisting power that reaches up to $60 \%$, and the supramolecular helix they generate under irradiation with light is stable thermally, with a relaxation time of tens of hours. Besides their efficiency as chiral dopants and thermal stability in both forms, we also note that in these fluorinated switches, the $\mathrm{n} \rightarrow \pi^{*}$ excitation energies are similar, therefore it is not possible to address them with visible light. The switches also show moderate photo-conversions. Overall, combining ortho-fluorination with axial chirality appears as a promising strategy to design molecular photo-switches that promote light-driven and long-lived helical states in liquid crystals. We envision that these versatile supramolecular helices can be incorporated into complex molecular systems across length scales, towards soft machines that are controlled by light entirely ${ }^{[33]}$. 


\subsection{Acknowledgements}

Dr. Tetiana Orlova is gratefully acknowledged for performing the experiments of molecule 1a and 2a in E7, as well as 1a in MLC 6608.

\subsection{Supporting information}

\subsubsection{Materials and methods}

All chemicals and solvents were purchased from commercial suppliers and used without further purification.

${ }^{1} \mathrm{H}$ NMR and ${ }^{13} \mathrm{C}$ NMR spectra were recorded in $\mathrm{CDCl}_{3}$ in a Bruker $400 \mathrm{MHz}$ Avance III NMR spectrometer. Chemical shifts are in $\delta$ units (ppm) with the residual solvent peak as the internal standard. The coupling constant $(J)$ is reported in hertz $(\mathrm{Hz})$. NMR splitting patterns are designed as follows: s, singlet; d, doublet; $\mathrm{t}$, triplet; and m, multiplet. Analytical chromatography was performed on silica gel (60-200 mesh). High resolution mass spectra were registered using Waters/Micromass LCT Mass spectrometer with Reserpine as an internal standard.

UV-visible absorption spectra were recorded using a Perkin Elmer Lambda 850 spectrophotometer. To take the spectra, $0.1 \mathrm{mM}$ solutions of $\mathbf{1 a}, \mathbf{1} \mathbf{b}$ and $\mathbf{2 a}, \mathbf{2} \mathbf{b}$ in chloroform were placed in $1 \mathrm{~cm}$ quartz cuvettes. Photo-switches 1a, 1b and 2a, 2b were incorporated into a nematic liquid crystal at $1 \mathrm{wt} . \%$ concentration, and the resulting cholesteric liquid 
crystals was introduced by capillary forces in liquid crystal cells (planar alignment, $15 \mu \mathrm{m}$ nominal gap value).

The Grandjean-Cano lines were observed in wedge cells ( $\tan \theta=0.0269$ or 0.0115$)$, by using an Olympus BX-51 polarized optical microscope. All planar and wedge LC cells are purchased from E.H.C CO., LTD (Japan).

UV irradiation ( $\lambda=365 \mathrm{~nm}$ ) was performed using a Hönle bluepoint LED lamp. The samples were placed at the distance $5 \mathrm{~cm}$ from the exit pupil of the optical fiber. The intensity of the lamp is set at $80 \%$ level from the maximum intensity level $170 \mathrm{~mW} / \mathrm{cm}^{2}$.

Irradiation by white light and quasi-monochromatic light $(\lambda=420 \mathrm{~nm}$ and $\lambda=430 \mathrm{~nm})$ was performed using an Edmund Optics MI-150 Fiber Optic Illuminator. A Meadowlark Optics IR filter was used to prevent the samples from heating. Midwest optical filter $400 \div 700$ $\mathrm{nm}$, Edmund optics filters $420 \mathrm{~nm}$ and $430 \mathrm{~nm}$ were used to obtain the desired spectral range of light. The samples were placed at the distance $5 \mathrm{~cm}$ from the exit pupil of the optical fiber. The intensity of the illuminator was set at $100 \%$ level.

Irradiation by quasi-monochromatic light $(\lambda=520 \mathrm{~nm})$ was performed using HLV2-223W Compact Spot Light controlled by PJ-1505-3CA Analog Control Unit (both are from CCS Inc.). As before, samples were placed at the distance $5 \mathrm{~cm}$ from the exit pupil while the intensity of the lamp is set at $100 \%$ level. 


\subsubsection{Synthesis and characterization}

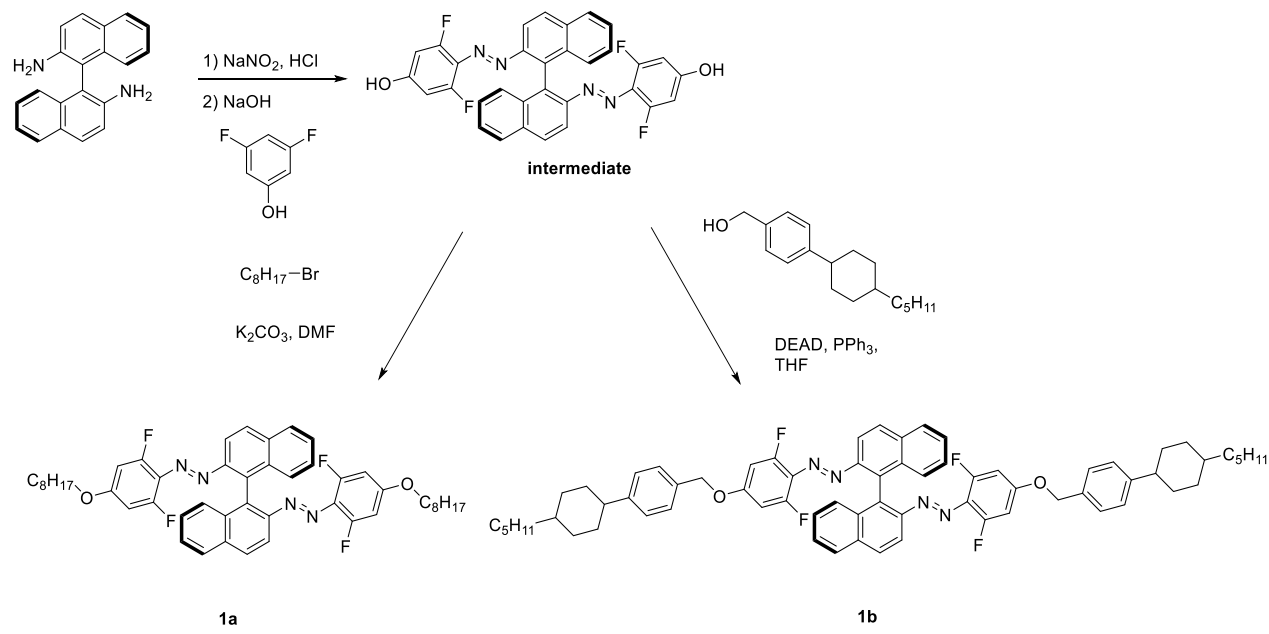

Figure S3.1. Synthesis of the fluorinated photo-switches 1a and $\mathbf{1 b .}$

(S)-(-)-4,4'-(([1,1'-binaphthalene]-2,2'-diylbis(diazene-2,1-diyl))bis(3,5-difluorophenol):

(S)-(-)-1,1'-Binaphthyl-2,2'-diamine (0.142 g, 0.5 mmol) was dissolved in a solution of $\mathrm{H}_{2} \mathrm{O}$ (2.5 mL), THF $(0.3 \mathrm{~mL})$ and concentrated $\mathrm{HCl}(0.35 \mathrm{ml})$. Next this solution is cooled down to $0^{\circ} \mathrm{C}$ in an ice water bath. A solution of sodium nitrite $(0.083 \mathrm{~g}, 0.6 \mathrm{mmol})$ in $\mathrm{H}_{2} \mathrm{O}(1.5 \mathrm{ml})$ is dropped into the solution which is being stirred. The resulting brown suspension is dropped into the second solution of 3,5-difluoro-phenol $(0.143 \mathrm{~g}, 0.55 \mathrm{mmol})$ and $\mathrm{NaOH}(0.130 \mathrm{~g}$, $3.25 \mathrm{mmol})$ in $\mathrm{H}_{2} \mathrm{O}(2 \mathrm{ml})$ at $0^{\circ} \mathrm{C}$. The final mixture is acidified with aqueous $\mathrm{HCl}$. The resulting precipitate is recovered by suction filtration to get the crude intermediate compound which is further purified by column chromatography on silica gel using the mixture of dichloromethane and methanol $(50: 1)$ as an eluent to get the red solid intermediate compound with $37 \%$ yield.

${ }^{1} \mathrm{H} \mathrm{NMR}(400 \mathrm{MHz})\left(\mathrm{CDCl}_{3}\right): \delta=6.11(\mathrm{~d}, 4 \mathrm{H}, J=12.0 \mathrm{~Hz}), 7.23(\mathrm{t}, 2 \mathrm{H}, J=8.0 \mathrm{~Hz}), 7.44(\mathrm{~m}$, 
2H), $7.48(\mathrm{~m}, 2 \mathrm{H}), 7.90(\mathrm{~d}, 2 \mathrm{H}, J=8.0 \mathrm{~Hz}), 8.01(\mathrm{~d}, 2 \mathrm{H}, J=9.0 \mathrm{~Hz}), 8.08 \mathrm{ppm}(\mathrm{d}, 2 \mathrm{H}, J=$ $9.2 \mathrm{~Hz})$.

${ }^{13} \mathrm{C}$ NMR $(100 \mathrm{MHz})\left(\mathrm{CDCl}_{3}\right): \delta=102.98,114.23,123.22,127.20,127.38,127.44,128.57$, $130.36,133.75,134.02,145.57,157.89,160.51 \mathrm{ppm}$.

TOF MS ES+ $(\mathrm{M}+\mathrm{H})$ Calcd. for $\mathrm{C}_{32} \mathrm{H}_{19} \mathrm{~F}_{4} \mathrm{~N}_{4} \mathrm{O}_{2}: 567.1439$, found 567.1423 .

(S)-(-)-2,2'-bis((E)-(2,6-difluoro-4-(octyloxy)phenyl)diazenyl)-1,1'-binaphthalene 1a: a mixture of the intermediate compound $(0.072 \mathrm{~g}, 0.126 \mathrm{mmol}), 1$-bromooctane $(0.061 \mathrm{~g}, 0.316$ mmol) and potassium carbonate $(0.044 \mathrm{~g}, 0.316 \mathrm{mmol})$ in DMF $(5 \mathrm{ml})$ is heated up to $80^{\circ} \mathrm{C}$ under $\mathrm{N}_{2}$ and stirred overnight. After cooling to room temperature, the resulting solution is diluted with ethyl acetate $(30 \mathrm{ml})$ and then washed with water 3 times. The formed organic layer is collected, dried over $\mathrm{MgSO}_{4}$ and the solvent is evaporated under reduced pressure. The residue is purified by column chromatography on silica gel using the mixture of n-hexane and ethyl acetate (30:1) as an eluent to get the red solid 1a with $44 \%$ yields.

${ }^{1} \mathrm{H} \mathrm{NMR}(400 \mathrm{MHz})\left(\mathrm{CDCl}_{3}\right): \delta=0.85(\mathrm{t}, 6 \mathrm{H}, J=7.1 \mathrm{~Hz}), 1.24(\mathrm{~b}, 24 \mathrm{H}), 1.68(\mathrm{~m}, 4 \mathrm{H}), 3.81$ (t, 4H), $6.27(\mathrm{~d}, 4 \mathrm{H}, J=12.0 \mathrm{~Hz}), 7.26(\mathrm{t}, 4 \mathrm{H}, J=8.0 \mathrm{~Hz}), 7.43(\mathrm{~m}, 2 \mathrm{H}), 7.48(\mathrm{~m}, 2 \mathrm{H}), 7.91$ $(\mathrm{d}, 2 \mathrm{H}, J=8.0 \mathrm{~Hz}), 7.99(\mathrm{~d}, 2 \mathrm{H}, J=9.0 \mathrm{~Hz}), 8.10 \mathrm{ppm}(\mathrm{d}, 2 \mathrm{H}, J=9.2 \mathrm{~Hz})$.

${ }^{13} \mathrm{C}$ NMR $(100 \mathrm{MHz})\left(\mathrm{CDCl}_{3}\right): \delta=14.29,22.83,26.03,28.99,29.36,29.41,31.96,69.02$, $98.96,113.75,125.78,126.71,127.38,128.18,128.25,129.08,134.39,134.81,138.05$, $149.77,156.10,158.69,160.78 \mathrm{ppm}$.

TOF MS ES+ $(\mathrm{M}+\mathrm{H})$ Calcd for $\mathrm{C}_{48} \mathrm{H}_{51} \mathrm{~F}_{4} \mathrm{~N}_{4} \mathrm{O}_{2}:$ 791.3948, found 791.3824 . 
(S)-(-)-2,2'-bis((E)-(2,6-difluoro-4-((4-(4-pentylcyclohexyl)benzyl)oxy)phenyl)di-

azinyl)-1,1'-binaphthalene $1 \mathbf{b}$ : a mixture of the intermediate compound $(0.039 \mathrm{~g}, 0.068$ mmol), (4-(4-pentylcyclohexyl)phenyl)methanol $\quad(0.044 \quad \mathrm{~g}, \quad 0.170 \quad \mathrm{mmol}), \quad$ diethyl azodicarboxylate (DEAD) $(0.036 \mathrm{~g}, 0.204 \mathrm{mmol})$ and triphenyl phosphine $\left(\mathrm{PPh}_{3}\right)(0.054 \mathrm{~g}$, $0.204 \mathrm{mmol})$ in THF $(10 \mathrm{ml})$ is refluxed under $\mathrm{N}_{2}$ and stirred overnight. After cooling to room temperature, the resulting solution is diluted with ethyl acetate $(30 \mathrm{~mL})$ and then washed with water 3 times. The formed organic layer is collected, dried over $\mathrm{MgSO}_{4}$ and the solvent is evaporated under reduced pressure. The residue is purified by column chromatography on silica gel using the mixture of n-hexane and ethyl acetate (20:1) as an eluent to get the red solid $\mathbf{1 b}$ with $30 \%$ yields.

${ }^{1} \mathrm{H}$ NMR $(400 \mathrm{MHz})\left(\mathrm{CDCl}_{3}\right): \delta=0.90(\mathrm{t}, 6 \mathrm{H}, J=7.0 \mathrm{~Hz}), 1.03(\mathrm{~m}, 4 \mathrm{H}), 1.36-1.17(\mathrm{~m}$, 24H), $1.42(\mathrm{~m}, 4 \mathrm{H}), 1.86(\mathrm{dd}, \quad 8 \mathrm{H}, J=12.0,3.4 \mathrm{~Hz}), 2.46(\mathrm{tt}, 2 \mathrm{H}, J=12.2,3.1 \mathrm{~Hz}), 4.90(\mathrm{~s}$, 4H), $6.44-6.33(\mathrm{~m}, 4 \mathrm{H}), 7.31-7.16(\mathrm{~m}, 12 \mathrm{H}), 7.44(\mathrm{dd}, 2 \mathrm{H}, J=8.5,1.1 \mathrm{~Hz}), 7.50$ (ddd, $2 \mathrm{H}, J=8.1,6.8,1.2 \mathrm{~Hz}), 7.95(\mathrm{~d}, 2 \mathrm{H}, J=8.2 \mathrm{~Hz}), 8.01(\mathrm{~d}, 2 \mathrm{H}, J=8.2 \mathrm{~Hz}), 8.11 \mathrm{ppm}(\mathrm{d}, 2 \mathrm{H}$, $J=9.0 \mathrm{~Hz})$.

${ }^{13} \mathrm{C}$ NMR $(100 \mathrm{MHz})\left(\mathrm{CDCl}_{3}\right): \delta=14.16,22.75,26.67,29.74,32.23,33.57,34.29,37.29$, $37.39,44.41,70.64,99.21,99.47,113.52,125.84,125.93,126.54,127.24,127.68,127.98$, $128.07,128.90,132.70,134.15,134.63,137.92,148.36,149.56,155.82,155.89,158.41$, $158.49,160.12 \mathrm{ppm}$.

TOF MS ES+ $(\mathrm{M}+\mathrm{H})$ Calculated for $\mathrm{C}_{68} \mathrm{H}_{71} \mathrm{~F}_{4} \mathrm{~N}_{4} \mathrm{O}_{2}$ : 1051.5513, found 1051.4021 . 
Compound (S)-(-)-2,2'-bis((E)-(4-((4-(4-pentylcyclohexyl)benzyl)oxy)phenyl)diazenyl)-

$\mathbf{1 , 1}$ '-binaphthalene $\mathbf{2 b}$ is synthesized following the same procedure as for compound $\mathbf{1 b}$.

${ }^{1} \mathrm{H} \mathrm{NMR}(400 \mathrm{MHz})\left(\mathrm{CDCl}_{3}\right): \delta=0.90(\mathrm{t}, 6 \mathrm{H}, J=6.9 \mathrm{~Hz}), 1.04(\mathrm{~m}, 4 \mathrm{H}), 1.38-1.16(\mathrm{~m}, 3 \mathrm{H})$, $1.47-1.36(\mathrm{~m}, 4 \mathrm{H}), 1.92-1.81(\mathrm{~m}, 8 \mathrm{H}), 2.46(\mathrm{tt}, 2 \mathrm{H}, J=12.2,3.1 \mathrm{~Hz}), 4.96(\mathrm{~s}, 4 \mathrm{H}), 6.84-$ $6.76(\mathrm{~m}, 4 \mathrm{H}), 7.20(\mathrm{~d}, 4 \mathrm{H}, J=8.2 \mathrm{~Hz}), 7.36-7.23(\mathrm{~m}, 10 \mathrm{H}), 7.53-7.41(\mathrm{~m}, 4 \mathrm{H}), 8.02-$ $7.93(\mathrm{~m}, 2 \mathrm{H}), 8.15 \mathrm{ppm}(\mathrm{d}, 2 \mathrm{H}, J=8.9 \mathrm{~Hz})$.

${ }^{13} \mathrm{C}$ NMR $(100 \mathrm{MHz})\left(\mathrm{CDCl}_{3}\right): \delta=14.15,22.74,26.67,32.23,33.58,34.31,37.29,37.38$, $44.39,70.08,114.44,114.75,124.59,126.56,126.92,127.11,127.63,127.81,128.04,128.98$, $133.74,134.20,134.26,147.38,147.96,148.32,160.89$ ppm.

TOF MS ES+ $(\mathrm{M}+\mathrm{H})$ Calculated for $\mathrm{C}_{68} \mathrm{H}_{75} \mathrm{~N}_{4} \mathrm{O}_{2}$ : 979.5890 , found 979.5272.

\subsubsection{Photo switching and thermal relaxation of 1 and 2 in solution}

a)
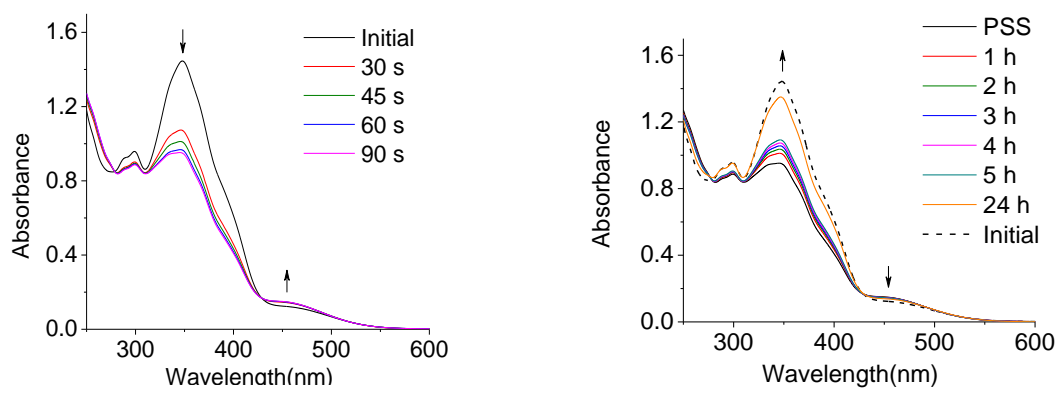
b)
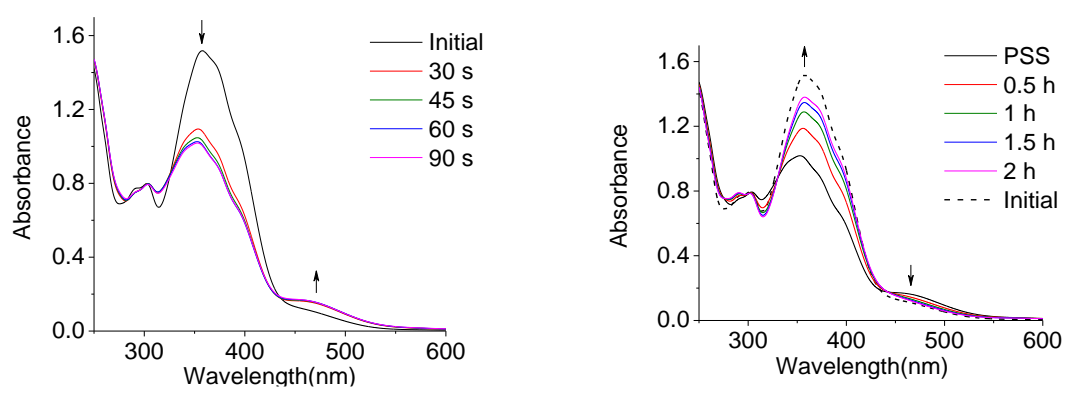

c)
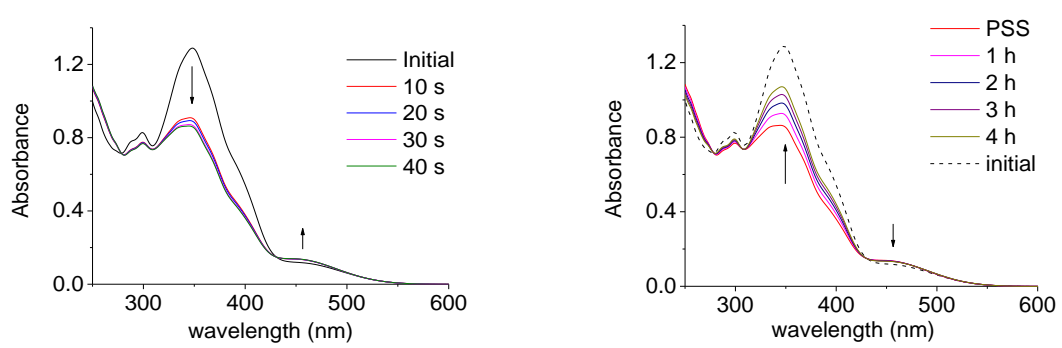

d)
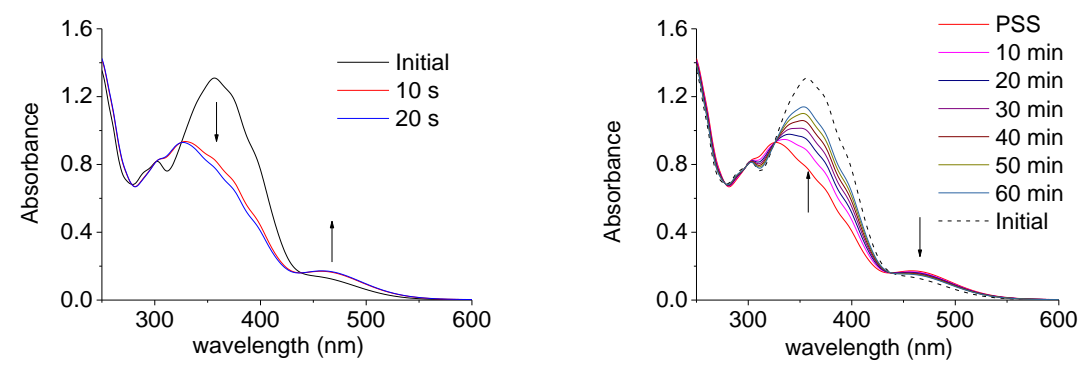

Figure S3.2. Changes in the UV-VIS absorption spectra of a) 1a, b) 2a, c) 1b, d) $2 \mathbf{b}$ in chloroform (c $=0.01 \mathrm{mM}$ ), during trans-to-cis isomerization (left column) and during the cis-to-trans thermal relaxation (right column). 


\subsubsection{Ratio of the cis-isomer of the dopants before UV exposure and at}

\section{the PSS}

1a. Initial trans $:$ cis $=98: 2$

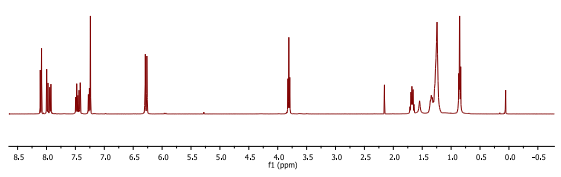

1a. PSS UV trans $:$ cis $=73: 27$

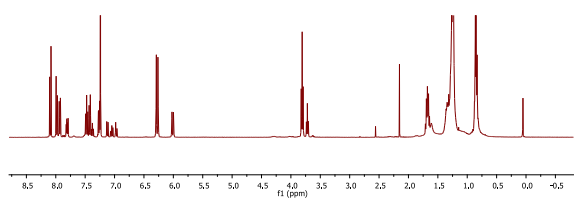

1b. Initial trans : cis $=100: 0$

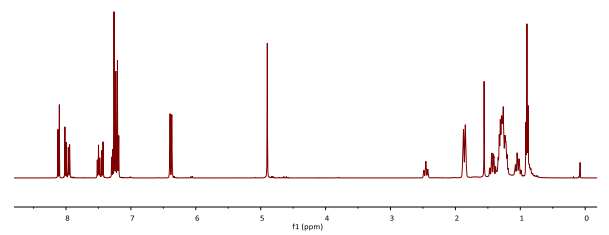

1b. PSS UV trans $:$ cis $=60: 40$

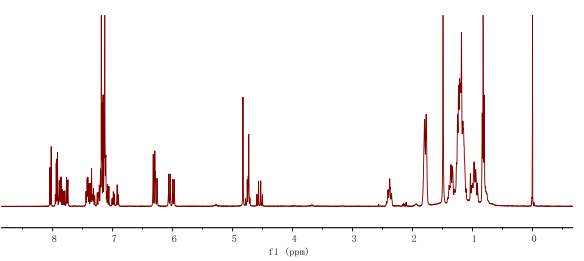

2a. Initial trans $:$ cis $=100: 0$

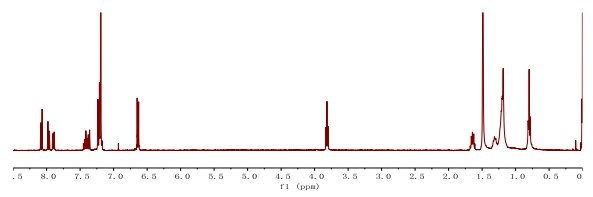

2a. PSS UV trans : cis $=70: 30$

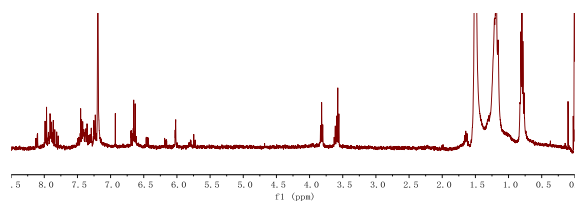

2b. Initial trans $:$ cis $=100: 0$

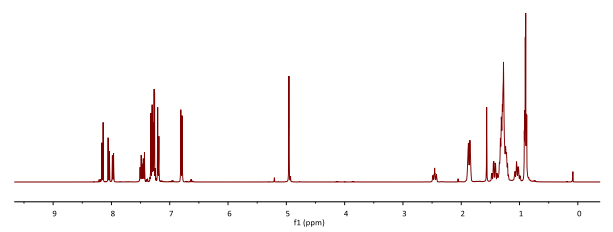

2b. PSS UV trans : cis $=62: 38$

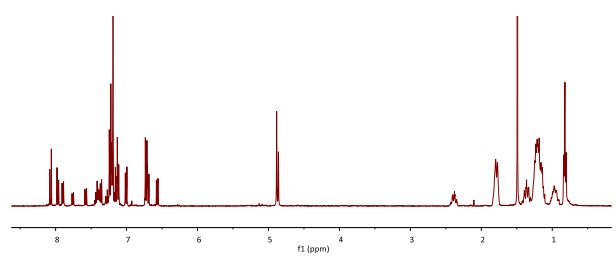

Figure S3.3. Estimation of the percentage of the cis-isomer before UV exposure and at the PSS for 1a, 1b (left column) and 2a $\mathbf{2 b}$ (right column) in chloroform solution. 


\subsubsection{Kinetics of the thermal relaxation in solution}

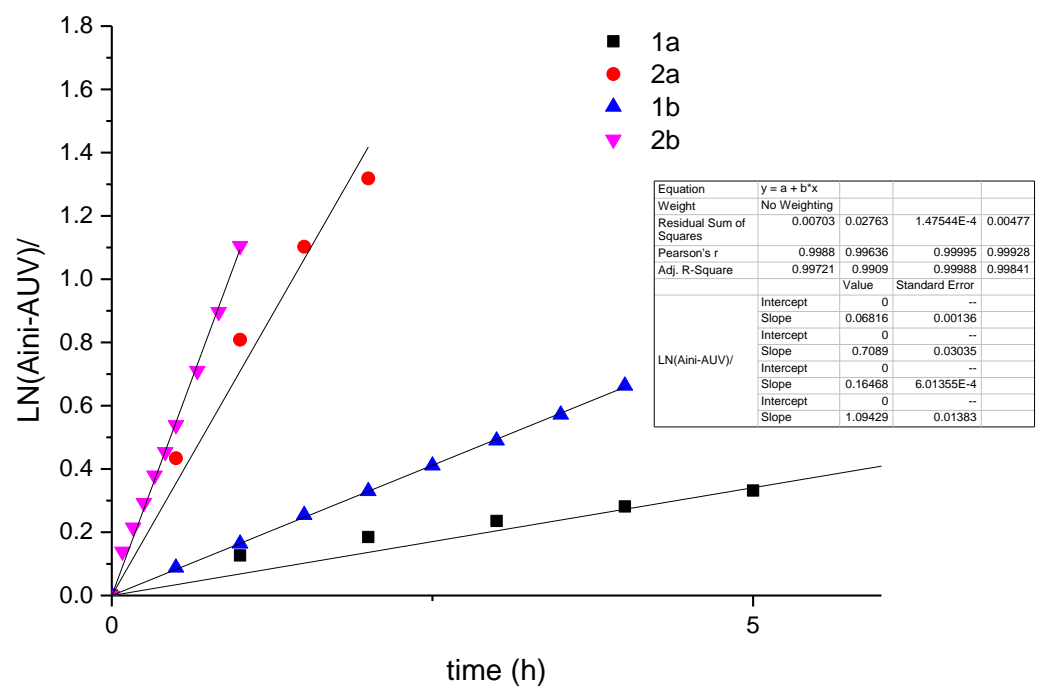

Figure S3.4. Kinetics of the thermal relaxation of cis-1a, cis-2a, cis-1b, cis-2b in solution.

\subsubsection{Photochemical behaviour of 1 and 2 in E7}

a)
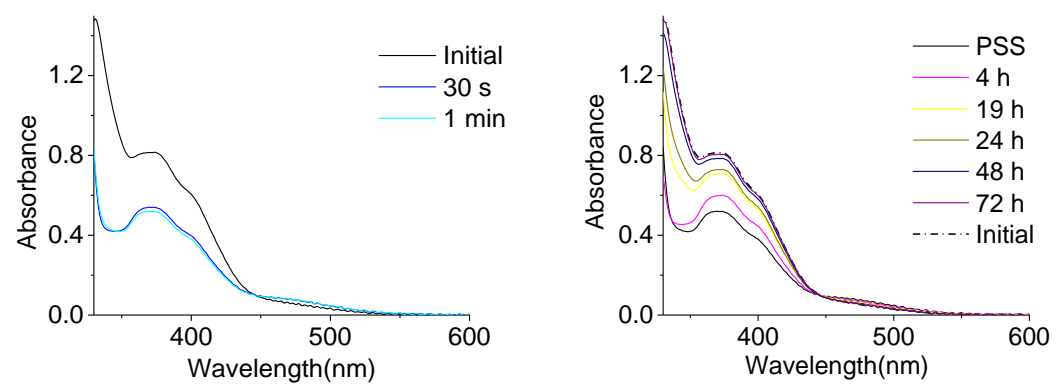
b)
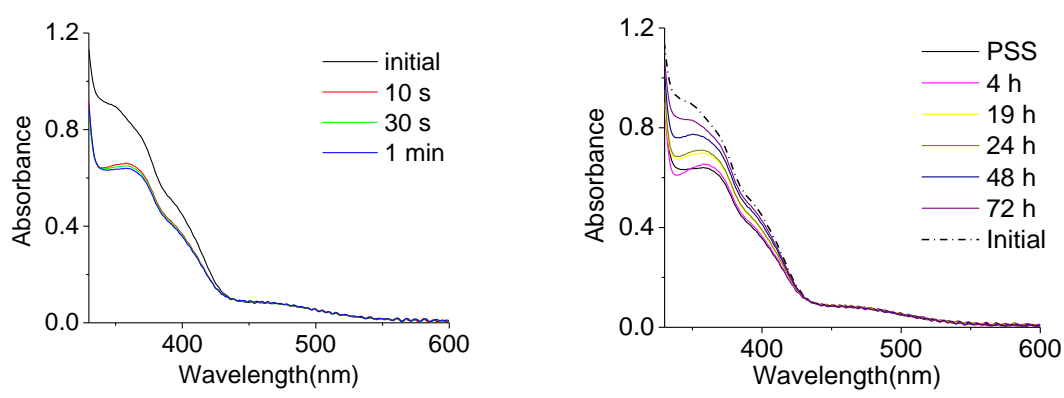

c)
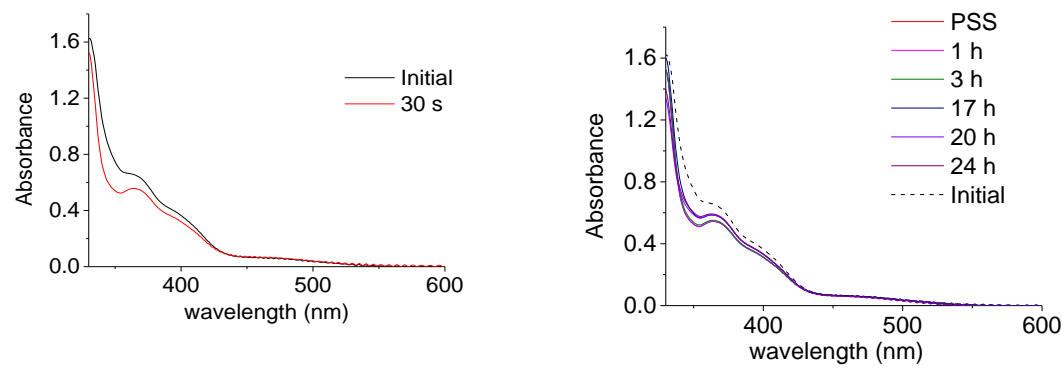

d)
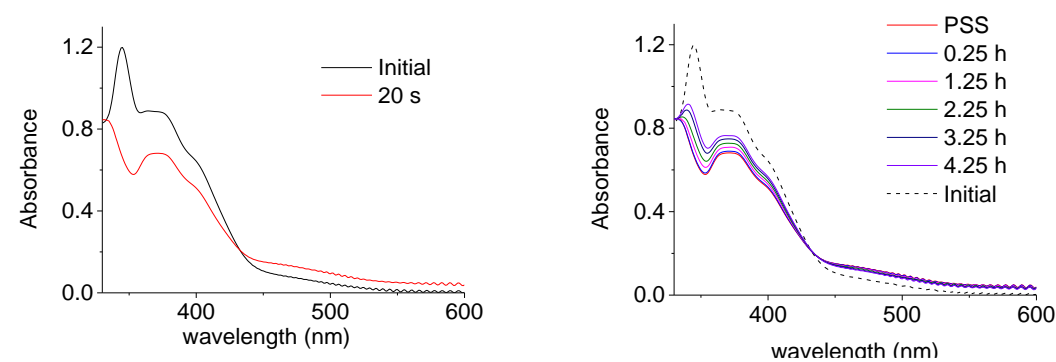

Figure S3.5. Changes in the UV-VIS absorption spectra of a) 1a, b) 2a, c) 1b, d) $2 \mathbf{b}$ in the liquid crystal E7, during trans-cis isomerization (left column) and during the thermal relaxation (right column). 


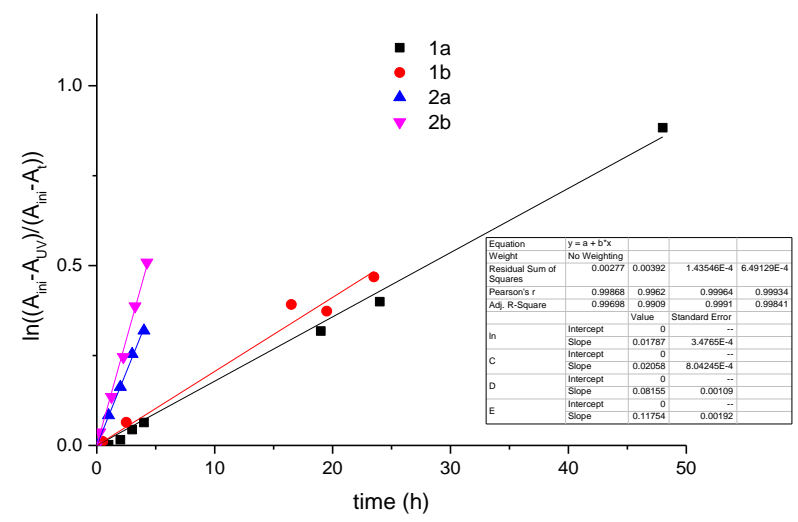

Figure S3.6. Kinetics of the thermal relaxation in E7 calculated by following changes in absorption.

\subsubsection{Photochemical behaviour and relaxation of 1 in MLC6608}

a)
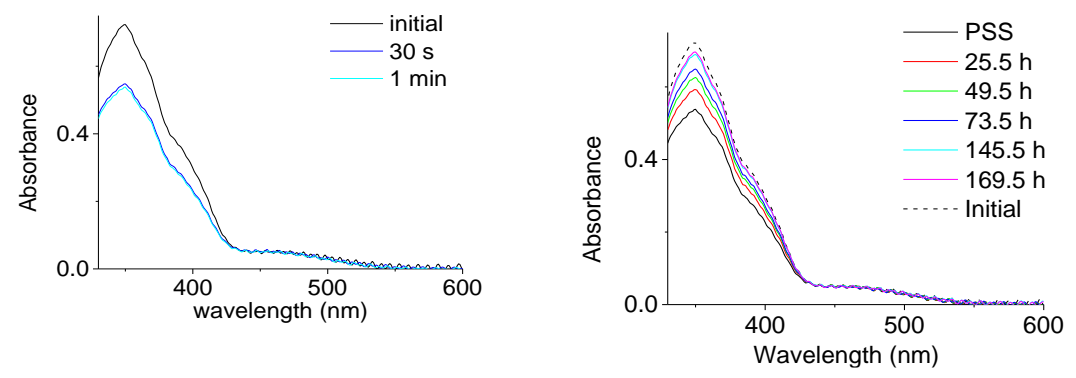

b)
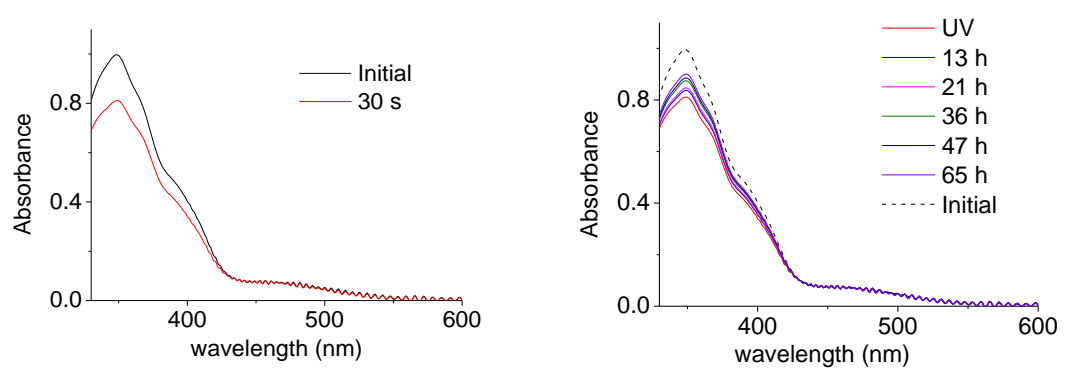

Figure S3.7. Changes in the UV-VIS absorption spectra of a) 1a, b) $\mathbf{1 b}$ in nematic LC host MLC 6608 at trans-cis isomerization (left column) and reversed thermal relaxation (right column). 


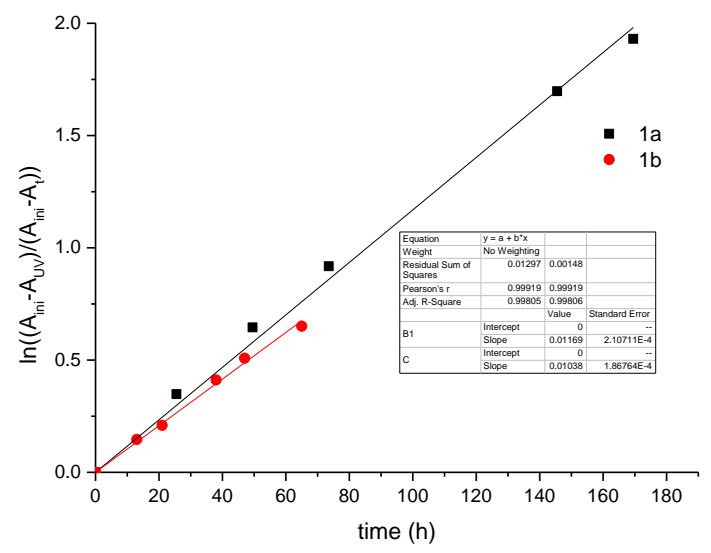

Figure S3.8. Kinetics of the thermal relaxation of $\mathbf{1 a}$ and $\mathbf{1 b}$ in MLC 6608 calculated by absorbance change.

\subsubsection{Helical twisting power (HTP) for 1 and 2 in E7}

a)
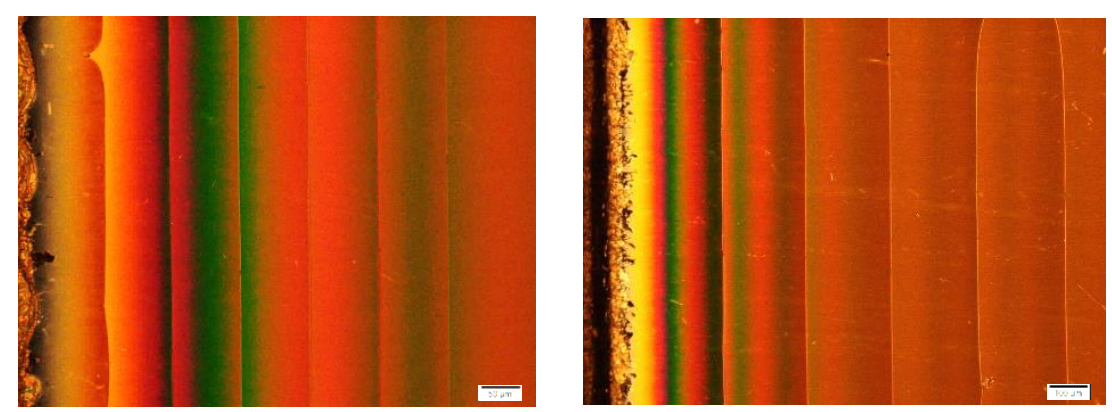

b)
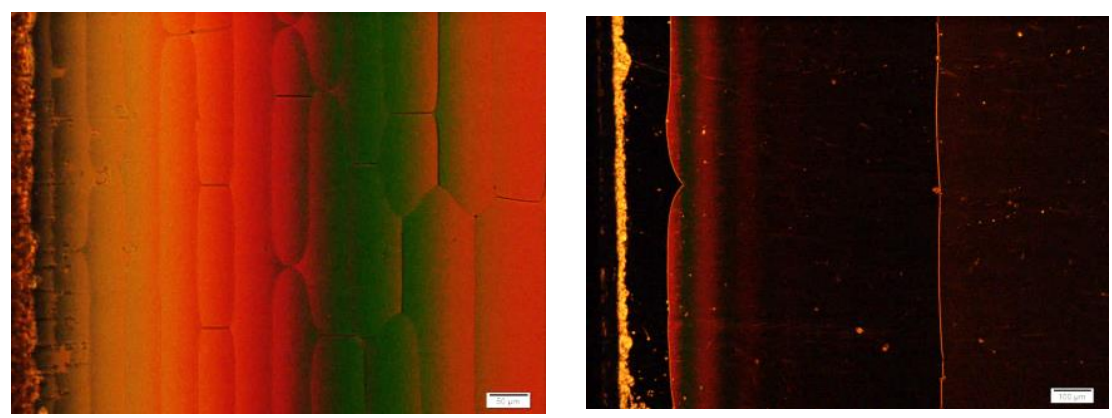
c)
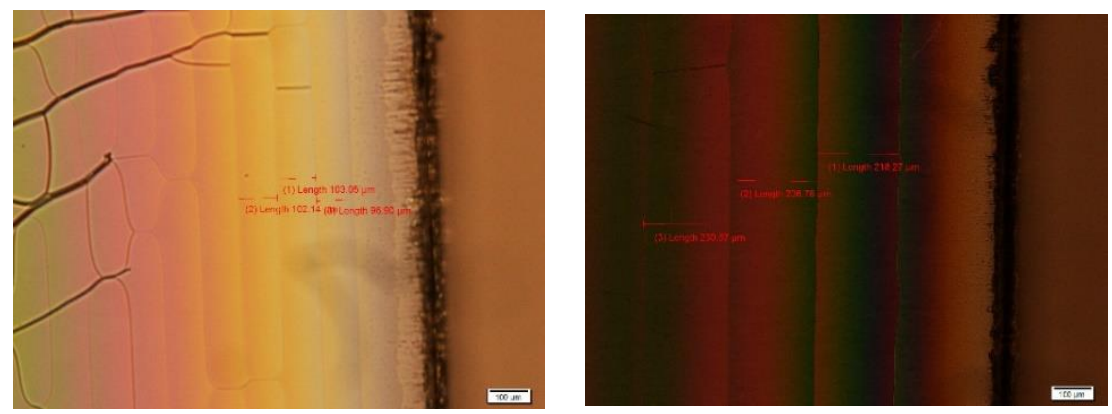

d)
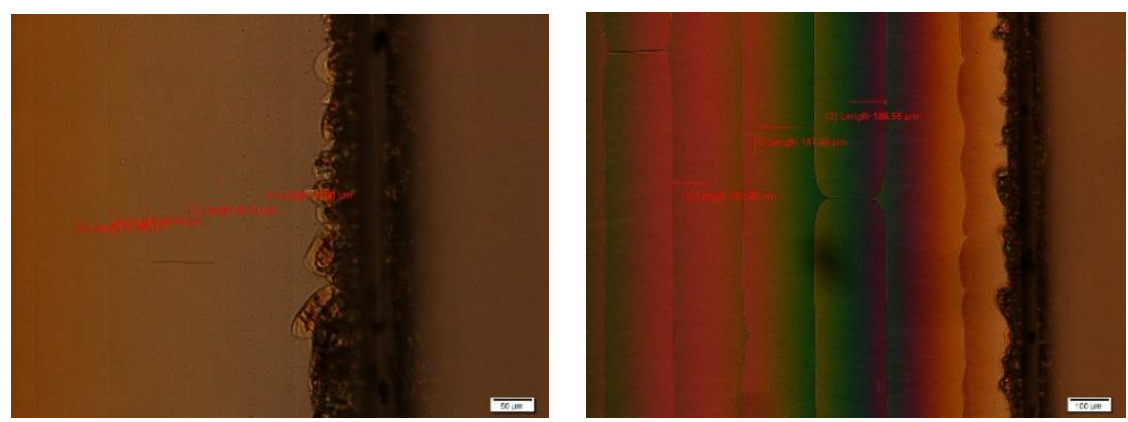

Figure S3.9. Grandjean-Cano textures induced by a) 1a, b) 2a, c) 1b, d) $2 \mathbf{b}$ in E7 at initial state (left column) and at the PSS (right column) (scale bar: 1a initial $=50 \mu \mathrm{m}, \mathbf{1} \mathbf{a}$ PSS $=100 \mu \mathrm{m}, \mathbf{2 a}$ initial $=50$ $\mu \mathrm{m}, \mathbf{2 a}$ PSS $=100 \mu \mathrm{m}, \mathbf{1 b}$ initial $=100 \mu \mathrm{m}, \mathbf{1 b}$ PSS $=100 \mu \mathrm{m}, \mathbf{2 b}$ initial $=50 \mu \mathrm{m}, \mathbf{2 b}$ PSS $=100 \mu \mathrm{m}$ ). 


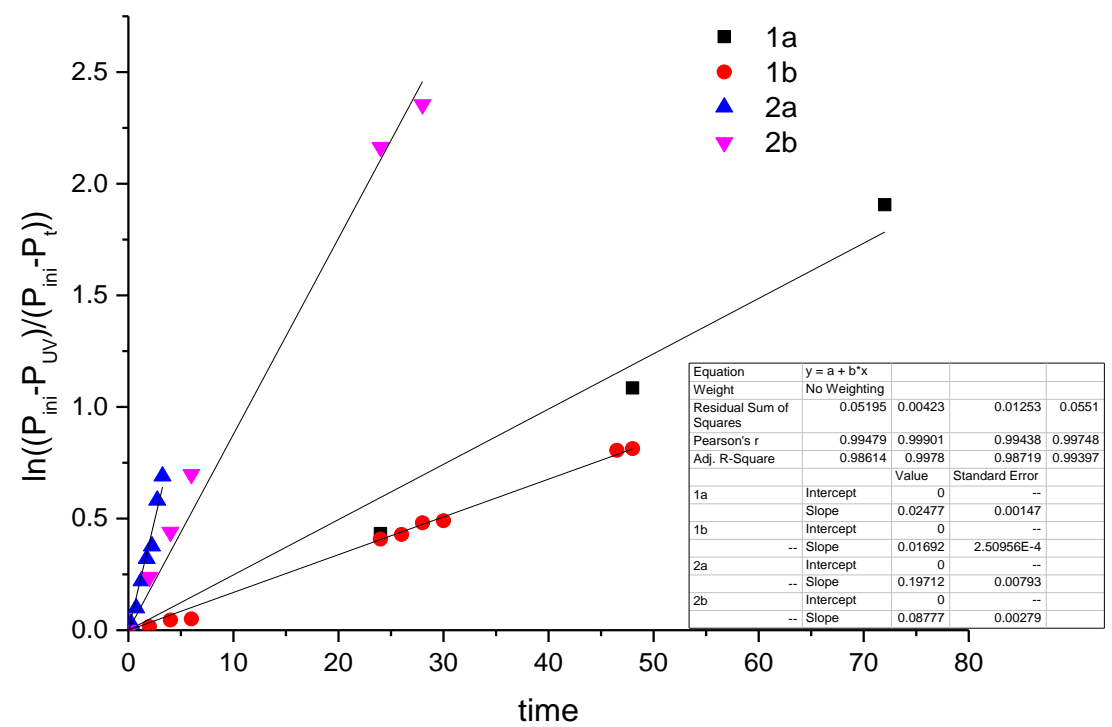

Figure S3.10. Kinetics of the thermal relaxation of cholesteric helices induced by illuminating 1a, 2a, $\mathbf{1 b}, \mathbf{2 b}$ in E7.

\subsubsection{Measurements of the helical twisting powers for 1 in MLC 6608}

a)
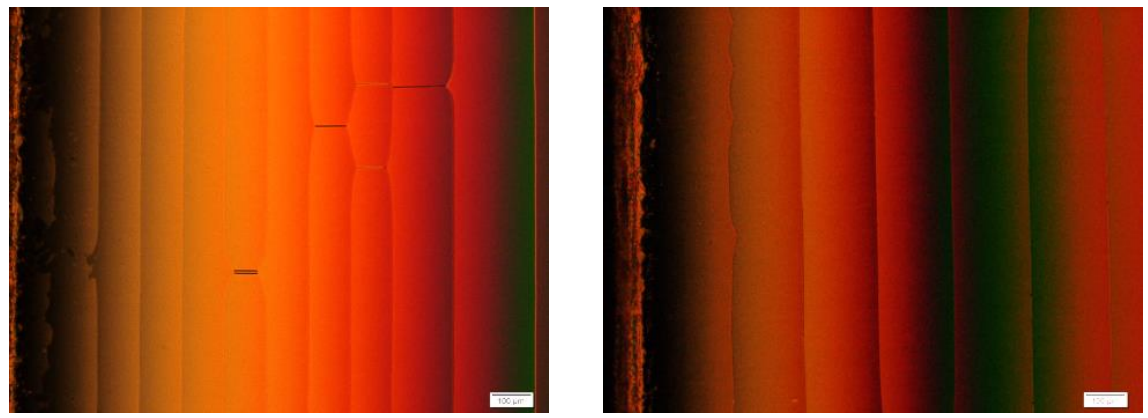
b)
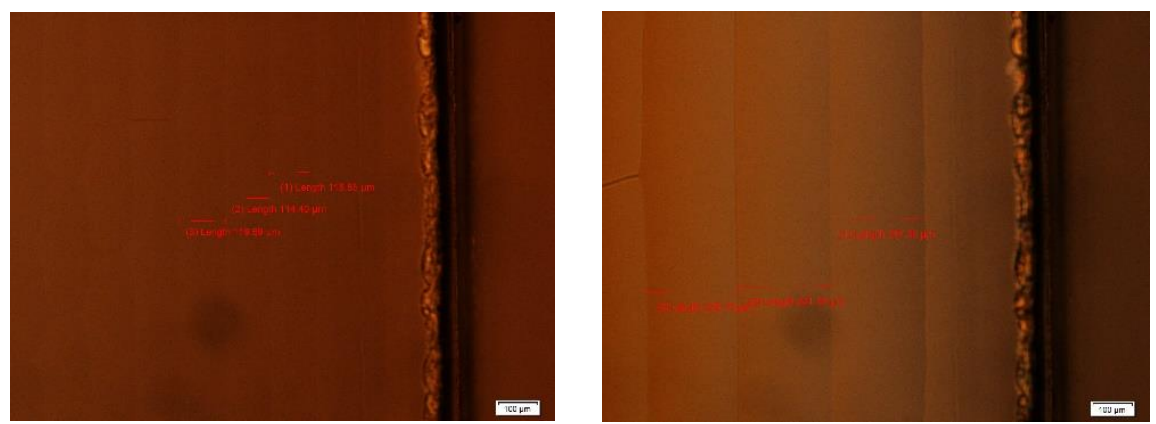

Figure S3.11. Grandjean-Cano textures induced by a) 1a, b) 1b in MLC6608 before UV exposure (left column) and at the PSS (right column). (all scale bars $=100 \mu \mathrm{m}$ ).

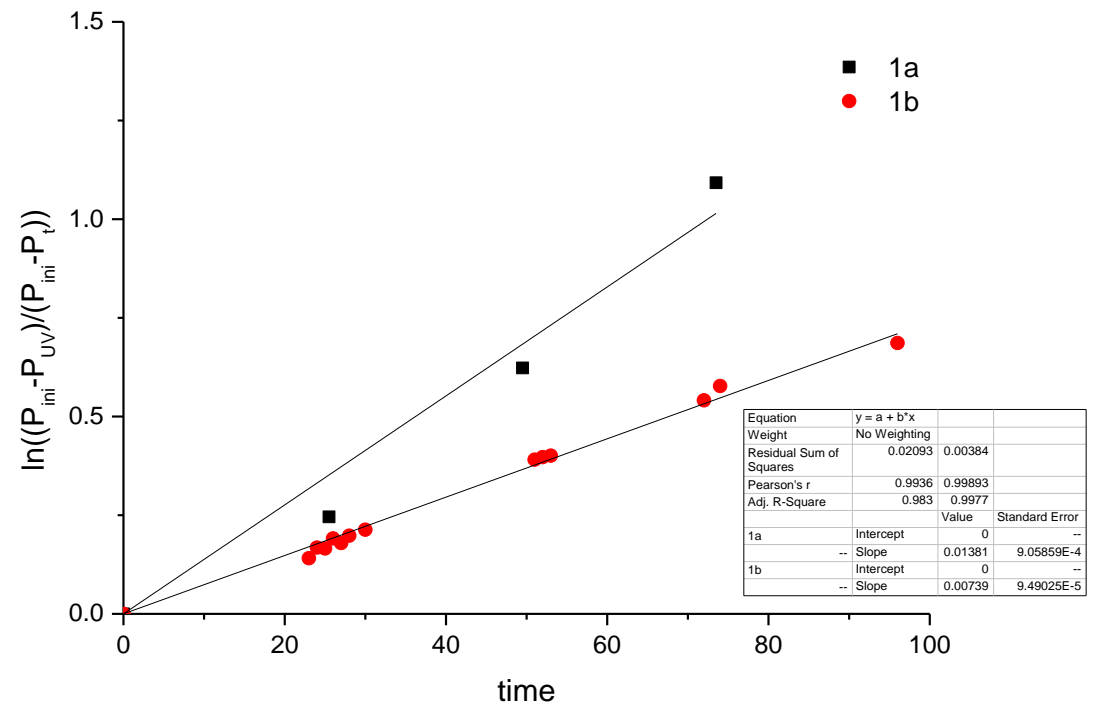

Figure S3.12. Kinetics of the thermal relaxation of cholesteric helices induced by illuminating 1a, 1b in MLC 6608 


\subsection{References}

[1] D. H. Qu, Q. C. Wang, Q. W. Zhang, X. Ma, H. Tian, Chem. Rev., 2015, 115, 7543.

[2] B. L. Feringa, W. R. Browne, Molecular Switches. Second edition. Wiley-VCH: Weinheim, 2011.

[3] W. R. Browne, B. L. Feringa, Nature Nanotech., 2006, 1, 25.

[4] R. Eelkema, Liq. Cryst., 2011, 38, 1641.

[5] R. Eelkema, M. M. Pollard, J. Vicario, N. Katsonis, B. S. Ramon, C. W. M. Bastiaansen, D. J. Broer, B. L. Feringa, Nature, 2006, 440, 163.

[6] S. M. Morrow, A. J. Bissette, S. P. Fletcher, Nature Nanotech., 2017, 12, 410.

[7] S. H. Chen, H. M. P. Chen, Y. Geng, S. D. Jacobs, K. L. Marshall, T. N. Blanton, Adv. Mater., 2003, 15, 1061.

[8] Z. Zheng, Y. Li, H. K. Bisoyi, L. Wang, T. J. Bunning, Q. Li, Nature, 2016, 541, 352.

[9] C. Loussert, S. Iamsaard, N. Katsonis, E. Brasselet. E., Adv. Mater., 2014, 26, 4242.

[10] T. Orlova, S. J. Aßhoff, T. Yamaguchi, N. Katsonis, E. Brasselet, Nat. Commun., 2015, 6,7603 .

[11] Y. Wang, Q. Li, Adv. Mater., 2012, 24, 1926.

[12] S. J. Aßhoff, S. Iamsaard, A. Bosco, J. J. L. M. Cornelissen, B. L. Feringa, N. Katsonis, Chem. Commun., 2013, 49, 4256.

[13] S. J. Aßhoff, S. Sukas, T. Yamaguchi, C. A. Hommersom, S. Le Gac, N. Katsonis, Sci. Rep., 2015, 5, 14183.

[14] R. Eelkema, R. A. van Delden, B. L. Feringa, Angew. Chem. Int. Ed., 2004, 43, 5013.

[15] T. J. White, D. J. Broer, Nature Mater., 2015, 14, 1087.

[16] S. Iamsaard, S. J. Aßhoff, B. Matt, T. Kudernac, J. J. L. M. Cornelissen, S. P. Fletcher, N. Katsonis, Nature Chem., 2014, 6, 229.

[17] S. J. Aßhoff, F. Lancia, S. Iamsaard, B. Matt, T. Kudernac, S. P. Fletcher, N. Katsonis, Angew. Chem. Int. Ed., 2017, 56, 3261.

[18] H. Traeubl, E. Sackmann, J. Am. Chem. Soc., 1972, 94, 4499.

[19] Q. Li, L. Green, N. Venkataraman, I. Shiyanovskaya, A. Khan, A. Urbas, J. W. Doane, J. Am. Chem. Soc., 2007, 129, 12908.

[20] J. Ma, Y. Li, T. White, A. Urbas, Q. Li, Chem. Commun., 2010, 46, 3463.

[21] L. Green, Y. Li, T. White, A. Urbas, T. Bunning, Q. Li. Org. Biomol. Chem., 2009, 7, 3930 .

[22] S. Pieraccini, S. Masiero, G. P. Spada, G. Gottarelli, Chem. Commun., 2003, 5, 598. 
[23] S. Pieraccini, G. Gottarelli, R. Labruto, S. Masiero, O. Pandoli, G. P. Spada, Chem. Eur. J., 2004, 10, 5632.

[24] R. A. Van Delden, T. Mecca, C. Rosini, B. L. Feringa, Chem. Eur. J., 2004, 10, 61.

[25] H. Krishna, Q. Li, Angew. Chem. Int. Ed., 2016, 55, 2994.

[26] D. Bléger, J. Schwarz, A. M. Brouwer, S. Hecht, J. Am. Chem. Soc., 2012, 134, 20597.

[27] C. Knie, M. Utecht, F. Zhao, H. Kulla, S. Kovalenko, A. M. Brouwer, P. Saalfrank, S. Hecht, D. Bléger, Chem. Eur. J., 2014, 20, 16492.

[28] J. Moreno, M. Gerecke, L. Grubert, S. A. Kovalenko, S. Hecht, Angew. Chem. Int. Ed., 2016, 55, 1544.

[29] S. Iamsaard, E. Anger, S. J. Aßhoff, A. Depauw, S. P. Fletcher, N. Katsonis, Angew. Chem. Int, Ed., 2016, 55, 9908.

[30] Q. Lim Y. Li, J. Ma, D. K. Yang, T. J. White, T. J. Bunning, Adv. Mater., 2011, 23, 5069.

[31] G. Heppke, F. Oestreicher, Mol. Cryst. Liq. Cryst., 1978, 41, 245.

[32] A. Bosco, M. G. M. Jongejan, R. Eelkema, N. Katsonis, E. Lacaze, A. Ferrarini, B. L. Feringa, J. Am. Chem. Soc., 2008, 130, 14615.

[33] O. S. Bushuyev, M. Aizawa, A. Shishido, C. J. Barrett, Macromol. Rapid Commun., 2017, 1700253. 


\section{Chapter 4}

\section{Competitive inclusion of molecular photo- switches in host cavities}

Azobenzene is a widely used guest molecule to build up dynamic photo-responsive hostguest systems. Here, we studied the host-guest complexation of a water-soluble, orthofluorinated azobenzene with two different host molecules, a cyclodextrin ( $\beta$-CD) and a cucurbit[8]uril (CB[8]), and demonstrate that in the presence of both, the guest forms two stable supramolecular complexes selectively. In its trans-form, it favors binding to $\beta$-CD, whereas in its cis-form it favors complexation to CB[8]. Upon light-triggered photoisomerization, the photoswitch was reversibly and selectively transferred from one host cavity to the other. This light-driven host exchange provides a new tool for supramolecular systems.

This chapter is published in H. Huang, A. Juan, N. Katsonis, J. Huskens, Tetrahedron, 2017, $73,4913$. 


\subsection{Introduction}

Host-guest chemistry is a useful tool to achieve functional architectures through a bottomup approach by joining molecular building blocks by non-covalent interactions in a controlled manner ${ }^{[1-4]}$. Incorporating stimuli-responsive building blocks in these architectures arguably constitutes the most straightforward strategy to endow these supramolecular assemblies with dynamic functions ${ }^{[5,6]}$. We focus on using light as a stimulus, which is a clean energy source and leads to photo-isomerization of photochromic molecular switches without further chemical waste ${ }^{[7]}$. Among other photo-responsive molecular switches $^{[8-10]}$, azobenzene derivatives have attracted special attention because their photoisomerization brings changes both in their conformation and in their dipolar moment ${ }^{[11,12]}$.

Macrocyclic molecules with convergent binding sites are extensively employed as molecular host cavities ${ }^{[13]}$. Typically, cyclodextrins are known to recognize and bind hydrophobic molecules as guests ${ }^{[2,14-16]}$. Host-guest interaction between azobenzenes and a $\beta$-cyclodextrin ( $\beta-C D)$ was first reported in $1980^{[17]}$ : the trans-form of the azobenzene was encapsulated into the hydrophobic cavity of $\beta-\mathrm{CD}$, and the trans-to cis- photo-isomerization led to the disassembly of the host-guest complex ${ }^{[12,18-21]}$. Cucurbit[n]urils (CB[n]) constitute another class of macrocyclic hosts. Due to the strong charge-dipole and H-bonding interactions, as well as the hydrophobic effect derived from the negative portals and rigid cavity, respectively, they form stable host-guest complexes with binding constants ranging up to $\sim 10^{17} \mathrm{M}^{-1}$ with cationic or electro-positive guest molecules ${ }^{[21-25]}$. A photo-responsive complex can be formed between $\mathrm{CB}[8]$ and azobenzene photo-switches, on the condition that a methyl viologen (MV) is present that mediates the formation of a ternary complex ${ }^{[6,26-28]}$. 
A major drawback of azobenzene switches, however, lies in their lack of thermal stability. Typically, the thermal relaxation of the unstable cis-isomer will drive any host-guest system back to equilibrium and results in the loss of UV input information. To maintain the cis-form, a continuous energy input is required ${ }^{[29]}$. For example, in the ternary complex (transAzo $\cdot \mathrm{MV}) \subset \mathrm{CB}[8]$, the UV irradiation led to disassembly of the complex. Once the input of UV light was removed, the cis-azobenzene relaxed back to the trans-form in several hours, by which the supramolecular system reverted to the state before the UV irradiation ${ }^{[6]}$.

a)
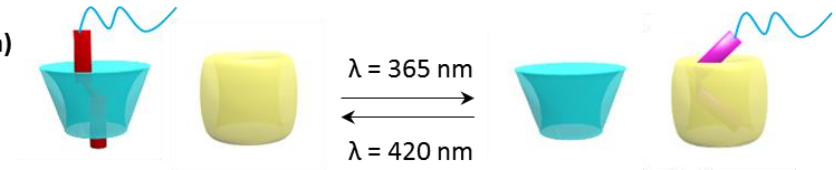

b)
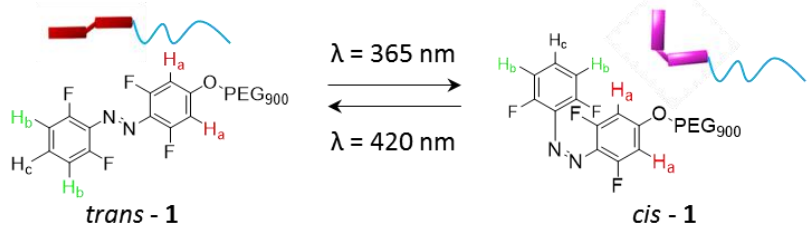

c)

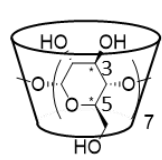

d)

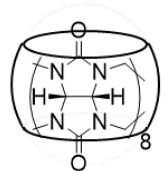

cis - 1

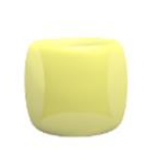

Scheme 4.1. a) Principle of using light to drive the selective inclusion of a molecular photo-switch in different molecular cavities. b) Molecular photo-switch 1 used in this study. c) $\beta$-Cyclodextrin ( $\beta$-CD) cavity used in this study. d) Cucurbit[8]uril (CB[8]) cavity used in this study.

Here, we demonstrate the design and synthesis of a dynamic, supramolecular host-guest system, which is stable in both "on" and "off" states, in order to provide two supramolecular states. We have designed and synthesized an ortho-fluorinated azobenzene (1) as building block, as ortho-fluorination is known to stabilize the cis-isomer of the azobenzene considerably $^{[30,31]}$, and report its dynamic complexation with two different host molecules, a 
cyclodextrin and a cucurbituril. We demonstrate that $\mathbf{1}$ can be reversibly translocated from one cavity to the other selectively upon illumination [Scheme 4.1].

\subsection{Results and discussion}

A neutral, water-soluble and thermally stable azobenzene switch $\mathbf{1}$ was designed and synthesized to mediate the dynamic supramolecular complexation [Scheme S4.1, S4.2] Directly after synthesis, the ratio between trans-1 and cis-1 was trans : cis $=94: 6$. Upon irradiation by UV light $(\lambda=365 \mathrm{~nm})$, the photo-stationary state was achieved yielding a ratio of trans : cis $=10: 90$. Upon irradiation with blue light $(\lambda=420 \mathrm{~nm})$ the back-isomerization to the trans form resulted in a photo-stationary state that was reached after 20 min irradiation, yielding a ratio of trans : cis $=57: 43$ [Figure $\mathbf{S 4 . 1}$.

The inclusion of an acid-functionalized fluorinated azobenzene into $\beta$-CD has been reported recently ${ }^{[32]}$. Interestingly, both the trans and cis-isomers could form supramolecular complexes with $\beta-\mathrm{CD}$, and the binding affinity of the $c i s$-isomer was even higher than that of the trans-isomer. In contrast, for non-fluorinated, traditional azobenzenes, the selectivity is in favor of binding of the trans-isomer ${ }^{[1,17,18]}$. The earlier reported fluorinated azobenzene is symmetric ${ }^{[32]}$, therefore we compared its supramolecular binding to $\beta-\mathrm{CD}$ to the behaviour of switch 1, which is non-symmetric. We started by investigating the complexation between molecule 1 and $\beta$-CD by ${ }^{1} \mathrm{H}$ NMR (in $\mathrm{D}_{2} \mathrm{O}$ ) and isothermal titration calorimetry (ITC, in $\mathrm{H}_{2} \mathrm{O}$ ). Because of the absence of protonatable sites in the guest and hosts, the $\mathrm{pH} / \mathrm{pD}$ was not controlled, nor were differences in $\mathrm{pH} / \mathrm{pD}$ or the affinity constants considered here. ITC 
indicates that cis-1 has a higher binding affinity to $\beta-\mathrm{CD}\left(\mathrm{K}=4.8 \times 10^{3} \mathrm{M}^{-1}\right)$ than trans-1 (K $=2.3 \times 10^{3} \mathrm{M}^{-1}$ ) $\left[\right.$ Table 4.1, Figure S4.2]. ${ }^{1} \mathrm{H}$ NMR titration experiments confirmed these results [Figure S4.3 - S4.6]. The orientation of the guest in the supramolecular complex was investigated by ${ }^{1} \mathrm{H}$ NMR Nuclear Overhausser Effect SpectroscopY (NOESY). The signal for $\mathrm{H}_{\mathrm{a}}$ on trans-1 showed a stronger correlation with $\mathrm{H}_{3}$ of $\beta-\mathrm{CD}$ than $\mathrm{H}_{5}$, while for $\mathrm{H}_{\mathrm{b}}$ only the correlation with $\mathrm{H}_{5}$ was observed [Figure S4.7]. Likewise, in the $c i s-1 \subset \beta-\mathrm{CD}$ complex, the correlation between $\mathrm{H}_{\mathrm{a}}$ and $\mathrm{H}_{3}$ was stronger than the $\mathrm{H}_{\mathrm{a}}-\mathrm{H}_{5}$ correlation, while $\mathrm{H}_{\mathrm{b}}$ was more correlated to $\mathrm{H}_{5}$ than to $\mathrm{H}_{3}$ [Figure $\mathbf{S 4 . 8}$ ]. The NOE effect thus indicates that, for both trans-1 $\subset \beta-C D$ and cis-1 $\subset \beta-C D$, the guest molecule is binding from the secondary face of the $\beta$-CD cavity. The difference between the binding affinities of the trans and cis complexes observed here is less than one order of magnitude. Therefore, upon illumination of such hostguest complexes, the photo-isomerization of the guest molecule cannot result in any significant changes in the distribution over the bound and unbound states. An additional component is thus required to release the azobenzene from the $\beta-\mathrm{CD}$ cavity, and thus to provide an "off" state to make the supramolecular system photo-switchable in its preference for different hosts.

Next, we studied the interaction between switch 1 and CB[8]. The first experiment was performed by ${ }^{1} \mathrm{H}$ NMR spectroscopy. In the 1:1 mixture of trans -1 and $\mathrm{CB}[8]$, the aromatic protons of 1 were almost not shifted [Figure S4.9], meanwhile in the sample with cis-1 and $\mathrm{CB}[8]$, the aromatic protons Ha were shifted from $\delta=6.68 \mathrm{ppm}$ to $\delta=6.28 \mathrm{ppm}$ [Figure S4.10]. These results reveal the existence of a supramolecular interaction between cis-1 and $\mathrm{CB}[8]$. This interaction was further investigated by titrating aqueous solutions of trans $\mathbf{- 1}$ and 
cis-1 $(\mathrm{c}=0.5 \mathrm{mM})$ into an aqueous $\mathrm{CB}[8]$ solution $(\mathrm{c}=0.05 \mathrm{mM})$, separately, monitored by ITC. The interaction between trans-1 and $\mathrm{CB}[8]$ was too weak to be detected by ITC, whereas cis-1 formed a cis-1 $\subset \mathrm{CB}[8]$ complex with a binding affinity of $\mathrm{K}=4.9 \times 10^{5} \mathrm{M}^{-1}[$ Figure S4.11].

Table 4.1. Binding affinities and thermodynamic parameters for the host-guest interaction of azobenzene 1 with different hosts.

$\begin{array}{ccccc}\text { Guest } & \text { Host } & \mathrm{K}\left(\mathrm{M}^{-1}\right) & \Delta \mathrm{H}\left(\mathrm{kcal} \mathrm{mol}^{-1}\right) & \mathrm{T} \Delta \mathrm{S}\left(\mathrm{kcal} \mathrm{mol}^{-1}\right) \\ \text { trans-1 } & \beta-\mathrm{CD} & 2.3 \times 10^{3} & -2.1 & 2.5 \\ \text { cis-1 } & \beta-\mathrm{CD} & 4.8 \times 10^{3} & -9.2 & -4.2 \\ \text { trans-1 } & \mathrm{CB}[8] & - & - & - \\ \text { cis-1 } & \mathrm{CB}[8] & 4.9 \times 10^{5} & -9.6 & -1.8\end{array}$

The thermodynamic values associated with the complexation of 1 into the $\beta-C D$ and $C B[8]$ cavities are summarized in Table 4.1. Overall, they indicate that $\mathbf{1}$ forms host-guest complexes with $\beta-\mathrm{CD}$ in both the trans and the cis-form, the binding affinity of the cis-form complex being higher than that of the trans-form. In contrast, while no inclusion occurs between trans-1 and $\mathrm{CB}[8]$, cis-1 forms a supramolecular complex with $\mathrm{CB}[8]$, with a binding constant that is two orders of magnitude higher than that of $\beta-\mathrm{CD}$. This behaviour contrasts with the dynamic complexation of regular azobenzene, which is included into $\beta$ CD only in its trans-form and is released from the cavity upon UV irradiation, while no stable complex is formed with $\mathrm{CB}[8]$, neither in the trans nor in the cis-form ${ }^{[26-28]}$. Moreover, trans$\mathbf{1}$ shows a positive entropy change upon complexation with $\beta-\mathrm{CD}$, and the absolute value of this entropic change is higher than the enthalpic change [Table 4.1]. These values indicate 
that, in the complex, there is a relatively high conformational and transitional flexibility, and the main factor leading to the complexation is the hydrophobic interaction between the fluorinated azobenzene moiety and the cavity. Meanwhile, the inclusion of cis-1 into $\beta-\mathrm{CD}$ is an enthalpy-driven process associated with a considerable entropy penalty, which indicates that van der Waals interactions between the guest and the cavity resulting in a tight fit constitute the main driving force of the $c i s-1 \subset \beta-\mathrm{CD}$ complexation ${ }^{[33]}$. In the $\mathrm{CB}[8]$ complex, additional electrostatic interactions occur between host and guest, besides the van der Waals and hydrophobic interactions, which result here in an approximately two orders of magnitude higher binding affinity than those observed for the $\beta-\mathrm{CD}$ complexes ${ }^{[22]}$. Overall, considering that the difference between the binding affinity of cis-1 $\subset \mathrm{CB}[8]$ is higher than either trans$\mathbf{1} \subset \beta$-CD or $c i s-1 \subset \beta$-CD by two orders of magnitude, these thermodynamic values support the possibility of a light-controlled, reversible, and competitive binding of the guest between the two different host molecules.

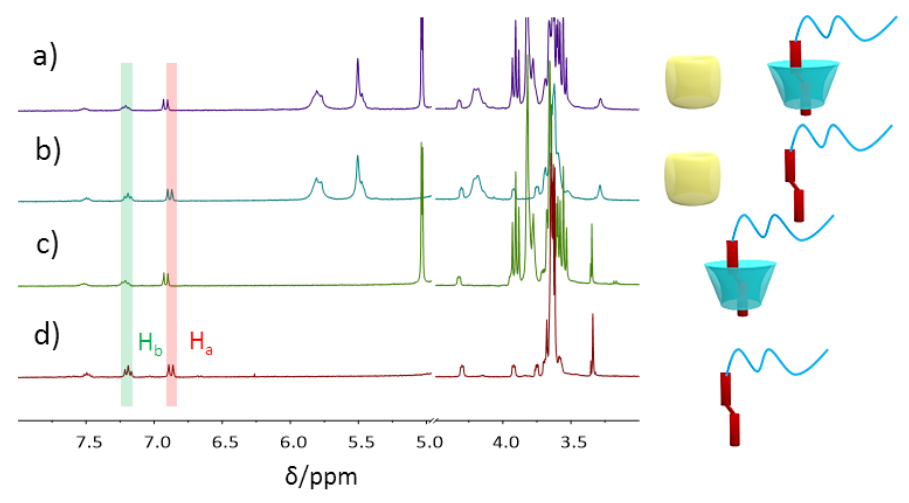

Figure 4.1. Comparison of ${ }^{1} \mathrm{H}$ NMR spectra of a) trans-1 with $\beta$-CD and $\mathrm{CB}[8]$ (1:1:1 ratio), b) trans$\mathbf{1}$ with $\mathrm{CB}[8](1: 1)$, c) trans-1 with $\beta-\mathrm{CD}(1: 1)$, d) trans-1. The concentration of $\mathbf{1}$ was in all cases $0.5 \mathrm{mM}$.

The competitive binding behaviour was investigated by ${ }^{1} \mathrm{H}$ NMR in a three-component 
system comprising $1: \beta-C D: C B[8]=1: 1: 1\left(c=0.5 \mathrm{mM}^{2} \mathrm{D}_{2} \mathrm{O}\right)$. We note that this concentration is above the solubility limit $(0.05 \mathrm{mM})$ of $\mathrm{CB}[8]$, so that when $\mathrm{CB}[8]$ is not complexed to the guest, part of the host is dispersed in the sample without complete dissolution. The proton Ha was monitored and compared to the samples of pure $\mathbf{1}$ and of $\mathbf{1}$ with the different host molecules separately. For the pure trans-1, the $\mathrm{H}_{\mathrm{a}}$ signal was found at $\delta=6.89 \mathrm{ppm}$, and this signal was not shifted in the mixture of trans-1 and CB[8]. In the sample of trans $\mathbf{1}$ and $\beta-\mathrm{CD}$, the proton $\mathrm{H}_{\mathrm{a}}$ was shifted to $\delta=6.93 \mathrm{ppm}$. In the threecomponent sample, the signal of $\mathrm{H}_{\mathrm{a}}$ was found at $\delta=6.93 \mathrm{ppm}$, which confirmed the

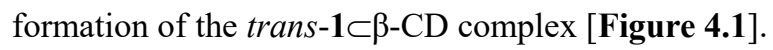

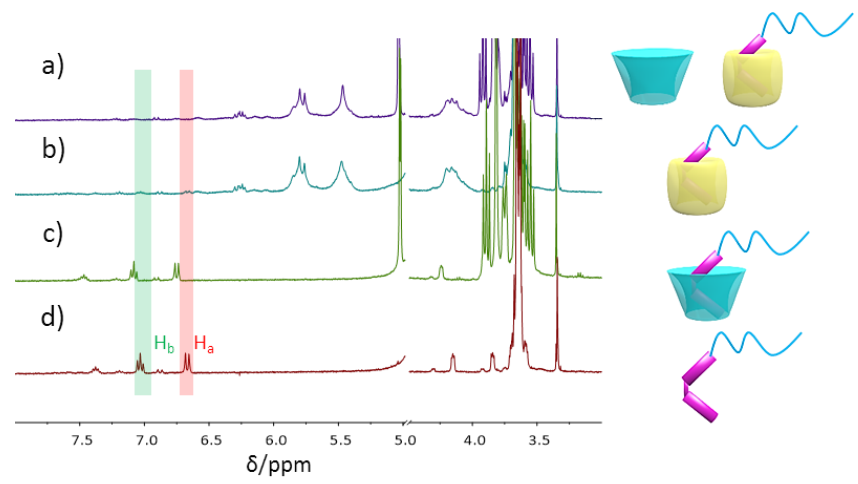

Figure 4.2. Comparison of ${ }^{1} \mathrm{H}$ NMR spectra of a) cis-1 with $\beta-\mathrm{CD}$ and $\mathrm{CB}[8](1: 1: 1)$, b) cis-1 with $\mathrm{CB}[8](1: 1), \mathrm{c})$ cis-1 with $\beta$-CD $(1: 1)$, d) cis-1. The concentration of $\mathbf{1}$ was in all cases $0.5 \mathrm{mM}$.

When cis-1 was used as obtained upon UV irradiation, the $\mathrm{H}_{\mathrm{a}}$ signal of proton of the cis-

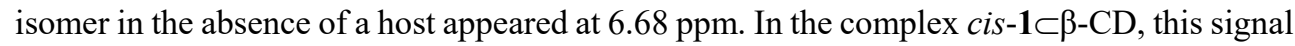
was shifted to $\delta=6.74 \mathrm{ppm}$, while in the complex cis $-1 \subset \mathrm{CB}[8]$, this signal was observed to $\delta=6.28 \mathrm{ppm}$. When both $\beta-\mathrm{CD}$ and $\mathrm{CB}[8]$ were present, the signal of proton $\mathrm{H}_{\mathrm{a}}$ was found at $\delta=6.28 \mathrm{ppm}$, which confirmed that the $c i s-1 \subset \mathrm{CB}[8]$ complex had formed selectively [Figure 4.2], in agreement with the measured differences in binding affinity [Table 4.1]. We 
note that apparently the binding affinity of $c i s-\mathbf{1}$ for $\mathrm{CB}[8]$ is sufficiently strong to pull all undissolved host into solution, even in the presence of well-dissolved and competing $\beta$-CD.

To study the photo-responsiveness of the competitive complexation in situ, we prepared a three-component sample consisting of $\mathbf{1}, \beta-\mathrm{CD}$, and $\mathrm{CB}[8]$, which was irradiated while measuring $\mathrm{UV} / \mathrm{Vis}$ absorption and ${ }^{1} \mathrm{H}$ NMR spectra. We chose a ratio of $\mathbf{1}: \beta-\mathrm{CD}: \mathrm{CB}[8]=$ $1: 10: 1(\mathrm{c}=0.05 \mathrm{mM})$ to stay below the solubility limit of $\mathrm{CB}[8]$, but at the same time have a sufficiently high $\beta-\mathrm{CD}$ concentration to still observe binding of the guest. The experiment was started from the trans-form as the initial state. In the UV/Vis absorption spectra, for trans -1 , two major absorbance peaks are present at $\lambda=335 \mathrm{~nm}$ and $\lambda=428 \mathrm{~nm}$, which are associated to $\pi-\pi^{*}$ and $n-\pi^{*}$ transitions, respectively [Figure 4.3]. Upon irradiation with UV light $(\lambda=365 \mathrm{~nm})$, the decrease of the $\pi-\pi^{*}$ absorbance confirms that trans-1 isomerizes to the $c i s$-form. Moreover, the $n-\pi^{*}$ absorbance decreased as well, and the associated peak blueshifted to $\lambda=418 \mathrm{~nm}$. When the $c i s$-isomer mixture was irradiated with blue light, the UV/vis spectrum indicated cis-to-trans conversion. The incomplete cis-to-trans conversion, which is in agreement with ${ }^{1} \mathrm{H}$ NMR experiments [Figure 4.5], is attributed to the minor change of the $\mathrm{n}-\pi^{*}$ absorbance upon switching. The $c i s-1 \subset \mathrm{CB}[8]$ complex was kept in the dark for up to two weeks, after which the UV/Vis absorption spectrum did not show any significant change, thus confirming that $c i s-\mathbf{1}$ is thermally stable. 
a)
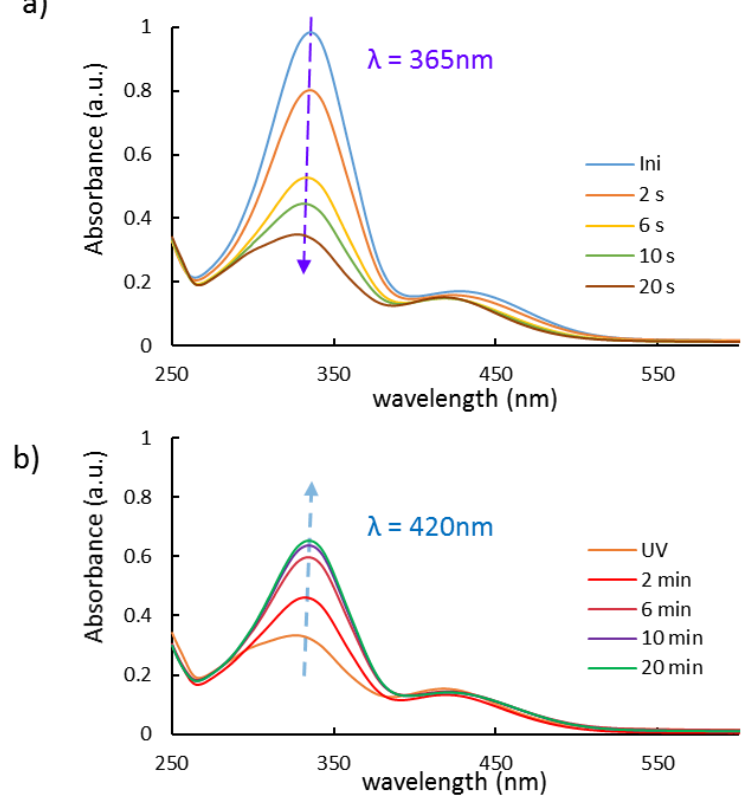

Figure 4.3. UV/Vis absorption spectra of $1, \beta-C D$ and $C B[8](1: 10: 1$ in water solution, $c=0.05 \mathrm{mM})$ for the a) trans-to-cis and b) cis-to-trans conversions as induced by illumination with $\lambda=365 \mathrm{~nm}$ and $\lambda=420 \mathrm{~nm}$ light, respectively.

In the ${ }^{1} \mathrm{H}$ NMR experiments, initially, the signal of $\mathrm{H}_{\mathrm{a}}$ was found at $\delta=6.93 \mathrm{ppm}$, which suggested that the trans-1 was bound to $\beta-C D$. Following irradiation with UV light $(\lambda=365$ $\mathrm{nm}$ ), this signal decreased and eventually disappeared entirely after $10 \mathrm{~min}$, while another group of signal s appeared at $\delta=6.28 \mathrm{ppm}$. These results suggested that during the UV irradiation, trans $\mathbf{- 1}$ was converted into $c i s-\mathbf{1}$ and was consequently transferred from the $\beta$ $\mathrm{CD}$ cavity to the $\mathrm{CB}[8]$ cavity. Even though the $\beta-\mathrm{CD}$ concentration was ten times higher than that of $\mathrm{CB}[8]$, cis-1 still preferred to bind to the $\mathrm{CB}[8]$ cavity. Subsequently, the cis-totrans isomerization was performed with blue light illumination $(\lambda=420 \mathrm{~nm})$. Under illumination, the signal at $\delta=6.28 \mathrm{ppm}$ decreased and the signal at $\delta=6.93 \mathrm{ppm}$ re-appeared, which indicates that cis-1 was converted into trans-1 and concomitantly transferred back 
from the $\mathrm{CB}[8]$ cavity to the $\beta$ - $\mathrm{CD}$ cavity. After 30 min the photo-stationary state was reached with a trans : cis ratio of $57: 43$, which is in agreement with UV/Vis absorption experiments [Figure 4.4]. The rate of photo-isomerization of cis-to-trans appeared to be on the order of $0.14 \mathrm{~min}^{-1}$, both in the presence [Figure 4.4] and absence [Figure S4.12] of CB[8], which indicates that the complexation of cis-1 by $\mathrm{CB}[8]$ did not affect the rate of photoisomerization.
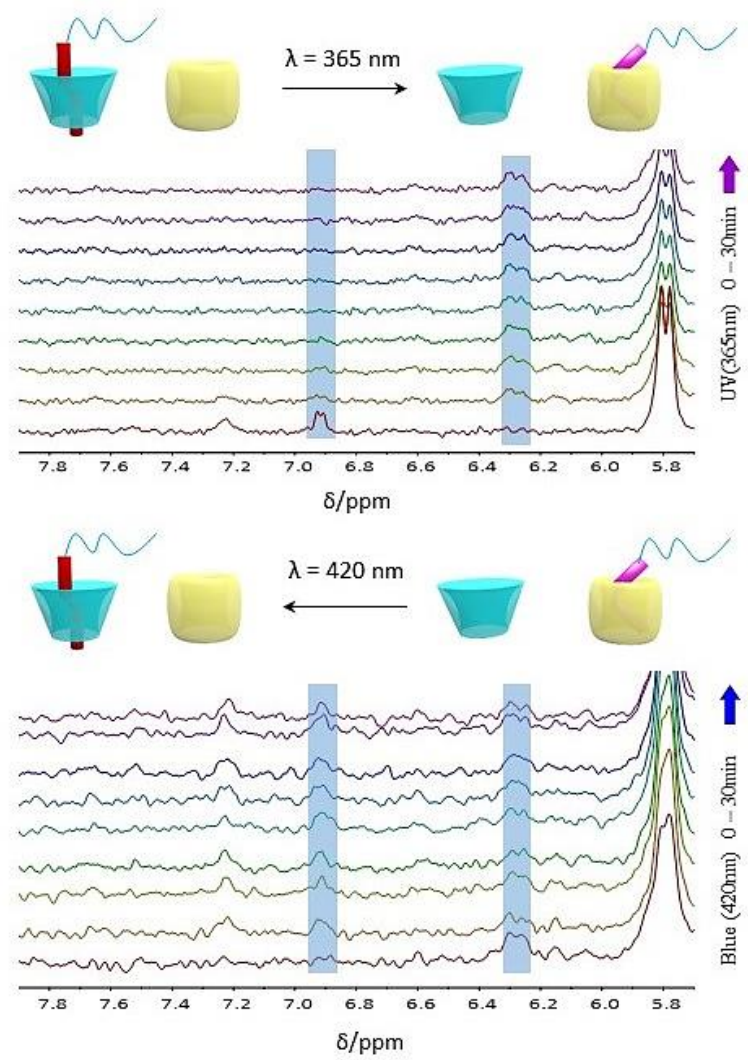

Figure 4.4. ${ }^{1} \mathrm{H}$ NMR spectra of the in-situ switching experiments of $1: \beta-\mathrm{CD}: \mathrm{CB}[8]=1: 10: 1$ ( $\mathrm{c}=0.05 \mathrm{mM}$ ), showing (from bottom to top for 0-30 min of illumination) the a) trans-to-cis and b) cisto-trans conversions as induced by illumination with $\lambda=365 \mathrm{~nm}$ and $\lambda=420 \mathrm{~nm}$ light, respectively. 


\subsection{Conclusions}

We have shown the dynamic complexation of the water-soluble and thermally stable molecular photo-switch 1 with two different host molecules, $\beta$ - $\mathrm{CD}$ and $\mathrm{CB}[8]$. In this threecomponent system, molecule 1 can form two stable supramolecular complexes, $1 \subset \beta$-CD in its trans form and $\mathbf{1} \subset \mathrm{CB}[8]$ in its cis form. Upon photo-switching, $\mathbf{1}$ could be reversibly transferred between the two host cavities. By synthetic modification of the photo-switch ${ }^{[31]}$, the now still rather incomplete cis-to-trans conversion may be further optimized. This competitive inclusion can be further used in the development of photo-responsive host-guest supramolecular systems, such as cargo carriers, molecular logic gates, and other supramolecular materials.

\subsection{Acknowledgements}

Prof. Jurriaan Huskens is gratefully acknowledged for discussions on host-guest complexation. Dr. Alberto Juan Ruiz Del Valle is gratefully acknowledged for performing the in-situ photo-switching NMR experiments.

\subsection{Supporting information}

\subsubsection{Materials and methods}

$\mathrm{CB}[8]$ was purchased from Strem Chemicals and all other chemicals were purchased from 
Sigma-Aldrich. They were used as purchased, without further purification.

A Hönle bluepoint LED lamp was employed as a UV light source $(\lambda=365 \mathrm{~nm})$ for $e x$ situ NMR experiments and UV/Vis absorption measurements. Blue light $(\lambda=420 \mathrm{~nm})$ was provided by an Edmund MI-150 visible lamp equipped with an Edmund $420 \pm 2 \mathrm{~nm}$ filter. The photo-stationary states were determined by ${ }^{1} \mathrm{H}$ NMR in $\mathrm{D}_{2} \mathrm{O}(\mathrm{c}=0.5 \mathrm{mM})$.

For in-situ NMR experiments, a T7070 1 PowerStar LED lamp from ILS was employed as a UV light source, a N5050 1 PowerStar LED lamp from ILS was employed as a blue light source, and the optical fibers were purchased from Edmund.

Ex-situ NMR data was recorded on a $400 \mathrm{MHz}$ Bruker spectrometer. In-situ NMR data was measured on a $600 \mathrm{MHz}$ Bruker spectrometer by water suppression mode. The light was introduced via an optical fiber into the NMR tube. The total irradiation time was $30 \mathrm{~min}$, and spectra were recorded every $4 \mathrm{~min}$. All chemical shifts were referenced to the internal $\mathrm{CDCl}_{3}$ signal at $7.24 \mathrm{ppm}, \mathrm{CD}_{3} \mathrm{CN}$ at $1.94 \mathrm{ppm}, \mathrm{D}_{2} \mathrm{O}$ at $4.80 \mathrm{ppm}$ or TSP at $0 \mathrm{ppm}$ (only for water suppression). For UV-Vis absorption and ITC titrations, stock solutions were prepared in MilliQ water. UV/Vis spectra were recorded in a conventional quartz cell in a Perkin Elmer Lambda 850 spectrophotometer.

ITC titrations were performed using a MicroCal ${ }^{\mathrm{TM}}$ VPC microcalorimeter. Each ITC titration was performed with 60 injections of $5 \mu$ l each. A solution of $\beta$-CD in water $(c=2.5$ $\mathrm{mM})$ was titrated to the trans $\mathbf{- 1}$ or $\operatorname{cis} \mathbf{- 1}$ solution $(\mathrm{c}=0.25 \mathrm{mM})$. Alternatively, a solution of trans $\mathbf{- 1}$ or $c i s-1(\mathrm{c}=0.5 \mathrm{mM})$ was titrated to a $\mathrm{CB}[8]$ solution $(\mathrm{c}=0.05 \mathrm{mM})$. 


\subsubsection{Synthesis}

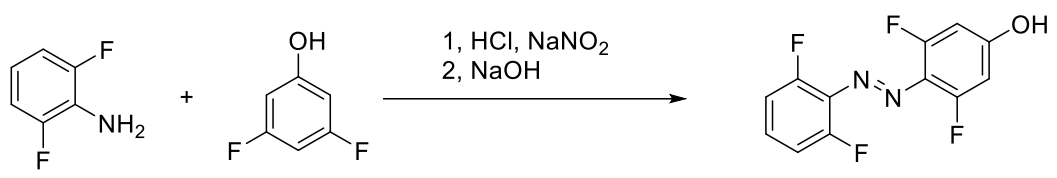

Schema S4.1. Synthesis of ortho-fluorinated benzene-azophenol.

Ortho-fluorinated benzene-azophenol (FA-OH) was synthesized via diazonium reaction. 2,6-difluoro aniline ( $0.387 \mathrm{~g}, 3 \mathrm{mmol}, 1$ eq.) and $1.05 \mathrm{ml}$ of concentrated hydrochloric acid were dissolved in $7.5 \mathrm{ml}$ of water. This solution was cooled down to $0{ }^{\circ} \mathrm{C}$ and stirred. To this stirred solution was added a solution of sodium nitrite $(0.249 \mathrm{~g}, 3.3 \mathrm{mmol}, 1.1 \mathrm{eq}$.$) in water$ (4.5 ml) dropwise. The temperature was controlled between $0{ }^{\circ} \mathrm{C}$ to $5{ }^{\circ} \mathrm{C}$ during the addition. Resulting suspension was added to an ice bath cooled solution of 3,5-diluoro phenol (0.429 g, $3.3 \mathrm{mmol}, 1.1$ eq.) and sodium hydroxide $(0.400 \mathrm{~g}, 10 \mathrm{mmol}, 3.3 \mathrm{eq}$.$) in of water (6 \mathrm{ml})$ dropwise. The suspension was acidified with $1 \mathrm{M} \mathrm{HCl}$ to $\mathrm{pH}=2$, extracted with DCM. Organic layer was collected, dried over $\mathrm{MgSO}_{4}$. Then the DCM was evaporated, the mixture was objected to a Si-chromatographic column, $0.432 \mathrm{~g}$ product was isolated as dark red solid, yield $=53 \% .{ }^{1} \mathrm{H}$ NMR in $\mathrm{CD}_{3} \mathrm{CN}: \delta=6.69(\mathrm{dd}, J=11.2,2.8 \mathrm{~Hz}, 2 \mathrm{H}), 7.19(\mathrm{t} . J=8.8 \mathrm{~Hz}, 2 \mathrm{H})$, $7.49 \mathrm{ppm}(1 \mathrm{H}, \mathrm{m})$.
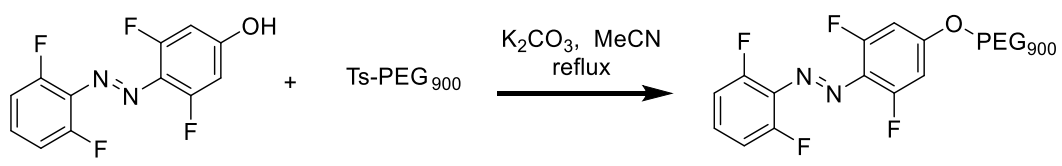

Schema S4.2. Synthesis of compound 1.

In the second step, a mixture of FA-OH $(0.084 \mathrm{~g}, 0.31 \mathrm{mmol}, 1.5 \mathrm{eq}),. \mathrm{K}_{2} \mathrm{CO}_{3}(0.058 \mathrm{~g}$, $0.42 \mathrm{mmol}, 2$ eq. $)$ and Ts-PEG ( $\mathrm{mw}=900,0.058 \mathrm{~g}, 0.42 \mathrm{mmol}, 1$ eq.) in $7 \mathrm{ml}$ of $\mathrm{MeCN}$ was 
stirred and refluxed for overnight. Then the reaction mixture was cooled down to room temperature, the $\mathrm{MeCN}$ was removed under vacuum. The residues were dissolved in DCM, washed with water. The organic phase was collected and dried over $\mathrm{MgSO}_{4}$. Then the $\mathrm{DCM}$ was evaporated, the mixture was objected to a Si-chromatographic column, 0.066g product was isolated as dark red liquid, yield $=30 \%$.

${ }^{1} \mathrm{H}$ NMR in $\mathrm{CDCl}_{3}: \delta=3.33(\mathrm{~s}, 3 \mathrm{H}), 3.50(\mathrm{~m}, 2 \mathrm{H}), 3.59(\mathrm{~m}, 48 \mathrm{H}), 3.83(\mathrm{t}, J=4.8 \mathrm{~Hz}, 2 \mathrm{H})$, 4.14(t, $J=4.4 \mathrm{~Hz}, 2 \mathrm{H}), 6.68(\mathrm{~d}, J=11.2 \mathrm{~Hz}, 2 \mathrm{H}), 6.99(\mathrm{t}, J=8.8 \mathrm{~Hz}, 2 \mathrm{H}), 7.27 \mathrm{ppm}(\mathrm{m}, 1 \mathrm{H})$.

${ }^{13} \mathrm{C}$ NMR in $\mathrm{CDCl}_{3}: \delta=161.88,157.40(\mathrm{C}-\mathrm{F}, \mathrm{d}, J=190 \mathrm{~Hz}), 155.11(\mathrm{C}-\mathrm{F}, \mathrm{d}, J=185 \mathrm{~Hz})$, $132.08,130.41,126.07,112.51,99.52,71.93,70.95,70.56,68.91,59.04$ ppm.

From the ${ }^{1} \mathrm{H}-\mathrm{NMR}$ the molecular weight was calculated as 870.35 , which confirmed that there is in average 13.5 ethylene glycol (EG) unit on the PEG moiety. Mass spectrum were founded signals at $\mathrm{m} / \mathrm{z}=724.89,768.88,812.88,856.87,900.87$, corresponding to 1 with 10 to 14 EG units successively.

The photo stationary state (PSS) was determined by ${ }^{1} \mathrm{H}$ NMR in $\mathrm{D}_{2} \mathrm{O}(\mathrm{c}=0.5 \mathrm{mM})$. In the initial state, the ratio between trans- and cis- isomers was trans- : cis- $=94: 6$. Under irradiation by UV light $(365 \mathrm{~nm})$ for 15 minutes, the UV PSS was achieved with a ratio as trans- : cis- $=10: 90$. Then the sample was irradiated by $420 \mathrm{~nm}$ blue light for cis- to transconversion. The $420 \mathrm{~nm}$ PSS was achieved after 20 minutes-irradiation with a ratio as trans- : $c i s-=57: 43$. 


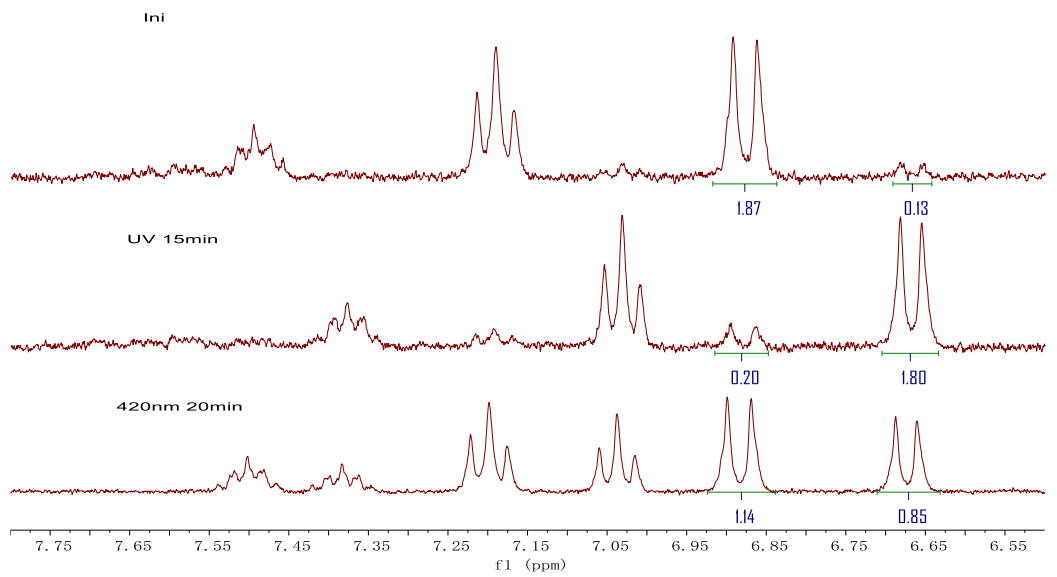

Figure S4.1. PSS determination of 1 by ${ }^{1} \mathrm{H}$ NMR in $\mathrm{D}_{2} \mathrm{O}(\mathrm{c}=0.5 \mathrm{mM})$.

\subsubsection{Complexation of 1 in $\beta-C D$}

a)

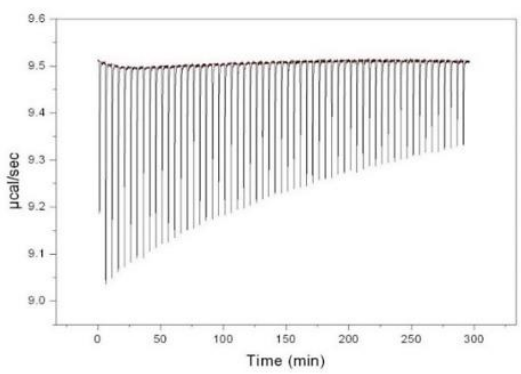

b)

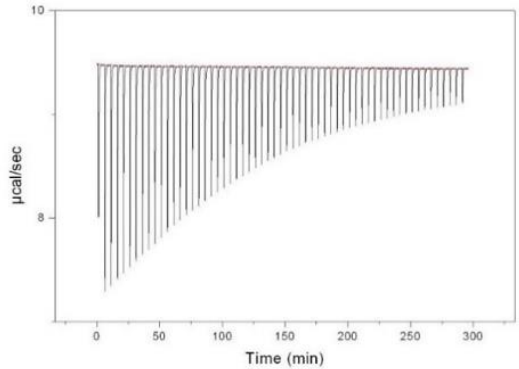

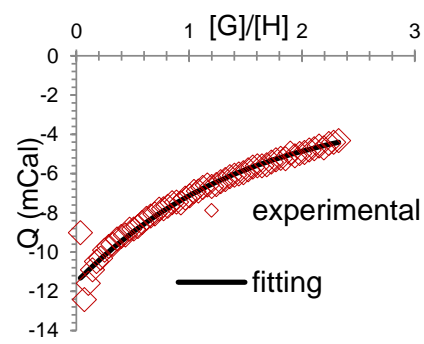

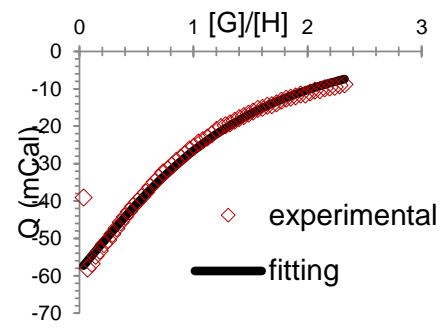

Figure S4.2. ITC titration of $1 \subset \beta-C D$ complex. a) $\beta-C D$ to trans-1, b) $\beta-C D$ to $c i s-1$ 


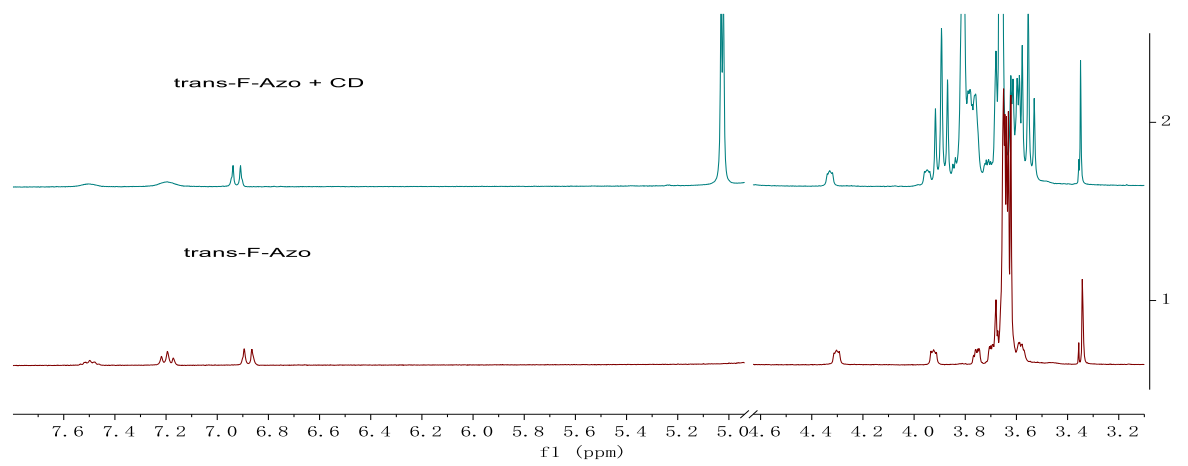

Figure $\mathbf{S 4 . 3}{ }^{1} \mathrm{H}$ NMR of trans -1 and trans -1 with $\beta-\mathrm{CD}$ mixture in $\mathrm{D}_{2} \mathrm{O}(\mathrm{c}=0.5 \mathrm{mM})$.

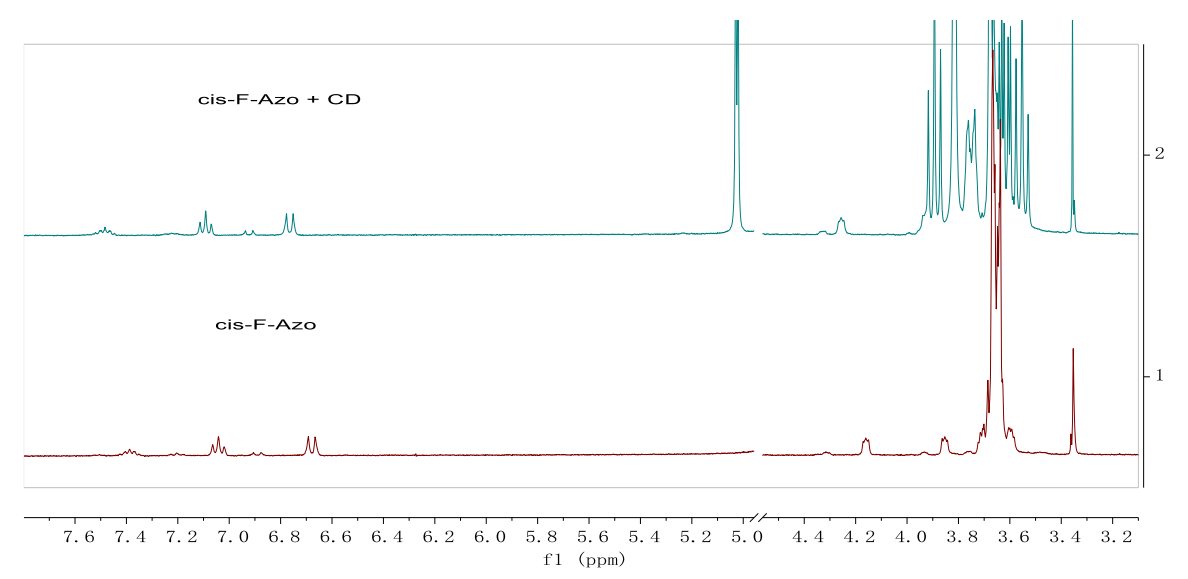

Figure S4.4. ${ }^{1} \mathrm{H}$ NMR of $c i s-1$ and $c i s-1$ with $\beta-\mathrm{CD}$ mixture in $\mathrm{D}_{2} \mathrm{O}(\mathrm{c}=0.5 \mathrm{mM})$.

The stoichiometry was studied by ${ }^{1} \mathrm{H}$ NMR Job plot titration, the total concentration of $\mathbf{1}$ and $\beta-\mathrm{CD}$ was set as $0.5 \mathrm{mM}$. For both the trans- and cis-1, the maximum of $\Delta \delta^{*}[\mathrm{CD}]$ was all appeared at $\mathrm{r}=0.5$, which confirmed that both the trans- and cis-1 could form a 1:1 complexation with $\beta-\mathrm{CD}($ trans $-\mathbf{1} \subset \beta-\mathrm{CD}$, cis-1 $\subset \beta-\mathrm{CD})$. 


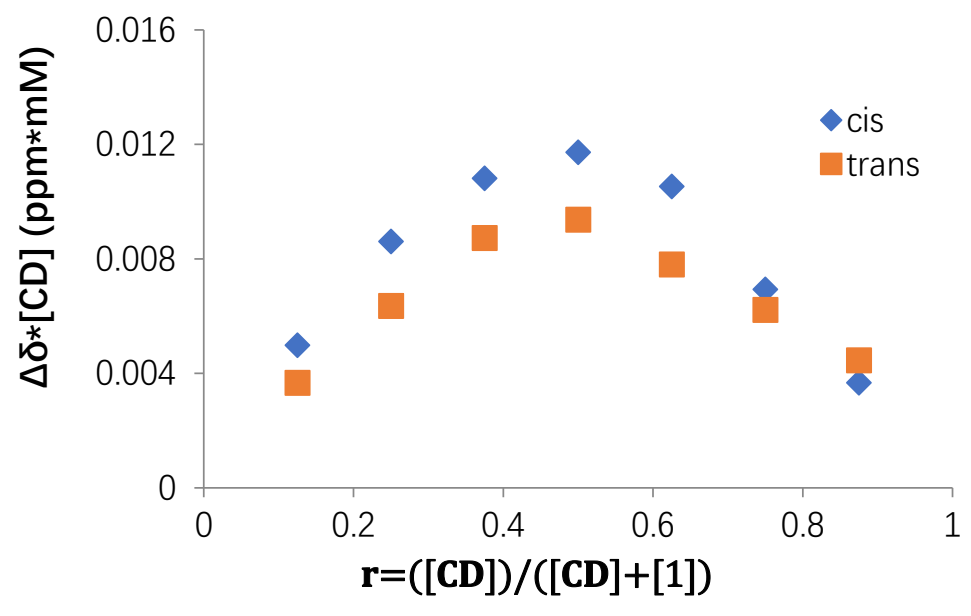

Figure S4.5. Job plot for stoichiometry of $1 \subset \beta-C D$ complex.

To determine the binding affinity of complex trans-1 $\subset \beta$-CD and cis-1 $\subset \beta-\mathrm{CD},{ }^{1} \mathrm{H}$ NMR titration experiments were performed. The concentration of the guest molecule was kept as constant $(\mathrm{c}=0.5 \mathrm{mM})$, a series of samples with different ratio of host molecule were measured. The binding constant $\mathrm{k}$ was calculated by Benesi-Hildebrand's method:

$$
\frac{1}{\Delta \delta o b s}=\frac{1}{K \Delta \delta[\mathrm{H}]}+\frac{1}{\Delta \delta}
$$

Where the $[\mathrm{H}]$ is the concentration of host molecule $\beta-\mathrm{CD}, \Delta \delta$ is the shift of the signal from the proton on 1 . The results suggested that the cis-1 has a higher binding affinity to $\beta$ $\mathrm{CD}\left(\mathrm{k}=4.7^{*} 10^{3} \mathrm{M}^{-1}\right)$ than the trans $-1\left(\mathrm{k}=1.5^{*} 10^{3} \mathrm{M}^{-1}\right)$. 


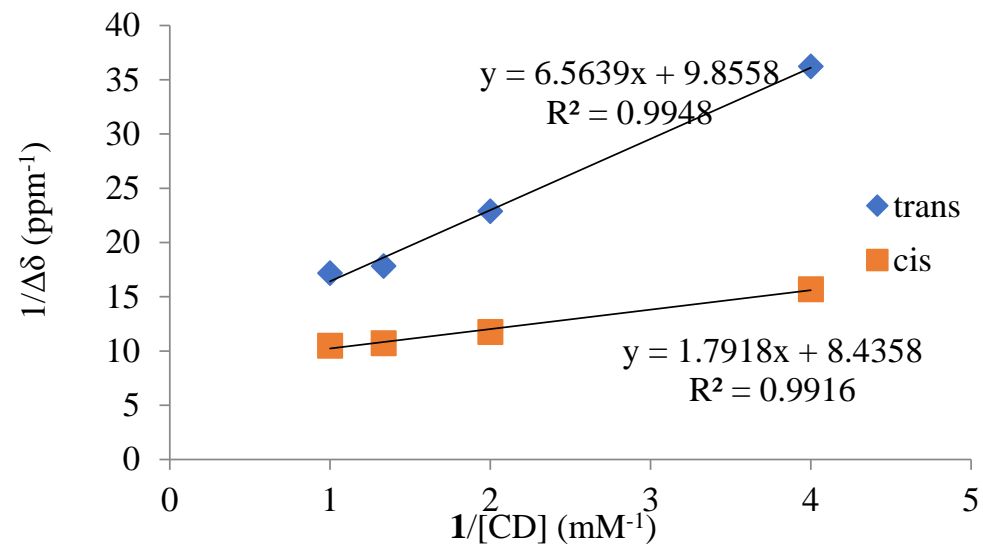

Figure S4.6. Binding constant calculation by Benesi-Hildebrand's method of $1 \subset \beta$-CD complex.

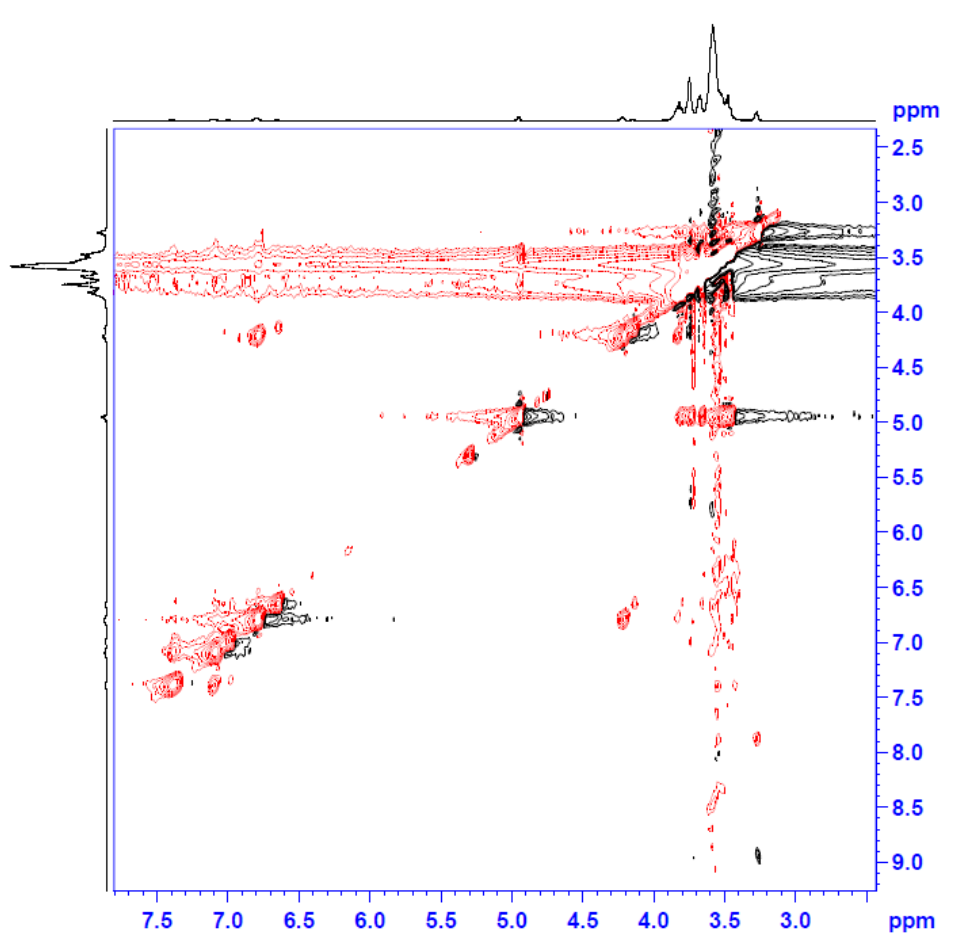

Figure S4.7. NOESY-NMR spectrum of trans -1 and $\beta-\mathrm{CD}$ in $\mathrm{D}_{2} \mathrm{O}$. (trans $\left.-1: \beta-\mathrm{CD}=1: 1, \mathrm{c}=1 \mathrm{mM}\right)$. 


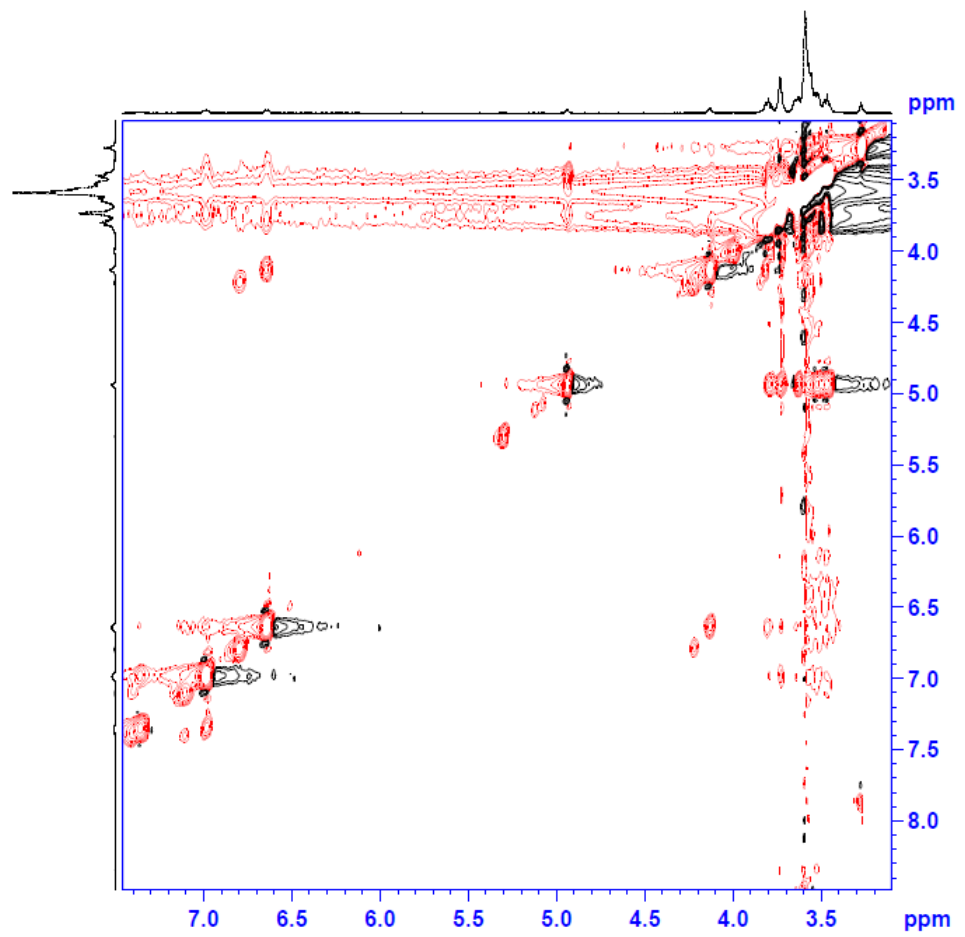

Figure S4.8. NOESY-NMR spectrum of $c i s-\mathbf{1}$ and $\beta-\mathrm{CD}$ in $\mathrm{D}_{2} \mathrm{O}$. $($ cis-1 $: \beta-\mathrm{CD}=1: 1, \mathrm{c}=1 \mathrm{mM})$.

\subsubsection{Complexation of 1 in $\mathrm{CB}[8]$}

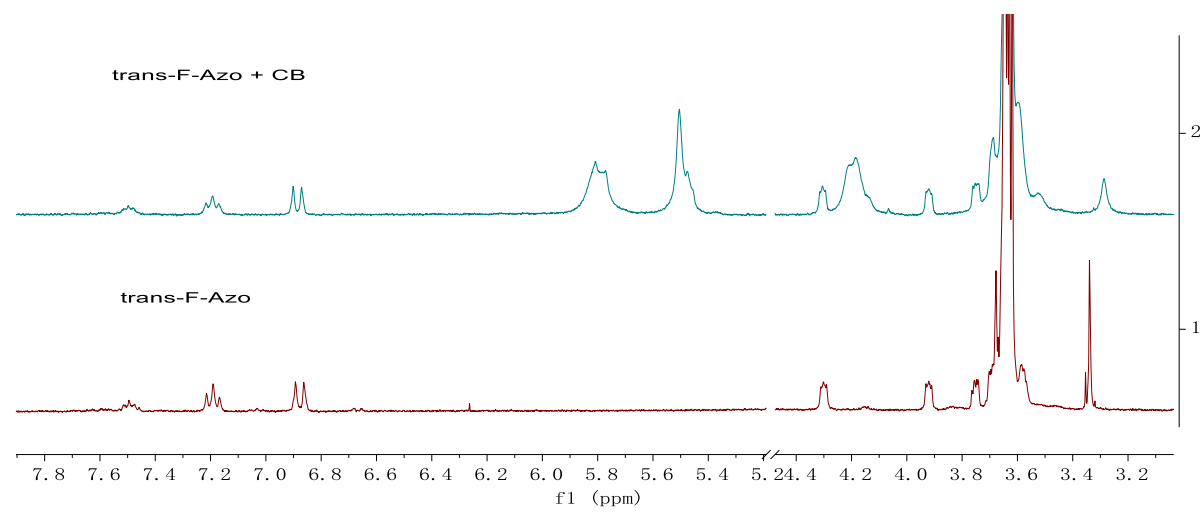

Figure S4.9. ${ }^{1} \mathrm{H}$ NMR of trans-1 and trans -1 with $\mathrm{CB}[8]$ mixture in $\mathrm{D}_{2} \mathrm{O}(\mathrm{c}=0.5 \mathrm{mM})$. 


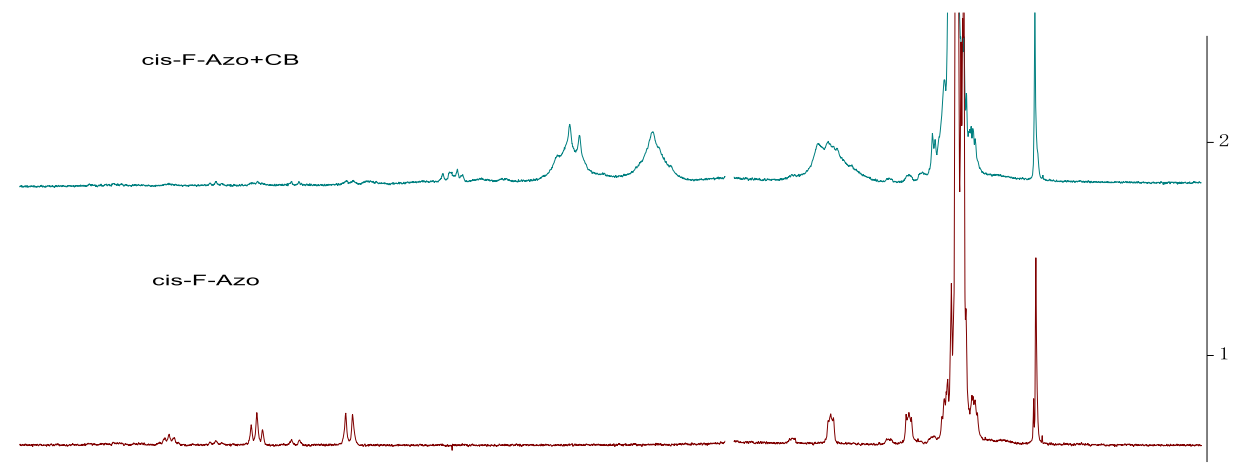

$\begin{array}{llllllllllllllllllllllllllllll}7.8 & 7.6 & 7.4 & 7.2 & 7.0 & 6.8 & 6.6 & 6.4 & 6.2 & 6.0 & 5.8 & 5.6 & 5.4 & 5.2 & 4.4 & 4.2 & 4.0 & 3.8 & 3.6 & 3.4 & 3.2 & 3.0 & 2.8\end{array}$ f1 (ppm)

Figure S4.10. ${ }^{1} \mathrm{H}$ NMR of $c i s-1$ and $c i s-1$ with $\mathrm{CB}[8]$ mixture in $\mathrm{D}_{2} \mathrm{O}(\mathrm{c}=0.5 \mathrm{mM})$.

a

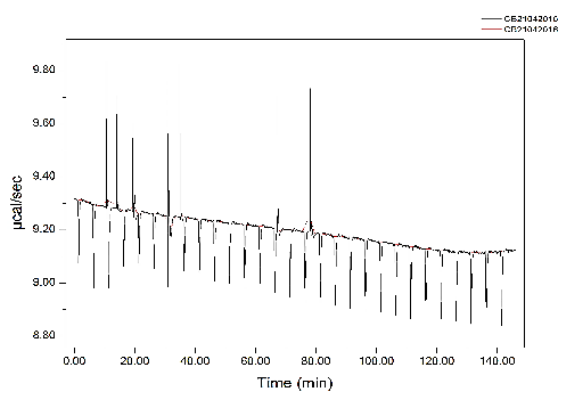

$\mathrm{b}$
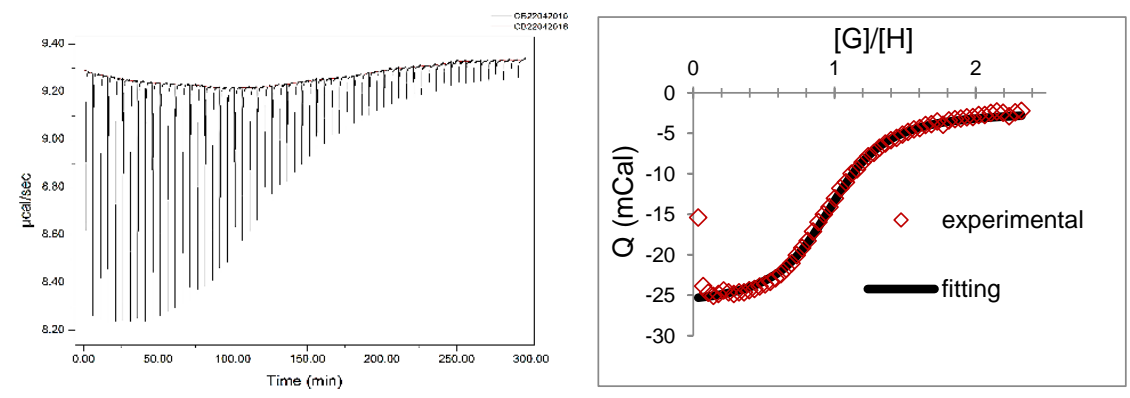

Figure S4.11. ITC titration of $1 \subset \mathrm{CB}[8]$ complex. a) trans-1 to $\mathrm{CB}[8]$, b) cis-1 to $\mathrm{CB}[8]$ 


\subsubsection{In-situ switching of 1 in the absence of $\mathrm{CB}[8]$}

a)

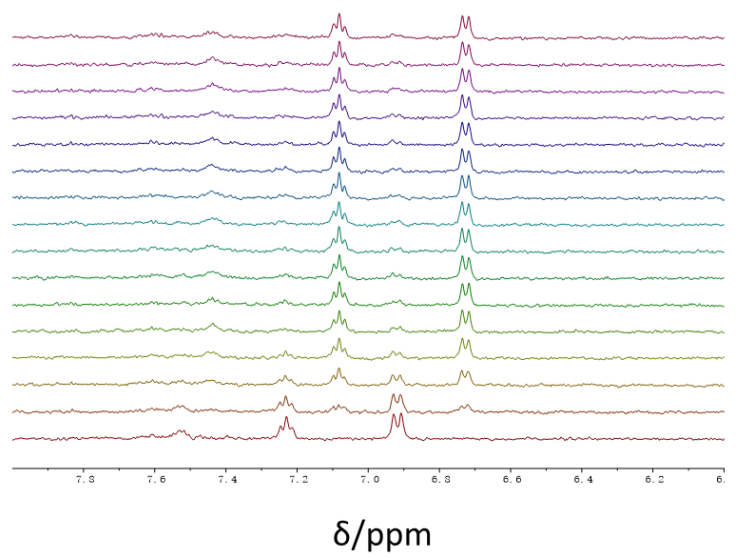

b)

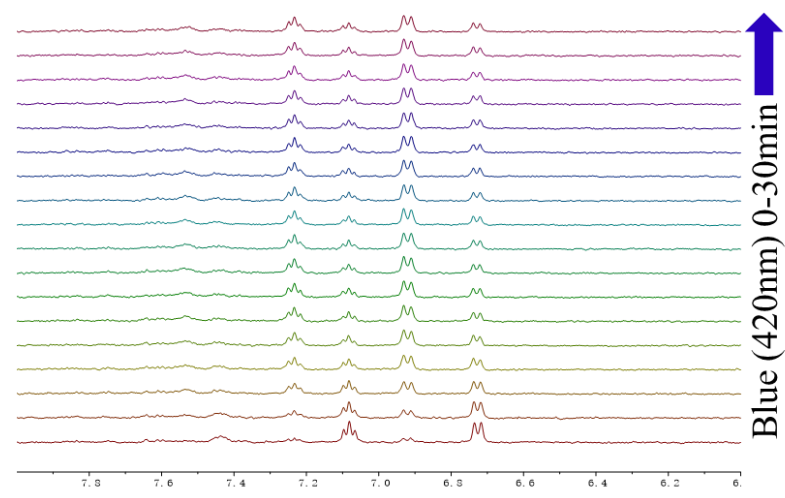

$\delta / p p m$

Figure S4.12. ${ }^{1} \mathrm{H}$ NMR spectra of the in-situ switching experiments of $\mathbf{1}: \beta-\mathrm{CD}=1: 1$ in $\mathrm{D}_{2} \mathrm{O}(\mathrm{c}=0.05$ $\mathrm{mM}$ ), showing (from bottom to top for 0-30 min of illumination) the a) trans- to cis-and b) cis- to trans- conversions as induced by illumination with $\lambda=365 \mathrm{~nm}$ and $\lambda=420 \mathrm{~nm}$ light, respectively. 


\subsection{References}

[1] D. H. Qu, Q. C. Wang, Q. W. Zhang, X. Ma, H. Tian, Chem. Rev., 2015, 115, 7543.

[2] L. Graña-Suárez, W. Verboom, J. Huskens, Chem. Commun., 2016, 52, 2597.

[3] A. Harada, R. Kobayashi, Y. Takashima, A. Hashidzume, H. Yamaguchi, Nature Chem., 2011, 3, 34.

[4] K. Iwaso, Y. Takashima, A. Harada, Nature Chem., 2016, 8, 625.

[5] R. Mejia-Ariza, G. A. Kronig, J. Huskens, Beilstein J. Org. Chem., 2015, 11, 2388.

[6] C. Stoffelen, J. Voskuhl, P. Jonkheijm, J. Huskens, Angew. Chem. Int. Ed., 2014, 53, 3400.

[7] M. M. Russew, S. Hecht, Adv. Mater., 2010, 22, 3348.

[8] A. Mulder, A. Jukovic, L. N. Lucas, J. van Esch, B. L. Feringa, J. Huskens, D. N. Reinhoudt, Chem. Comm., 2002, 22, 2734.

[9] R. Klajn, Chem. Soc. Rev., 2014, 43, 148.

[10] X. Su, I. Aprahamian, Chem. Soc. Rev., 2014, 43, 1963.

[11] B. L. Feringa, W. R. Browne, Molecular switches. Second Edition. Wiley-VCH: Weinheim; 2011.

[12] H. M. D. Bandara, S. C. Burdette, Chem. Soc. Rev., 2012, 41, 1809.

[13] S. Dong, B. Zheng, F. Wang, F. Huang, Acc. Chem. Res., 2014, 47, 1982.

[14] M. R. de Jong, J. Huskens, D. N. Reinhoudt, Chem. Eur. J., 2001, 7, 4164.

[15] B. J. Ravoo, J-C. Jacquier, Macromolecules, 2002, 35, 6412.

[16] A. Harada, Y. Takashima, M. Nakahata, Acc. Chem. Res., 2014, 47, 2128.

[17] A. Ueno, K. Takahashi, T. Osa, J. Chem. Soc. Chem. Comm., 1980, 17, 837.

[18] Y. Liu, C. Yu, H. Jin, B. Jiang, X. Zhu, Y. Zhou, Z. Lu, D. Yan, J. Am. Chem. Soc., 2013, 135,4765 .

[19] P. Wan, Y. Wang, Y. Jiang, H. Xu, X. Zhang, Adv. Mater., 2009, 21, 4362.

[20] O. Roling, L. Stricker, J. Voskuhl, S. Lamping, B. J. Ravoo, Chem. Commun., 2016, 52, 1964.

[21] G. Yu, K. Jie, F. Huang, Chem. Rev., 2015, 115, 7240.

[22] J. Lagona, P. Mukhopadhyay, S. Chakrabarti, L. Isaacs, Angew. Chem. Int. Ed., 2005, $44,4844$.

[23] L. Isaacs, Acc. Chem. Res., 2014, 47, 2052.

[24] J. Liu, Y. Lan, Z. Yu, C. S. Y. Tan, R. M. Parker, C. Abell, O. A. Scherman, Acc. Chem. Res., 2017, 50, 208.

[25] L. P. Cao, M. Šekutor, P. Y. Zavalij, K. Mlinaric-Majerski, R. Glaser, L. Isaacs, Angew. 
Chem. Int. Ed., 2014, 53, 988.

[26] F. Tian, D. Jiao, F. Biedermann, O. A. Scherman, Nature Comm. 2012, 3, 1207.

[27] H-B. Cheng, Y-M. Zhang, C. Xu, Y. Liu, Sci. Rep., 2014, 4, 4210.

[28] J. del Barrio, P. N. Horton, D. Lairez, G. O. Lloyd, C. Toprakcioglu, O. A. Scherman, J. Am. Chem. Soc., 2013, 135, 11760.

[29] E. Mattia, S. Otto, Nature Nanotech., 2015, 10, 111.

[30] D. Bléger, J. Schwarz, A. M. Brouwer, S. Hecht, J. Am. Chem. Soc., 2012, 134, 20597.

[31] C. Knie, M. Utecht, F. Zhao, H. Kulla, S. Kovalenko, A. M. Brouwer, P. Saalfrank, S. Hecht, D. Bléger, Chem. Eur. J., 2014, 20, 16492.

[32] L. Zhang, H. Zhang, F. Gao, H. Peng, Y. Ruan, Y. Xu, W. Weng, RSC Adv., 2015, 5, 12007.

[33] K. Bouchemal, S. Mazzaferro, Drug Discov. Today, 2012, 17, 623. 


\section{Chapter 5}

\section{Host-guest complexation mediated by ortho- fluorinated azobenzenes in supramolecular nanoparticles}

Multivalency effect has recently allowed building up supramolecular architectures by enhancing weak host-guest interactions. Meanwhile, azobenzenes are molecular photoswitches that can be employed as a dynamic building blocks in host-guest chemistry due to their photo controllable binding and release with macrocyclic host cavities. In last chapter, we investigated the competitive binding of an ortho-fluorinated azobenzene with two different host cavities, $\beta$-cyclodextrin ( $\beta$-CD) and cucurbit[8] uril (CB[8]). In this chapter, we involved this photo-switch in multivalent host-guest self-assemblies as a multivalent guest, with $\beta$-CD being a multivalent host and $C B[8]$ being a monovalent host. The final aim is to build dynamic supramolecular nanoparticles that display photo-triggered bi-stable assembly and dissociation. 


\subsection{Introduction}

Supramolecular chemistry allows the generation of highly ordered functional architectures by non-covalent interactions with two basic concepts being involved: selfassembly and specific binding based on molecular recognition, i.e. host-guest complexation $^{[1-6]}$. In particular, supramolecular chemistry offers new means to synthetic nanoparticles with 1) controllable sizes and morphologies, 2) bio-compatibility with low toxicity, 3) convenient surface charges and chemistry for enhanced physiological stability and 4) long circulation time $\mathrm{e}^{[8]}$, enabling various of functions in bio-medical applications ${ }^{[9-10]}$.

$\beta$-cyclodextrin ( $\beta$-CD) is one of the most widely used host building block in biomedical applications, as it can recognize and bind hydrophobic guest molecules ${ }^{[11-18]}$. However, its binding affinity is always relatively low $\left(10^{3} \sim 10^{4} \mathrm{M}^{-1}\right)^{[18]}$. In nature, weak interactions are enhanced by multivalency ${ }^{[19-21]}$. When both the host and guest have multiple binding sites, after formation of the first host-guest complex, the remaining binding sites are limited intramolecularly in a highly concentrated volume, and the whole system presented a strongly enhanced overall binding affinity ${ }^{[22-23]}$.

Tseng and co-workers have developed supramolecular nanoparticles by multivalent hostguest interaction ${ }^{[8]}$ : polyamidoamine (PAMAM) dendrimer was terminally functionalized with guest building block adamantyl as multivalent guest, branched polymer polyethylenimine (PEI) was grafted with $\beta$-CD as multivalent host (CD-PEI). In the formation of supramolecular nanoparticles, CD-PEI works as a cross-linker to join the dendrimers together by adamantyl $\subset \beta-\mathrm{CD}$ complexation, monovalent guest molecules were employed to fulfill all the host binding sites to terminate the multivalent host-guest binding. 
By tuning the ratio between the three components, the size of supramolecular nanoparticles can be easily controlled. The reserved binding sites allowed the nanoparticles carrying cargos for various functions ${ }^{[24-28]}$.

Stimulus responsive host-guest complexes were applied to this architecture to form supramolecular nanoparticles with controllable assembly-disassembly and to achieve stimuli triggered cargo release ${ }^{[29-30]}$. We focus on light as spatial and temporary controllable stimulus to build up dynamic material with molecular photo-switches ${ }^{[31-33]}$. Azobenzene undergoes UV triggered trans-to-cis isomerization, causes changes on both conformation and dipole moment, the reverse conversion can be triggered by visible light or thermal condition ${ }^{[34]}$. Trans- azobenzene could form host-guest complex with macrocyclic host molecule $\beta-\mathrm{CD}^{[35-}$ ${ }^{39]}$, or form hetero ternary complex with methyl viologen in cucurbit[8]uril $(\mathrm{CB}[8])^{[40-41]}$, both the complexes will be dissociated by trans-to-cis isomerization. However, for some applications, the lack of thermal stability of the $c i s$-isomer remains a limitation. For example, in a recent report, methyl viologen azobenzene $\subset \mathrm{CB}[8]$ complex was incorporated into multivalent supramolecular nanoparticles, photo-isomerization of azobenzene results in disassembly of the host-guest complex so well as the nanoparticle ${ }^{[42]}$. Then in 10 hours, the disassembled building blocks were self-assembled and resulting in supramolecular nanoparticles again by the cis-to-trans thermal relaxation. To keep the disassembled state, a continuously UV stimulus input is required ${ }^{[43]}$.

Recently, ortho-fluorinated azobenzenes (FA) were shown to display thermal stability in their $c i s-$ form ${ }^{[44-45]}$. As seen in Chapter 4, FA can form host-guest complex with $\beta$-CD in both their trans- and cis- state, with the cis- isomer having a higher binding affinity ( $K=4.8$ 
$\left.\times 10^{3} \mathrm{M}^{-1}\right)$ than the trans- form $\left(K=2.3 \times 10^{3} \mathrm{M}^{-1}\right)$. With another host cavity $\mathrm{CB}[8]$, the transFA could not form ternary complex with methyl viologen, however a stable complex cisFA $\subset \mathrm{CB}[8]$ with binding affinity $K=4.9 \times 10^{5} \mathrm{M}^{-1}$ was observed. When both the $\beta$-CD and

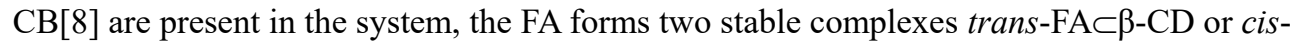

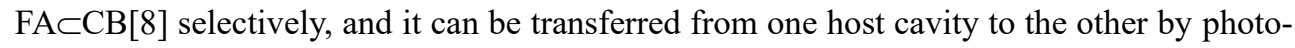
isomerization $^{[46-47]}$. Here, we involved a fluorinated azobenzene in multivalent architectures, with the aim to design bi-stable supramolecular systems as well as to study competitive hostguest interaction in multivalent systems. The fluorinated azobenzene was immobilized on a PAMAM dendrimer as multivalent guest, and the branched polymer CD-PEI was employed as multivalent host, $\mathrm{CB}[8]$ was employed as monovalent host [Figure 5.1a]. Two systems were investigated:

a) a bi-component system with FA-PAMAM and CD-PEI. As the cis-FA has higher binding affinity to $\beta$-CD than its trans-form, we expect that neither the host guest complex nor the supramolecular particle will be formed in the trans-state, but the cis- state they will [Figure 5.1b];

b) a tri-component system with FA-PAMAM, CD-PEI and CB[8]. Considering that the binding affinity of $c i s-\mathrm{FA} \subset \mathrm{CB}[8]$ is about two orders of magnitudes higher than $c i s-\mathrm{FA} \subset \beta$ $\mathrm{CD}$, and that there is no interaction between $\mathrm{CB}[8]$ and trans-FA, the formation of supramolecular nanoparticles was expected as a result of trans-FA $\subset \beta-\mathrm{CD}$ complexation, and its dissociation was expected by $c i s-\mathrm{FA} \subset \mathrm{CB}[8]$ complexation [Figure 5.1c]. 
a)

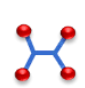

$\mathrm{FA}_{4} \mathrm{G}_{0}$

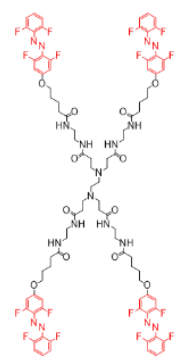

b)

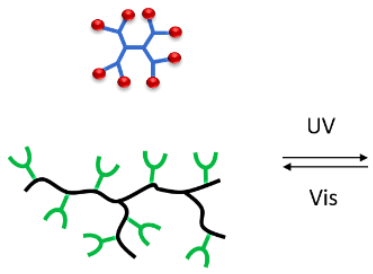

trans- state

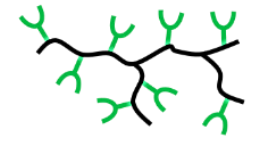

CD-PEI

$\mathrm{CB}[8]$
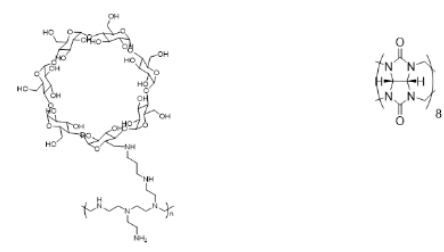

c)

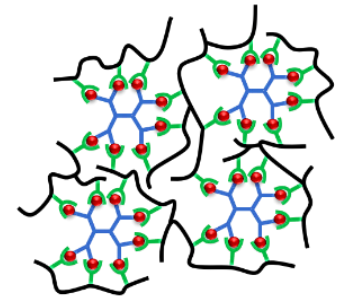

cis- state

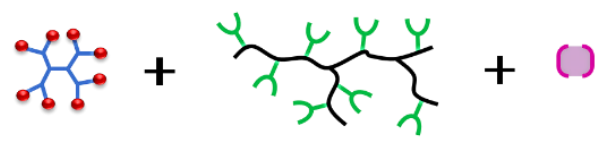

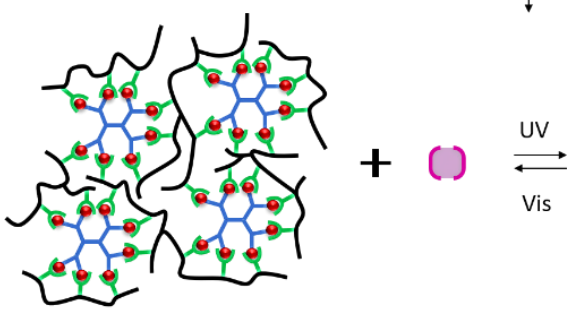

trans- state

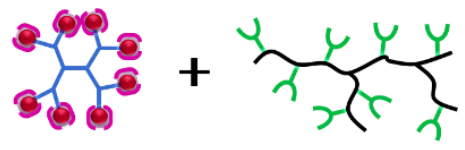

cis- state

Figure 5.1. a) Chemical compounds employed in this chapter. b) FA-PAMAM with CD-PEI bicomponent system. c) FA dendrimer, CD-PEI and $\mathrm{CB}[8]$ tri-components competitive binding system. 


\subsection{Results and discussion}

Two multivalent guest dendrimers $\mathrm{FA}_{8}-\mathrm{PAMAM}-\mathrm{G}_{1}\left(\mathrm{FA}_{8} \mathrm{G}_{1}\right)$ with eight fluorinated azobenzene units and $\mathrm{FA}_{4}-\mathrm{PAMAM}-\mathrm{G}_{0}\left(\mathrm{FA}_{4} \mathrm{G}_{0}\right)$ with four fluorinated azobenzene units were synthesized [Figure S5.1] $\beta$-CD was immobilized on PEI polymers $\left(\mathrm{M}_{\mathrm{w}}=10 \mathrm{k}\right.$ Da), resulting in multivalent host with $8 \beta-C D$ units per PEI in average [Figure S5.2]. The $\mathrm{FA}_{8} \mathrm{G}_{1}$ and $\mathrm{FA}_{4} \mathrm{G}_{0}$ were prepared as highly concentrated DMSO stock solution, CD-PEI and CB[8] were prepared as milli-Q water stock solution respectively. In a normal sample, the DMSO ratio was set as lower than $0.5 \%$ per volume.

\subsubsection{A bi-component system}

Firstly, we investigated bi-component system with FA-PAMAM with CD-PEI. Considering that the F-Azo has higher binding affinity to CD in the cis- form than the transform, in the di-component system we expect that the FA would not form host-guest complex in its trans-form, and self-assembled nanoparticles was generated in its cis-form.

In all the samples, the ratio of host and guest moieties was set as $[\mathrm{CD}]:[\mathrm{FA}]=1: 1$. Since both the FA-PAMAM and the CD-PEI were positively charged, the formation of supramolecular nanoparticles will be hindered by the electrostatic repulsive effect ${ }^{[29]}$. Thus, the environmental ionic strength is an important factor for the building blocks to overcome the repulsive interaction. We started with $\mathrm{FA}_{8} \mathrm{G}_{1}$ with concentration as $0.1 \mathrm{mM}$ per FA unit, ionic strength was tuned by $\mathrm{NaCl}$ solution from $[\mathrm{NaCl}]=0 \mathrm{M}$ to $0.3 \mathrm{M}$. To prepare a general sample, the CD-PEI stock solution was diluted by milliQ water and $\mathrm{NaCl}$ solution, led to a CD-PEI solution in proper concentration and ionic strength, then the $F_{8} G_{1} D M S O$ solution was added, the resulting mixture was sonicated for 15 minutes. At each condition, there are 
two samples prepared in parallel, one was measured as the initial state with trans-FA isomers, the other was illuminated by UV light $(\lambda=365 \mathrm{~nm})$ for one minute after the sonication [Figure S5.3]. The samples were analyzed by dynamic light scattering (DLS) at 20 min, 1 hour, 2 hours and 3 hours after mixing and sonication.

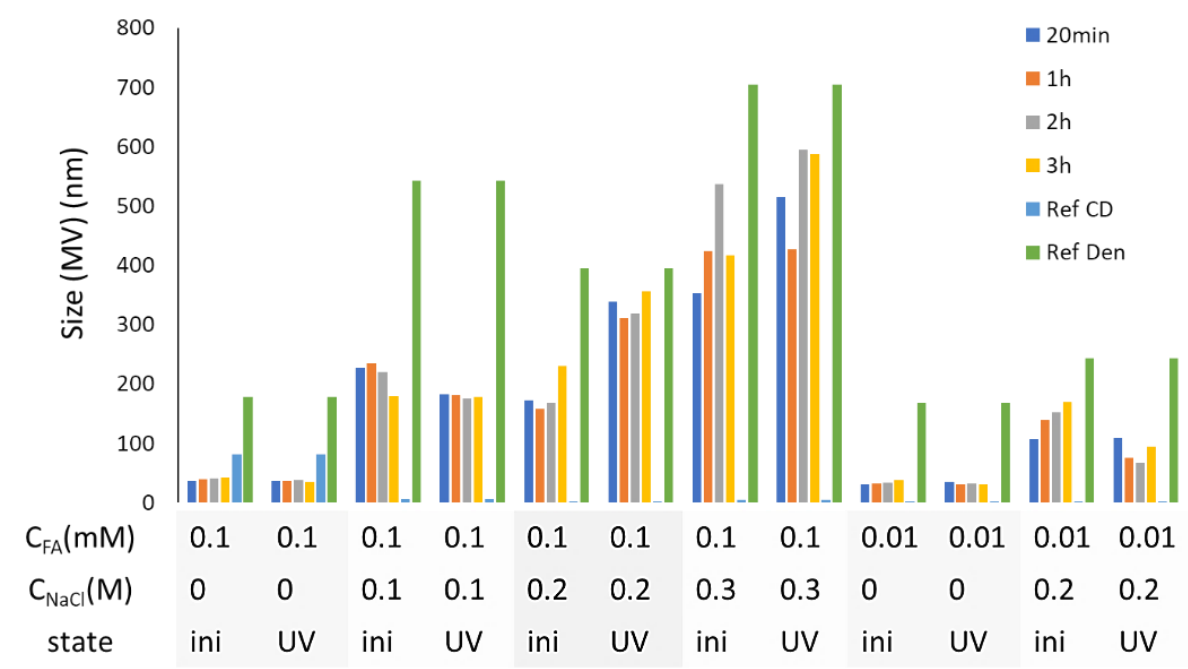

Figure 5.2. DLS analysis for $\mathrm{FA}_{8} \mathrm{G}_{1} \mathrm{CD}-\mathrm{PEI}$ bi-component system.

Nano-objects were detected in for all the different ionic strengths that were investigated [Figure 5.2]. All the signals from the sample mixtures did not show same size as the reference sample $\left(\mathrm{FA}_{8} \mathrm{G}_{1}\right.$ only or $\mathrm{CD}-\mathrm{PEI}$ only), which indicated the formation of supramolecular particles by host-guest complexation. The size of the objects showed positive correlation with the ionic strength: the higher the ionic strength, the larger the nanoparticles. The samples at same concentration and ionic strength did not show significantly difference before and after UV-illumination. We also tried to decrease the concentration of the guest moieties by one 
order of magnitude to $[\mathrm{FA}]=0.01 \mathrm{mM}$, meanwhile the ratio between host and guest was kept as $1: 1$, however diluted samples performed similarly to the highly concentrated samples. Same experiments were performed with $\mathrm{FA}_{4} \mathrm{G}_{0}$, and the $\mathrm{FA}_{4} \mathrm{G}_{0}$ series performed no difference from the $\mathrm{FA}_{8} \mathrm{G}_{1}$ samples [Figure $\mathbf{S 5 . 4}$ ].

Compared to the previously reported dendrimer-polymer cross-linking architectures, a monovalent guest stopper was not employed in this study, thus we expected that the nanoparticles would grow continuously in time ${ }^{[8,29,30,42]}$. However, no clear trend in the size evolution of the nanoparticles was observed within 3 hours, in any of the conditions that were investigated. It is possible that at each ionic strength, the host-guest interaction is always in equilibrium against the electrostatic repulsive interactions.

a)

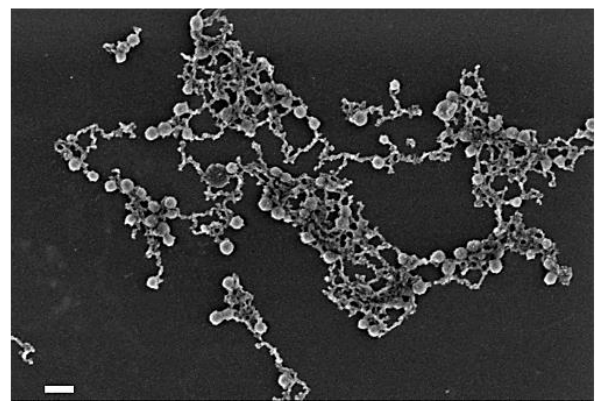

b)

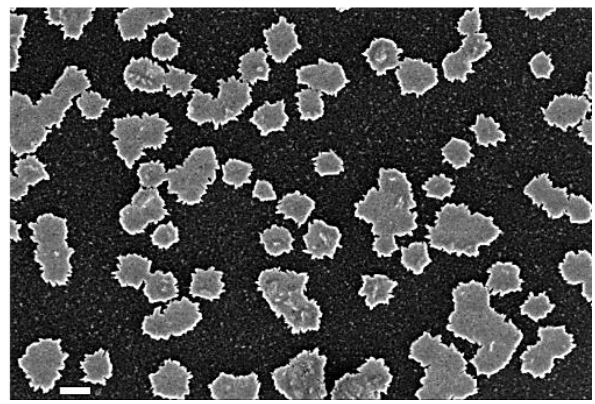

Figure 5.3. SEM image of the bi-component system with $\mathrm{FA}_{8} \mathrm{G}_{1}$ in initial state, a) $[\mathrm{FA}]=0.1 \mathrm{mM},[\mathrm{NaCl}]$ $=0 \mathrm{M}$. b) $[\mathrm{FA}]=0.1 \mathrm{mM},[\mathrm{NaCl}]=0.2 \mathrm{M}$. Scale bar $=200 \mathrm{~nm}$.

The samples were further analyzed by scanning electron microscopy (SEM). In the sample with no ionic strength, spherical particles with a diameter around 70-80 nm were observed, the nanoparticles were connected by fibers [Figure 5.3a]. In sample under $0.2 \mathrm{M}$ $\mathrm{NaCl}$ environment, the particles displayed an irregular shape in the range of 100-500 $\mathrm{nm}$ in diameter, which diameters are in agreement with the DLS analysis [Figure 5.3b]. 
To conclude about the bi-component system, the FA-PAMAM could form supramolecular nanoparticles with CD-PEI polymers in both the initial state, and under illumination with UV light. The observed particle size is related to the ionic strength of the solution, but does not respond to photo-isomerization of the FA moieties. The differences on binding affinity between trans- and cis- FA in $\beta-\mathrm{CD}$ is not significant enough to reach photo-responsive assembly and disassociation.

\subsubsection{A tri-component system}

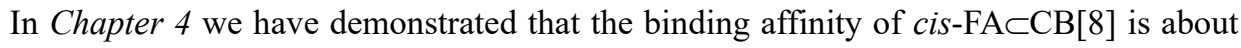
two orders of magnitude higher than the $\mathrm{FA} \subset \beta$-CD binding affinity. In monovalent binding, the $\mathrm{CB}[8]$ cavity could win the competitive complexation of cis-FA against 10 times concentrated $\beta-\mathrm{CD}^{[47]}$. Consequently, $\mathrm{CB}[8]$ was introduced into the system to disrupt the multivalent $F A \subset \beta-C D$ complex.

The concentration of the tri-component system was limited by the solubility of $\mathrm{CB}[8]$ in water (up to $0.05 \mathrm{mM}$ ). First experiment was performed with $\mathrm{FA}_{4} \mathrm{G}_{0}$ as $\mathrm{FA}: \beta-\mathrm{CD}: \mathrm{CB}[8]=$ $1: 1: 1,[\mathrm{FA}]=0.05 \mathrm{mM}$, the sample were measured by DLS at initial state, after UVillumination $(\lambda=365 \mathrm{~nm}, 1 \mathrm{~min})$ and purple light illumination $(\lambda=420 \mathrm{~nm}, 2 \mathrm{~min})$. A comparative experiment was performed as a mixture with $\mathrm{FA}_{4} \mathrm{G}_{0}$ as $\mathrm{FA}: \mathrm{CB}[8]=1: 1$, $[\mathrm{FA}]=$ $0.05 \mathrm{mM}$. This sample was firstly illuminated by UV light, then the CD-PEI was added to the solution at the cis- state, followed by illumination with $420 \mathrm{~nm}$ purple light to induce the cis-to-trans isomerization. According to the UV/Vis absorption spectrum, illumination with $420 \mathrm{~nm}$ purple light cannot yield a complete cis-to-trans isomerization. In both experiments, the DLS signals always appeared at $\sim 80 \mathrm{~nm}$ and showed no responsiveness to either UV or 
purple light illumination [Figure 5.5]. The 1:1:1 series were diluted by 5 times to $[\mathrm{FA}]=0.01$ $\mathrm{mM}$, no particles can be observed by DLS analysis at this concentration- neither in the initial state, nor after illumination with UV light.

a)
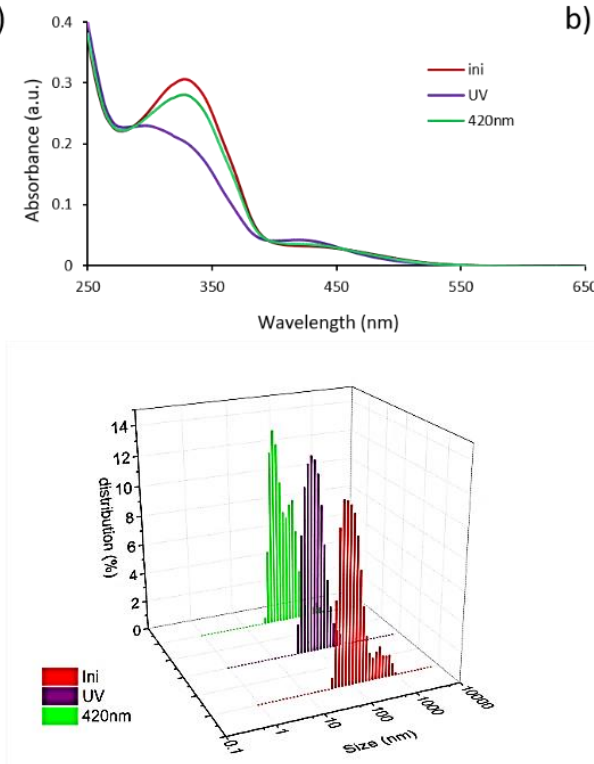

b)
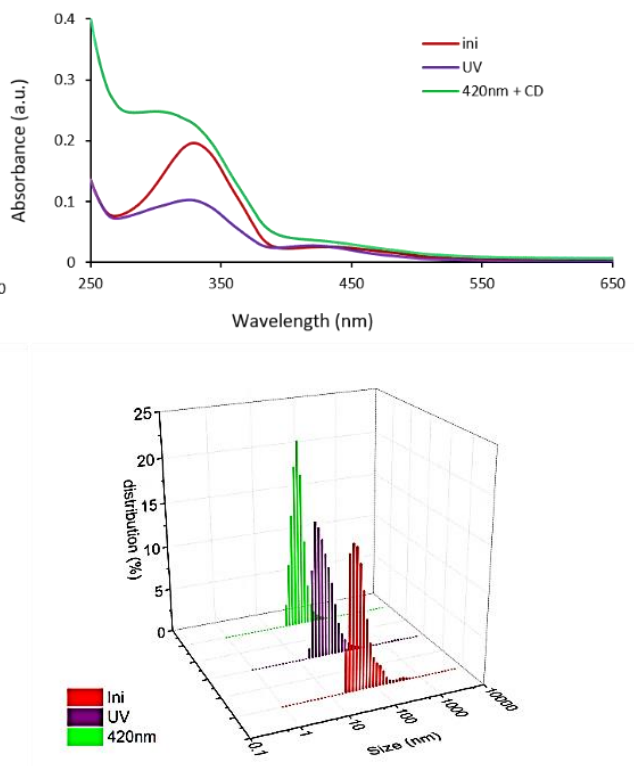

Figure 5.5. UV/Vis absorption spectrum (up) and DLS analysis (down) for FA : CB : CD =1:1:1 with $\mathrm{FA}_{4} \mathrm{G}_{0},[\mathrm{FA}]=0.05 \mathrm{mM}$, at initial state, after UV illumination $(\lambda=365 \mathrm{~nm}, 1 \mathrm{~min})$ and purple illumination ( $\lambda=420 \mathrm{~nm}, 2 \mathrm{~min}$ ), a) all the three components were mixed together from beginning, $b$ ) FA and CB were mixed firstly, CD-PEI were added after UV-illumination.

In order to enhance the competitiveness of the $\mathrm{CB}[8]$ cavity against the $c i s-\mathrm{FA} \subset \beta-\mathrm{CD}$ interaction, the ratio of $\mathrm{CB}[8]$ moiety was increased up to $\mathrm{FA}: \beta-\mathrm{CD}: \mathrm{CB}[8]=1: 1: 10$. Since [CB] was limited to $50 \mu \mathrm{M}$, the FA and CD concentration was set as $5 \mu \mathrm{M}$. This sample is too diluted to have any signal detected by neither UV/Vis absorption spectrum nor DLS analysis. Next, the concentration of FA was increased to $0.01 \mathrm{mM}$, with a ratio as FA : $\beta-\mathrm{CD}$ : $\mathrm{CB}[8]=1: 1: 5$. In the previously reported azobenzene-supramolecular nanoparticles, the 
disassembly occurs after 24 hours of the trans-to-cis isomerization ${ }^{[42]}$, thus we monitored the mixture by DLS up to 24 hours after UV illumination. For both $\mathrm{FA}_{4} \mathrm{G}_{0}$ and $\mathrm{FA}_{8} \mathrm{G}_{1}$ samples, particles up to $100 \mathrm{~nm}$ could always be observed, which indicated that the supramolecular nanoparticles did not show responsiveness to the photo-isomerization process [Figure 5.6].

a)

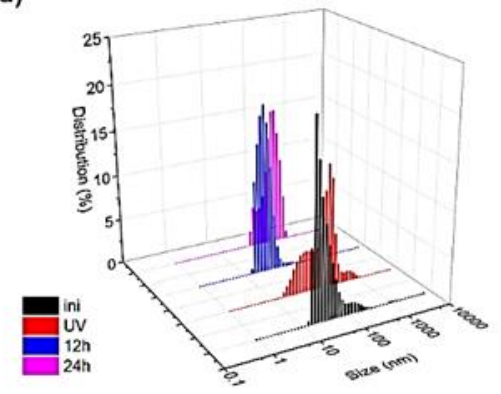

b)

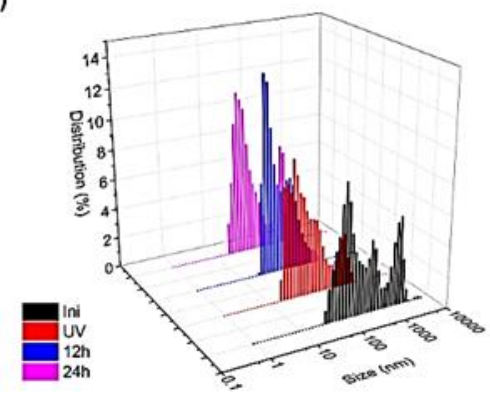

Figure 5.6. DLS analysis for $\mathrm{FA}: \beta-\mathrm{CD}: \mathrm{CB}[8]=1: 1: 5,[\mathrm{FA}]=0.01 \mathrm{mM}$, monitored at initial state, directly after UV illumination, 12 hours and 24 hours after UV illumination. a) $\mathrm{FA}_{4} \mathrm{G}_{0}$, b) $\mathrm{FA}_{8} \mathrm{G}_{1}$.

Job plot was also performed to find a relation between the ratio of FA and $\beta-C D$. The concentration of $\mathrm{CB}[8]$ was set as $0.05 \mathrm{mM}$, and total concentration of $[\mathrm{FA}]$ plus $[\beta-\mathrm{CD}]$ was set as constant as $0.01 \mathrm{mM}$. However, in all conditions, the DLS signal was found as heterogenic peaks from $\sim 30 \mathrm{~nm}$ up to $\sim 1000 \mathrm{~nm}$. No obviously maximum or minimum value can be found according to the ratio $\mathrm{r}=[\mathrm{FA}] /([\mathrm{FA}]+[\mathrm{CD}])$, thus we cannot conclude a stoichiometry between the multivalent FA guest and $\beta-\mathrm{CD}$ host with presence of $\mathrm{CB}[8]$ [Figure S5.5].

Next, we studied samples with $0.1 \mathrm{M} \mathrm{NaCl}$ ionic strength, FA : $\beta-\mathrm{CD}: \mathrm{CB}[8]=1: 1: 1$, $[\mathrm{FA}]=0.01 \mathrm{mM}$. Firstly, the three components were mixed together. In the initial state, DLS experiments evidenced two signals, corresponding to diameters of about $280 \mathrm{~nm}$ and 1500 $\mathrm{nm}$ respectively. After illumination with UV light ( $\lambda=365 \mathrm{~nm}, 1 \mathrm{~min})$, no significant change 
was observed. Then the sample was illuminated with purple light $(\lambda=420 \mathrm{~nm}, 2 \mathrm{~min})$ to perform cis-to-trans isomerization. The main peak on DLS was still found at $\sim 250 \mathrm{~nm}$. A comparative experiment was also performed. At the beginning, there are only the FA and $\mathrm{CB}[8]$ in the mixture, particles with various sizes were also observed, corresponding to the self-aggregation of the FA-PAMAM. This mixture was illuminated with UV light for transto-cis isomerization first. No significant difference was observed between the initial state, and the UV-illuminated state. Then CD-PEI was added, followed by purple illumination ( $\lambda$ $=420 \mathrm{~nm}$ ), which yielded a signal at $1 \mathrm{~nm}$ on DLS, which is likely attributable to the newly added unbinding dispersed CD-PEI polymers [Figure 5.7].

a)
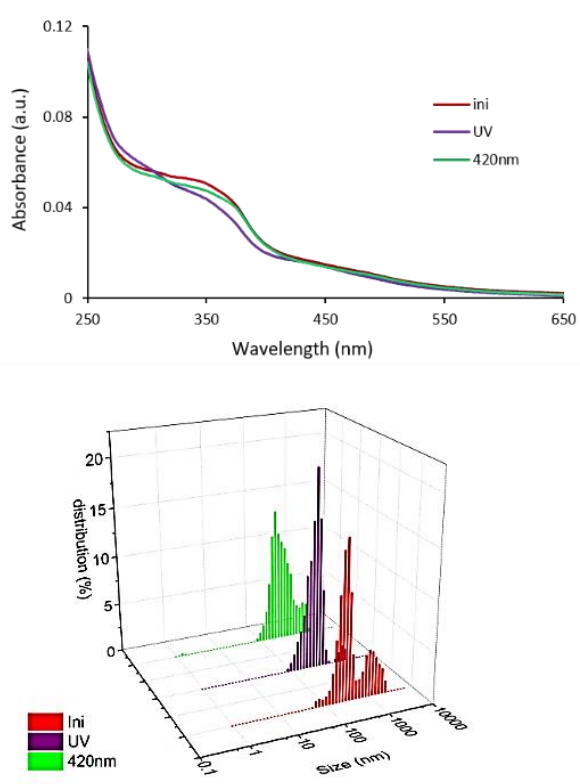

b)
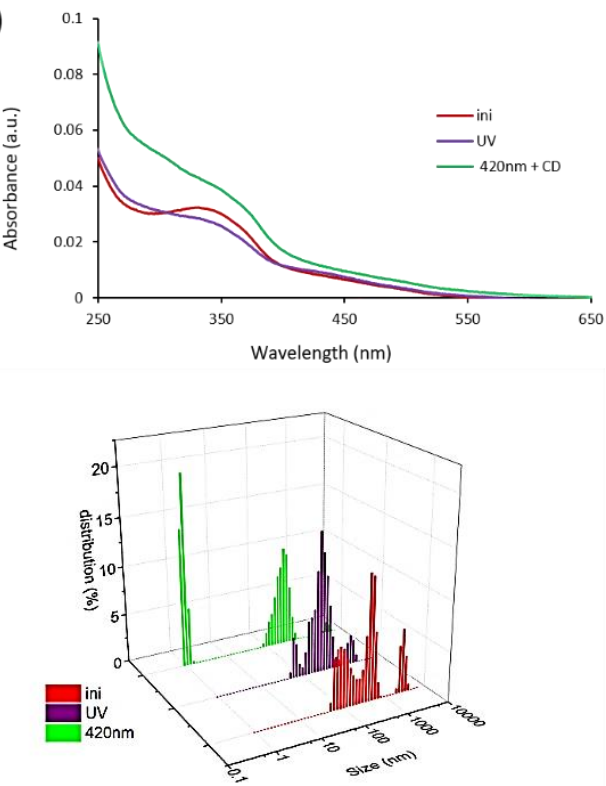

Figure 5.7. UV/Vis absorption spectrum (up) and DLS analysis (down) for FA : $\beta-\mathrm{CD}: \mathrm{CB}[8]=1$ : $1: 1$ with $\mathrm{FA}_{4} \mathrm{G}_{0},[\mathrm{FA}]=0.01 \mathrm{mM},[\mathrm{NaCl}]=0.1 \mathrm{M}$ at initial state, after UV illumination $(\lambda=365 \mathrm{~nm}$, $1 \mathrm{~min}$ ) and purple illumination $(\lambda=420 \mathrm{~nm}, 2 \mathrm{~min})$, a) all the three components were mixed together from the beginning, b) FA and CB were mixed firstly, and CD-PEI was added after UV-illumination. 
We also performed the FA : $\beta-C D: C B[8]=1: 1: 1$ series with $\mathrm{FA}_{8} \mathrm{G}_{1}$. When the three components were mixed together, initially, the sample behaved as $\mathrm{FA}_{4} \mathrm{G}_{0}$. In the comparative experiment, after UV-illumination, a new signal was found at $1 \mathrm{~nm}$ in DLS measurement. The cis- $\mathrm{FA}_{8} \mathrm{G}_{1}$ could partially form host-guest pairs with $\mathrm{CB}[8]$ cavities, and lead to dispersion of the nanoparticles in solution. And the bigger size particle at $400 \mathrm{~nm}$ remains still, possibly the inclusion and disperse were hindered by trans-to-cis conversion ratio and the salt induced self-aggregation of the dendrimers. When CD-PEI was added and the mixture was illuminated with purple light $(\lambda=420 \mathrm{~nm})$, then both the signal at $1 \mathrm{~nm}$ and 400 nm were observed. the small size signal can be classified to FA-PAMAM in CB complex or uncharged CD-PEI or both [Figure 5.8].

a)
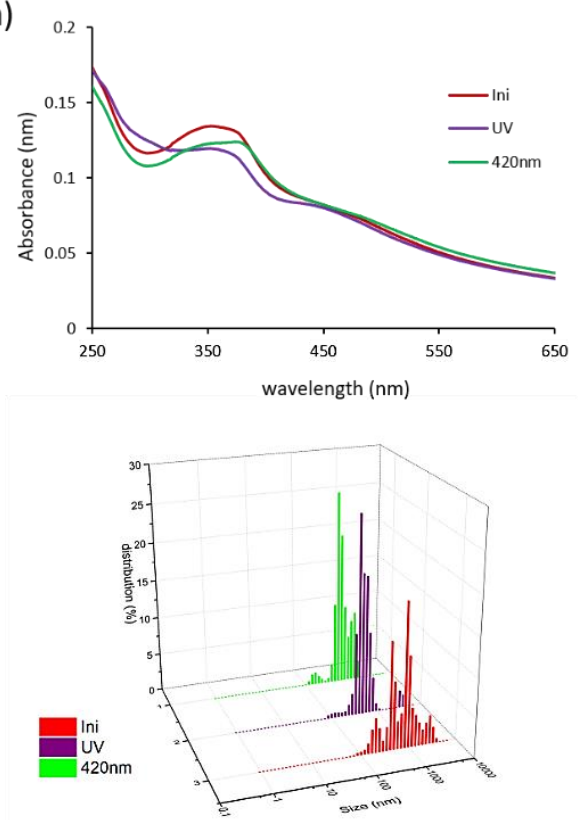

b)
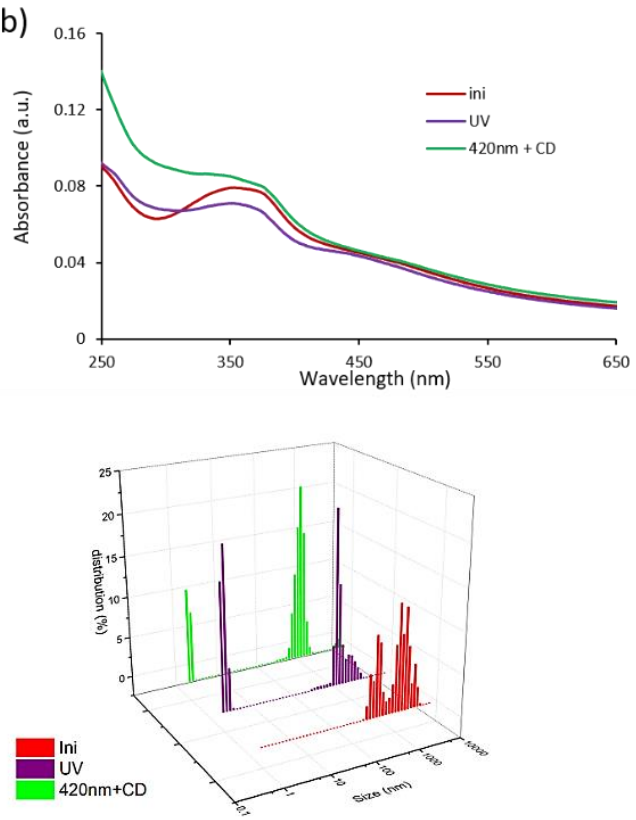

Figure 5.8. UV/Vis absorption spectrum (up) and DLS analysis (down) for FA : CB : CD $=1: 1: 1$ 
with $\mathrm{FA}_{8} \mathrm{G}_{1},[\mathrm{FA}]=0.01 \mathrm{mM},[\mathrm{NaCl}]=0.1 \mathrm{M}$ at initial state after $\mathrm{UV}$ illumination $(\lambda=365 \mathrm{~nm}, 1 \mathrm{~min})$ and purple illumination $(\lambda=420 \mathrm{~nm}, 2 \mathrm{~min})$. a) all the three components were mixed together from beginning, b) FA and CB were mixed firstly, CD-PEI was added after UV-illumination.

When analyzing a multivalent system, one important factor is cooperativity, which performs as the influence of the employed binding site to the un-charged binding sites ${ }^{[48-49]}$. Normally, the cooperativity will not be considered in a synthetic system, i.e. each binding site was considered as individual and showed no influence to other binding sites $^{[50-56]}$.

In the complexation between cis-FA-PAMAM and $\mathrm{CB}[8]$, stepwise equilibria can be written as:

$$
K_{\mathrm{n}}=K_{\mathrm{i}}(m-n+1) / n
$$

where the $m$ is the total binding site of the multivalent guest, $n$ is the number of the charged binding site, $K_{\mathrm{i}}$ is the binding constant of individual host-guest complex ${ }^{[57-58]}$. In the $\mathrm{FA}_{8} \mathrm{G}_{1^{-}}$ $\mathrm{CB}[8]$ complexation, the binding affinity of first binding complex can be calculated as $K_{1}=K_{\mathrm{i}}$ $(8-1+1) / 1=8 K_{\mathrm{i}}$, meanwhile binding affinity of the last binding complex will be $K_{8}=K_{\mathrm{i}}(8-$ $8+1) / 8=1 / 8 K_{\mathrm{i}}$. Overall, the FA-CB[8] interaction in this study was expected with a binding constant between $10^{4} \sim 10^{6} \mathrm{M}^{-1}$ [Figure 5.9].

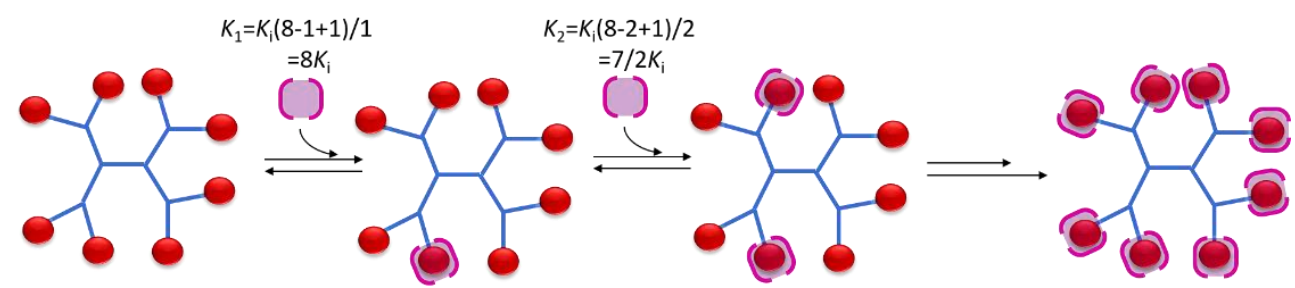

Figure 5.9. Stepwise host-guest binding equilibria of $c i s-\mathrm{FA}_{8} \mathrm{G}_{1}$ and $\mathrm{CB}[8]$

On the other hand, the multivalent interaction between FA-PAMAM and CD-PEI is more complicated. Generally, for a multivalent guest molecule A with $m$ guest binding sites $\mathrm{M}$, and 
a multivalent host molecule $\mathrm{B}$ with $n$ binding sites $\mathrm{N}$, the first step binding works intermolecularly, resulting in complex $[\mathrm{AB}]$ with a single host-guest complex $\mathrm{M} \subset \mathrm{N}$, binding constant is expected as $K_{1}=n \times m \times K_{\mathrm{i}}$. In the next step, there are two possibilities: a) the remained free binding site $\mathrm{M}$ from $\mathrm{A}$ and $\mathrm{N}$ from $\mathrm{B}$ forms intramolecular host-guest binding within the $[\mathrm{AB}]$ complex, forming a closed supramolecular complex [AB]' with multiple $\mathrm{M} \subset \mathrm{N}$ complexes ${ }^{[59-60]}$, or b), the $[\mathrm{AB}]$ complex was binding to another $[\mathrm{A}]$ or $[\mathrm{B}]$ with their free binding sites, resulting in complex $\left[\mathrm{AB}_{2}\right]$ or $\left[\mathrm{A}_{2} \mathrm{~B}\right]$. In practice, both the possibilities occurred in parallel, results in the complex with multiple intermolecular and intra molecular binding $\left[\mathrm{A}_{\mathrm{n}} \mathrm{B}_{\mathrm{m}}\right]$, the overall binding constant presents as

$$
K_{\mathrm{ov}}=K_{\mathrm{i}}\left(K_{\mathrm{i}} \mathrm{EM}\right)^{(\mathrm{n}-1)}
$$

with an empirical factor effective molarity $\mathrm{EM}^{[50,61]}[$ Figure 5.10].

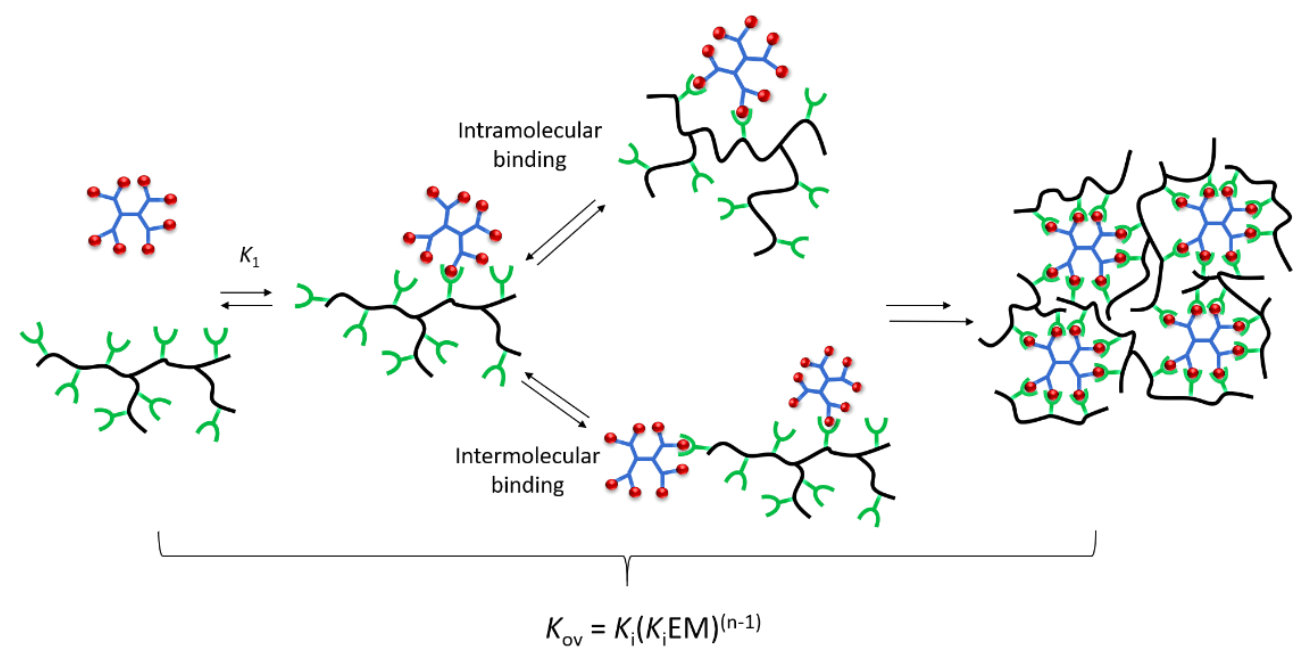

Figure 5.10. Supramolecular binding between multi valent guest $F_{8} \mathrm{G}_{1}$ and multi valent host CD-PEI.

In the complexation between FA-PAMAM and CD-PEI, the first step binding for $\mathrm{FA}_{4} \mathrm{G}_{0}$ 
with CD-PEI was expected with a binding constant $K_{1}=32 K_{\mathrm{i}}$, for $\mathrm{FA}_{8} \mathrm{G}_{1}$, the first step binding was expected $K_{1}=64 K_{\mathrm{i}}$, which already in the same magnitude to the binding constant of $c i s-\mathrm{FA} \subset \mathrm{CB}$ complex. When take the overall binding affinity $K_{\mathrm{ov}}$ into consideration, the exponent factor (n-1) in the equation (2) will be 3 for $\mathrm{FA}_{4} \mathrm{G}_{0}$ and 7 for $\mathrm{FA}_{8} \mathrm{G}_{1}$, which suggested a $K_{\mathrm{ov}}$ up to $10^{12} \mathrm{M}^{-1}$ or even $10^{24} \mathrm{M}^{-1}$ respectively. Then the $K_{\text {FA-CB }}$ about $5^{*} 10^{5} \mathrm{M}^{-1}$ is obviously not strong enough to win the competitive host-guest complexation and to further disturb the multivalent supramolecular nanoparticles.

\subsection{Conclusions}

We synthesized PAMAM dendrimers functionalized with ortho-fluorinated azobenzenes as a multivalent guest, and $\beta$-CD functionalized PEI polymer as a multivalent host. The FA could form host-guest complex with $\beta$-CD in both trans- and cis- state with binding constant in the same order of magnitude. In the FA-PAMAM and CD-PEI bi-component system, nanoobjects were observed by DLS and SEM before and after UV illumination with similar size, however did not show any responsiveness to photo-isomerization process. Then the $\mathrm{CB}[8]$ was introduced to the system to binding with cis-FA, in order to disassemble the supramolecular nanoparticles by competitive complexation. However, the binding between FA-PAMAM and CD-PEI was strongly enhanced by multivalency, thus $\mathrm{CB}[8]$ could not compete against $\beta-\mathrm{CD}$ in the host guest complexation, thus the supramolecular system remained non-photo-responsive. 


\subsection{Acknowledgments}

Prof. Jurriaan Huskens is gratefully acknowledged for discussions on multivalency. Mark Smithers is gratefully acknowledged for performing the SEM measurements.

\subsection{Supporting information}

\subsubsection{Materials and methods}

$\mathrm{CB}[8]$ was purchased from Strem Chemicals, poly(ethylene imine) was purchased from Polyscience Inc., all other chemicals were purchased from Sigma-Aldrich. All chemicals were used as received. The synthesized products were analyzed by ${ }^{1} \mathrm{H}-\mathrm{NMR}$ on a Bruker 400 $\mathrm{MHz}$ NMR spectrometer, all chemical shifts were referenced to the internal $\mathrm{CDCl}_{3}$ signal at $7.24 \mathrm{ppm}, \mathrm{DMSO}-d_{6}$ signal at $2.50 \mathrm{ppm}$ and $\mathrm{D}_{2} \mathrm{O}$ signal at $4.80 \mathrm{ppm}$. Mass spectrum was recorded by a LCT mass spectrometer. Dynamic light scattering analysis (DLS) was carried out on a Nanotrac by Anaspec operating with a Microtrac FLEX Operation Software. UV/Vis absorption spectra were recorded in a conventional quartz cell in a Perkin Elmer UV/Vis Lambda 850 spectrometer. A Hönle bluepoint LED lamp was employed as UV light source $(\lambda=365 \mathrm{~nm})$. A Thorlabs M420L2 lamp was employed as purple light source $(\lambda=420 \mathrm{~nm})$. Scanning electron microscope image was recorded by a Carl-Zeiss 1500 high resolution scanning electron microscope (HR-SEM). 


\subsubsection{Synthesis of the building blocks}

\subsubsection{Synthesis of FA-dendrimers}

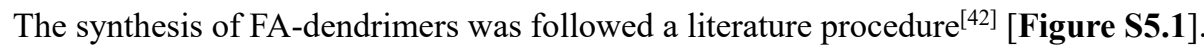<smiles>COC(=O)CCCCOc1cc(F)c(N=Nc2c(F)cccc2F)c(F)c1</smiles>
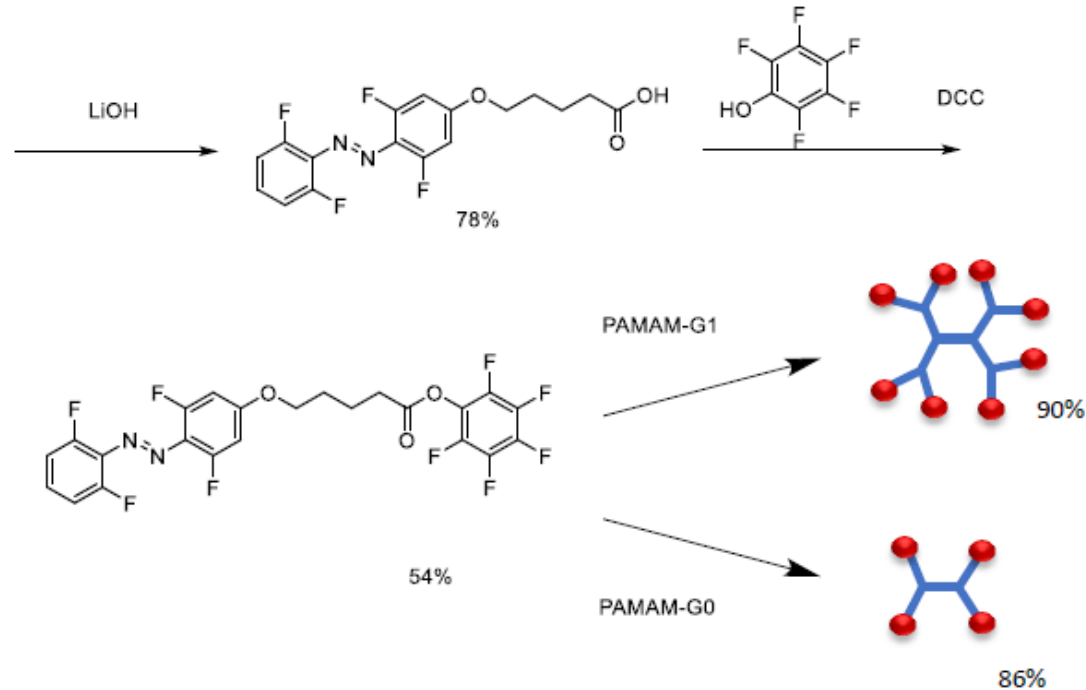

Figure S5.1. Synthesis of FA-PAMAM dendrimer

methyl-5-(4-((2,6-difluorophenyl)diazenyl)-3,5-difluorophenoxy)pentanoate<smiles>COC(=O)CCCCOc1cc(F)c(N=Nc2c(F)cccc2F)c(F)c1</smiles> 
To a solution of methyl 5-bromovalerate in $15 \mathrm{ml}$ of acetonitrile $(0.200 \mathrm{~g}, 1.02 \mathrm{mmol}, 1$ eq.) was added $\mathrm{K}_{2} \mathrm{CO}_{3}(1.410 \mathrm{~g}, 10.2 \mathrm{mmol}, 10$ eq. $)$ and the suspension was stirred for $30 \mathrm{~min}$ at $80^{\circ} \mathrm{C}$. After that time 4-((2,6-difluorophenyl)diazenyl)-3,5-difluorophenol was added (0.277 $\mathrm{g}, 1.02 \mathrm{mmol}, 1$ eq.). Stirring was continued at $80^{\circ} \mathrm{C}$ for 18 hours, followed by removal of the solvent. The residue was dissolved in $30 \mathrm{ml}$ of $\mathrm{CHCl}_{3}$ and extracted 3 times with distilled water $(20 \mathrm{ml})$. The organic layer was separated and dried over $\mathrm{MgSO}_{4}$. The solvent was evaporated and the residue was subjected to silica gel column chromatography $\left(\mathrm{CHCl}_{3}\right)$. The product was obtained as bright orange solid, collected $0.353 \mathrm{~g}$, yield $90 \%$.

${ }^{1} \mathrm{H}$ NMR $\left(400 \mathrm{MHz}, \mathrm{CDCl}_{3}\right): \delta=1.85(\mathrm{~m}, 4 \mathrm{H}), 2.42(\mathrm{t}, J=6.9 \mathrm{~Hz}, 2 \mathrm{H}), 3.69(\mathrm{~s}, 3 \mathrm{H}), 4.03(\mathrm{t}$, $J=5.8 \mathrm{~Hz}, 2 \mathrm{H}), 6.58(\mathrm{~d}, J=11.1 \mathrm{~Hz}, 2 \mathrm{H}), 7.03(\mathrm{t}, \mathrm{J}=8.6 \mathrm{~Hz}, 2 \mathrm{H}), 7.30 \mathrm{ppm}(\mathrm{m}, 1 \mathrm{H})$.

\section{5-(4-((2,6-difluorophenyl)diazenyl)-3,5-difluorophenoxy)pentanoic acid}<smiles>O=C(O)CCCCOc1cc(F)c(N=Nc2c(F)cccc2F)c(F)c1</smiles>

To a stirred solution of methyl-5-(4-((2,6-difluoro-phenyl)diazenyl)-3,5-difluorophenoxy)pentanoate $(0.319 \mathrm{~g}, 0.83 \mathrm{mmol} 1$ eq. $)$ in $6 \mathrm{ml}$ of $\mathrm{MeOH} / \mathrm{THF}(1: 1)$ was added $\mathrm{LiOH}(0.199$ $\mathrm{g}, 8.3 \mathrm{mmol}, 10$ eq.) dissolved in $1.55 \mathrm{ml}$ distilled water. The reaction was stirred at room temperature for $3 \mathrm{~h}$. After that time the solution was acidified with $1 \mathrm{M}$ hydrochloric acid $(\mathrm{pH}=1)$ followed by dilution with $50 \mathrm{ml}$ of $\mathrm{CHCl}_{3}$. The suspension was extracted 3 times with $30 \mathrm{ml}$ of distilled water. The organic layer was dried over $\mathrm{MgSO}_{4}$ and the solvent was 
removed under reduced pressure. The product was obtained as brown-orange solid and was used without further purification, collected $0.240 \mathrm{~g}$, yield $78 \%$.

${ }^{1} \mathrm{H}$ NMR (400MHz, DMSO- $\left.d_{6}\right): \delta=1.64(\mathrm{~m}, 2 \mathrm{H}), 1.74(\mathrm{~m}, 2 \mathrm{H}), 2.29(\mathrm{t}, J=7.3 \mathrm{~Hz}, 2 \mathrm{H})$, $4.13(\mathrm{t}, J=6.4 \mathrm{~Hz}, 2 \mathrm{H}), 7.00(\mathrm{~d}, J=11.9 \mathrm{~Hz}, 2 \mathrm{H}), 7.31(\mathrm{t}, J=8.9 \mathrm{~Hz}, 2 \mathrm{H}), 7.55(\mathrm{tt}, J=8.4$, 6.1 Hz, 1H), 12.09 ppm (br, 1H).

perfluorophenyl-5-(4-((2,6-difluorophenyl)diazenyl)-3,5-difluorophenoxy)pentanoate<smiles>O=C(CCCCOc1cc(F)c(N=Nc2c(F)cccc2F)c(F)c1)Oc1c(F)c(F)c(F)c(F)c1F</smiles>

To a solution of 5-(4-((2,6-difluorophenyl)-diazenyl)-3,5-difluorophenoxy)pentanoic acid (0.292 g, $0.79 \mathrm{mmol}, 1$ eq.) in $10 \mathrm{ml}$ of THF was added dicylohexylcarbodiimide $(0.243 \mathrm{~g}$, $1.18 \mathrm{mmol}, 1.5$ eq.) and the mixture was stirred for $30 \mathrm{~min}$ at room temperature. After adding pentafluorophenol (0.217 g, $1.18 \mathrm{mmol}, 1.5$ eq.) stirring was continued for $18 \mathrm{~h}$. The suspension was filtered and the solvent was removed under reduced pressure. The residue was subjected to silica gel column chromatography $\left(\mathrm{CHCl}_{3}\right)$. After removal of the solvent the product was obtained as a bright orange solid, collected $0.229 \mathrm{~g}$, yield $54 \%$.

${ }^{1} \mathrm{H}$ NMR (400MHz, $\left.\mathrm{CDCl}_{3}\right): \delta=1.98(\mathrm{~m}, 4 \mathrm{H}), 2.79(\mathrm{t}, J=6.8 \mathrm{~Hz}, 2 \mathrm{H}), 4.08(\mathrm{t}, J=5.8 \mathrm{~Hz}$, 2H), $6.60(\mathrm{~d}, J=11.0 \mathrm{~Hz}, 2 \mathrm{H}), 7.01(\mathrm{t}, J=8.6 \mathrm{~Hz}, 2 \mathrm{H}), 7.31 \mathrm{ppm}(\mathrm{m}, 1 \mathrm{H})$. 
Octa- 5-(4-((2,6-difluorophenyl)diazenyl)-3,5-difluorophenoxy)-pentanoic-acidpolyamidoamine-dendrimer (Generation 1) $\left(\mathbf{F A} \mathbf{A}_{8} \mathbf{G}_{1}\right)$<smiles>CC(C)C(=O)CCCCCOc1cc(F)c(N=Nc2c(F)cccc2F)c(F)c1</smiles>

To a solution of perfluorophenyl-5-(4-((2,6-difluorophenyl)diazenyl)-3,5-difluorophenoxy)pentanoate (0.078 g, $0.145 \mathrm{mmol}, 16$ eq.) in a $1: 1$ mixture of $2.5 \mathrm{ml}$ of $\mathrm{CH}_{2} \mathrm{Cl}_{2}$ and DMF (1: 1) was added PAMAM dendrimer generation $1(0.011 \mathrm{~g}, 0,009 \mathrm{mmol}, 1 \mathrm{eq}$.$) in 0.25 \mathrm{ml}$ of DMF solution. The reaction was stirred under an atmosphere of argon for $18 \mathrm{~h}$. The resulting mixture was precipitated with $\mathrm{Et}_{2} \mathrm{O}$, and collected by centrifugation, yielding an orange solid, collected $0.034 \mathrm{~g}$, yield $90 \%$.

${ }^{1} \mathrm{H}$ NMR (400MHz, DMSO- $\left.d_{6}\right): \delta=1.66(\mathrm{~m}, 32 \mathrm{H}), 2.18(\mathrm{~m}, 40 \mathrm{H}), 2.42(\mathrm{~m}, 12 \mathrm{H}), 2.64(\mathrm{~m}$, 24H), $3.08(\mathrm{~m}, 40 \mathrm{H}), 4.09(\mathrm{~m}, 16 \mathrm{H}), 6.94(\mathrm{~d}, J=12.5 \mathrm{~Hz}, 16 \mathrm{H}), 7.29(\mathrm{t}, J=9.2 \mathrm{~Hz}, 16 \mathrm{H})$, $7.53(\mathrm{~m}, 8 \mathrm{H}), 7.92 \mathrm{ppm}(\mathrm{m}, 20 \mathrm{H})$

TOF MS ES+ $(\mathrm{M}+2 \mathrm{H})$ Calculated for $\left[\mathrm{C}_{198} \mathrm{H}_{226} \mathrm{~N}_{42} \mathrm{O}_{28} \mathrm{~F}_{32}{ }^{2+}\right]$ : 2123.85, found: 2124.07 .

Tetra- 5-(4-((2,6-difluorophenyl)diazenyl)-3,5-difluorophenoxy)-pentanoic-acidpolyamidoamine-dendrimer (Generation 0) $\left(\mathbf{F A}_{4} \mathbf{G}_{0}\right)$<smiles>COCCCCC(C)C(=O)CCCCOc1cc(F)c(N=Nc2c(F)cccc2F)c(F)c1</smiles> 
To a solution of perfluorophenyl-5-(4-((2,6-difluorophenyl)diazenyl)-3,5-difluorophenoxy)pentanoate (0.246 g, $0.427 \mathrm{mmol}, 8$ eq.) in a 1:1 mixture of $2.5 \mathrm{ml}$ of $\mathrm{CH}_{2} \mathrm{Cl}_{2}$ and DMF (1:1) was added PAMAM dendrimer generation 0 (0.028 g, 0,053 mmol, 1 eq.) in $1 \mathrm{ml}$ of DMF solution. The reaction was stirred under an atmosphere of argon for $18 \mathrm{~h}$. The resulting mixture was precipitated with $\mathrm{Et}_{2} \mathrm{O}$, and collected by centrifugation, yielding an orange solid, collected $0.088 \mathrm{~g}$, yield $86 \%$.

${ }^{1} \mathrm{H}$ NMR (400MHz, DMSO- $\left.d_{6}\right): \delta=1.66(\mathrm{~m}, 16 \mathrm{H}), 2.18(\mathrm{~m}, 20 \mathrm{H}), 2.42(\mathrm{~m}, 8 \mathrm{H}), 2.64(\mathrm{~m}, 4 \mathrm{H})$, $3.08(\mathrm{~m}, 12 \mathrm{H}), 4.09(\mathrm{~m}, 8 \mathrm{H}), 6.94(\mathrm{~d}, J=12.5 \mathrm{~Hz}, 8 \mathrm{H}), 7.29(\mathrm{t}, J=9.2 \mathrm{~Hz}, 8 \mathrm{H}), 7.53(\mathrm{~m}$, 4H), $7.92 \mathrm{ppm}(\mathrm{m}, 8 \mathrm{H})$.

TOF MS ES+ $(\mathrm{M}+\mathrm{H})$ Calculated for $\left[\mathrm{C}_{90} \mathrm{H}_{97} \mathrm{~N}_{18} \mathrm{O}_{12} \mathrm{~F}_{16}{ }^{+}\right]$: 1925.73, found: 1925.74 .

\subsubsection{Synthesis of CD-PEI polymer}
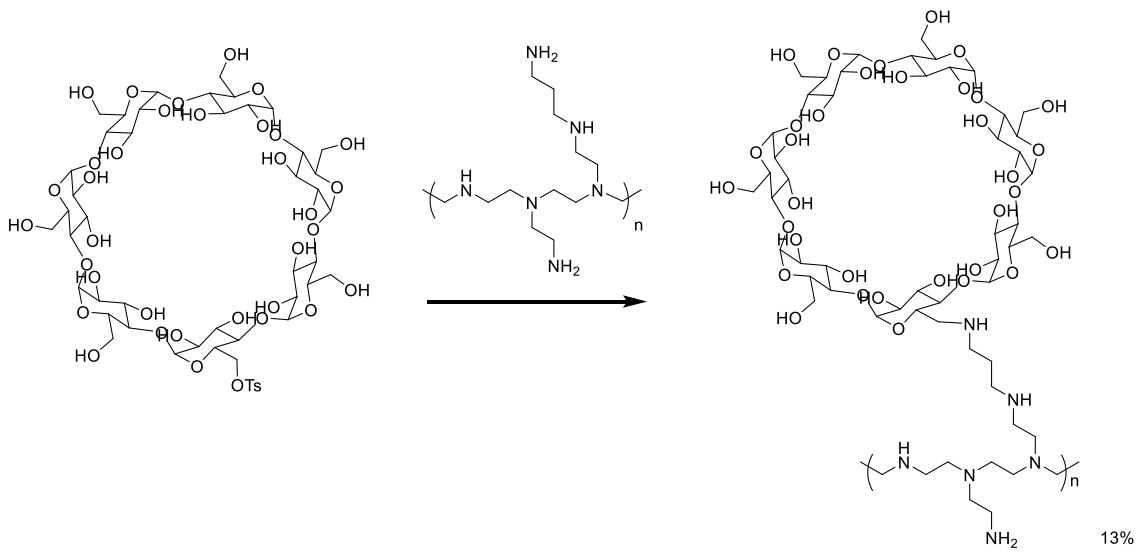

Figure S5.2. Synthesis of CD-PEI. 
The preparation of the $\beta$-CD-functionalized PEI polymer was based on a literature procedure $^{[29]}$ [Figure S5.2]. To a solution of branched poly(ethylene imine) $[\mathrm{Mw}=10000 \mathrm{~g}$ $\left.\mathrm{mol}^{-1}\right](0.115 \mathrm{~g}, 0.0115 \mathrm{mmol})$ dissolved in $21 \mathrm{ml}$ of DMSO under argon at $60^{\circ} \mathrm{C}$, a solution of 6-monotosyl- $\beta$-cyclodextrin $(0.644 \mathrm{~g}, 0.506 \mathrm{mmol})$ and $0.345 \mathrm{ml}$ triethylamine in $16 \mathrm{ml}$ of DMSO was added slowly under argon by using a syringe while stirring. The resulting solution was stirred at $60{ }^{\circ} \mathrm{C}$ for three days under argon. The solution was cooled to room temperature and diluted with $40 \mathrm{~mL}$ deionized water with a resulting $\mathrm{pH}=10$. The solution was transferred to a Spectra/Por MWCO 6-8k D membrane and dialyzed against water for 4 days. The dialyzed solutions were filtered over paper and lyophilized to afford $189 \mathrm{mg}$ of a fluffy, near-white solid.

${ }^{1} \mathrm{H}$ NMR (400 MHz, D $\left.2 \mathrm{O}\right): \delta=2.2-3.5(\mathrm{br}, 115.6 \mathrm{H}), 3.56-3.87(\mathrm{~m}, 42.1 \mathrm{H}), 5.05-5.39 \mathrm{ppm}$ (br, 7H). The ${ }^{1} \mathrm{H}$ NMR indicated that the ratio between $\mathrm{CD}$ unit and ethylene imine unit is about $1: 21,8 \beta$-CD units per each PEI polymer in average.

\subsubsection{UV/Vis spectrum for $F A_{8} G_{1}$ CD-PEI bi-component system}

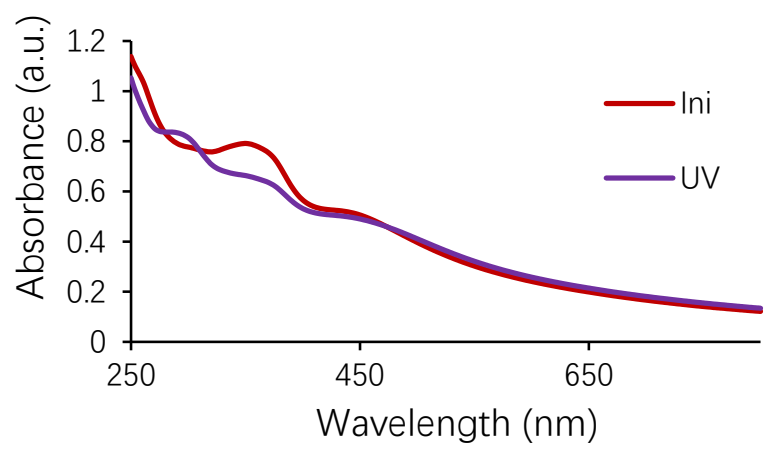

Figure S5.3. UV/Vis spectrum for sample $\mathrm{FA}_{8} \mathrm{G}_{1}$ with $\mathrm{CD}-\mathrm{PEI},[\mathrm{FA}]=0.1 \mathrm{mM},[\mathrm{NaCl}]=0.2 \mathrm{M}$. UV light: $\lambda=365 \mathrm{~nm}$, illumination time: $1 \mathrm{~min}$. 


\subsubsection{DLS data for $\mathrm{FA}_{4} \mathrm{G}_{0}$ CD-PEI bi-component system}

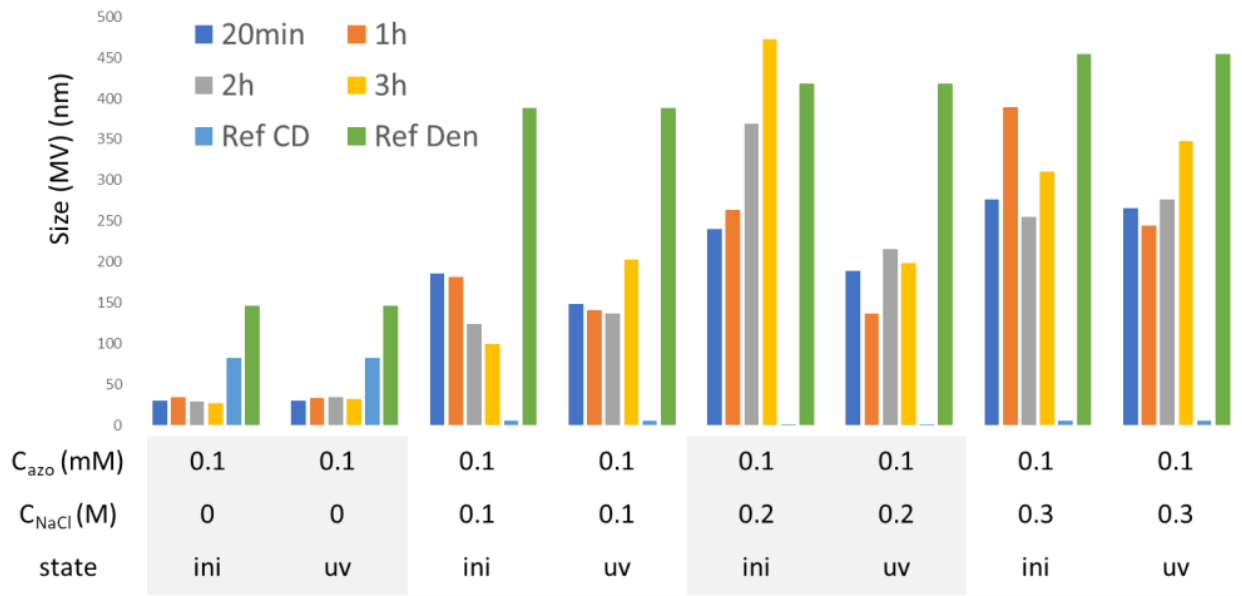

Figure S5.4. DLS analysis for $\mathrm{FA}_{8} \mathrm{G}_{1} \mathrm{CD}-\mathrm{PEI}$ di component system.

\subsubsection{Job plot for $\mathrm{FA}_{4} \mathrm{G}_{0}, \mathrm{CD}, \mathrm{CB}$ tri-component system}

a)

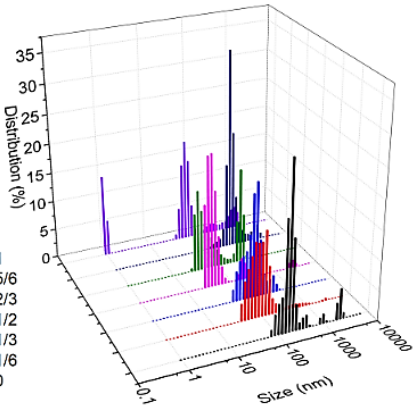

b)

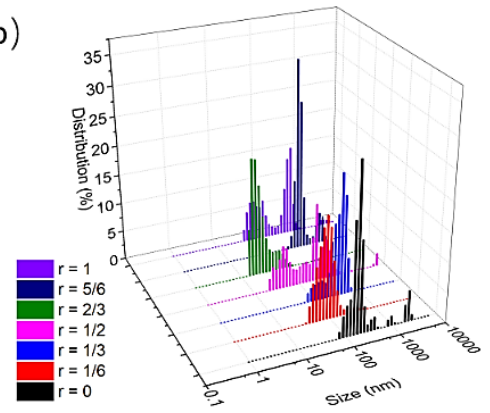

Figure S5.5. Job plot for the $\mathrm{FA}_{4} \mathrm{G}_{0}, \mathrm{CD}, \mathrm{CB}$ tri-component system, $[\mathrm{CB}]=0.05 \mathrm{mM},[\mathrm{FA}]+[\mathrm{CD}]=$ $0.01 \mathrm{mM}, \mathrm{r}=[\mathrm{FA}] /([\mathrm{FA}]+[\mathrm{CD}]), \mathrm{a})$ as initial state, $\mathrm{b})$ after UV illumination. 


\subsection{References}

[1] J-M Lehn, Proc. Nat. Acad. Sci. USA, 2002, 99, 4763.

[2] G. M. Whitesides, M. Boncheva, Proc. Nat. Acad. Sci. USA, 2002, 99, 4769.

[3] J-M. Lehn, Supramolecular chemistry: concepts and perspectives, VCH, New York, 1995.

[4] J. L. Atwood, J. E. D. Davies, D. D. MacNicol, F. Vögtle, J-M. Lehn, Comprehensive Supramolecular Chemistry, Pergamon, Oxford, 1995.

[5] E. P. Kyba, R. C. Helgeson, K. Madan, G. W. Gokel, T. L. Tarnowski, S. S. Moore, D. J. Cram, J. Am. Chem. Soc., 1977, 99, 2564.

[6] D. J. Cram, Angew. Chem. Int. Ed. Engl., 1986, 25, 1039.

[7] M. Auffan, J. Rose, J-Y. Bottero, G. V. Lowry, J-P. Jolivert, M. R. Wiesner, Nat. Nanotechol., 2009, 4, 634.

[8] H. Wang, S. Wang, H. Su, K-J. Chen, A. L. Armijo, W-Y. Lin, Y. Wang, J. Sun, K. Kamei, J. Czernin, C. G. Radu, H-R. Tseng, Angew. Chem. Int. Ed., 2009, 48, 4344.

[9] M. E. Davis, J. E. Zuckerman, C. H. J. Choi, D. Seligson, A. Tolcher, C. A. Alabi, Y. Yen, J. D. Heidel, A. Ribas, Nature, 2010, 464, 1064.

[10] L. Y. Liu, B. He, Y. Li, G. Wang, S. Chang, Z. Gu. Int. J. Nanomed., 2012, 7, 5249.

[11] G. Wenz, B. H. Han, A. Muller, Chem. Rev., 2006, 106, 782.

[12] B. J. Ravoo, J-C Jacquier, Macromolecules, 2002, 35, 6412.

[13] A. Harada, Y. Takashima, M. Nakahata, Acc. Chem. Res., 2014, 47, 2128.

[14] L. Graña-Suárez, W. Verboom, J. Huskens, Chem. Commun., 2016, 52, 2597.

[15] J. Voskuhl, M. Waller, S. Bandaru, B. A. Tkachenko, C. Fregonese, B. Wibbeling, P. R. Schreiner, B. J. Ravoo, Org. Biomol. Chem., 2012, 10, 4524.

[16] M. V. Reckharsky, Y. Inoue, Chem. Rev., 1998, 98, 1875.

[17] A. Harada, R. Kobayashi, Y. Takashima, A. Hashidzume, H. Yamaguchi, Nat. Chem., 2011, 3, 34 .

[18] M. R. de Jong, J. Huskens, D. N. Reinhoudt, Chem. Eur. J., 2001, 7, 4164.

[19] M. Mammen, S-K. Choi, G. M. Whitesides, Angew. Chem. Int. Ed., 1998, 37, 2754.

[20] J. E. Gestwick, L. E. Strong, L. L. Kiessling, Chem. Biol., 2000, 7, 583.

[21] S. Akai, Y. Kajihara, Y. Nagashima, M. Kamei, J. Arai, M. Bito, J. Sato, J. Carbohydr. Chem., 2001, 20, 121.

[22] J. M. Gargano, T. Ngo, J. Y. Kim, D. W. K. Acheson, W. J. Lees, J. Am. Chem. Soc., 2001, 123, 12909. 
[23] A. Mulder, T. Auletta, A. Sartori, S. Del Ciotto, A. Casnati, R. Ungaro, J. Huskens, D. N. Reinhoudt, J. Am. Chem. Soc., 2004, 126, 6627.

[24] K-J. Chen, S. M. Wolahan, H. Wang, C-H. Hsu, H-W. Chang, A. Durazo, L-P. Hwang, M. A. Garcia, Z. K. Jiang, L. Wu, Y-Y. Lin, H-R. Tseng, Biomaterials, 2011, 32, 2160.

[25] H. Wang, K-J. Chen, S. Wang, M. Ohashi, K. Kamei, J. Sun, J. H. Ha, K. Liu, H-R. Tseng, Chem. Commun., 2010, 46, 1851.

[26] H. Wang, K. Liu, K-J. Chen, Y. Lu, S. Wang, W-Y Lin, F. Guo, K. Kamei, Y-C. Chen, M. Ohashi, M. Wang, M. A. Garcia, X-Z. Zhao, C. K-F. Shen, H-R. Tseng, ACS Nano, 2010, 4, 6235 .

[27] Y. Liu, H. Wang, K. Kamei, M. Yan, K-J. Chen, Q. Yuan, L. Shi, Y. Lu, H-R. Tseng, Angew. Chem. Int. Ed., 2011, 50, 3058.

[28] J. Peng, M. A. Garcia, J. Choi, L. Zhao, J. R. Bernstein, P. Peyda, Y-S. Hsiao, K. W. Liu, W-Y Lin, A. D. Pyle, H. Wang, S. Hou, H-R. Tseng, ACS Nano, 2014, 8, 4621.

[29] R. Meijia-Ariza, G. A. Kronig, J. Huskens, Beilstein J. Org. Chem., 2015, 11, 2388-2399. [30] C. Stoffelen, J. Huskens, Chem. Commun., 2013, 49, 6740.

[31] M-M Russew, S. Hecht, Adv. Mater, 2010, 22, 3348.

[32] B. L. Feringa, W. R. Browne, Molecular Switches, Second Edition, Wiley-VCH: Weinheim, 2011.

[33] H. M. D. Bandara, S. C. Burdette, Chem. Soc. Rev., 2012, 41, 1809.

[34] D-H. Qu, Q-C. Wang, Q-W. Zhang, X. Ma, H. Tian, Chem. Rev., 2015, 115, 7543.

[35] A. Ueno, K. Takahashi, T. Osa, J. Chem. Soc. Chem. Comm., 1980, 17, 837.

[36] Y. Liu, C. Yu, H. Jin, B, Jiang, X. Zhu, Y. Zhou, Z. Lu, D. Yan, J. Am. Chem. Soc., 2013, $135,4765$.

[37] P. Wan, Y. Wang, Y. Jiang. H. Xu, X. Zhang, Adv. Mater., 2009, 21, 4362.

[38] O. Roling, L. Stricker, J. Voskuhl, S. Lamping, B. J. Ravoo, Chem. Commun., 2016, 52, 1964.

[39] K. Iwaso, Y. Takashima, A. Harada, Nat. Chem., 2016, 8, 625.

[40] F. Tian, D. Jiao, F. Biedermann, O. A. Scherman, Nat. Commun., 2012, 3, 1207.

[41] J. del Barrio, P. N. Horton, D. Lairez, G. O. Lloyd, C. Toprakcioglu, O. A. Scherman, J. Am. Chem. Soc., 2013, 135, 11760.

[42] C. Stoffelen, J. Voskuhl, P. Jonkheijm, J. Huskens, Angew. Chem. Int. Ed., 2014, 53, 3400 .

[43] S. A. P. van Rossum, M. Tena-Solsona, J. H. van Esch, R. Eelkema, J. Boekhoven. Chem. Soc. Rev., 2017, 46, 5519. 
[44] D. Bléger, J.Schwarz, A. M. Brouwer, S. Hecht, J. Am. Chem. Soc., 2012, 134, 20597.

[45] C. Knie M. Utecht, F. Zhao, H. Kulla, S. Kovalenko, A. M. Brouwer, S. Hecht, D. Bléger. Chem. Eur. J., 2016, 20, 16492.

[46] L. Zhang, H. Zhang, F. Gao, H. Peng, Y. Xu, W. Weng, RSC Adv., 2015, 5, 12007.

[47] H. Huang, A. Juan, N. Katsonis, J. Huskens, Tetrahedron, 2017, 73, 4913.

[48] C. A. Hunter, H. L. Anderson, Angew. Chem. Int. Ed., 2009, 48, 7488.

[49] G. Ercolani, L. Schiaffino, Angew. Chem. Int. Ed., 2011, 50, 1762.

[50] A. Mulder, J. Huskens, D. N. Reinhoudt, Org. Biomol. Chem., 2004, 2, 3409.

[51] J. Rao, J. Lahiri, R. M. Weis, G. M. Whitesides, J. Am. Chem. Soc., 2000, 122, 2698.

[52] E. Fan, Z. Zhang, W. E. Minke, Z. Hou, C. L. M. J. Verlinde, W. G. J. Hol, J. Am. Chem. Soc., 2000, 122, 2663.

[53] E. A. Merrit, Z. Zhang, J. C. Pickens, M. Ahn, W. G. J. Hol, E. J. Fan, J. Am. Chem. Soc., 2002, 124, 8818.

[54] Z. Zhang, E. A. Merrit, M. Ahn, C. Roach, Z. Hou, C. L. M. J. Verlinde, W. G. Hol, E. J. Fan, J. Am. Chem. Soc., 2002, 124, 12991.

[55] P. I. Kitov, D. R. Bundle, J. Am. Chem. Soc., 2003, 125, 16271.

[56] J. M. Gargano, T. Ngo, J. Y. Kim, D. W. K. Acheson, W. J. Lees, J. Am. Chem. Soc., 2001, 123, 12909.

[57] G. Ercolani, J. Am. Chem. Soc., 2003, 125, 16097.

[58] G. Ercolani, C. Piguet, M. Borkovec, J. Hamacek, J. Phys. Chem. B, 2007, 111, 12195.

[59] W. Kuhn, Kolloidn Zh., 1934, 68, 2.

[60] H. Jacobsen, W. H. Stockmayer, Chem. Phys., 1950, 18, 1600.

[61] L. Mandolini, Adv. Phys. Org. Chem., 1986, 22, 1. 


\section{Chapter 6}

\section{Light-induced self-assembly of nanoplatelets into supra-particular ribbons}

Nanoplatelets of semi-conductors CdSe are promising building blocks for optical display devices, in view of their sharp emission band and low threshold lasing. It is known that these nanoplatelets can aggregate face-to-face to form long-stacking ribbons upon anti-solvent precipitation or solvent evaporation. It is also known that azobenzene photoswitches as photo-responsive ligands can drive the photo-triggered self-assembly of colloidal nanoparticles into aggregates, because their cis-form promotes the establishment of dipoledipole interaction. Here, we combine these two systems by grafting azobenzene photoswitches covalently on CdSe nanoplatelets, and demonstrate the light-fueled self-assembly of nanoplatelets into supra-particular ribbons.

The current chapter will be the part of the future publication Y. Flores, H. Huang, R. Plamont, B. Fleury, N. Katsonis, Photo-responsive Self-assembly of anisotropic hybrid nano-objects. Manuscript in preparation. 


\subsection{Introduction}

Colloidal inorganic nanoparticles are characterized by a high specific surface area and size-dependent properties in terms of catalytic activity, cargo loading, electronic, magnetic, and optical responses ${ }^{[1-5]}$. Self-assembling such nanoparticles into highly ordered supraparticular architectures would allow amplifying their specific properties across multiple length scales ${ }^{[6,7]}$. However, their assembly or dispersion is typically a thermodynamic nonequilibrium process and results in a steady state ${ }^{[8-12]}$. A challenge remaining here is creating smart materials based on such nanoparticles, which could be easily driven away from the steady state and could thus display stimuli-responsive assembly or dispersion.

The light-controlled assembly and dispersion of inorganic nanoparticles was achieved in earlier works by using molecular photo-switches as ligands, that were grafted through covalent bonds, including metal-thiolate or silane-silica bonds ${ }^{[13]}$. In these hybrid systems, two types of mechanisms mediate nanoparticular self-assembly: either the photoisomerization of the ligand modifies the electronic properties of the inorganic nanoparticles directly ${ }^{[14-16]}$, or the photo-isomerization of the ligand modifies the interaction between the nanoparticles and their surrounding environments, or between nanoparticles, which further mediates self-assembly or aggregation ${ }^{[17,18]}$. In particular, Klajn et al. demonstrated that when azobenzenes are used as ligands for spherical gold nanoparticles (5.6 nm in diameter), the gold nanoparticles that are initially dispersed in solution, aggregate under irradiation with UV light ${ }^{[17]}$. The origin of this aggregation was attributed to the azobenzene ligands switching into the cis-form, that has an increased dipole moment, where the dipole-dipole interaction upon the long-pair electrons of the $c i s-\mathrm{N}=\mathrm{N}$ bond makes the particles attractive to each other. 
This photo-controlled system has already been applied to control catalytic activity ${ }^{[19-20]}$, liquid crystal phase transition ${ }^{[21-22]}$, and photo-imaging and printing ${ }^{[23]}$. Reversely, it has also been shown that emissive nanoparticles can trigger the photo-switching of the ligands ${ }^{[24]}$.

Here, we extend this research towards investigating the light-triggered assembly of nanoplatelets, as two dimensional nano-objects. CdSe nanoparticles are well-known as quantum dots and are widely used in sensing, imaging and display systems and devices ${ }^{[25-31]}$ thanks to their size-dependent luminescence due to quantum confinement effects ${ }^{[32-34]}$. Beyond isotropic spherical particles, nanoplatelets of CdSe were developed recently ${ }^{[35]}$. The nanoplatelets contain faster carrier recombination than nanorods or quantum dots, and they are characterized by very sharp emission peak and low threshold lasing ${ }^{[36-38]}$. Upon controlling the vertical thickness through synthetic processes, the NPLs could demonstrate blue, green or yellow color emission, depending on whether three, four or five monolayers were formed, red emission could also be performed by CdS-CdSe-CdS core-shell hybrids ${ }^{[39,40]}$ [Figure 6.1a]. The emission of three primary colors allow the nanoplatelets to be a competitive candidate in lasing, imaging and optical display applications ${ }^{[41]}$.

Unlike spherical particles, which typically form lager aggregates randomly, twodimensional plate-like objects display a tendency to stack face-to-face into column-like supra structures to achieve lowest specific surface area and surface energy ${ }^{[42,43]}$. In particular, the CdSe nanoplatelets can self-assemble into columnar supra-particular ribbons by stacking face-to-face (Cd-001 site) from non-polar environment by anti-solvent addition ${ }^{[44]}$ or slow evaporation process ${ }^{[45]}[$ Figure 6.1b]. In the stacking ribbons, emission is strongly polarized in the direction perpendicular to the axis ${ }^{[44]}$, meanwhile the photoluminescence quantum 
yield and lifetime was decreased by increasing the ribbon length. Indeed, ultra-long exciton transfer among the ribbons leads to efficient homo-Förster resonance energy transfer and transport of the excitons increases the probability of its trapping by non-emissive nanoplatelets, which results in strong quenching of the photoluminescence. Hence the ultralong range exciton migration capability suggested potential application of the stacking nanoplatelets for light harvesting devices ${ }^{[46]}$.

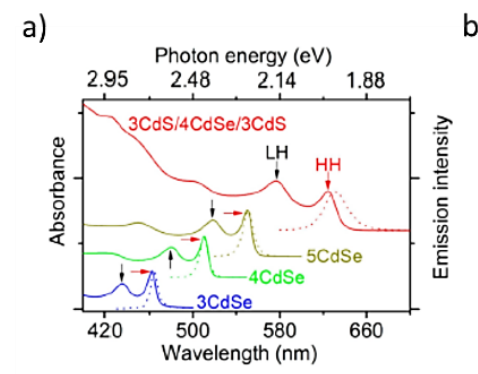

b) Dispersed single nano-platelets Self-aggregated ribbons
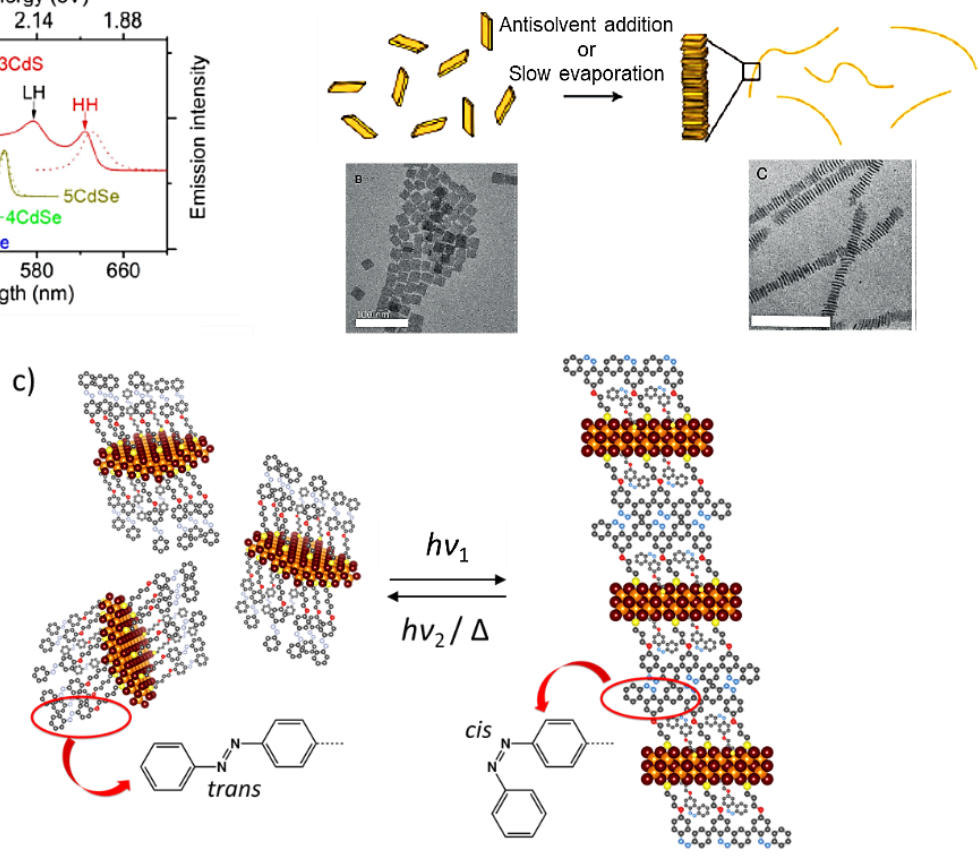

Figure 6.1. a) Absorption (solid lines) and emission (dashed lines) spectra of CdSe nanoplatelets emitting at $462 \mathrm{~nm}$ (3 monolayers), $512 \mathrm{~nm}$ (4 monolayers), $550 \mathrm{~nm}$ (5 monolayers) and $630 \mathrm{~nm}$ (CdSCdSe-CdS core-shell hybrids) in hexane solution. The black and red arrows indicate the electron/light hole ( $\mathrm{LH})$ and heavy hole $(\mathrm{HH})$ transitions respectively. Adapted with permission from Ref [39]. Copyright 2015 American Chemical Society. b) Self-aggregation of nanoplatelets into supra particular ribbons by anti-solvation or evaporation (scale bar $=100 \mathrm{~nm}$ (left) and $20 \mathrm{~nm}$ (right)). Adapted with permission from Ref [45]. Copyright 2016 Wiley-VCH. c) Photo-controlled switching of azobenzene ligands and self-assembly of the nanoplatelets. 
However, both the anti-solvation ${ }^{[44]}$ and the evaporation approach ${ }^{[45]}$ yield irreversible processes, and they require external chemical consumption. Here, we propose a new approach to form the ribbons of nanoplatelets. Azobenzene was chosen as photo control device to be conjugated to CdSe nanoplatelets. In the trans-state, with low ligand dipole moment the nanoplatelets are dispersed in a colloidal system. Once the azobenzene are switched to cis- form, the large dipole moment of cis-azobenzene drives the nanoplatelets together and form the stack. Thereby the generation of ribbons can be controlled under light as stimuli reversibly without any further chemical usage and waste [Figure 6.1c].

\subsection{Results and discussion}

Thiol ligands are widely used to stabilize colloidal inorganic semi-conductors and noble metal nanoparticles ${ }^{[17,47]}$. In agreement with hard and soft Lewis acid and bases Pearson's theory ${ }^{[48,49]}$, since the Cd-S interaction is favored with respect to the Cd-COO- interaction, ligand exchange of CdSe quantum dots from oleic acid to thiol ligand was found irreversible ${ }^{[50]}$. Therefore, the thiol group was chosen as terminal group of the ligand to attach to the nanoplatelets. In previous reports ${ }^{[44,45]}$, the determining factor of the distance between stacking nanoplatelets in the ribbon and its influence on the fluorescence luminescence performance remained an open question. Hence, we have introduced alkyl chain with different lengths as spacers between the azobenzene and the CdSe moieties, with the aim to control the inter-particular distance in the stacking ribbons so as to perform differed fluorescence emission. For ligands $\mathbf{4}-\mathbf{6}$, a tert-butyl $(t-\mathrm{Bu})$ group was introduced on the para- position of the azobenzene moiety to decrease possible $\pi-\pi$ stacking between adjacent 
nanoplatelets without deteriorating the electronic properties of the molecular switch. [Figure 6.2a, S6.1].

Considering the mono-methoxy azobenzene has a dipole moment $\sim 1 \mathrm{D}$ in the trans-form and $\sim 5 \mathrm{D}$ in the $c i s$ - form ${ }^{[51]}$, chloroform was chosen as solvent in this study, as it has a dipole moment $(\sim 1.01 \mathrm{D})$ that is similar to the trans-azobenzene. On the UV/Vis absorption spectrum of the ligand in chloroform solution, the trans-form $n-\pi *$ transition band was found at $\lambda_{\max }=350 \mathrm{~nm}$, the $\pi-\pi^{*}$ transition band was found at $440 \mathrm{~nm}$. After one minute of UV light $(\lambda=365 \mathrm{~nm})$ illumination, the trans-to-cis conversion takes place with decreased $\mathrm{n}-\pi^{*}$ transition band and increased $\pi-\pi^{*}$ transition band [Figure 6.2b, Figure S6.2].

a)

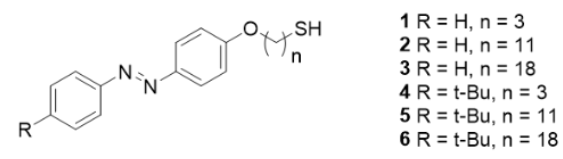

b)

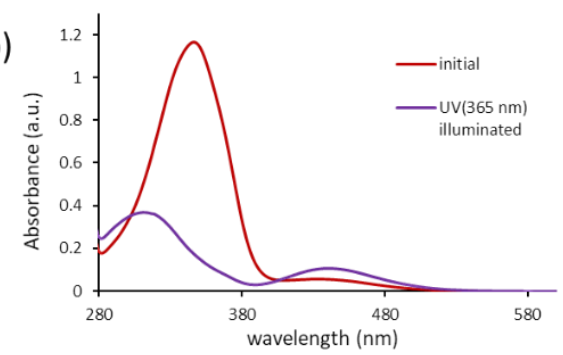

c)

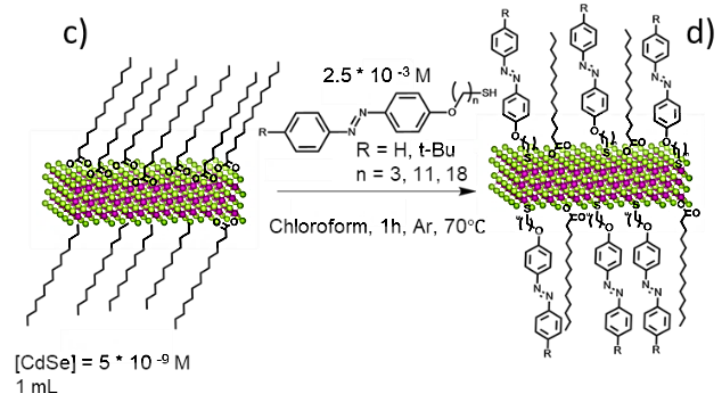

d)

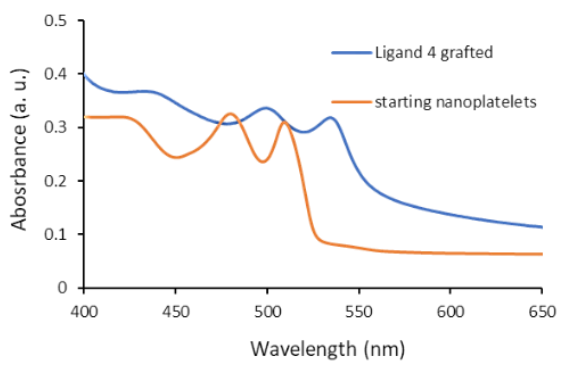

Figure 6.2. a) Azobenzene derivatives used as ligands in this study. b) UV/Vis absorption spectrum of ligand 1 in chloroform ( $\mathrm{c}=70 \mu \mathrm{M}$, UV illumination: $\lambda=365 \mathrm{~nm}, 1 \mathrm{~min})$. c) Procedure to functionalize the nanoplatelets with azobenzene derivatives. d) UV/Vis absorption spectrum of the CdSe nanoplatelets before and after functionalization with ligand 4. 
The CdSe nanoplatelets were freshly prepared by adapting known procedures with oleic acid as a stabilizing ligand ${ }^{[52]}$. When dispersed in chloroform, these platelets were characterized by two absorption bands at $\lambda_{\max }=509 \mathrm{~nm}$ and $\lambda_{\max }=480 \mathrm{~nm}$, indicating that the platelets have a vertical thickness of 4 monolayers $^{[37]}[$ Figure 6.1a, Figure 6.2d]. The two absorption bands correspond to the electron/heavy-hole transition and the electron/lighthole transition respectively, wherein the heavy-hole and light-hole are photon emission induced electron empty spaces with different energy levels ${ }^{[40]}$. The concentration was adjusted as $[\mathrm{CdSe}] \approx 5.08 \mathrm{nM}^{[53]}$, the lateral surface area was $\sim 420 \pm 100 \mathrm{~nm}^{2}$ on average [Table S6.1]. After a ligand exchange procedure aiming at replacing the oleic acid with azobenzene-thiol ligands, three precipitation/re-dispersion cycles were performed in order to remove the excess of azobenzene ligand and substituted oleic acid residues [Figure 6.2c]. A significant red shift was observed for both absorption bands; the electron/heavy-hole transition was found at $\lambda_{\max }=534 \mathrm{~nm}$, and electron/light-hole transition was found at $\lambda_{\max }=$ $500 \mathrm{~nm}$. This red shift is typical for thiol-capped metal chalcogenide nanoparticles ${ }^{[48-50]}$ and reflects a better delocalization of the exciton thanks to the strongest Cd-S bond with regards to $\mathrm{Cd}_{-\mathrm{COO}^{-}}$[Figure 6.2d]. No clear signal was detected by ${ }^{1} \mathrm{H}$ NMR measurements, which indicated that the substituted oleic acid ligands and excess of azobenzene ligands were removed by precipitation/re-dispersion procedure. We could thus exclude the influence of non-immobilized ligands from the colloidal solution. However, since the nanoplatelets were presented in very low concentration $\left(10^{-9} \mathrm{M}\right)$, the NMR characterization for ligand functionalization requires much more concentrated sample.

The self-assembly of the azobenzene-functionalized nanoplatelets was investigated by 
transmission electron microscopy (TEM) and dynamic light scattering (DLS), before and after illumination with UV light. The first studied sample is functionalized with ligand 1. On the TEM image, the nanoplatelets showed irregular lateral shapes. In the initial form, most of the nanoplatelets are randomly dispersed, with their lateral face lying on the TEM sample substrate. However, short face-to-face stacks up to ten nanoplatelets were found with their plain perpendicular to the substrate. The periodicity of the short stacks was $4.6 \pm 0.5 \mathrm{~nm}$, since a 4 monolayer nanoplatelet has a thickness of $1.2 \mathrm{~nm}^{[37]}$, the distance between two single nanoplatelets is $3.4 \mathrm{~nm}$ [Figure 6.3a]. We assumed that these short stacks may be induced by inter-particular $\pi-\pi$ stacking of the trans-azobenzene ligands. On the TEM image of UV illuminated sample, most of the nanoplatelets are assembled face-to-face, forming supra-particular ribbons with significant longer axis than the trans- state local aggregates [Figure 6.3b]. The longest supra-particular ribbon was found up to $500 \mathrm{~nm}$ long and comprised more than 100 nanoplatelets. The long ribbons showed same periodicity as the short stacks that were observed in the trans-form.

a)

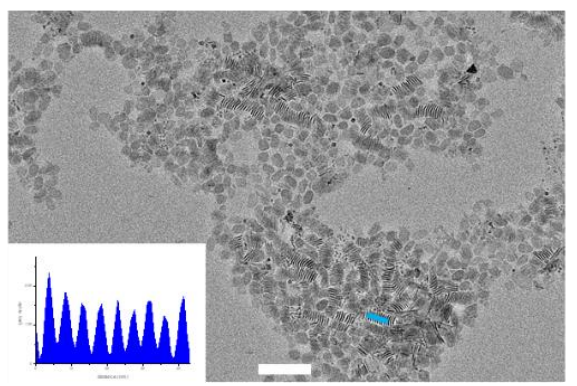

b)

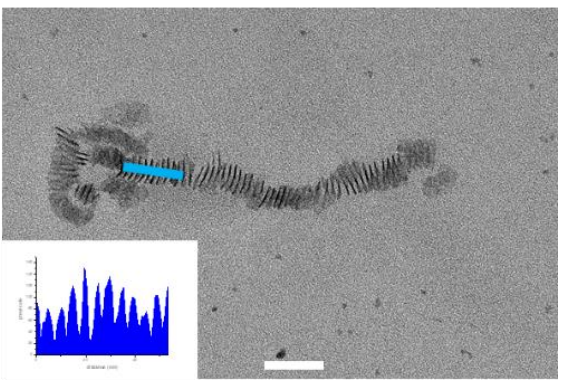

Figure 6.3. TEM image of ligand 1 functionalized nanoplatelets. a) In the initial state (scale bar $=100$ $\mathrm{nm}$ ). b) After UV illumination (scale bar $=50 \mathrm{~nm}$ ). Insets: stacking profile perpendicular to the ribbon axis according to image grey scale.

The TEM investigations thus demonstrate that photo-induced stacking of the 
nanoplatelets occurs. However, since short stacks have been observed also before UV irradiation, we set out to remove this unwanted aggregation in the trans-form of the azobenzene. We therefore studied this sample in chloroform solution by DLS measurements at $20^{\circ} \mathrm{C}$ and compared the results to the same experiments for particles functionalized by ligand 4, which was modified with para-position $t$-Bu group in order to increase the ligand bulkiness and disturb the trans- state $\pi-\pi$ stacking $^{[54]}$ [Table 6.1].

Table 6.1. Dynamic light scattering (DLS) analysis of nano-platelets covered with either ligand 1, or ligand 4.

Ligand 1

\begin{tabular}{ccccc}
\hline step & Size $(\mathrm{nm})$ & Sigma $(\mathrm{nm})$ & Size $(\mathrm{nm})$ & Sigma $(\mathrm{nm})$ \\
$\begin{array}{c}\text { initial } \\
\lambda=365 \mathrm{~nm}\end{array}$ & 110 & 13 & 26 & 4 \\
$\begin{array}{c}1 \mathrm{~min} \\
\text { ambient light } \\
5 \text { min }\end{array}$ & 545 & 53 & 425 & 150 \\
$\begin{array}{c}\lambda=365 \mathrm{~nm} \\
1 \mathrm{~min}\end{array}$ & 616 & 26 & 58 & 20 \\
\hline
\end{tabular}

From DLS analysis, it appears that in the initial state, ligand 4 allows for a smaller hydrodynamic average size of the nanoplatelets than ligand $\mathbf{1}$. Thus, we conclude that a bulky group such as $t$-Bu allows for a better initial dispersity of the nanoplatelets. In addition, UVillumination $(\lambda=365 \mathrm{~nm}, 1 \mathrm{~min})$ causes an increase of the hydrodynamic size of the nanoplatelets, which indicates photo-induced stacking of the platelets in solution. At last, the stacking process is also reversible since relaxation of the system under ambient light allows for recovery of the initial size. 
The influence of the $t$-Bu group on the supra-particular assembling properties was investigated by TEM also. For the sample with $\mathbf{4}$ used as a ligand, only individual dispersed platelets were found in the initial state in TEM image, without local aggregations [Figure 6.4a]. After UV irradiation, long supra-particular ribbons were found with lengths reaching up to $1 \mu \mathrm{m}$, and contained more than 200 individual nanoplatelets [Figure 6.4b]. The periodicities in sample with ligand 1 [Figure 6.3a and 6.3b] and ligand 4 [Figure 6.4b] were found the same as $4.6 \pm 0.5 \mathrm{~nm}$.

a)

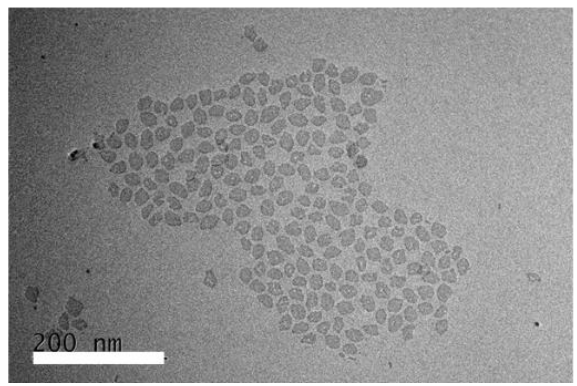

b)

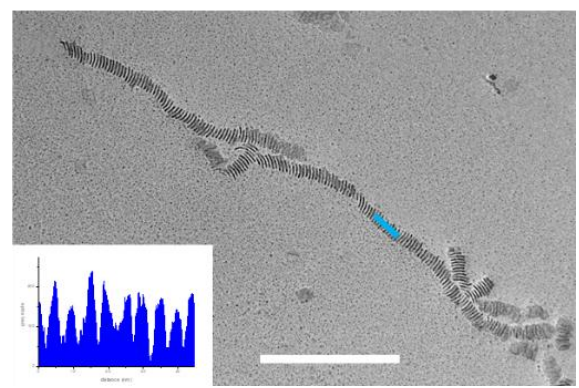

Figure 6.4. TEM image of nanoplatelets functionalized with ligand 4. a) In the initial state. b) After UV illumination, inset: stacking profile perpendicular to the ribbon axis according to image grey scale (all scale bars $=200 \mathrm{~nm}$ ).

Ligand 6 was also employed to study the influence of the ligand spacer length on the selfassembly. On the TEM image of the UV illuminated nanoplatelets, self-assembled long ribbons up to $\mu \mathrm{m}$ scale length was also found with the same periodicity as ligand $\mathbf{1}$ or ligand 4 [Figure 6.5a]. Interestingly, in a more condensed area, the nanoplatelets with cis-1 and cis4 yielded bundled patterns, where the individual ribbons were aligned close to each other and oriented in a similar direction [Figure 6.5b, Figure 6.5c]. In the sample with cis-6, the ribbons did not form bundles but crossed each other in networks [Figure 6.5d]. However, a 
thorough statistical analysis will have to be carried out in order to conclude whether this difference in morphology could be significant. If so, considering that the fluorescence emission is strongly polarized perpendicular to the ribbon axis, we anticipate that the difference between bundled and crossed networks could yield an interesting behaviour in optical display devices.

a)

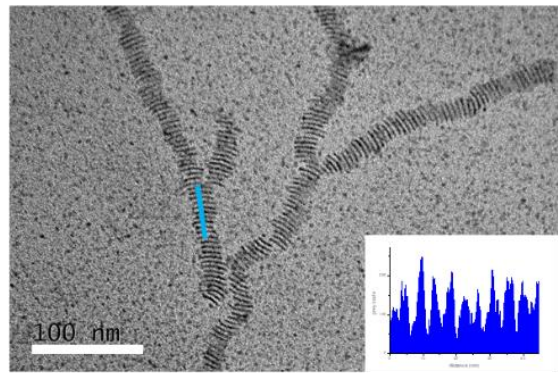

c)

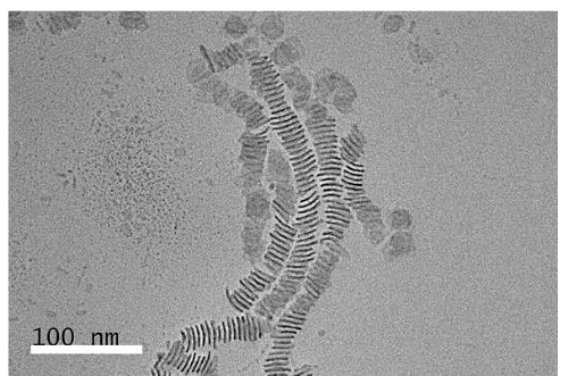

b)

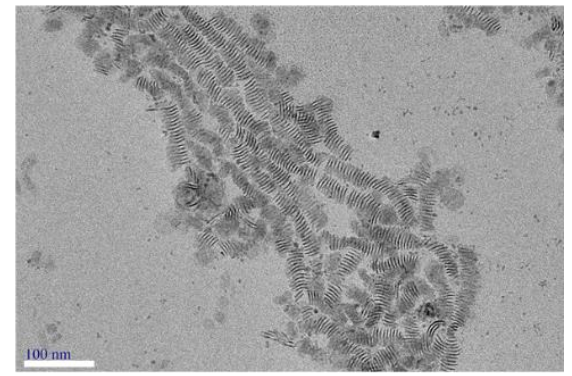

d)

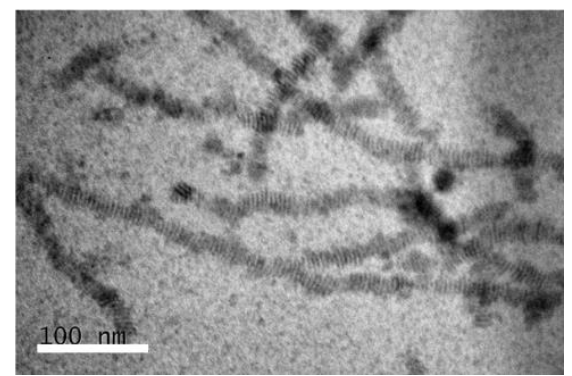

Figure 6.5. a) TEM image of nanoplatelets with ligand $\mathbf{6}$ after UV illumination, inset: stacking profile perpendicular to the ribbon axis according to image grey scale. b) TEM image of bundled supraparticular alignment with ligand $\mathbf{1}$ after UV illumination. c) TEM image of bundled supra-particular alignment with ligand $\mathbf{4}$ after UV illumination. d) TEM image of crossing supra-particular alignments with ligand 6 after UV illumination (all scale bars $=100 \mathrm{~nm}$ ).

In earlier reports, supra-particular ribbons of nanoplatelets were obtained by antisolvent $\mathrm{MeOH}$ precipitation or evaporation process from hexane solution ${ }^{[44,45]}$. Here in contrast, we use chloroform as a good solvent for azobenzenes in both their trans- and cis- form, hence 
we exclude the influence of solvophobic effects.

In this study, ligands with different various of molecular length were involved, where initial ligand oleic acid has a length for $19.9 \AA$, ligand 1 for $15.0 \AA$ in trans- form and 11.2 $\AA$ in $c i s$ - from terminal $\mathrm{S}$ atom to the $\mathrm{N}$ atom with long pair electrons, cis- ligand $\mathbf{4}$ for 11.2 $\AA$ and cis- ligand 6 for $29.8 \AA$ [Figure 6.6]. However, in the experiments with ligand 1, 4, and 6, all the stacks that were formed, with or without dipole-dipole interaction and with different spacer lengths showed the same inter-particle periodicity $(4.6 \pm 0.5 \mathrm{~nm})$, i.e. in all cases the average distance between two platelets was the same $(3.4 \pm 0.5 \mathrm{~nm})$, independently from the length of the ligand.

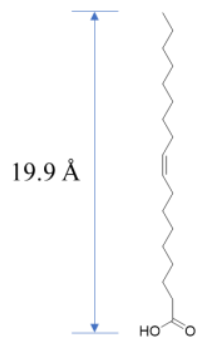

oleic acid

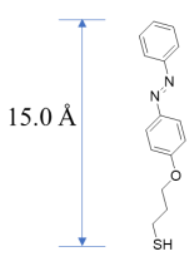

trans- 1
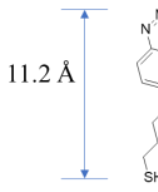

cis- 1
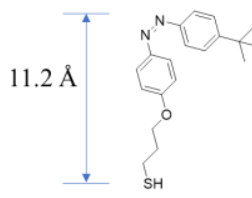

$29.8 \AA$

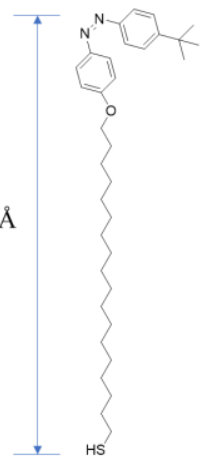

cis- 4

cis- 6

Figure 6.6. Molecular lengths of ligands discussed in this chapter.

In earlier reports with oleic acid as ligand ${ }^{[44-46]}$, the stacking periodicity was $\sim 5.1 \mathrm{~nm}$ and electron/heavy-hole absorption peak was found at $510 \mathrm{~nm}$. In this study, the nanoplatelets were functionalized with thiol terminal ligands, which causes red shift of the absorption peak to $534 \mathrm{~nm}$, i.e. decreased electron energy of the CdSe moiety, meanwhile the periodicity was $\sim 4.6 \mathrm{~nm}$. The relationship between absorption peak and periodicity shows that in these works, 
the periodicity is only marginally influenced by the ligand dipole-dipole interaction, although this interaction makes the nanoplatelets more attractive to each other and induces stacking. One possible reason could be that not all the oleic acide is removed during ligand exchange, hence the oleic acid determines the platelet-to-platelet distance. Current investigations aim at better etching the oleic acid during ligand exchange.

\subsection{Conclusions}

We synthesized a series of azobenzene switches with a thiol function that allows using them as ligands for inorganic nanoplatelets. In chloroform, since the trans-azobenzene has a similar dipole moment to the environment, the nanoplatelets were mostly well-dispersed. However, short stacks were also observed on TEM images with trans-azobenzene, which we assumed to ligand $\pi-\pi$ stacking induced local aggregation. Upon illumination, we have shown that the switching of the ligands promotes the self-assembly of the nanoplatelets into supraparticular ribbons. Further, the azobenzene ligands were modified with $t$-Butyl group on the para-position to induce steric hindrance and thus disturb the trans- state $\pi$ - $\pi$ stacking, so local aggregates cannot be found anymore, and supra-particular assembly is controlled by light entirely.

In follow-up work, we will focus on controlling the stacking periodicity, as well as characterizing the photo-emission performance of the ribbons by fluorescence spectral analysis, fluorescence microcopy and scanning electron microscopy-cathodoluminescence. 


\subsection{Acknowledgments}

Yesica Flores and Dr. Benoit Fleury are gratefully acknowledged for collaboration on this research program, that is also supported by a Van Gogh Fellowship (NUFFIC). The synthesis of the CdSe nanoplatelets, ligand functionalization, DLS analysis and part of the TEM measurements were performed by Yesica Flores and Dr. Benoit Fleury in Université Paris Sorbonne. Dr. Rico Keim is gratefully acknowledged for help with the TEM measurements that were performed at the University of Twente.

\subsection{Supporting information}

\subsubsection{Materials and methods}

All chemicals and solvents were purchased from commercial suppliers and used without further purification. ${ }^{1} \mathrm{H}$ NMR and ${ }^{13} \mathrm{C}$ NMR spectra were recorded in $\mathrm{CDCl}_{3}$ in a Bruker 400MHz Avance III NMR spectrometer. Chemical shifts ( $\delta$ ) are given in ppm with the residual solvent peak as the internal reference. The coupling constants $(J)$ are reported in hertz (Hz). NMR splitting patterns are designed as follows: $s$, singlet; $d$, doublet; $t$, triplet; $q$, quadruplet and m, multiplet. Analytical chromatography was performed on silica gel (60-200 mesh). High resolution mass spectra were registered using Waters/Micromass LCT Mass spectrometer with commercial compound Reserpine as internal standard. TEM measurements were performed using Philips CM300 microscope operating at $300 \mathrm{kV}$. TEM images were analyzed by software ImageJ. 


\subsubsection{Synthesis of the azobenzene ligands}

The synthesis of azobenzene ligands was performed by following known procedures ${ }^{[55]}$ [Figure S6.1].
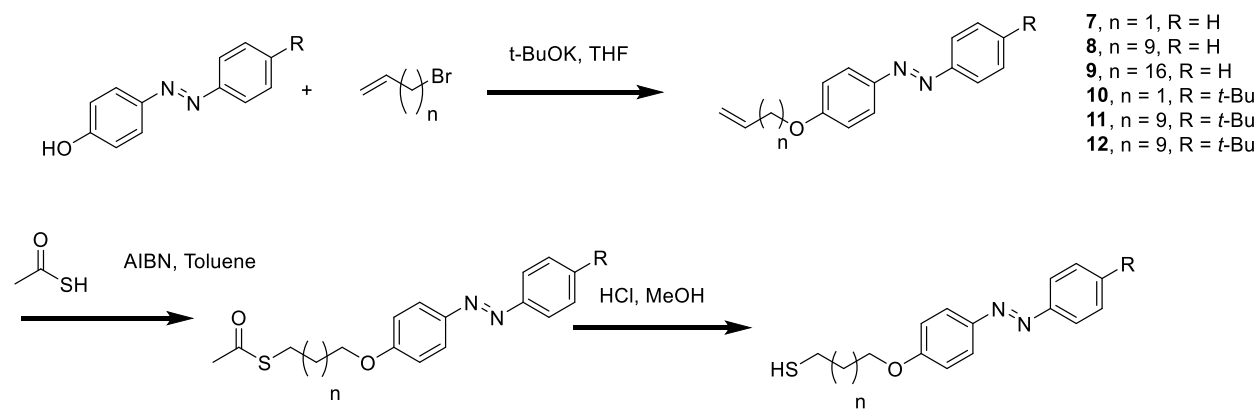

13, $\mathrm{n}=1, \mathrm{R}=\mathrm{H}$
14, $\mathrm{n}=9, \mathrm{R}=\mathrm{H}$
$15, \mathrm{n}=16, \mathrm{R}=\mathrm{H}$
$16, \mathrm{n}=1, \mathrm{R}=t-\mathrm{Bu}$
$17, \mathrm{n}=9, \mathrm{R}=t-\mathrm{Bu}$
$18, \mathrm{n}=9, \mathrm{R}=t-\mathrm{Bu}$

$1, n=1, R=H$

$2, \mathrm{n}=9, \mathrm{R}=\mathrm{H}$

$3, \mathrm{n}=16, \mathrm{R}=\mathrm{H}$

4, $\mathrm{n}=1, \mathrm{R}=t-\mathrm{Bu}$

$5, \mathrm{n}=9, \mathrm{R}=t-\mathrm{Bu}$

6, $\mathrm{n}=9, \mathrm{R}=t-\mathrm{Bu}$

Figure 6.5. Synthesis route of the ligands.

4-(allyloxy)phenyl-2-phenyldiazene (7)<smiles>C=CCOc1ccc(/N=N/c2ccccc2)cc1</smiles>

In a flame-dried three neck round flask, 4-(phenyldiazenyl)phenol (0.793 g, 4 mmol, 1 eq.), 3-bromoprop-1-ene (0.484 g, $4 \mathrm{mmol}, 1$ eq.) and potassium $t$-butoxide ( $0.583 \mathrm{~g}, 5.2 \mathrm{mmol}$, 1.3 eq.) were dissolved in $11 \mathrm{ml}$ of dry THF. This mixture was stirred and refluxed overnight under $\mathrm{N}_{2}$, reaction progress was monitored by TLC. Then the reaction mixture was cooled down to room temperature, the solvent was evaporated. The residues were dissolved in 50 $\mathrm{ml}$ of dichloromethane and washed with water $(30 \mathrm{ml} \times 3)$. The organic phase was collected and dried over $\mathrm{MgSO}_{4}$. The dichloromethane was evaporated and the residues were purified 
by column chromatography on silica gel (ethyl acetate : hexane $=1: 20$ ). The product was collected as orange solid, $0.709 \mathrm{~g}$, yield $74 \%$, and the ${ }^{1} \mathrm{H}$ NMR spectrum was in accordance with previous reports ${ }^{[56]}$.

${ }^{1} \mathrm{H}$ NMR $\left(400 \mathrm{MHz}, \mathrm{CDCl}_{3}\right) \delta=4.62\left(\mathrm{~d}, J=5.3 \mathrm{~Hz}, 2 \mathrm{H}, \mathrm{O}-\mathrm{CH}_{2}\right), 5.39\left(\mathrm{~m} .1 \mathrm{H},=\mathrm{CH}_{2} \mathrm{Z}-\right.$ position), $5.52\left(\mathrm{dd}, J=17.3 \mathrm{~Hz}, 1.6 \mathrm{~Hz}, 1 \mathrm{H},=\mathrm{CH}_{2}\right.$ E-position), 6.13 (ddt, $J=17.3 \mathrm{~Hz}, 10.5$ $\mathrm{Hz}, 5.2 \mathrm{~Hz}, 1 \mathrm{H},-\mathrm{CH}=) .7 .10(\mathrm{~d}, J=9.0 \mathrm{~Hz}, 2 \mathrm{H}, \mathrm{Ar}-\mathrm{H}), 7.52$ (m, 1H, Ar-H), 7.59 (m, 2H, ArH), 8.04 ppm (m, 4H, Ar-H).

\section{3-(4-(phenyldiazenyl)phenoxy)propyl-ethanethioate (13)}<smiles>CC(=O)SCCCOc1ccc(N=Nc2ccccc2)cc1</smiles>

In a flame dried three neck round bottom flask, compound 7 (0.476 g, 2 mmol, 1 eq.) and azobisisobutyronitrile (AIBN) (0.690 g, $4.2 \mathrm{mmol}, 2.1$ eq.) were dissolved in $10 \mathrm{ml}$ of dry toluene. Thioacetic acid $(0.320 \mathrm{~g}, 4.2 \mathrm{mmol}, 2.1 \mathrm{eq})$ was added to the mixture dropwise under stirring. After addition the mixture was refluxed for 6 hours. The reaction progress was monitored by TLC. Then the flask was cooled down to room temperature. The solution was diluted by $30 \mathrm{ml}$ of toluene, washed with saturated $\mathrm{NaHCO}_{3}$ solution in water $(30 \mathrm{ml})$ and water $(30 \mathrm{ml} \times 2)$. The organic phase was collected and dried over $\mathrm{MgSO}_{4}$. The solvent was evaporated, the residues were purified by column chromatography on silica gel (ethyl acetate : hexane $=1: 10$ ). The product was collected as orange solid, $0.557 \mathrm{~g}$, yield $88 \%$.

${ }^{1} \mathrm{H} \mathrm{NMR}\left(400 \mathrm{MHz}, \mathrm{CDCl}_{3}\right) \delta=2.10\left(\mathrm{~m}, 2 \mathrm{H}, \mathrm{C}-\mathrm{CH}_{2}-\mathrm{C}\right), 2.35\left(\mathrm{~s}, 3 \mathrm{H},-\mathrm{COCH}_{3}\right), 3.08(\mathrm{t}, J=$ 
$\left.7.1 \mathrm{~Hz}, 2 \mathrm{H}, \mathrm{C}-\mathrm{CH}_{2}-\mathrm{S}\right), 4.03\left(\mathrm{t}, J=6.1 \mathrm{~Hz}, 2 \mathrm{H}, \mathrm{O}-\mathrm{CH}_{2}\right), 7.00(\mathrm{~d}, J=9.0 \mathrm{~Hz}, 2 \mathrm{H}, \mathrm{Ar}-\mathrm{H}), 7.46$ (m, 1H, Ar-H), 7.53 (dd, J=8.3 Hz, 6.6 Hz, 2H, Ar-H), 7.96 ppm (m, 4H, Ar-H).

${ }^{13} \mathrm{C} \mathrm{NMR}\left(100 \mathrm{MHz}, \mathrm{CDCl}_{3}\right) \delta=25.55,28.96,30.41,66.24,114.46,122.32,124.59,128.78$, $130.15,146.69,152.36,161.06,195.38 \mathrm{ppm}$.

TOF MS ES+ $\left(\mathrm{M}+\mathrm{H}^{+}\right)$Calculated for $\left[\mathrm{C}_{17} \mathrm{H}_{19} \mathrm{~N}_{2} \mathrm{O}_{2} \mathrm{~S}^{+}\right]$: 315.1162, found 315.2200.

\section{3-(4-(phenyldiazenyl)phenoxy)propane-1-thiol (1)}<smiles>SCCCOc1ccc(/N=N/c2ccccc2)cc1</smiles>

Compound 13 ( $0.157 \mathrm{~g}, 0.5 \mathrm{mmol}, 1$ eq.) was placed in a flame dried three neck round flask. Then $12 \mathrm{ml}$ of dry $\mathrm{MeOH}$ was added, followed by $1.4 \mathrm{ml}$ of $\mathrm{HCl}$ in $\mathrm{MeOH}$ solution (1 M). The mixture was refluxed for $90 \mathrm{~min}$. Then the solution was cooled down to room temperature. Excess $\mathrm{HCl}$ was neutralized with $\mathrm{NaOH}$ in water solution $(1 \mathrm{M})$. The mixture was dissolved in $30 \mathrm{ml}$ of dichloromethane and washed with water $(30 \mathrm{ml} \times 2)$. The organic phase was collected and dried over $\mathrm{MgSO}_{4}$. The dichloromethane was evaporated and the residues were purified by column chromatography on silica gel (ethyl acetate : hexane $=1$ : 15), product was collected as orange solid, $0.059 \mathrm{~g}$, yield 44\%, it's ${ }^{1} \mathrm{H}$ NMR spectrum was in accordance with previous report ${ }^{[57]}$.

${ }^{1} \mathrm{H}$ NMR $\left(400 \mathrm{MHz}, \mathrm{CDCl}_{3}\right) \delta=1.42(\mathrm{t}, J=8.1 \mathrm{~Hz}, 1 \mathrm{H},-\mathrm{SH}), 2.13\left(\mathrm{~m}, 2 \mathrm{H}, \mathrm{C}-\mathrm{CH}_{2}-\mathrm{C}\right), 2.77$ (dt, $\left.J=8.2,6.9 \mathrm{~Hz}, 2 \mathrm{H}, \mathrm{S}-\mathrm{CH}_{2}\right), 4.17\left(\mathrm{t}, J=5.9 \mathrm{~Hz}, 2 \mathrm{H}, \mathrm{O}-\mathrm{CH}_{2}\right), 7.01(\mathrm{~d}, J=9.0 \mathrm{~Hz}, 2 \mathrm{H}$, Ar-H), 7.45 (m, 1H, Ar-H), 7.51 (m, 2H, Ar-H), 7.90 ppm (m, 4H, Ar-H). 


\section{1-phenyl-2-(4-(undec-10-en-1-yloxy)phenyl)diazene (8)}

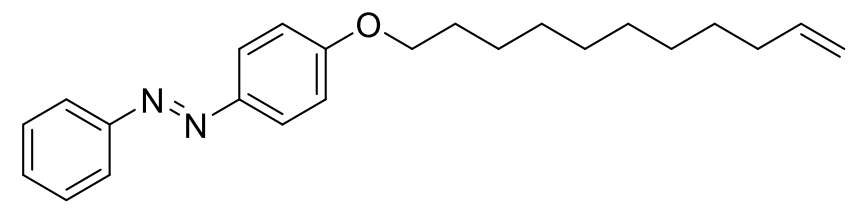

In a flame-dried three neck round flask, 4-(phenyldiazenyl)phenol (0.500 g, $2.52 \mathrm{mmol}, 1$ eq.), 11-bromoundec-1-ene (0.588 g, $2.52 \mathrm{mmol}, 1$ eq.) and potassium $t$-butoxide ( $0.367 \mathrm{~g}$, $3.28 \mathrm{mmol}, 1.3$ eq.) were dissolved in $7.5 \mathrm{ml}$ of dry THF. This mixture was stirred and refluxed overnight under $\mathrm{N}_{2}$, reaction progress was monitored by TLC. Then the reaction mixture was cooled down to room temperature, the solvent was evaporated. The residues were dissolved in $50 \mathrm{ml}$ of dichloromethane and washed with water $(30 \mathrm{ml} \times 3)$. The organic phase was collected and dried over $\mathrm{MgSO}_{4}$. The dichloromethane was evaporated and the residues were purified by column chromatography on silica gel (ethyl acetate : hexane $=1$ : 50). The product was collected as orange solid, $0.550 \mathrm{~g}$, yield $62 \%$, its ${ }^{1} \mathrm{H}$ NMR spectrum was in accordance with previous report ${ }^{[58]}$.

${ }^{1} \mathrm{H}$ NMR $\left(400 \mathrm{MHz}, \mathrm{CDCl}_{3}\right) \delta=1.34\left(\mathrm{~m}, 10 \mathrm{H}, \mathrm{C}-\left(\mathrm{CH}_{2}\right)_{5}-\mathrm{C}\right), 1.47$ (m, 2H, O-C-C-CH$), 1.81$ $\left(\mathrm{m}, 2 \mathrm{H}, \mathrm{O}-\mathrm{C}-\mathrm{CH}_{2}\right), 2.04\left(\mathrm{~m}, 2 \mathrm{H}, \mathrm{C}=\mathrm{C}-\mathrm{CH}_{2}\right), 4.04\left(\mathrm{t}, J=6.6 \mathrm{~Hz}, 2 \mathrm{H}, \mathrm{O}-\mathrm{CH}_{2}\right), 4.94(\mathrm{~m}, 1 \mathrm{H}$, $\mathrm{C}=\mathrm{CH}_{2} \mathrm{Z}$ position), 5.00 (dd, $J=17.1,2.0 \mathrm{~Hz}, 1 \mathrm{H}, \mathrm{C}=\mathrm{CH}_{2} \mathrm{E}$ position), 5.82 (ddt, $J=16.9$, 10.2, 6.7 Hz, 1H, C-CH=C), 7.00 (d, J=9.0 Hz, 2H, Ar-H), 7.44 (m, 1H, Ar-H), 7.50 (m, 2H, Ar-H), 7.89 ppm (m, 4H, Ar-H).

\section{1-(4-(phenyldiazenyl)phenoxy)undecyl-ethanethioate (14)}




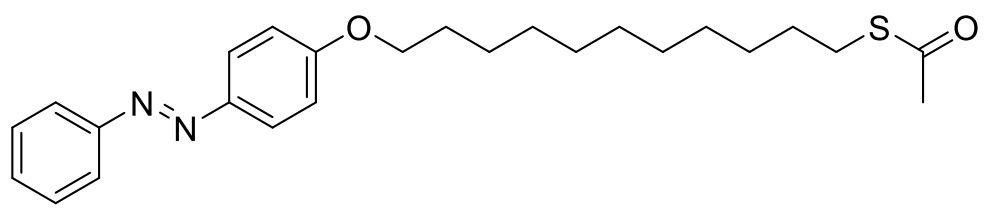

In a flame dried three neck round flask, compound 8 (0.175 g, 0.5mmol, 1eq.) and azobisisobutyronitrile (AIBN) (0.172 g, $1.05 \mathrm{mmol}, 2.1 \mathrm{eq}$.$) were dissolved in 5 \mathrm{ml}$ of dry toluene. Thioacetic acid $(0.080 \mathrm{~g}, 1.05 \mathrm{mmol}, 2.1 \mathrm{eq}$.$) was added to the mixture dropwise$ under stirring. After addition the mixture was refluxed for 6 hours. The reaction progress was monitored by TLC. Then the flask was cooled down to room temperature. The solution was diluted by $30 \mathrm{ml}$ of toluene, washed with saturated $\mathrm{NaHCO}_{3}$ solution in water $(30 \mathrm{ml})$ and water $(30 \mathrm{ml} \times 2)$. The organic phase was collected and dried over $\mathrm{MgSO}_{4}$. The solvent was evaporated, the residues were purified by column chromatography on silica gel (ethyl acetate : hexane $=1: 20$ ). The product was collected as orange solid, $0.222 \mathrm{~g}$, yield $92 \%$, its ${ }^{1} \mathrm{H}$ NMR spectrum was in accordance with previous reports ${ }^{[22]}$.

${ }^{1} \mathrm{H}$ NMR $\left(400 \mathrm{MHz}, \mathrm{CDCl}_{3}\right) \delta=1.30\left(\mathrm{~m}, 14 \mathrm{H}, \mathrm{C}-\left(\mathrm{CH}_{2}\right)_{7}-\mathrm{C}\right), 1.56\left(\mathrm{~m}, 2 \mathrm{H}, \mathrm{S}-\mathrm{C}-\mathrm{CH}_{2}\right), 1.82$ (dt, $\left.J=14.6,6.7 \mathrm{~Hz}, 2 \mathrm{H}, \mathrm{O}-\mathrm{C}-\mathrm{CH}_{2}\right), 2.32\left(\mathrm{~s}, 3 \mathrm{H},-\mathrm{COCH}_{3}\right), 2.87$ (t, $\left.J=7.3 \mathrm{~Hz}, 2 \mathrm{H}, \mathrm{S}-\mathrm{CH}_{2}\right)$, $4.03\left(\mathrm{t}, J=6.6 \mathrm{~Hz}, 2 \mathrm{H}, \mathrm{O}-\mathrm{CH}_{2}\right), 7.00$ (d, $\left.J=9.0 \mathrm{~Hz}, 2 \mathrm{H}, \mathrm{Ar}-\mathrm{H}\right), 7.44$ (m, 1H, Ar-H), 7.50 (m, 2H, Ar-H), 7.89 ppm (m, 4H, Ar-H).

11-(4-(phenyldiazenyl)phenoxy)undecane-1-thiol (2)<smiles>SCCCCCCCCCCCOc1ccc(N=Nc2ccccc2)cc1</smiles> 
Compound 14 (0.214 g, $0.5 \mathrm{mmol}, 1$ eq.) was placed in a flame dried three neck round flask. Then $12 \mathrm{ml}$ of dry $\mathrm{MeOH}$ was added, followed by $1.4 \mathrm{ml}$ of $\mathrm{HCl}$ in $\mathrm{MeOH}$ solution $(1 \mathrm{M})$. The mixture was refluxed for 3 hours. Then the solution was cooled down to room temperature. Excess of $\mathrm{HCl}$ was neutralized with $\mathrm{NaOH}$ in water solution (1 M). The mixture was dissolved in $30 \mathrm{ml}$ of dichloromethane and washed with water $(30 \mathrm{ml} \times 2)$. The organic phase was collected and dried over $\mathrm{MgSO}_{4}$. Product was isolated by silica preparative TLC plate (ethyl acetate : hexane $=1: 40$ ) as orange solid, $0.069 \mathrm{~g}$, yield $36 \%$, its ${ }^{1} \mathrm{H}$ NMR spectrum was in accordance with previous report ${ }^{[59]}$.

${ }^{1} \mathrm{H}$ NMR $\left(400 \mathrm{MHz}, \mathrm{CDCl}_{3}\right) \delta=1.30\left(\mathrm{~m}, 10 \mathrm{H}, \mathrm{C}-\left(\mathrm{CH}_{2}\right)_{5}-\mathrm{C}\right), 1.33(\mathrm{t}, J=7.8 \mathrm{~Hz}, 1 \mathrm{H},-\mathrm{SH})$, $1.47\left(\mathrm{~m}, 2 \mathrm{H}, \mathrm{O}-\mathrm{C}-\mathrm{C}-\mathrm{CH}_{2}\right), 1.61\left(\mathrm{~m}, 2 \mathrm{H}, \mathrm{S}-\mathrm{C}-\mathrm{CH}_{2}\right), 1.82\left(\mathrm{dd}, J=8.3,6.4 \mathrm{~Hz}, 2 \mathrm{H}, \mathrm{O}-\mathrm{C}-\mathrm{CH}_{2}\right)$, 2.52 (q, $\left.J=7.4 \mathrm{~Hz}, 2 \mathrm{H}, \mathrm{S}-\mathrm{CH}_{2}\right), 4.04$ (t, $\left.J=6.5 \mathrm{~Hz}, 2 \mathrm{H}, \mathrm{O}-\mathrm{CH}_{2}\right), 7.00$ (d, $J=8.9 \mathrm{~Hz}, 2 \mathrm{H}, \mathrm{Ar}-$ H), 7.44 (m, 1H, Ar-H), 7.50 (m, 2H, Ar-H), 7.90 ppm (m, 4H, Ar-H).

\section{1-(4-(octadec-17-en-1-yloxy)phenyl)-2-phenyldiazene (9)}<smiles>C=CCCCCCCCCCCCCOc1ccc(N=Nc2ccccc2)cc1</smiles>

In a flame-dried three neck round flask, 4-(phenyldiazenyl)phenol (0.396 g, 2 mmol, 1 eq.), 18-bromooctadec-1-ene ( $0.663 \mathrm{~g}, 2 \mathrm{mmol}, 1$ eq. $)$ and potassium $t$-butoxide $(0.291 \mathrm{~g}, 2.6$ mmol, 1.3 eq.) were dissolved in $7.5 \mathrm{ml}$ of dry THF. This mixture was stirred and refluxed overnight under $\mathrm{N}_{2}$, reaction progress was monitored by TLC. Then the reaction mixture was cooled down to room temperature, the solvent was evaporated. The residues were dissolved 
in $50 \mathrm{ml}$ of dichloromethane and washed with water $(30 \mathrm{ml} \times 3)$. The organic phase was collected and dried over $\mathrm{MgSO}_{4}$. The dichloromethane was evaporated and the residues were purified by column chromatography on silica gel (ethyl acetate $:$ hexane $=1: 50)$. The product was collected as orange solid, $0.475 \mathrm{~g}$, yield 53\%

${ }^{1} \mathrm{H}$ NMR $\left(400 \mathrm{MHz}, \mathrm{CDCl}_{3}\right) \delta=1.30\left(\mathrm{~m}, 26 \mathrm{H}, \mathrm{C}-\left(\mathrm{CH}_{2}\right)_{13}-\mathrm{C}\right), 1.83\left(\mathrm{~m}, 2 \mathrm{H}, \mathrm{O}-\mathrm{C}-\mathrm{CH}_{2}\right), 2.05$ $\left(\mathrm{m}, 2 \mathrm{H}, \mathrm{C}=\mathrm{C}-\mathrm{CH}_{2}\right), 4.04\left(\mathrm{t}, J=6.6 \mathrm{~Hz}, 2 \mathrm{H}, \mathrm{O}-\mathrm{CH}_{2}\right), 4.97\left(\mathrm{~m}, 2 \mathrm{H}, \mathrm{C}=\mathrm{CH}_{2}\right), 5.81(\mathrm{~m}, 1 \mathrm{H}, \mathrm{C}-$ $\mathrm{CH}=\mathrm{C}), 7.01(\mathrm{~m}, 2 \mathrm{H}, \mathrm{Ar}-\mathrm{H}), 7.44(\mathrm{~m}, 1 \mathrm{H}, \mathrm{Ar}-\mathrm{H}), 7.48(\mathrm{~m}, 2 \mathrm{H}, \mathrm{Ar}-\mathrm{H}), 7.91 \mathrm{ppm}(\mathrm{m}, 4 \mathrm{H}, \mathrm{Ar}-$ H).

${ }^{13} \mathrm{C}$ NMR $\left(100 \mathrm{MHz}, \mathrm{CDCl}_{3}\right) \delta=14.28,22.82,25.43,26.18,27.07,29.12,29.33,29.69$, $29.74,29.77,29.80,29.84,29.85,31.76,33.99,34.82,68.50,114.22,114.81,122.68$, $124.89,129.14,130.41,139.40,146.98,152.93,161.85$ ppm.

TOF MS ES+ $(\mathrm{M}+\mathrm{H})$ Calculated for $\left[\mathrm{C}_{30} \mathrm{H}_{45} \mathrm{~N}_{2} \mathrm{O}^{+}\right]$: 449.3526, found 449.3500 .

\section{8-(4-(phenyldiazenyl)phenoxy)octadecyl-ethanethioate (15)}

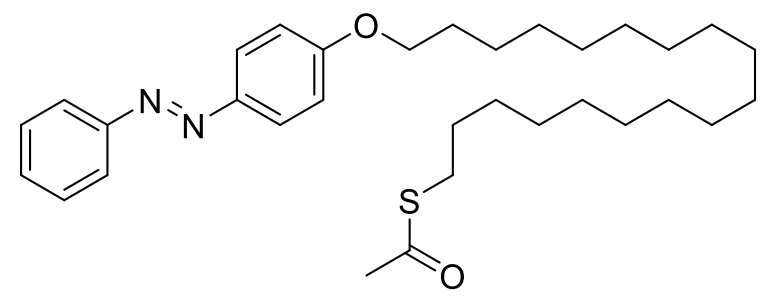

In a flame dried three neck round flask, compound 9 (0.224 g, $0.5 \mathrm{mmol}, 1$ eq.) and azobisisobutyronitrile (AIBN) (0.172 g, $1.05 \mathrm{mmol}, 2.1$ eq.) were dissolved in $5 \mathrm{ml}$ of dry toluene. Thioacetic acid $(0.080 \mathrm{~g}, 1.05 \mathrm{mmol}, 2.1 \mathrm{eq}$.) was added to the mixture dropwise 
under stirring. After addition the mixture was refluxed for 6 hours. The reaction progress was monitored by TLC. Then the flask was cooled down to room temperature. The solution was diluted by $30 \mathrm{ml}$ of toluene, washed with saturated $\mathrm{NaHCO}_{3}$ solution in water $(30 \mathrm{ml})$ and water $(30 \mathrm{ml} \times 2)$. The organic phase was collected and dried over $\mathrm{MgSO}_{4}$. The solvent was evaporated, the residues were purified by column chromatography on silica gel (ethyl acetate : hexane $=1: 40$ ). The product was collected as orange solid, $0.218 \mathrm{~g}$, yield $83 \%$.

${ }^{1} \mathrm{H}$ NMR $\left(400 \mathrm{MHz}, \mathrm{CDCl}_{3}\right) \delta=1.25\left(\mathrm{br}, 30 \mathrm{H}, \mathrm{C}-\left(\mathrm{CH}_{2}\right)_{15}-\mathrm{C}\right), 1.82(\mathrm{dd}, J=8.4,6.4 \mathrm{~Hz}, 2 \mathrm{H}$, O-C-CH$), 2.32\left(\mathrm{~s}, 3 \mathrm{H},-\mathrm{COCH}_{3}\right), 2.86\left(\mathrm{t}, J=7.3 \mathrm{~Hz}, 2 \mathrm{H}, \mathrm{S}-\mathrm{CH}_{2}\right), 4.04(\mathrm{t}, J=6.6 \mathrm{~Hz}, 2 \mathrm{H}$, O-CH 2$), 7.00$ (d, J=8.9 Hz, 2H, Ar-H), 7.44 (m, 1H, Ar-H), 7.50 (m, 2H, Ar-H), 7.89 ppm (m, 4H, Ar-H).

${ }^{13} \mathrm{C}$ NMR $\left(100 \mathrm{MHz}, \mathrm{CDCl}_{3}\right) \delta=13.97,22.53,25.85,28.66,28.96,29.00,29.02,29.20$, $29.22,29.32,29.33,29.41,29.44,29.48,29.50,29.52,30.48,31.77,68.19,114.50,122.34$, $124.56,128.83,130.10,146.65,152.60,161.53,195.93$ ppm.

TOF MS ES+ $(\mathrm{M}+\mathrm{Na})$ Calculated for $\left[\mathrm{C}_{32} \mathrm{H}_{48} \mathrm{~N}_{2} \mathrm{OSNa}^{+}\right]: 547.3329$, found 547.0181.

\section{8-(4-(phenyldiazenyl)phenoxy)octadecane-1-thiol (3)}<smiles>SCCCCCCCCCCCCCCCCCCCCCOc1ccc(N=Nc2ccccc2)cc1</smiles>

Compound 15 (0.104 g, $0.25 \mathrm{mmol}, 1$ eq. $)$ was placed in a flame dried three neck round flask. Then $20 \mathrm{ml}$ of dry $\mathrm{MeOH}$ was added, followed by $3 \mathrm{ml}$ of $\mathrm{HCl}$ in $\mathrm{MeOH}$ solution (1 M). The 
mixture was refluxed for 7 hours. Then the solution was cooled down to room temperature. Excess of $\mathrm{HCl}$ was neutralized with $\mathrm{NaOH}$ in water solution $(1 \mathrm{M})$. The mixture was dissolved in $30 \mathrm{ml}$ of dichloromethane and washed with water $(30 \mathrm{ml} \times 2)$. The organic phase was collected and dried over $\mathrm{MgSO}_{4}$. Product was isolated by silica preparative TLC plate (ethyl acetate : hexane $=1: 50$ ) as orange solid, $0.017 \mathrm{~g}$, yield $14 \%$.

${ }^{1} \mathrm{H}$ NMR $\left(400 \mathrm{MHz}, \mathrm{CDCl}_{3}\right) \delta=1.24-1.38\left(\mathrm{br}, 26 \mathrm{H}, \mathrm{C}-\left(\mathrm{CH}_{2}\right)_{13}-\mathrm{C}\right), 1.33(\mathrm{t}, J=7.7 \mathrm{~Hz}, 1 \mathrm{H},-$ SH), 1.48 (m, 2H, O-C-C-CH $\left.{ }_{2}\right), 1.59$ (m, 2H, S-C-CH $), 1.82$ (m, 2H, O-C-CH $)_{2}, 2.52$ (q, $J$ $\left.=7.5 \mathrm{~Hz}, 2 \mathrm{H}, \mathrm{S}-\mathrm{CH}_{2}\right), 4.04\left(\mathrm{t}, J=6.6 \mathrm{~Hz}, 2 \mathrm{H}, \mathrm{O}-\mathrm{CH}_{2}\right), 7.00(\mathrm{~d}, J=9.0 \mathrm{~Hz}, 2 \mathrm{H}, \mathrm{Ar}-\mathrm{H}), 7.43$ (m, 1H, Ar-H), 7.50 (m, 2H, Ar-H), 7.89 ppm (m, 4H, Ar-H).

${ }^{13} \mathrm{C}$ NMR $\left(100 \mathrm{MHz}, \mathrm{CDCl}_{3}\right) \delta=25.14,26.48,28.85,29.55,29.65,29.85,29.99,30.04$, $30.07,30.12,30.15,34.52,53.89,68.82,115.14,122.98,125.19,129.47,130.74,147.28$, $153.23,162.16 \mathrm{ppm}$.

TOF MS ES $+(\mathrm{M}+\mathrm{Na})$ Calculated for $\left[\mathrm{C}_{30} \mathrm{H}_{46} \mathrm{~N}_{2} \mathrm{OSNa}{ }^{+}\right]: 505.3223$, found 505.3236.

\section{1-(4-(allyloxy)phenyl)-2-(4-(tert-butyl)phenyl)diazene (10)}<smiles>C=CCOc1ccc(/N=N/c2ccc(C(C)(C)C)cc2)cc1</smiles>

In a flame-dried three neck round flask, 4-(4-tert-butylphenylazo)phenol (0.254 g, $1 \mathrm{mmol}$, 1 eq.), 3-bromoprop-1-ene ( $0.121 \mathrm{~g}, 1 \mathrm{mmol}, 1$ eq.) and potassium $t$-butoxide $(0.146 \mathrm{~g}, 1.3$ mmol, 1.3 eq.) were dissolved in $5 \mathrm{ml}$ of dry THF. This mixture was stirred and refluxed 
overnight under $\mathrm{N}_{2}$, reaction progress was monitored by TLC. Then the reaction mixture was cooled down to room temperature, the solvent was evaporated. The residues were dissolved in $50 \mathrm{ml}$ of dichloromethane and washed with water $(30 \mathrm{ml} \times 3)$. The organic phase was collected and dried over $\mathrm{MgSO}_{4}$. The dichloromethane was evaporated and the residues were purified by column chromatography on silica gel (ethyl acetate : hexane $=1: 30$ ). The product was collected as orange solid, $0.221 \mathrm{~g}$, yield $75 \%$.

${ }^{1} \mathrm{H}$ NMR $\left(400 \mathrm{MHz}, \mathrm{CDCl}_{3}\right) \delta=1.38\left(\mathrm{~s}, 9 \mathrm{H},-\mathrm{C}-\left(\mathrm{CH}_{3}\right)_{3}\right), 4.62(\mathrm{dt}, J=5.3,1.5 \mathrm{~Hz}, 2 \mathrm{H}, \mathrm{O}-$ $\left.\mathrm{CH}_{2}\right), 5.33\left(\mathrm{dd}, J=10.5,1.4 \mathrm{~Hz}, 1 \mathrm{H}, \mathrm{C}=\mathrm{CH}_{2} \mathrm{Z}\right.$ position), $5.46(\mathrm{dd}, J=17.2,1.6 \mathrm{~Hz}, 1 \mathrm{H}$ $\mathrm{C}=\mathrm{CH}_{2}$ E position), 6.09 (ddt, $\left.J=17.3,10.5,5.3 \mathrm{~Hz}, 1 \mathrm{H},-\mathrm{CH}=\mathrm{C}\right), 7.03(\mathrm{~d}, J=9.0 \mathrm{~Hz}, 2 \mathrm{H}$, Ar-H), $7.52(\mathrm{~d}, J=8.6 \mathrm{~Hz}, 2 \mathrm{H}, \operatorname{Ar}-\mathrm{H}), 7.82(\mathrm{~d}, J=8.7 \mathrm{~Hz}, 2 \mathrm{H}, \operatorname{Ar}-\mathrm{H}), 7.90 \mathrm{ppm}(\mathrm{d}, J=9.0$ $\mathrm{Hz}, 2 \mathrm{H}, \mathrm{Ar}-\mathrm{H})$.

${ }^{13} \mathrm{C} \mathrm{NMR}\left(100 \mathrm{MHz}, \mathrm{CDCl}_{3}\right) \delta=31.11,34.77,68.84,114.73,117.86,122.08,124.36,125.76$, $132.61,147.01,150.50,153.68,160.62 \mathrm{ppm}$.

TOF MS ES $+(\mathrm{M}+\mathrm{H})$ Calculated for $\left[\mathrm{C}_{19} \mathrm{H}_{23} \mathrm{~N}_{2} \mathrm{O}^{+}\right]$: 295.1805, found 295.1762.

\section{3-(4-((4-(tert-butyl)phenyl)diazenyl)phenoxy)propyl-ethanethioate (16)}<smiles>CC(=O)SCCCOc1ccc(/N=N/c2ccc(C(C)(C)C)cc2)cc1</smiles>

In a flame dried three neck round flask, compound $10(0.147 \mathrm{~g}, 0.5 \mathrm{mmol}, 1 \mathrm{eq}$.$) and$ azobisisobutyronitrile (AIBN) (0.172 g, $1.05 \mathrm{mmol}, 2.1$ eq.) were dissolved in $5 \mathrm{ml}$ of dry 
toluene. Thioacetic acid $(0.080 \mathrm{~g}, 1.05 \mathrm{mmol}, 2.1 \mathrm{eq}$.) was added to the mixture dropwise under stirring. After addition the mixture was refluxed for 6 hours. The reaction progress was monitored by TLC. Then the flask was cooled down to room temperature. The solution was diluted by $30 \mathrm{ml}$ of toluene, washed with saturated $\mathrm{NaHCO}_{3}$ solution in water $(30 \mathrm{ml})$ and water $(30 \mathrm{ml} \times 2)$. The organic phase was collected and dried over $\mathrm{MgSO}_{4}$. The solvent was evaporated, the residues were purified by column chromatography on silica gel (ethyl acetate : hexane $=1: 20)$. The product was collected as orange solid, $0.141 \mathrm{~g}$, yield $76 \%$.

${ }^{1} \mathrm{H}$ NMR $\left(400 \mathrm{MHz}, \mathrm{CDCl}_{3}\right) \delta=1.37\left(\mathrm{~s}, 9 \mathrm{H},-\mathrm{C}-\left(\mathrm{CH}_{3}\right)_{3}\right), 2.11(\mathrm{dd}, J=7.0,6.1 \mathrm{~Hz}, 2 \mathrm{H}, \mathrm{C}-$ $\left.\mathrm{CH}_{2}-\mathrm{C}\right), 2.35\left(\mathrm{~s}, 3 \mathrm{H},-\mathrm{COCH}_{3}\right), 3.08\left(\mathrm{t}, J=7.1 \mathrm{~Hz}, 2 \mathrm{H}, \mathrm{C}-\mathrm{CH}_{2}-\mathrm{S}\right), 4.10(\mathrm{t}, J=6.1 \mathrm{~Hz}, 2 \mathrm{H}$, O-CH 2$), 7.00$ (d, $J=9.0 \mathrm{~Hz}, 2 \mathrm{H}, \mathrm{Ar}-\mathrm{H}), 7.52$ (d, $J=8.7 \mathrm{~Hz}, 2 \mathrm{H}, \mathrm{Ar}-\mathrm{H}), 7.85$ (d, $J=8.6 \mathrm{~Hz}$, 2H, Ar-H), 7.95 ppm (d, $J=9.0 \mathrm{~Hz}, 2 \mathrm{H}, \mathrm{Ar}-\mathrm{H})$.

${ }^{13} \mathrm{C}$ NMR $\left(100 \mathrm{MHz}, \mathrm{CDCl}_{3}\right) \delta=25.95,29.36,30.82,31.41,35.14,66.71,114.94,122.50$, $125.25,126.18,146.79,150.11,154.37,161.63,195.86$ ppm.

TOF MS ES+ $(\mathrm{M}+\mathrm{H})$ Calculated for $\left[\mathrm{C}_{21} \mathrm{H}_{27} \mathrm{~N}_{2} \mathrm{O}_{2} \mathrm{~S}^{+}\right]: 371.1788$, found 371.1757.

\section{3-(4-((4-(tert-butyl)phenyl)diazenyl)phenoxy)propane-1-thiol (4)}<smiles>CC(C)(C)c1ccc(/N=N/c2ccc(OCCCS)cc2)cc1</smiles>

Compound 16 (0.111 g, $0.3 \mathrm{mmol}, 1$ eq.) was placed in a flame dried three neck round flask. Then $8 \mathrm{ml}$ of dry $\mathrm{MeOH}$ was added, followed by $1 \mathrm{ml}$ of $\mathrm{HCl}$ in $\mathrm{MeOH}$ solution $(1 \mathrm{M})$. The 
mixture was refluxed for 3 hours. Then the solution was cooled down to room temperature. Excess of $\mathrm{HCl}$ was neutralized with $\mathrm{NaOH}$ in water solution $(1 \mathrm{M})$. The mixture was dissolved in $30 \mathrm{ml}$ of dichloromethane and washed with water $(30 \mathrm{ml} \times 2)$. The organic phase was collected and dried over $\mathrm{MgSO}_{4}$. The solvent was evaporated, the residues were purified by column chromatography on silica gel (ethyl acetate $:$ hexane $=1: 15$ ). The product was collected as orange solid, $0.055 \mathrm{~g}$, yield $56 \%$.

${ }^{1} \mathrm{H}$ NMR $\left(400 \mathrm{MHz}, \mathrm{CDCl}_{3}\right) \delta=1.37\left(\mathrm{~s}, 9 \mathrm{H},-\mathrm{C}-\left(\mathrm{CH}_{3}\right)_{3}\right), 1.40(\mathrm{t}, J=8.1 \mathrm{~Hz}, 1 \mathrm{H},-\mathrm{SH}), 2.12$ (m, 2H, C-CH $-\mathrm{C}), 2.72\left(\mathrm{t}, J=7.1 \mathrm{~Hz}, 2 \mathrm{H}, \mathrm{S}-\mathrm{CH}_{2}\right), 4.16\left(\mathrm{t}, J=5.5 \mathrm{~Hz}, 2 \mathrm{H}, \mathrm{O}-\mathrm{CH}_{2}\right), 7.01$ (d, $J=9.1 \mathrm{~Hz}, 2 \mathrm{H}, \mathrm{Ar}-\mathrm{H}), 7.52(\mathrm{~d}, J=8.6 \mathrm{~Hz}, 2 \mathrm{H}, \mathrm{Ar}-\mathrm{H}), 7.82(\mathrm{~d}, J=8.6 \mathrm{~Hz}, 2 \mathrm{H}, \mathrm{Ar}-\mathrm{H}), 7.90$ $\operatorname{ppm}(\mathrm{d}, J=9.1 \mathrm{~Hz}, 2 \mathrm{H}, \mathrm{Ar}-\mathrm{H})$.

${ }^{13} \mathrm{C}$ NMR $\left(100 \mathrm{MHz}, \mathrm{CDCl}_{3}\right) \delta=23.13,31.75,32.06,35.41,66.96,115.12,122.71,125.04$, $126.41,147.58,151.14,154.30,161.59 \mathrm{ppm}$.

TOF MS ES+ $(\mathrm{M}+\mathrm{H})$ Calculated for $\left[\mathrm{C}_{19} \mathrm{H}_{25} \mathrm{~N}_{2} \mathrm{OS}^{+}\right]$: 329.1682, found 329.1682.

1-(4-(tert-butyl)phenyl)-2-(4-(undec-10-en-1-yloxy)phenyl)diazene (11),<smiles>C=CCCCCCCCCCOc1ccc(/N=N/c2ccc(C(C)(C)C)cc2)cc1</smiles>

In a flame-dried three neck round flask, 4-(4-tert-butylphenylazo)phenol (0.254 g, $1 \mathrm{mmol}$, 
1 eq.), 11-bromoundec-1-ene ( $0.233 \mathrm{~g}, 1 \mathrm{mmol}, 1$ eq. $)$ and potassium $t$-butoxide $(0.146 \mathrm{~g}, 1.3$ mmol, 1.3 eq.) were dissolved in $5 \mathrm{ml}$ of dry THF. This mixture was stirred and refluxed overnight under $\mathrm{N}_{2}$, reaction progress was monitored by TLC. Then the reaction mixture was cooled down to room temperature, the solvent was evaporated. The residues were dissolved in $50 \mathrm{ml}$ of dichloromethane and washed with water $(30 \mathrm{ml} \times 3)$. The organic phase was collected and dried over $\mathrm{MgSO}_{4}$. The dichloromethane was evaporated and the residues were purified by column chromatography on silica gel (ethyl acetate : hexane $=1: 40$ ). The product was collected as orange solid, $0.207 \mathrm{~g}$, yield $51 \%$.

${ }^{1} \mathrm{H}$ NMR $\left(400 \mathrm{MHz}, \mathrm{CDCl}_{3}\right) \delta=1.32\left(\mathrm{br}, 10 \mathrm{H}, \mathrm{C}-\left(\mathrm{CH}_{2}\right)_{5}-\mathrm{C}\right), 1.37$ (s, 9H, -C- $\left.\left(\mathrm{CH}_{3}\right)_{3}\right), 1.48$ (m, 2H, O-C-C-CH$), 1.83\left(\mathrm{~m}, 2 \mathrm{H}, \mathrm{O}-\mathrm{C}-\mathrm{CH}_{2}\right), 2.05\left(\mathrm{q}, J=6.9 \mathrm{~Hz}, 2 \mathrm{H}, \mathrm{C}=\mathrm{C}-\mathrm{CH}_{2}\right), 4.04(\mathrm{t}, J$ $\left.=6.5 \mathrm{~Hz}, 2 \mathrm{H}, \mathrm{O}-\mathrm{CH}_{2}\right), 4.94\left(\mathrm{~m}, 1 \mathrm{H}, \mathrm{C}=\mathrm{CH}_{2} \mathrm{Z}\right.$ position $), 5.00(\mathrm{dd}, J=17.1,1.9 \mathrm{~Hz}, 1 \mathrm{H}$, $\mathrm{C}=\mathrm{CH}_{2}$ E position), $5.82(\mathrm{ddt}, J=16.9,10.2,6.7 \mathrm{~Hz}, 1 \mathrm{H}, \mathrm{C}-\mathrm{CH}=\mathrm{C}), 7.00,(\mathrm{~d}, J=8.9 \mathrm{~Hz}, 2 \mathrm{H}$, Ar-H), 7.52 (d, $J=8.6 \mathrm{~Hz}, 2 \mathrm{H}, \mathrm{Ar}-\mathrm{H}), 7.82(\mathrm{~d}, J=8.4 \mathrm{~Hz}, 2 \mathrm{H}, \operatorname{Ar}-\mathrm{H}), 7.90 \mathrm{ppm}(\mathrm{d}, J=8.9$ $\mathrm{Hz}, 2 \mathrm{H}, \mathrm{Ar}-\mathrm{H})$.

${ }^{13} \mathrm{C}$ NMR $\left(100 \mathrm{MHz}, \mathrm{CDCl}_{3}\right) \delta=26.16,29.07,29.27,29.34,29.52,29.57,29.65,31.44$, $33.96,35.08,68.47,114.28,114.79,122.36,124.70,126.07,139.36,147.10,150.86,153.90$, $161.60 \mathrm{ppm}$.

TOF MS ES+ $(\mathrm{M}+\mathrm{H})$ Calculated for $\left[\mathrm{C}_{27} \mathrm{H}_{39} \mathrm{~N}_{2} \mathrm{O}^{+}\right]$: 407.3057, found 407.3030.

11-(4-((4-(tert-butyl)phenyl)diazenyl)phenoxy)undecyl-ethanethioate (17), 


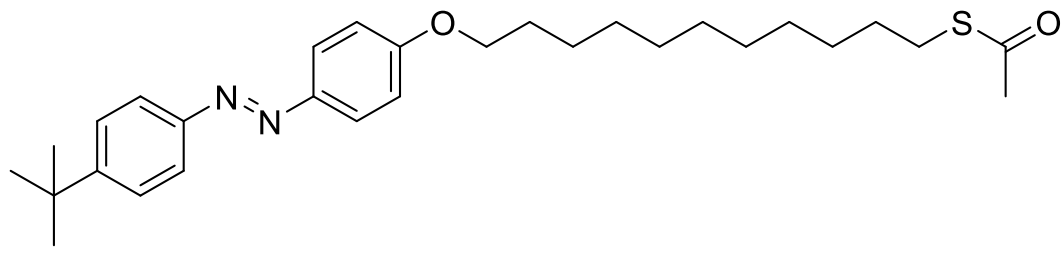

In a flame dried three neck round flask, compound 11 (0.183 g, $0.45 \mathrm{mmol}, 1$ eq.) and azobisisobutyronitrile (AIBN) (0.156 g, $0.95 \mathrm{mmol}, 2.1$ eq.) were dissolved in $5 \mathrm{ml}$ of dry toluene. Thioacetic acid $(0.071 \mathrm{~g}, 0.95 \mathrm{mmol}, 2.1 \mathrm{eq}$. $)$ was added to the mixture dropwise under stirring. After addition the mixture was refluxed for 6 hours. The reaction progress was monitored by TLC. Then the flask was cooled down to room temperature. The solution was diluted by $30 \mathrm{ml}$ of toluene, washed with saturated $\mathrm{NaHCO}_{3}$ solution in water $(30 \mathrm{ml})$ and water $(30 \mathrm{ml} \times 2)$. The organic phase was collected and dried over $\mathrm{MgSO}_{4}$. The solvent was evaporated, the residues were purified by column chromatography on silica gel (ethyl acetate : hexane $=1: 40$ ). The product was collected as orange solid, $0.119 \mathrm{~g}$, yield $55 \%$.

${ }^{1} \mathrm{H}$ NMR $\left(400 \mathrm{MHz}, \mathrm{CDCl}_{3}\right) \delta=1.29\left(\mathrm{~m}, 12 \mathrm{H}, \mathrm{C}-\left(\mathrm{CH}_{2}\right)_{6}-\mathrm{C}\right), 1.37\left(\mathrm{~s}, 9 \mathrm{H},-\mathrm{C}-\left(\mathrm{CH}_{3}\right)_{3}\right), 1.47$ (m, 2H, O-C-C-CH$), 1.56\left(\mathrm{~m}, 2 \mathrm{H}, \mathrm{S}-\mathrm{C}-\mathrm{CH}_{2}\right), 1.81\left(\mathrm{~m}, 2 \mathrm{H}, \mathrm{O}-\mathrm{C}-\mathrm{CH}_{2}\right), 2.32\left(\mathrm{~s}, 3 \mathrm{H},-\mathrm{COCH}_{3}\right)$, $2.86\left(\mathrm{t}, J=7.3 \mathrm{~Hz}, 2 \mathrm{H}, \mathrm{S}-\mathrm{CH}_{2}\right), 4.04\left(\mathrm{t}, J=6.6 \mathrm{~Hz}, 2 \mathrm{H}, \mathrm{O}-\mathrm{CH}_{2}\right), 7.00,(\mathrm{~d}, J=8.9 \mathrm{~Hz}, 2 \mathrm{H}$, Ar-H), 7.52 (d, $J=8.6 \mathrm{~Hz}, 2 \mathrm{H}, \operatorname{Ar}-\mathrm{H}), 7.84(\mathrm{~d}, J=8.6 \mathrm{~Hz}, 2 \mathrm{H}, \operatorname{Ar}-\mathrm{H}), 7.93 \mathrm{ppm}(\mathrm{d}, J=9.0$ $\mathrm{Hz}, 2 \mathrm{H}, \mathrm{Ar}-\mathrm{H})$.

${ }^{13} \mathrm{C}$ NMR $\left(100 \mathrm{MHz}, \mathrm{CDCl}_{3}\right) \delta=26.14,28.96,29.24,29.31,29.47,29.58,29.62,29.65$, $30.80,31.41,35.13,68.59,114.96,122.46,125.31,126.17,146.52,150.06,154.29,162.15$, $196.24 \mathrm{ppm}$.

TOF MS ES+ $(\mathrm{M}+\mathrm{H})$ Calculated for $\left[\mathrm{C}_{29} \mathrm{H}_{43} \mathrm{~N}_{2} \mathrm{O}_{2} \mathrm{~S}^{+}\right]$: 483.3040, found 483.3024. 


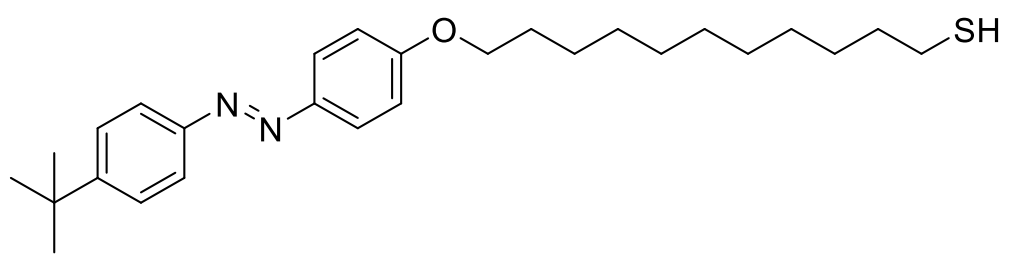

Compound 17 (0.091 g, $0.2 \mathrm{mmol}, 1$ eq.) was placed in a flame dried three neck round flask. Then $7 \mathrm{ml}$ of dry $\mathrm{MeOH}$ was added, followed by $0.8 \mathrm{ml}$ of $\mathrm{HCl}$ in $\mathrm{MeOH}$ solution (1 M). The mixture was refluxed for 3 hours. Then the solution was cooled down to room temperature. Excess of $\mathrm{HCl}$ was neutralized with $\mathrm{NaOH}$ in water solution (1 M). The mixture was dissolved in $30 \mathrm{ml}$ of dichloromethane and washed with water $(30 \mathrm{ml} \times 2)$. The organic phase was collected and dried over $\mathrm{MgSO}_{4}$ The dichloromethane was evaporated and the residues were purified by column chromatography on silica gel (ethyl acetate : hexane $=1$ : 50), product was collected as orange solid, $0.051 \mathrm{~g}$, yield $58 \%$.

${ }^{1} \mathrm{H}$ NMR $\left(400 \mathrm{MHz}, \mathrm{CDCl}_{3}\right) \delta=1.29\left(\mathrm{~m}, 12 \mathrm{H}, \mathrm{C}-\left(\mathrm{CH}_{2}\right)_{6}-\mathrm{C}\right), 1.33(\mathrm{t}, J=7.7 \mathrm{~Hz}, 1 \mathrm{H},-\mathrm{SH})$, 1.37 (s, 9H, -C- $\left.\left(\mathrm{CH}_{3}\right)_{3}\right), 1.47$ (m, 2H, O-C-C-CH C-CH 2$), 2.52\left(\mathrm{q}, J=7.4 \mathrm{~Hz}, 2 \mathrm{H}, \mathrm{S}-\mathrm{CH}_{2}\right), 4.05\left(\mathrm{t}, J=6.5 \mathrm{~Hz}, 2 \mathrm{H}, \mathrm{O}-\mathrm{CH}_{2}\right), 7.01(\mathrm{~d}, J=9.0$ Hz, 2H, Ar-H), 7.52 (d, $J=8.6$ Hz, 2H, Ar-H), 7.88 (d, J=8.6 Hz, 2H, Ar-H), 7.98 ppm (d, $J=8.9 \mathrm{~Hz}, 2 \mathrm{H}, \mathrm{Ar}-\mathrm{H})$.

${ }^{13} \mathrm{C}$ NMR $\left(100 \mathrm{MHz}, \mathrm{CDCl}_{3}\right) \delta=24.81,26.13,28.52,28.66,29.21,29.30,29.36,29.38$, $29.49,29.51,29.64,29.66,31.40,34.19,35.16,39.33,68.64,115.05,122.51,125.67,126.23$, $146.17,149.62,154.51,162.46 \mathrm{ppm}$. 
TOF MS ES+ $(\mathrm{M}+\mathrm{H})$ Calculated for $\left[\mathrm{C}_{27} \mathrm{H}_{41} \mathrm{~N}_{2} \mathrm{OS}^{+}\right]$: 441.2934, found 441.2930.

\section{1-(4-(tert-butyl)phenyl)-2-(4-(octadec-17-en-1-yloxy)phenyl)diazene (12),}

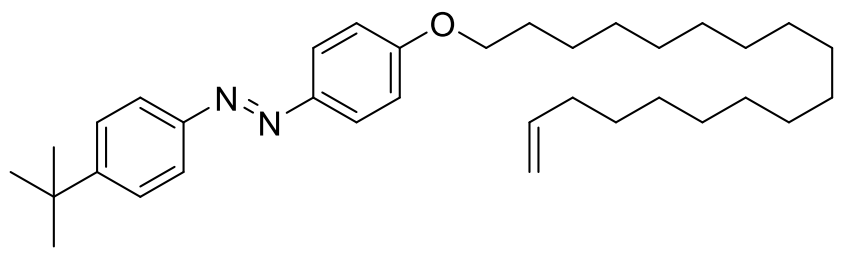

In a flame-dried three neck round flask, 4-(4-tert-butylphenylazo)phenol (0.254 g, $1 \mathrm{mmol}$, 1 eq.), 18-bromooctadec-1-ene (0.331 g, $1 \mathrm{mmol}, 1$ eq.) and potassium $t$-butoxide ( $0.146 \mathrm{~g}$, $1 \mathrm{mmol}, 1.3$ eq.) were dissolved in $7.5 \mathrm{ml}$ of dry THF. This mixture was stirred and refluxed overnight under $\mathrm{N}_{2}$, reaction progress was monitored by TLC. Then the reaction mixture was cooled down to room temperature, the solvent was evaporated. The residues were dissolved in $50 \mathrm{ml}$ of dichloromethane and washed with water $(30 \mathrm{ml} \times 3)$. The organic phase was collected and dried over $\mathrm{MgSO}_{4}$. The dichloromethane was evaporated and the residues were purified by column chromatography on silica gel (ethyl acetate : hexane $=1: 50$ ). The product was collected as orange solid, $0.312 \mathrm{~g}$, yield $62 \%$.

${ }^{1} \mathrm{H}$ NMR $\left(400 \mathrm{MHz}, \mathrm{CDCl}_{3}\right) \delta=1.27\left(\mathrm{br}, 24 \mathrm{H}, \mathrm{C}-\left(\mathrm{CH}_{2}\right)_{12}-\mathrm{C}\right), 1.37\left(\mathrm{~s}, 9 \mathrm{H},-\mathrm{C}-\left(\mathrm{CH}_{3}\right)_{3}\right), 1.47$ (m, 2H, O-C-C-CH$), 1.81\left(\mathrm{~m}, 2 \mathrm{H}, \mathrm{O}-\mathrm{C}-\mathrm{CH}_{2}\right), 2.04\left(\mathrm{~m}, 2 \mathrm{H}, \mathrm{C}=\mathrm{C}-\mathrm{CH}_{2}\right), 4.03(\mathrm{t}, J=6.6 \mathrm{~Hz}$, 2H, O- $\mathrm{CH}_{2}$ ), 4.95 (dd, $J=10.5,1.4 \mathrm{~Hz}, 1 \mathrm{H}, \mathrm{C}=\mathrm{CH}_{2} \mathrm{Z}$ position), 4.99 (dd, $J=17.2,1.6 \mathrm{~Hz}$, 1H, $\mathrm{C}=\mathrm{CH}_{2}$ E position), $5.82(\mathrm{ddt}, J=16.9,10.2,6.7 \mathrm{~Hz}, 1 \mathrm{H}, \mathrm{C}-\mathrm{CH}=\mathrm{C}), 7.00(\mathrm{~d}, J=9.0 \mathrm{~Hz}$, 2H, Ar-H), 7.52 (d, $J=8.6 \mathrm{~Hz}, 2 \mathrm{H}, \mathrm{Ar}-\mathrm{H}), 7.81(\mathrm{~d}, J=8.6 \mathrm{~Hz}, 2 \mathrm{H}, \mathrm{Ar}-\mathrm{H}), 7.89 \mathrm{ppm}(\mathrm{d}, J=$ $9.0 \mathrm{~Hz}, 2 \mathrm{H}, \mathrm{Ar}-\mathrm{H})$. 
${ }^{13} \mathrm{C}$ NMR $\left(100 \mathrm{MHz}, \mathrm{CDCl}_{3}\right) \delta=26.16,29.10,29.31,29.35,29.54,29.67,29.72,29.75$, $29.78,29.81,29.83,31.09,31.44,33.98,35.08,68.48,114.22,114.79,122.36,124.69$, $126.07,139.42,147.09,150.85,153.89,161.61 \mathrm{ppm}$.

TOF MS ES+ $(\mathrm{M}+\mathrm{Na})$ Calculated for $\left[\mathrm{C}_{34} \mathrm{H}_{53} \mathrm{~N}_{2} \mathrm{O}^{+}\right]$: 505.4152, found 505.4150.

\section{8-(4-((4-(tert-butyl)phenyl)diazenyl)phenoxy)octadecyl-ethanethioate (18),}

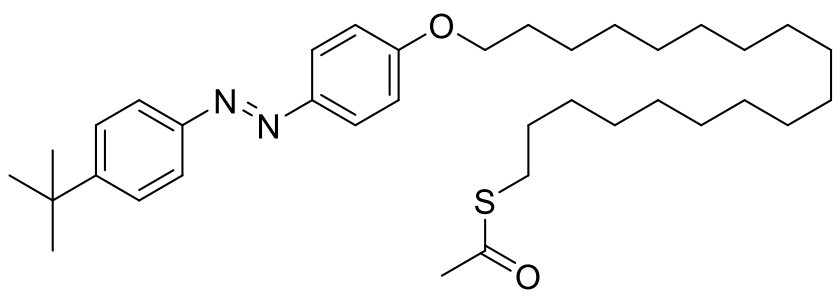

In a flame dried three neck round flask, compound $12(0.126 \mathrm{~g}, 0.25 \mathrm{mmol}, 1$ eq.) and azobisisobutyronitrile (AIBN) (0.086 g, $0.525 \mathrm{mmol}, 2.1$ eq.) were dissolved in $5 \mathrm{ml}$ of dry toluene. Thioacetic acid $(0.040 \mathrm{~g}, 0.525 \mathrm{mmol}, 2.1 \mathrm{eq}$.) was added to the mixture dropwise under stirring. After addition the mixture was refluxed for 6 hours. The reaction progress was monitored by TLC. Then the flask was cooled down to room temperature. The solution was diluted by $30 \mathrm{ml}$ of toluene, washed with saturated $\mathrm{NaHCO}_{3}$ solution in water $(30 \mathrm{ml})$ and water $(30 \mathrm{ml} \times 2)$. The organic phase was collected and dried over $\mathrm{MgSO}_{4}$. The solvent was evaporated, the residues were purified by column chromatography on silica gel (ethyl acetate : hexane $=1: 40$ ). The product was collected as orange solid, $0.117 \mathrm{~g}$, yield $81 \%$.

${ }^{1} \mathrm{H}$ NMR $\left(400 \mathrm{MHz}, \mathrm{CDCl}_{3}\right) \delta=1.26\left(\mathrm{~m}, 28 \mathrm{H}, \mathrm{C}-\left(\mathrm{CH}_{2}\right)_{14}-\mathrm{C}\right), 1.37\left(\mathrm{~s}, 9 \mathrm{H}-\mathrm{C}-\left(\mathrm{CH}_{3}\right)_{3}\right), 1.49$ (m, 2H, S-C-CH $), 1.82$ (m, 2H, O-C-CH ${ }_{2}$ ), 2.32 (s, 3H, - $\left.\mathrm{COCH}_{3}\right), 2.86$ (m, 2H, S-CH $), 4.03$ (t, $\left.J=6.6 \mathrm{~Hz}, 2 \mathrm{H}, \mathrm{O}-\mathrm{CH}_{2}\right), 6.99$ (d, $\left.J=9.0 \mathrm{~Hz}, 2 \mathrm{H}, \mathrm{Ar}-\mathrm{H}\right), 7.51(\mathrm{~d}, J=8.6 \mathrm{~Hz}, 2 \mathrm{H}, \mathrm{Ar}-\mathrm{H})$, 
$7.81(\mathrm{~d}, J=8.6 \mathrm{~Hz}, 2 \mathrm{H}, \mathrm{Ar}-\mathrm{H}), 7.89 \mathrm{ppm}(\mathrm{d}, J=8.9 \mathrm{~Hz}, 2 \mathrm{H}, \mathrm{Ar}-\mathrm{H})$.

${ }^{13} \mathrm{C}$ NMR $\left(100 \mathrm{MHz}, \mathrm{CDCl}_{3}\right) \delta=14.28,26.17,28.98,29.28,29.32,29.35,29.54,29.65$, $29.73,29.75,29.79,29.82,29.84,30.80,31.44,35.08,68.50,114.80,122.36,124.70,126.08$, $128.37,129.18,147.10,150.86,153.90,161.61,196.25 \mathrm{ppm}$.

TOF MS ES+ $(\mathrm{M}+\mathrm{H})$ Calculated for $\left[\mathrm{C}_{36} \mathrm{H}_{57} \mathrm{~N}_{2} \mathrm{O}_{2} \mathrm{~S}^{+}\right]: 581.4135$, found 581.4142.

\section{8-(4-((4-(tert-butyl)phenyl)diazenyl)phenoxy)octadecane-1-thiol (6)}

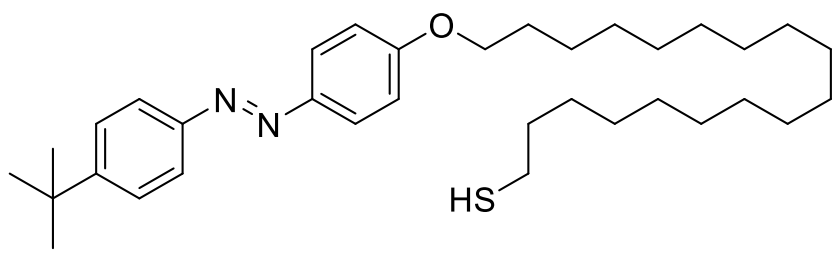

Compound 18 (0.067 g, $0.115 \mathrm{mmol}, 1$ eq.) was placed in a flame dried three neck round flask. Then $15 \mathrm{ml}$ of dry $\mathrm{MeOH}$ was added, followed by $2 \mathrm{ml}$ of $\mathrm{HCl}$ in $\mathrm{MeOH}$ solution (1 M). The mixture was refluxed for 7 hours. Then the solution was cooled down to room temperature. Excess $\mathrm{HCl}$ was neutralized with $\mathrm{NaOH}$ in water solution $(1 \mathrm{M})$. The mixture was dissolved in $30 \mathrm{ml}$ of dichloromethane and washed with water $(30 \mathrm{ml} \times 2)$. The organic phase was collected and dried over $\mathrm{MgSO}_{4}$. Product was isolated by silica preparative TLC plate (ethyl acetate : hexane $=1: 40$ ) as orange solid, $0.028 \mathrm{~g}$, yield $45 \%$.

${ }^{1} \mathrm{H}$ NMR $\left(400 \mathrm{MHz}, \mathrm{CDCl}_{3}\right) \delta=1.26\left(\mathrm{br}, 28 \mathrm{H}, \mathrm{C}-\left(\mathrm{CH}_{2}\right)_{14}-\mathrm{C}\right), 1.33(\mathrm{t}, J=7.7 \mathrm{~Hz}, 1 \mathrm{H},-\mathrm{SH})$, $1.37\left(\mathrm{~s}, 9 \mathrm{H},-\mathrm{C}-\left(\mathrm{CH}_{3}\right)_{3}\right), 1.46\left(\mathrm{~m}, 2 \mathrm{H}, \mathrm{S}-\mathrm{C}-\mathrm{CH}_{2}\right), 1.81\left(\mathrm{~m}, 2 \mathrm{H}, \mathrm{O}-\mathrm{C}-\mathrm{CH}_{2}\right), 2.52(\mathrm{q}, J=7.5 \mathrm{~Hz}$, 2H, S-CH $), 4.04\left(\mathrm{t}, J=6.6 \mathrm{~Hz}, 2 \mathrm{H}, \mathrm{O}-\mathrm{CH}_{2}\right), 7.00$ (d, $\left.J=9.0 \mathrm{~Hz}, 2 \mathrm{H}, \mathrm{Ar}-\mathrm{H}\right), 7.51(\mathrm{~d}, J=8.7$ $\mathrm{Hz}, 2 \mathrm{H}, \mathrm{Ar}-\mathrm{H}), 7.83$ (d, $J=8.7 \mathrm{~Hz}, 2 \mathrm{H}, \mathrm{Ar}-\mathrm{H}), 7.91 \mathrm{ppm}(\mathrm{d}, J=9.0 \mathrm{~Hz}, 2 \mathrm{H}, \mathrm{Ar}-\mathrm{H})$. 
${ }^{13} \mathrm{C}$ NMR $\left(100 \mathrm{MHz}, \mathrm{CDCl}_{3}\right) \delta=24.81,26.12,28.53,28.68,29.23,29.27,29.37,29.40$, $29.51,29.67,29.71,29.74,29.80,29.82,29.83,29.85,31.36,34.20,35.22,39.36,68.82$, $115.24,122.63,126.35,145.48,148.64,154.97,163.13 \mathrm{ppm}$.

TOF MS ES+ $(\mathrm{M}+\mathrm{H})$ Calculated for $\left[\mathrm{C}_{34} \mathrm{H}_{55} \mathrm{~N}_{2} \mathrm{OS}^{+}\right]$: 539.4030, found 539.4041.

\subsubsection{UV/Vis absorption spectrum of the ligands in chloroform solution}

a)

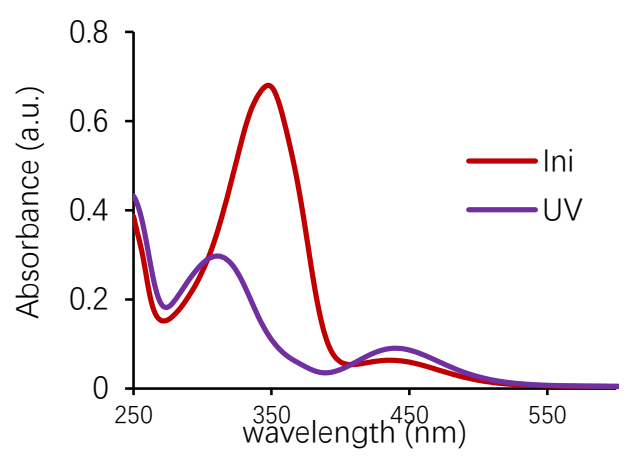

b)

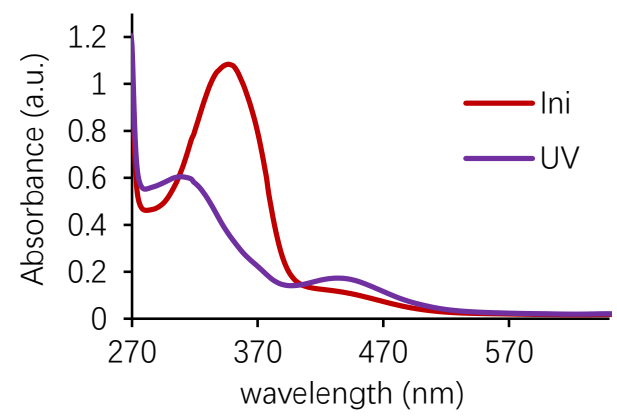


c)

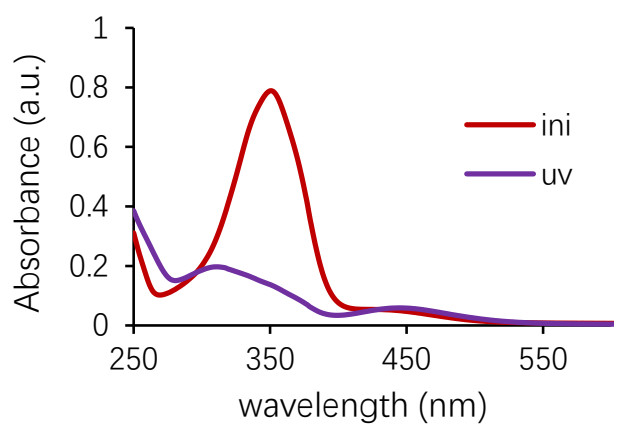

d)

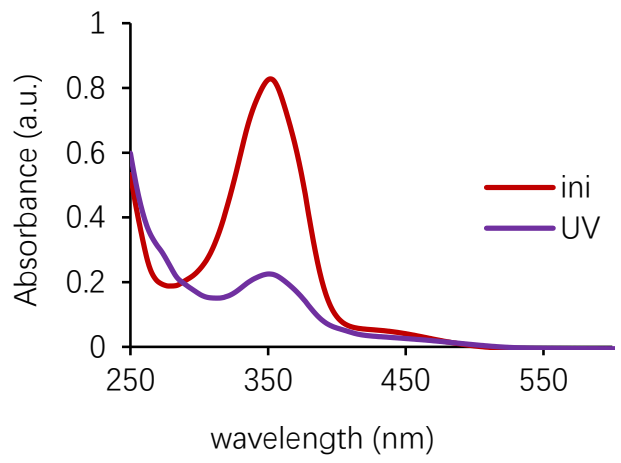

e)

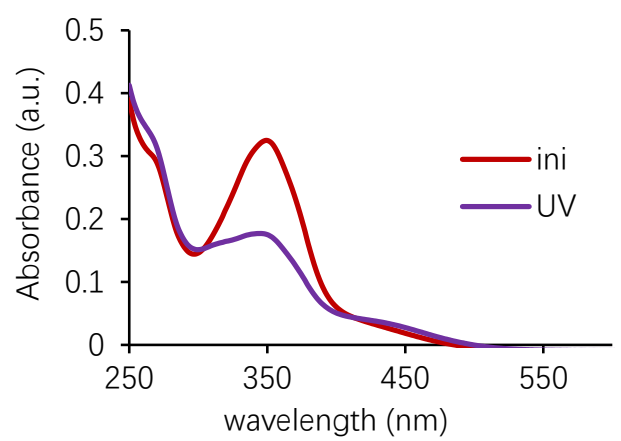

Figure S6.2. UV/Vis spectrum of the ligands in chloroform solution. a) ligand 2, $\mathrm{c}=50 \mu \mathrm{M}, \mathrm{b}$ ) ligand 3, $\mathrm{c}=70 \mu \mathrm{M}, \mathrm{c}$ ) ligand $4, \mathrm{c}=50 \mu \mathrm{M}, \mathrm{d})$ ligand $5, \mathrm{c}=50 \mu \mathrm{M}, \mathrm{e})$ ligand $6, \mathrm{c}=20 \mu \mathrm{M}$. UV irradiation: $\lambda$ $=365 \mathrm{~nm}, 1 \mathrm{~min}$. 


\subsubsection{Lateral size of the nanoplatelets}

Average lateral size of the nanoplatelets were measured by 20 individual dispersed nanoplatelets from each TEM images.

Table S6.1. Lateral size of the nanoplatelets.

\begin{tabular}{|c|c|c|c|}
\hline \multicolumn{2}{|c|}{$\begin{array}{c}\text { nanoplatelets size according to TEM } \\
\text { image of Figure 6.3a }\end{array}$} & \multicolumn{2}{|c|}{$\begin{array}{c}\text { nanoplatelets size according to TEM } \\
\text { image of Figure } \mathbf{6 . 4 a}\end{array}$} \\
\hline entry & $\operatorname{area}\left(\mathrm{nm}^{2}\right)$ & entry & $\operatorname{area}\left(\mathrm{nm}^{2}\right)$ \\
\hline 1 & 496.077 & 1 & 394.323 \\
\hline 2 & 476.000 & 2 & 479.016 \\
\hline 3 & 476.837 & 3 & 371.469 \\
\hline 4 & 431.663 & 4 & 386.676 \\
\hline 5 & 401.547 & 5 & 435.461 \\
\hline 6 & 359.719 & 6 & 398.455 \\
\hline 7 & 414.095 & 7 & 342.330 \\
\hline 8 & 367.248 & 8 & 480.422 \\
\hline 9 & 409.912 & 9 & 440.955 \\
\hline 10 & 480.183 & 10 & 396.389 \\
\hline 11 & 356.373 & 11 & 374.853 \\
\hline 12 & 335.459 & 12 & 421.265 \\
\hline 13 & 477.673 & 13 & 443.152 \\
\hline 14 & 550.454 & 14 & 408.519 \\
\hline 15 & 542.925 & 15 & 400.476 \\
\hline 16 & 404.056 & 16 & 576.454 \\
\hline 17 & 325.420 & 17 & 367.733 \\
\hline 18 & 558.819 & 18 & 392.302 \\
\hline 19 & 470.144 & 19 & 395.598 \\
\hline 20 & 397.364 & 20 & 451.283 \\
\hline average & 436.598 & average & 417.857 \\
\hline
\end{tabular}




\subsection{References}

[1] C. Burda, X. B. Chen, R. Narayanan, M. A. El-Sayed, Chem. Rev., 2005, 105, 1025.

[2] J. N. Anker, W. P. Hall, O. Lyandres, N. C. Shah, J. Zhao, R. P. van Duyne, Nature Mater, 2008, 7, 442 .

[3] D. V. Talapin, J-S. Lee, M. V. Kovalenko, E. V. Shevchenko, Chem. Rev., 2010, 110, 389.

[4] K. D. Gilroy, A. Ruditskiy, H-C. Peng, D. Qin, Y. Xia, Chem. Rev., 2016, 116, 10414.

[5] L. Wu, A. Mendoza-Garcia, Q. Li, S. Sun, Chem. Rev., 2016, 116, 10473.

[6] M. Li, H. Schnablegger, S. Mann, Nature, 1999, 402, 393.

[7] A. K. Boal, F. Ilhan, J. E. DeRouchey, T. Thurn-Albrecht, T. P. Russell, V. M. Rotello, Nature, 2001, 404, 746.

[8] R. Narayanan, M. A. El-Sayed, J. Phys. Chem. B, 2005, 109, 12663.

[9] W. M. Gelbart, R. P. Sear. J. R. Heath, S. Chaney, Farad. Disc., 1999, 122, 299.

[10] G. M. Whitesides, B. Grzybowski, Science, 2002, 295, 2418.

[11] E. Rabani, D. R. Reichman, P. L. Geissler, L. E. Brus, Nature, 2003, 426, 271.

[12] M. Grzelczak, J. Vermant, E. M. Furst, L. M. Liz-Marzán, ACS Nano, 2010, 4, 3591.

[13] R. Klajn, J. F. Stoddart, B. A. Grzybowski, Chem. Soc. Rev., 2010, 39, 2203.

[14] R. Mikami, M. Taguchi, K. Yamada, K. Suzuki, O. Sato and Y. Einaga, Angew. Chem., Int. Ed., 2004, 43, 6135 .

[15] M. Suda, N. Kameyama, A. Ikegami, M. Suzuki, N. Kawamura, Y. Einaga, Polyhedron, 2009, 28, 1868.

[16] M. Suda, M. Nakagawa, T. Iyoda, Y. Einaga, J. Am. Chem. Soc., 2007, 129, 5538.

[17] R. Klajn, K. J. M. Bishop, B. A. Grzybowski, Proc. Natl. Acad. Sci. U. S. A., 2006, 104, 10305 .

[18] M. Ueda, N. Fukushima, K. Kudo, K. Ichimura, J. Mater. Chem., 1997, 7, 641.

[19] S. Neri, S. G. Martin, C. Pezzato, L. J. Prins, J. Am. Soc. Chem., 2017, 139, 1794.

[20] H. Zhao, S. Sen, T. Udayabhaskararao, M. Sawczyk, K. Kučanda, D. Manna, P. K. Kundu, J-W. Lee, P. Král, R. Klajn, Nature Nanotech., 2016, 11, 82.

[21] A, Zep, M. M. Wojcik, W. Lewandowski, K. Sitkowska, A. Prominski, J. Mieczkowski, D. Pociecha, E. Gorecka, Angew. Chem., 2014, 126, 13945.

[22] C. Xue, J. Xiang, H. Nemati, H. K. Bisoyi, K. Gutierrez-Cuevas, L. Wang, M. Gao, S. Zhou, D-K. Yang, O. D. Lavrentovich, A. Urbas, Q. Li, ChemPhysChem, 2015, 16, 1852.

[23] R. Klajn, P. J. Wesson, K. J. M. Bishop, B. A. Grzybowski, Angew. Chem. Int. Ed., 2009, 48,7035 . 
[24] S. Bonacchi, A. Cantelli, G. Guidetti, M. Calvaresi, J. Manzi, L. Gabrielli, F. Ramadori, A. Gambarin, F. Mancin, M. Montalti, Angew. Chem. Int. Ed., 2016, 55, 11064.

[25] X. Michalet, F. F. Pinaud, L. A. Bentolila, J. M. Tsay, S. Doose, J. J. Li, G. Sunderesan, A. M. Wu, S. S. Gambhir, S. Weiss, Science, 2005, 307, 538.

[26] D. R. Baker, P. V. Kamat, Langmuir, 2010, 26, 11272.

[27] V. L. Colvin, M. C. Schlamp, A. P. Allvisatos, Nature, 1994, 370, 354.

[28] B. S. Mashford, M. Stevenson, Z. Popovic, C. Hamilton, Z. Zhou, C. Breen, J. Steckel, V. Bulovic, M. Bawendi, S. Coe-Sullivan, P. T. Kazlas, Nature Photonics, 2013, 7, 407.

[29] H. S. Jang, H. Yang, S. W. Kim, J. Y. Han, S-G. Lee, D. Y. Jeon, Adv. Mater., 2008, 20, 2696.

[30] J. M. Pietryga, Y-S. Park, J. Lim, A. F. Fidler, W. K. Bae, S. Brovelli, V. I. Klimov, Chem. Rev., 2016, 116, 10513.

[31] D. V. Talapin, J. Steckel, MRS Bull., 2013, 38, 685.

[32] A. P. Alivisatos, Science, 1996, 271, 933.

[33] A. M. Smith, S. Nie, Acc. Chem. Res., 2010, 43, 190.

[34] Y. E. Panfil, M. Oded, U. Banin, Angew. Chem. Int. Ed., accepted article, DOI: 10.1002/anie.201708510.

[35] S. Ithurria, B. Dubertret, J. Am. Chem. Soc., 2008, 130, 16504.

[36] E. Lhuillier, S. Oedetti, S. Ithurria, B. Nahal, H. Heuclin, B. Dubertret, Acc. Chem. Res., 2015, 48, 22.

[37] M. Nasilowski, B. Mahler, E. Lhuillier, S. Ithurria, B. Dubertret, Chem. Rev., 2016, 116, 10934.

[38] C. She, L. Fedin, D. S. Dolzhnikov, A. Demortière, R. D. Schaller, M. Pelton, D. V. Talapin, Nano Lett., 2014, 14, 2772.

[39] C. She, I. Fedin, D. S. Dolzhnikov, P. D. Dahlberg, G. S. Engel, R. D. Schaller, D. V. Talapin, ACS Nano, 2015, 9, 9475.

[40] B. Guzelturk, M. Olutas, S. Delikanli, Y. Kelestemur, O. Erdem, H. V. Demir, Nanoscale, 2015, 7, 2545.

[41] F. Zhang, S. Wang, L. Wang, Q. Lin, H. Shen, W. Cao, C. Yang, H. Wang, L. Yu, Z. Du, J. Xue, L. S. Li, Nanoscale, 2016, 8, 12182.

[42] M. R. Joins, R. J. Macfarlane, A. E. Prigodlich, P. C. Patel, C. A. Mirkin, J. Am. Chem. Soc., 2011, 133, 18865.

[43] S. C. Glotzer, Nature, 2012, 481, 450.

[44] B. Abécassis, M. D. Tessier, P. Davidson, B. Dubertret, Nano Lett., 2014, 14, 710. 
[45] S. Jana, P. Davidson, B. Abécassis, Angew. Chem. Int. Ed., 2016, 55, 9371.

[46] B. Guzelturk, O. Erdem, M. Olutas, Y. Kelestemur, H. V. Demir, ACS Nano, 2014, 8, 12524.

[47] X. Peng, T. E. Wilson, A. P. Alivisatos, P. G. Schultz, Angew. Chem. Int. Ed. Engl. 1997, 36,145 .

[48] J. Owen, Science, 2015, 347, 615.

[49] N. C. Anderson, M. P. Hendricks, J. J. Choi, J. S. Owen, J. Am. Chem. Soc., 2013, 135, 18536.

[50] R. R. Knauf, J. C. Lennox, J. L. Dempsey, Chem. Mater., 2016, $28,4762$.

[51] H. Akiyama, K. Tamada, J. Nagasawa, K. Abe, T. Tamaki, J. Phys. Chem. B, 2003, 107, 130 .

[52] E. Lhuillier, J-F. Dayen, D. O. Thomas, A. Robin, S. Ithurria, H. Aubin, B. Dubertret, Phys. Status Solidi C, 2016, 13, 526.

[53] A. Yeltik, S. Delikanli, M. Olutasm Y. Kelestemur, B, Guzelturk, H. V. Demir, J. Phys. Chem. C, 2015, 119, 26768.

[54] K. E. Snell, J-Y. Mevellec, B. Humbert, F. Lagugné-Labarthet, E. Ishow, ACS appl. Mater. Interfaces, 2015, 7, 1932.

[55] D. Manna, T. Udayabhaskararao, H. Zhao, R. Klajn, Angew. Chem. Int. Ed., 2015, 54, 12394.

[56] A. Shukurovm D, D, Nasirdinov, A. G. Makhsumov, N. N. Edgarov, Zhurnal Obshchei Khimii, 1986, 56, 2579.

[57] K. Peng, I. Tomatsu, A. Kros, Chem. Commun., 2010, 46, 4094.

[58] S. Moeller, U. Pliquett, C. Hoffmann, RSC Advances, 2012, 2, 4792.

[59] A. Köhntopp, M. Dittner, F. Temps. J. Phys. Chem. Lett., 2016, 7, 1088. 


\section{Summary}

Interfacing molecular photo-switches with supramolecular systems allows developing dynamic smart materials with photo-responsive functions, provided that the energy input from photo-illumination can be converted into different forms, and amplified across multiple length scales. In this thesis, we focus on azobenzene photo-switches as dynamic building blocks, in view of their easy synthesis and functionalization, high extinction coefficient and quantum yields, as well as the changes on both molecular geometry and dipole moment during the photo-isomerization process. A main challenge remaining with the lack of cisisomer stability, which limits the application by losing the encoded cis- state information. Meanwhile UV-light irradiation is unfavored in bio-environment due to the tissue harmful issue. Thus, we started with study on fluorinated azobenzenes, for their application in supramolecular materials for kinetic control and visible-light controlled switching.

In Chapter 1, we present a brief overview of commonly used molecular photo-switches.

In Chapter 2, we discuss recent designing and engineering strategies that aim at developing cis- stable azobenzenes. These strategies include ortho-fluorination, orthomethoxylation, azoheteroarene, hydrazone and azobenzene- $\mathrm{BF}_{2}$ conjugation, cyclic azobenzene.

In Chapter 3, two strategies, ortho-fluorination and axial chirality, are combined to in azobenzene-based dynamic dopants for liquid crystals. In both isotropic solution and in the liquid crystalline phase, these chiral dopants display better cis-form stability than their nonfluorinated counterparts. In the liquid crystalline phase, long-lived helical structures with cis- 
state dopant could be created by irradiation. However, the fluorinated dopants remain associated with moderate helical twisting powers and variations thereof, upon photoisomerization in cholesteric liquid crystals. This cholesteric liquid crystal will be potentially applied into optical display devices or photo-responsive actuators.

In Chapter 4, we investigate the host-guest chemistry of ortho-fluorinated azobenzenes in two common host cavities: $\beta$-cyclodextrin and cucurbit[8]uril. In the presence of both host cavities, ortho-fluorinated azobenzenes form stable supramolecular complexes. In the transform, binding to $\beta$-cyclodextrin is favored, whereas in its cis-form complexation to cucurbit[8] uril is favored. Upon light-triggered photo-isomerization, we show that the photoswitch can be transferred from one host cavity to the other, reversibly.

In Chapter 5, the host-guest chemistry of ortho-fluorinated azobenzenes was involved in the design of multivalent architectures, with the final aim to build dynamic supramolecular nanoparticles that would display photo-triggered self-assembly. In the bi-componenet system composed by fluorinated azobenzenes and $\beta$-cyclodextrins, the binding constant is of the same order of magnitude in both the trans- and the cis-state, thus the supramolecular nanoparticles show no response to photo-isomerization. Cucurbit[8]uril was then introduced into the system to bind with cis-azobenzene we anticipated that the supramolecular nanoparticles would dis-assemble by competitive complexation. However, the binding between the azobenzene and $\beta$-cyclodextrin is enhanced by multivalency effects, thus the cucurbit[8]uril could not compete against $\beta$-cyclodextrin in the host guest complexation, and the supramolecular system remained non photo-responsive.

Another broadly interested question nowadays is the stimuli controlled dynamic assembly 
and dispersion. In Chapter 6, azobenzene-based photo-switches are employed as photoresponsive ligands for nanoplatelets of an inorganic semi-conductor. Upon irradiation, the dipole-dipole interaction between cis-azobenzenes induced face-to-face self-assembly of the nanoplatelets, into supra-particular ribbons. The next step investigation will be focused on mechanism of the ribbons-formation and deformation, as well as the fluorescenceluminescence behavior of the stacking ribbons.

Overall, this thesis demonstrates a bottom-up approach to build dynamic smart materials, from molecular engineering of azobenzene-based switches to their integration into different types of supramolecular materials including liquid crystals, host-guest complexes and colloidal inorganic nano-objects. The future work will keep on developing new engineering strategies and methods of molecular switches, as well as the study of mechanism of transducing molecular switching across multiple length scales. 


\section{Samenvatting}

Het koppelen van moleculaire foto-switches aan supramoleculaire systemen maakt het mogelijk om dynamische, slimme materialen met foto-gevoelige functies te ontwikkelen, onder de voorwaarde dat de energie van foto-illuminatie kan worden omgezet naar andere vormen en kan worden versterkt over meerdere lengteschalen. In dit proefschrift focussen we op foto-switches gebaseerd op azobenzeen als dynamische bouwstenen, vanwege de eenvoudige synthese en functionalisatie, de hoge extinctie-coëfficiënt en quantum yields en vanwege de veranderingen van de moleculaire geometrie en het dipoolmoment door het proces van foto-isomerisatie. Een belangrijke uitdaging blijft het gebrek aan stabiliteit van de cis-vorm, wat de toepassing beperkt door het verlies van de cis-vorm-informatie. Bovendien is UV-straling ongewenst in een biologische omgeving, vanwege mogelijke weefselschade. Daarom zijn we begonnen met onderzoek naar gefluorideerde azobenzenen en de toepassing daarvan in supramoleculaire materialen, om daar vervolgens kinetische controle en gecontroleerd switchen door middel van zichtbaar licht mogelijk mee te maken.

In Hoofdstuk 1 presenteren we een kort overzicht van veelgebruikte moleculaire fotoswitches.

In Hoofdstuk 2 bespreken we recente design- en engineeringstrategieën gericht op het ontwikkelen van azobenzenen die stabiel zijn in de cis-vorm. Deze strategieën omvatten ortho-fluoridering, ortho-methoxylering, azoheteroareen, hydrazon, azobenzeen- $\mathrm{BF}_{2}$ conjugatie en cyclisch azobenzeen.

In Hoofdstuk 3 worden twee strategieën, ortho-fluoridering en axiale chiraliteit, 
gecombineerd tot azobenzeen-gebaseerde, dynamische dotering voor vloeibare kristallen. In zowel de isotrope oplossing als in de vloeibaar kristallijne fase vertonen deze chirale doteermiddelen betere stabiliteit in de $c i s$-vorm dan hun niet-gefluorideerde tegenhangers. In de vloeibaar kristallijne fase kunnen stabiele helixstructuren met doteermiddelen in de cisvorm worden gevormd door middel van bestraling. Gefluorideerde doteermiddelen worden echter in verband gebracht met lichte verdraaiingen en variaties daarop van de helix, als gevolg van foto-isomerisatie in cholesterische vloeibare kristallen. Dit cholesterisch kristal kan potentieel worden toegepast in displays en foto-gevoelige actuators.

In Hoofstuk 4 onderzoeken we de host-guest-chemie van ortho-gefluorideerde azobenzenen in twee bekende host cavities, namelijk $\beta$-cyclodextrine en cucurbit[8]uril. In de aanwezigheid van beide host cavities vormen ortho-gefluorideerde azobenzenen stabiele supramoleculaire complexen. In de trans-vorm is de binding van $\beta$-cyclodextrine gunstiger, terwijl in de cis-vorm de binding van cucurbit[8]uril wordt bevoordeeld. Door lichtgeïnduceerde foto-isomerisatie tonen we aan dat de foto-switch op een reversibele manier kan worden overgebracht van de ene naar de andere host cavity.

In Hoofstuk 5 wordt de host-guest-chemie van ortho-gefluorideerde azobenzenen gebruikt voor het design van multivalente structuren, met als uiteindelijk doel om dynamische supramoleculaire nanodeeltjes te maken die zelforganiseren onder invloed van licht. In het tweecomponentensysteem, bestaande uit gefluorideerde azobenzenen en $\beta$ cyclodextrines, is de bindingsconstante in de zelfde orde van grootte voor de cis- en de transvorm, waardoor de supramoleculaire nanodeeltjes niet reageren op foto-isomerisatie. Vervolgens werd cucurbit[8]uril in het systeem geïntroduceerd om te binden aan cis- 
azobenzeen, in de verwachting dat de supramoleculaire nanodeeltjes uiteen zouden vallen als gevolg van competitieve complexatie. De binding tussen azobenzenen en $\beta$-cyclodextrines is echter versterkt door het effect van multivalentie, waardoor cucurbit[8]uril niet kan concurreren met $\beta$-cyclodextrine in de host-guest-complexatie, met als gevolg dat het supramoleculaire systeem ongevoelig bleef voor licht.

Tegenwoordig is een andere interessant vraagstuk de gecontroleerde dynamische zelfassemblage en dispersie door stimuli. In Hoofdstuk 6 worden foto-switches gebaseerd op azobenzeen toegepast als lichtgevoelige liganden voor nanoplaatjes van anorganische halfgeleiders. $\mathrm{Na}$ bestraling veroorzaakt de dipool-dipool-interactie face-to-face zelforganisatie van de nanoplaatjes en worden supra-specifieke linten gevormd. De volgende stap is het onderzoeken van het mechanisme achter de formatie en deformatie van deze linten, evenals de fluorescentie-luminescentie van gestapelde linten.

Kortom, dit proefschrift demonstreert de bottom-up aanpak om dynamische, slimme materialen te maken, van de moleculaire engineering van switches gebaseerd op azobenzeen tot de integratie daarvan in supramoleculaire materialen, zoals vloeibare kristallen, hostguest-complexen en colloïdale anorganische nanostructuren. Toekomstig onderzoek zal gewijd zijn aan het verder ontwikkelen van nieuwe engineeringstrategieën en methodes voor moleculaire switches, evenals het bestuderen van het mechanisme achter het omzetten van moleculaire switches op verschillende lengteschalen. 


\section{Acknowledgements}

To complete this dissertation, I would like to take this opportunity to thank everyone, who has supported me through my $\mathrm{PhD}$ period.

First of all, I would like to acknowledge my supervisor, Prof. Nathalie Katsonis for giving me the opportunity to do my $\mathrm{PhD}$ in your research group with atmosphere of great innovations, also for the guidance, especially the thinking of science behind the experimental details. More important, Nathalie, your attitude on scientific challenges always encourages me. If everything works in the first try, then it is so boring. It is a great pleasure for me to work with you. Besides, as your prophecy, I do drink coffee after dinner at 21.00 after 4 years PhD period.

Next, I would like to acknowledge to head of the BNT cluster, Prof. Jeroen Cornelissen, thank you for your help during my beginning and your hard work on running the cluster. You are always so energetic, which provides motivations to all the people around you.

Then I would like to sincerely thank my committee members: Prof. Jurriaan Huskens from University of Twente, Prof. Jennifer Herek from University of Twente, Prof. Wesley Browne from University of Groningen, Dr. Benoit Fleury from Sorbonne Université. Thank you for the kind suggestions and advices to my thesis and your participation in my defense ceremony.

I am also grateful to all BNT/MnF cluster members, Jurriaan, Tibor, Wim, Pascal, Melissa, Jos for your scientific advices and discussions during colloquium, meetings and 
also in the coffee corner. Every single discussion brings me fresh air to my mind. Jurriaan, I really enjoy the way you present your scientific opinion, you could always get on the point with most simple and direct words. Tibor, a discussion with you is always fruitful, I would also like to say congratulations to you for your new coming child. Special thanks to our laboratory supporting team, technicians Marcel, Regine, Bianca, Richard and secretaries Nicole and Izabel. It is not easy for me as a foreign student to work and live in a new environment, thank you so much for helping me both inside and outside the lab.

Moreover, I would like to acknowledge all my collaborators, Alberto, Yessica Flores, Dr. Benoit Fleury, and Dr. Rico Keim for TEM measurements, Mark Smithers for SEM measurements. It is my pleasure to have the opportunity to work with you together.

I would like to acknowledge my lovely group members and colleagues who helped me to finalize the thesis and go through this 4 years together. Remi, thank you for checking this long, long and boring experimental details word by word, Jonathan, thank you for translating the summary to dutch. Federico and Alexander, thank you for being my paranyphms and helping me to organize all the things for my defense, you guys are really talent, you will definitely make great success on science (and nature and nature chem and nature mater, etc. of course). Ben, Sara, Supitch, Piotr, Rindia and Rianne, I am so pleased to meet you in the beginning of my $\mathrm{PhD}$, your warm welcoming helped me a lot to start to work in a new environment smoothly. Supa, you are much more tough and strong than you look like, I think I can already say congratulations to you for your PhD. Wilfred and Pichayut, bad and worse singer, thank you for constructing such a warm atmosphere in the lab. Also grateful to Goodday, Alexis, Tetiana, Dhanya, I enjoy the time that working with 
you together.

Meeting the people from home in distant land is one of the best happiness. Liulin and Wei, there is no doubt that you will be great scientist. Aijie, we start the $\mathrm{PhD}$ almost together, your fortitude and self-determination encourage me all the time like a mirror. Shuqin, Liang, Fangyuan, Yao, Kun, Qin, Liuyi, Min, it is my pleasure to meet you here, $8000 \mathrm{~km}$ away from home, wish you all the best in the future.

I also want to say thank you to all the colleague from the BNT/MnF cluster. Andrea, as you were there every weekend, I am not alone in the lab, which makes me feel much better. L'Ale, good luck my gym mate. Laura, thank you so much for helping me move twice. Maike Wiemann, it is a nice experience to me to have you as a desk mate, herzlich Glückwunsch und alles Gute für die Zukunft. Raquel, do you know how important you are, as a warm, kind organizer for all kind of party, dinner and group activities in such a big working cluster! To the boys from BNT/MnF futsal team: Jacopo, Andy, Mark Verheijden, Roberto, Rick, Joao, Csaba, Ivan, Lorenzo, Jonathan, Albert, Francesco, go ahead and keep on winning guys! And Anne, Martijn, Rik, Stan, Mark de Ruiter, Stan, Robin, Pia, Disraeli, Gaurav, Maike Brand, Victoria, Naomi, Melissa, Almudena, Nicolas, Daniele, Erik, Gulistan, Sara, Alexander Milbrat, Nico, Pramod, Luca, Wouter, Nicole, Emanuela, Jenny, Sven, Rajesh, Shrikrishnan, Tushar, Carmen, Tom, Betty, Jasper, Wies, Jens, I wish I did not miss any name here, it is my great pleasure to meet you here and working together.

I would also like to acknowledge the Chinese student association in Enschede, and all the friends I meet here, Lantian, Ying, Jinfeng, Haishan, Shanqiu, Yunlong, Xueling, 
Kaihuan, Minmin, Xiaohua, Kenan, Youwen, Mengdi, Rong, Lijie, Peng, Lei, Wanshu, Yao, Jiahui, Junwen, with my best wishes. A special place for our football team CN Old Boys, Zhou Lu, Chao Wang, Yin Tao, Jin Cui, Hang Song, Xiang Zhang, Yi Wang, Xingwu Sun, Xichen Zhang, Yue Ma, Ziwei Li, Yi Li, Yulai Wang, Amy Jia, we made a great historical progress last year, forward Old Boys!

The final part is for my family. 感谢我的父母, 无论何时, 无论何地你们永远都在我 的背后支持我。感谢我的妻子吴立群, 感谢你的陪伴, 以后的日子让我们携手一起面 对。

Enschede, March 2018

He Huang 


\section{About the author}

He Huang was born on $20^{\text {th }}$ of April 1987 in Liaoning, China. He pursued his bachelor degree in chemistry, in department of chemistry at Fudan University, Shanghai, China, and graduated in July 2009. During his bachelor thesis, he studied new synthesis route of Lcarnitine under the supervision of Prof. Fener Chen. From October 2010 to August 2013, he pursued his master degree in chemistry, in faculty for mathematic, informatic and nature science, RWTH Aachen University, Germany. His master thesis, entitled "Surface segregation of amphiphilic branched polyglycidols additive in poly (vinylidene fluoride) host" was carried out in the DWI institute, RWTH Aachen university under the supervision of Prof. Martin Möller.

Since $15^{\text {th }}$ of November 2013, he started his PhD program in the Bio-Inspired and Smart Material group under the supervision of Prof. Nathalie Katsonis. The aim of his project is to study the molecular switch azobenzenes as energy transducers in dynamic supramolecular systems. The results of this project are described in this thesis. 


\section{List of publications}

1. H. Huang, A. Juan, N. Katsonis, J. Huskens, Competitive inclusion of molecular photoswitches in host cavities. Tetrahedron, 2017, 73, 4913-4917.

2. H. Huang*, T. Orlova*, B. Matt, N. Katsonis, Long-lived Supramolecular Helices Promoted by Fluorinated Photoswitches, Macromol. Rapid Commun., 2017, 1700387. (* the authors contributed equally to the work)

3. Y. Flores*, H. Huang*, R. Plamont*, B. Fleury, N. Katsonis, Photo-responsive SelfAssembly of Anisotropic Hybrid Nano-Objects. Manuscript in preparation (* the authors contributed equally to the work).

4. H. Huang, N. Katsonis, Bi-stable azobenzenes as energy tranducers in supramolecular systems. Manuscript in preparation.

\section{Other publications}

5. D. Enders, A. Grossmann, H. Huang, G. Raabe, Dual Secondary Amine/N-Heterocyclic Carbene Catalysis in the Asymmetric Michael/Cross-Benzoin Cascade Reaction of $\beta$-Oxo Sulfones with Enals. Eur. J. Org. Chem., 2011, 23, 4298-4301.

6. H-J. Zhang, P. Becker, H. Huang, R. Pirwedjan, F-F. Pan, C. Bolm, Photochemically Induced Silyacylations of Alkynes with Acylsilanes, Adv. Syn. Cat., 2012, 354, 2157-2161.

7. L. Wang, H. Huang, D. L. Priebbenow, F-F. Pan, C. Bolm, Copper-Catalyzed Oxidative Cross-Coupling of Sulfoximines and Alkynes. Angew. Chem. Int. Ed., 2013, 52, 3478-3480. 